\title{
₹USGS

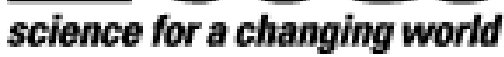

\section{Calculating depths to shallow magnetic sources using aeromagnetic data from the Tucson basin}

By Daniel W. Casto ${ }^{1}$

Open-File Report 01-505

2001

This report is preliminary and has not been reviewed for conformity with U.S. Geological Survey editorial standards or with the North American Stratigraphic Code. Any use of trade, product or firm names is for descriptive purposes only and does not imply endorsement by the U.S. Government.

\section{U. S. DEPARTMENT OF THE INTERIOR \\ U.S. GEOLOGICAL SURVEY}

1U.S. Geological Survey, Tucson, Arizona. This Master's Thesis was funded by the Southwest Mineral and Environmental Investigations Project, Mineral Resources Program, Geologic Division, U.S. Geological Survey. 
CALCULATING DEPTHS TO SHALLOW MAGNETIC SOURCES

USING AEROMAGNETIC DATA

FROM THE TUCSON BASIN

By

Daniel W. Casto

\author{
A Thesis Submitted to the Faculty of the \\ DEPARTMENT OF MINING AND GEOLOGICAL ENGINEERING \\ In Partial Fulfillment of the Requirements \\ For the Degree of \\ MASTER OF SCIENCE \\ WITH A MAJOR IN \\ MINING, GEOLOGICAL \& GEOPHYSICAL ENGINEERING \\ In the Graduate College \\ THE UNIVERSITY OF ARIZONA
}




\section{STATEMENT BY AUTHOR}

This thesis has been submitted in partial fulfillment of requirements for advanced degree at the University of Arizona and is deposited in the University Library to be made available to borrowers under the rules of the Library.

Brief quotations from this thesis are allowable without special permission, provided that accurate acknowledgement of source is made. Requests for permission for extended quotation from or reproduction of this manuscript in whole or in part may be granted by the head of the major department or the Dean of the Graduate College when in his or her judgement the proposed use of the material is in the interests of scholarship. In all other instances, however, permission must be obtained by the author.

SIGNED:

APPROVAL BY THE THESIS DIRECTOR

The thesis has been approved on the date shown below:

Mark E. Gettings

Date

Adjunct Professor, Geophysics

Ben K. Sternberg

Date

Professor, Geophysical Engineering 


\section{ACKNOWLEDGEMENTS}

There are several people to whom I owe much gratitude for making the last two years of my life an enjoyable and rewarding experience. First and foremost, I would like to thank Mark Gettings for his limitless kindness and support. Without him, I would never have gotten to the place that I am now, in both mind and spirit. I would also like to thank Mark Bultman and everyone else at the U.S. Geological Survey for their willingness to help whenever I needed it. I have great appreciation for the financial support of the U.S.G.S., which made these past two years possible. I would like to thank my advisor, Ben Sternberg, and the rest of the faculty and staff of the department for their helpfulness and encouragement these past two years. I'd also like to thank my family and friends for driving me to succeed. Last, but certainly not least, I would like to thank Phil Hammonds for his incredible patience and assistance. Without his troubleshooting, there is no doubt in my mind that I would be working on this thesis for another 10 years! 


\section{TABLE OF CONTENTS}

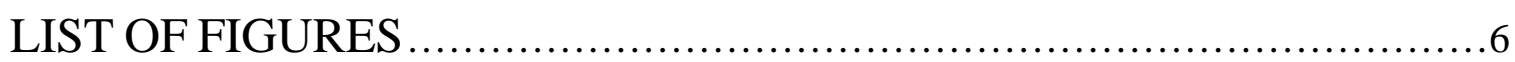

LIST OF TABLES ............................................................

ABSTRACT .............................................................................

INTRODUCTION ........................................................... 12

GEOLOGIC SETTING ...........................................................

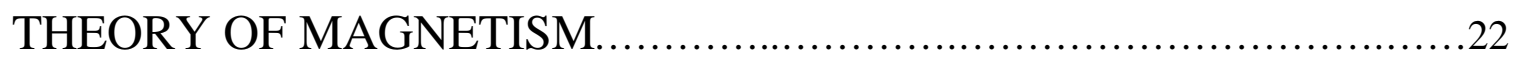

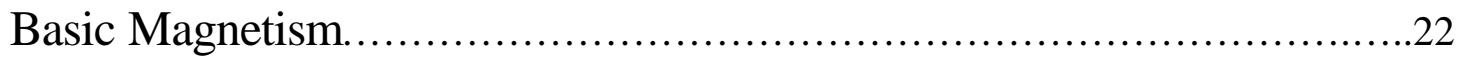

The Geomagnetic Field ..................................................27

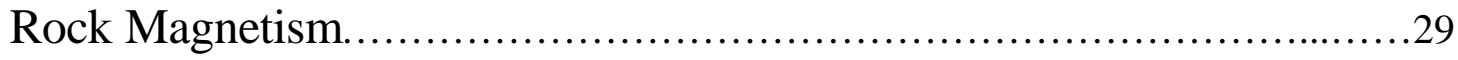

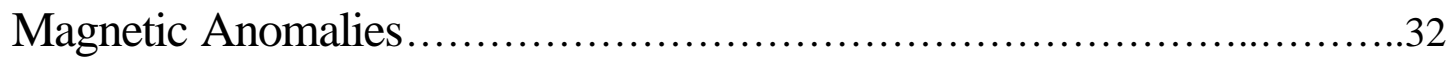

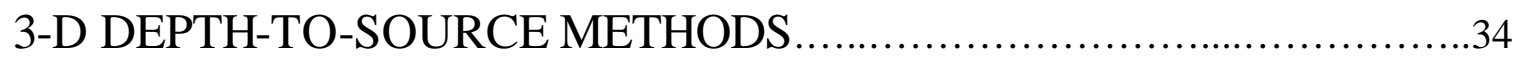

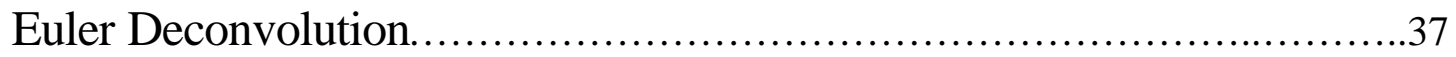

Simple Analytic Signal...................................................41

Horizontal Gradient.....................................................47

Enhanced Analytic Signal.....................................................50

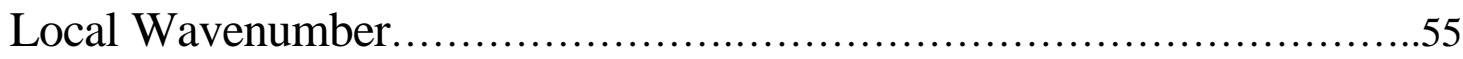

DATA ACQUISITION AND PROCESSING .................................59

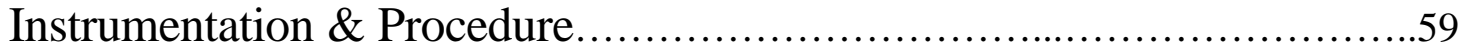

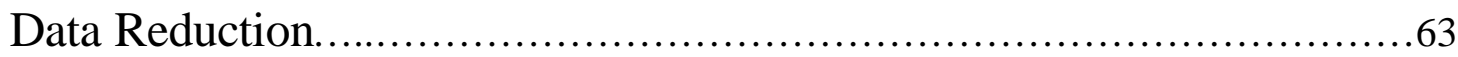


TABLE OF CONTENTS - continued

Derivative \& Depth Calculations .................................................66

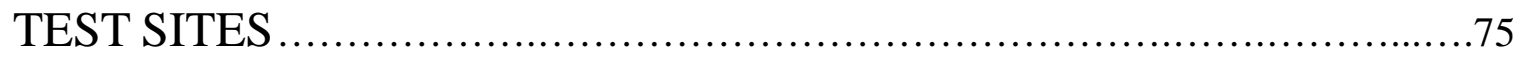

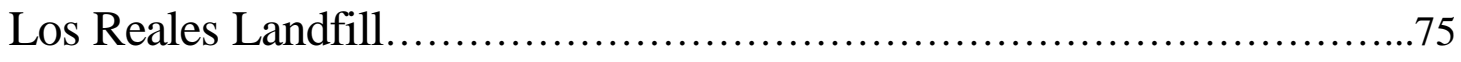

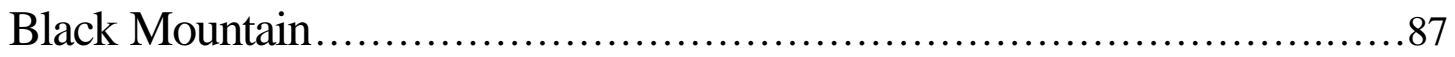

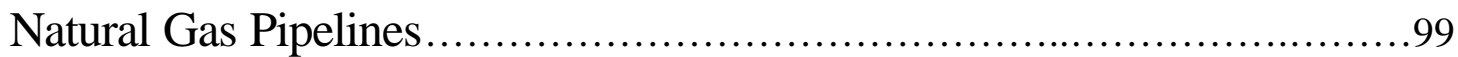

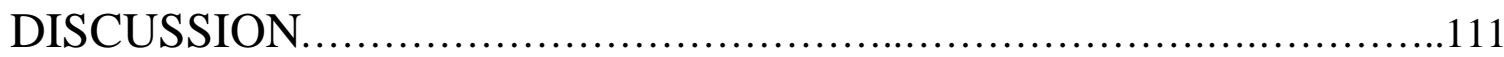

Gridding and Filtering ..................................................... 111

Best and Worst Methods...................................................123

GEOLOGIC SITES OF INTEREST ........................................131

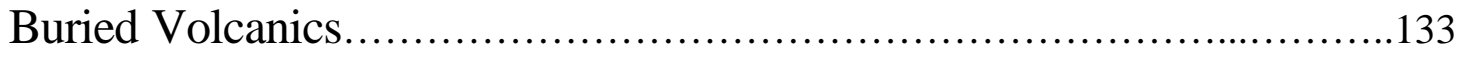

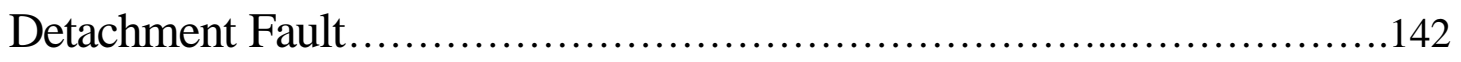

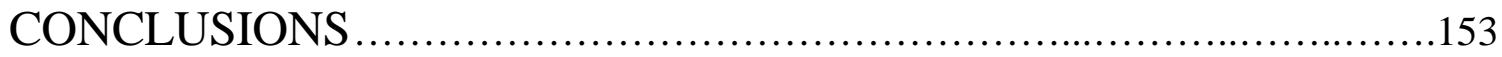

Depth Calculation Guidelines..............................................153

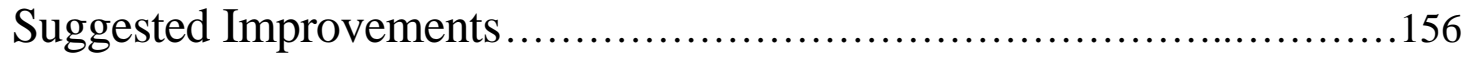

APPENDIX A: Depth-to-source maps over the Los Reales Landfill.........158

APPENDIX B: Depth-to-source maps over Black Mountain..................181

APPENDIX C: Depth-to-source maps over the Natural gas pipelines.......206

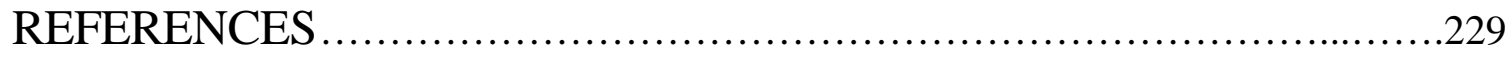




\section{LIST OF FIGURES}

Figure 1 Map of the Tucson basin within the Basin and Range of southern Arizona, along with the location of the Exxon State (32)-1 well.....................15

Figure 2 Stratigraphic column of rocks in the Exxon State (32)-1 well................17

Figure 3 Vector representation of the geomagnetic field with and without a superimposed magnetic anomaly.....................................33

Figure 4 The two-dimensional simple analytic signal amplitude over a magnetic contact..........................................................44

Figure 5 The two-dimensional horizontal gradient magnitude over a magnetic contact..............................................................

Figure 6 Plot of ratio of thickness $t$ to depth $d$ below the observation level versus estimation error of depth $d$ due to a vertical step model enhanced analytic signal approach.........................................................

Figure 7 The two-dimensional local wavenumber over a magnetic contact.............56

Figure 8 Aerial extent of the aeromagnetic survey conducted over the Tucson basin by Sander Geophysics, Ltd................................................60

Figure 9 Total-field magnetic anomaly map of the entire survey area.................68

Figure 10 Location of the Los Reales Landfill in southeastern Tucson...................76

Figure 11 Topographic map created from radar altimeter and elevation data collected during the survey over the Los Reales Landfill................................77

Figure 12 Total magnetic field anomaly map over the Los Reales Landfill...............78

Figure 13 Depth-to-source error histograms for each method over the Los Reales Landfill.

Figure 14 Location of Black Mountain southwest of Tucson...........................88

Figure 15 Topographic map created from radar altimeter and elevation data collected during the survey over Black Mountain. 


\section{LIST OF FIGURES - continued}

Figure 16 Total magnetic field anomaly map over Black Mountain..................90

Figure 17 Depth-to-source error histograms for each method over Black Mountain....95

Figure 18 Location of the natural gas pipelines southeast of Tucson.................100

Figure 19 Topographic map created from radar altimeter and elevation data collected during the survey over the natural gas pipelines........................101

Figure 20 Total magnetic field anomaly map over the natural gas pipelines............102

Figure 21 Depth-to-source error histograms for each method over the natural gas pipelines

Figure 22 The simple and second-order analytic signal amplitudes over the Los Reales Landfill calculated from a 5-meter grid

Figure 23 The simple and second-order analytic signal amplitudes over the Los Reales Landfill calculated from a low-pass filtered 5-meter grid.................114

Figure 24 The total magnetic field anomaly due to the landfill at the flight elevation and at an upward continuation of 150 meters..............................116

Figure 25 Comparison of the number of depth estimates using the simple analytic signal method over the landfill at the flight elevation and at an upward continuation of 150 meters.

Figure 26 The second-order analytic signal amplitude over the landfill that was calculated with a 50-meter grid spacing and calculated with a 5-meter grid spacing, then re-gridded at 50 meters.

Figure 27 Comparison of the number of depth estimates using the simple analytic signal method over the landfill with different search window sizes....

Figure 28 Profile of the second order analytic signal amplitude over the Los Reales

Landfill before and after applying a low-pass filter.

Figure 29 Median depth error for each method over each target...................125

Figure 30 Depth-to-source method ratings...................................126 


\section{LIST OF FIGURES - continued}

Figure 31 Cross-section of the magnetic field due to a pipeline with cathodic

protection.

Figure 32 Location of the Mission Mine area....

Figure 33 High-pass filtered magnetic field anomaly map showing a reversed anomaly beneath the Los Reales Landfill.......................................134

Figure 34 Map showing reversed anomalies just west of Tucson...................135

Figure 35 Map of the simple analytic signal amplitude over the suspected buried volcanics

Figure 36 Depth-to-source histograms for each method over the suspected buried volcanics

Figure 37 Depth-to-source map over the suspected buried volcanics using the local wavenumber method applied to the vertical integral of the total magnetic field anomaly....

Figure 38 Depth-to-source map over the suspected buried volcanics using the horizontal gradient method applied to the pseudo-gravity anomaly field.

Figure 39 Total-field magnetic anomaly map showing the apparent offset of similar magnetic anomalies east of the Twin Buttes mine area.

Figure 40 Depth-to-source histograms for each method over the detachment fault.

Figure 41 Depth-to-source map over the detachment fault using the simple analytic signal method.

Figure 42 Depth-to-source map over the detachment fault using the local wavenumber method applied to the vertical integral of the total magnetic field anomaly....

Figure 43 Depth-to-source map over the detachment fault using the horizontal gradient

method applied to the pseudo-gravity anomaly field... 


\section{LIST OF FIGURES - continued}

Figure 44 Depth-to-source map over the detachment fault using the horizontal gradient method applied to the reduced-to-pole total magnetic field anomaly....

Figure 45 Shape of the two-dimensional horizontal gradient magnitude of the pseudogravity field over a contact when the source magnetization is not vertical 


\section{LIST OF TABLES}

Table 1 Depth-to-source error statistics for the Los Reales Landfill...................80

Table 2 Depth-to-source error statistics for Black Mountain........................92

Table 3 Depth-to-source error statistics for the natural gas pipelines................104

Table 4 Depth-to source statistics for the suspected buried volcanics...............137

Table 5 Depth-to-source statistics for the detachment fault.......................144 


\begin{abstract}
Using gridded high-resolution aeromagnetic data, the performance of several automated 3-D depth-to-source methods was evaluated over shallow control sources based on how close their depth estimates came to the actual depths to the tops of the sources. For all three control sources, only the simple analytic signal method, the local wavenumber method applied to the vertical integral of the magnetic field, and the horizontal gradient method applied to the pseudo-gravity field provided median depth estimates that were close $(-11 \%$ to $+14 \%$ error) to the actual depths. Careful attention to data processing was required in order to calculate a sufficient number of depth estimates and to reduce the occurrence of false depth estimates. For example, to eliminate sampling bias, high-frequency noise and interference from deeper sources, it was necessary to filter the data before calculating derivative grids and subsequent depth estimates. To obtain smooth spatial derivative grids using finite differences, the data had to be gridded at intervals less than one percent of the anomaly wavelength. Before finding peak values in the derived signal grids, it was necessary to remove calculation noise by applying a low-pass filter in the grid-line directions and to re-grid at an interval that enabled the search window to encompass only the peaks of interest. Using the methods that worked best over the control sources, depth estimates over geologic sites of interest suggested the possible occurrence of volcanics nearly 170 meters beneath a city landfill. Also, a throw of around 2 kilometers was determined for a detachment fault that has a displacement of roughly 6 kilometers.
\end{abstract}




\section{INTRODUCTION}

The purpose of this study was to gain a better understanding of how to calculate and interpret three-dimensional depth-to-source estimates using automated techniques on gridded potential-field data. One of the most useful applications of magnetic data has been to determine the depth to the top of magnetic sources. In hydrocarbon exploration, this type of application gives the thickness of the sedimentary section. In mineral exploration, depth estimates are important in determining the depth of ore bodies that contain magnetic minerals (Thompson, 1982). On a smaller scale, depth estimates to cultural sources may be important to environmental remediation efforts. Because of the usefulness of this application of magnetic data, there has been a continuing effort to develop improved techniques that yield accurate depth-to-source estimates (Thompson, 1982).

Recently, there has been an increase in use of automated interpretation techniques, as faster computers and commercial software are now widely available (Reid et al., 1990). Many of these automated techniques are capable of rapidly calculating depth estimates from large grids of data. However, their reliability is questionable due to a lack of adequate testing. Previous studies have only tested these techniques on a few deep, thick, large-scale geologic targets. In this study, several automated interpretation techniques were tested using relatively shallow, thin sources whose geometry and depth were precisely known. 
Currently, there is no single correct method of calculating depth-to-source estimates using potential-field data. However, there are several incorrect ways of approaching the problem. This project's aim was to provide a set of gidelines to aid in the calculation and interpretation of three-dimensional depth-to-source estimates and to prevent the occurrence of false depth estimates. These guidelines were derived from test calculations made with high-resolution aeromagnetic data over different control sources within the Tucson basin using several automated techniques. 


\section{GEOLOGIC SETTING}

The Tucson basin is a nearly 2,600 square-kilometer area in Pima County, southeastern Arizona. It is a sediment-filled structural depression that trends north to northwest and lies within the Basin and Range physiographic province (Figure 1) (Anderson, 1987). The basin is bounded by rugged mountains that rise abruptly above a broad and gently sloping valley floor. The mountains that surround the basin, which include the Tucson, Black, Sierrita, Santa Rita, Empire, Rincon, Tanque Verde, Santa Catalina and Tortolita Mountains, are composed of igneous, metamorphic and sedimentary rocks that range in age from Precambrian to Tertiary (Anderson, 1987).

The present-day Tucson basin is the result of a variety of geologic processes that occurred during the Cenozoic era. Many of the oldest deposits that fill the Tucson basin accumulated during a detachment-related structural regime (Anderson, 1987). Subsequent block faulting and sedimentation resulted in the formation and eventual burial of a deep structural trough trending north to northwest along the central axis of the modern valley floor. Continued erosion, pedimentation and deposition of the sediment following the cessation of block faulting enlarged the Tucson basin to its present-day extent (Anderson, 1987).

In 1972, Exxon Company, U.S.A. drilled a 3,828 meter-deep (12,556 ft) test well near the center of the Tucson basin (Exxon State (32)-1, Sec. 5, T. 16 S., R. 15 E., Pima County, Arizona) (Figure 1). The well was drilled near the deep part of the basin that provided a thick, relatively complete sedimentary section with few hiatuses or 


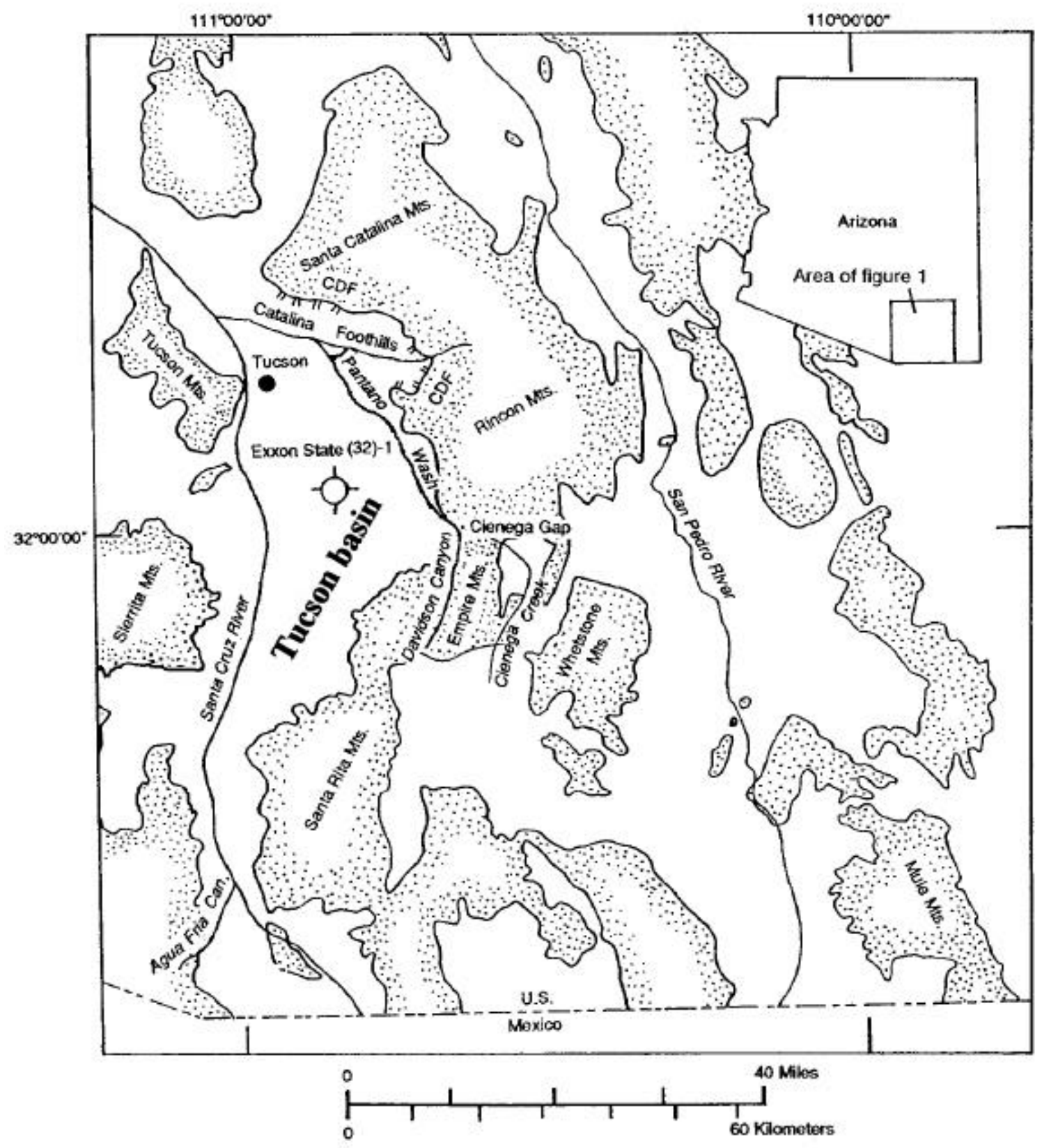

Figure 1. Map of the Tucson basin within the Basin and Range of southern Arizona, along with the location of the Exxon State (32)-1 well (modified from Houser and Gettings, 2000). 
complicating influxes of locally derived sediment (Houser and Gettings, 2000). A detailed study of cuttings and geophysical logs from the Exxon State (32)-1 exploration well, in addition to surface data in the area and other deep wells in the basins of southwestern Arizona, has led to a revised subsurface stratigraphy for the basin from that reported by Davidson (1973) and Anderson (1987) and provided new insight into the tectonic history of the basin. The well has provided the most comprehensive record of the stratigraphy of the Tucson basin available today (Houser and Gettings, 2000).

The stratigraphic section that was identified in the Exxon well is as follows: Pleistocene(?) to upper Miocene upper basin-fill sedimentary rocks (0-909 m); upper and middle Miocene lower basin-fill sedimentary rocks (909-1,881 m); lower Miocene and upper Oligocene(?) Pantano Formation (1,881-2,517 m); lower Miocene(?) and Oligocene middle Tertiary volcanic and sedimentary rocks $(2,517-3,057 \mathrm{~m})$; Lower Cretaceous to Upper Jurassic Bisbee Group (3,057-3,659 m); pre-Upper Jurassic granitoid crystalline rock (3,659-3,828 m TD) (Figure 2) (Houser and Gettings, 2000).

The upper 1,881 meters of sediments in the Tucson basin have been informally broken up into four units by Houser and Gettings (2000), Units A and B of the upper basin fill and Units $\mathrm{C}$ and $\mathrm{D}$ of the lower basin fill (Figure 2). The upper and lower basin-fill sediments have been separated based on age, degree of consolidation and amount of deformation. The upper basin fill is generally Pliocene and Pleistocene in age, poorly to moderately consolidated, flat lying and broken by only a few small displacement faults. The lower basin fill is generally middle to late Miocene in age, moderately consolidated, slightly to moderately deformed with dips of as much as $15^{\circ}$ 


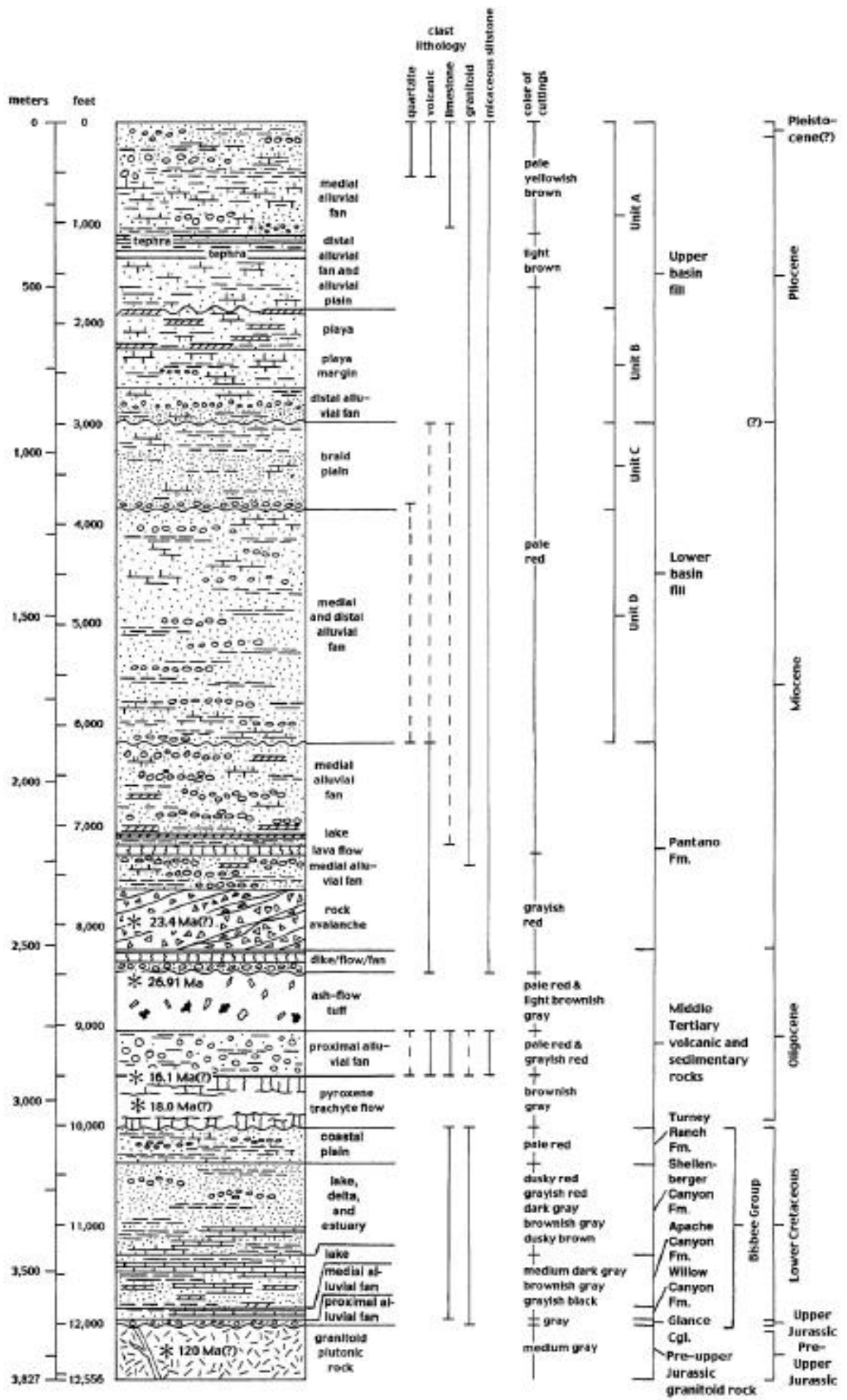

Figure 2. Stratigraphic column of rocks in the Exxon State (32)-1 well. Dashed lines under clast-lithology heading indicate intervals where the lithology is rare (modified from Houser and Gettings, 2000). 
and broken by numerous faults ranging in displacement from small to very large (Houser and Gettings, 2000).

The areal distribution of the upper and lower basin-fill facies indicates they were deposited in basins having a somewhat modern configuration. The upper basin fill was derived from adjacent ranges and consists mainly of alluvial fan, alluvial plain and playa deposits. The lower basin fill does not contain lithologies that are present or locally abundant in the adjacent ranges, which has been attributed to erosional stripping, but also consists mainly of fluvial deposits (Houser and Gettings, 2000).

Just below the upper and lower basin-fill sediments is the Pantano Formation (Figure 2). This formation was encountered between 1,881 and $2,517 \mathrm{~m}$ in the Exxon State (32)-1 well and can be seen in outcrop approximately 7 to 9 kilometers east of the well site. Its age has been approximated to be lower Miocene and upper Oligocene. The Pantano Formation is made up of syntectonic deposits of alluvial fans, rock avalanches and volcanic flows in a region of middle Tertiary extension. In the Exxon State (32)-1 well, two parts to the Pantano Formation were encountered. The top $449 \mathrm{~m}$ of the Pantano Formation consists of well-consolidated gypsiferous, muddy conglomerate containing an andesite flow in the lower half. Beneath this is a 187 mthick sequence of rock-avalanche deposits that are comprised mostly of intermediate-composition volcanic rocks (Houser and Gettings, 2000).

Beneath the Pantano Formation lie middle Tertiary volcanic and sedimentary rocks penetrated from 2,517 to 3,057 $\mathrm{m}$ in the Exxon State (32)-1 well (Figure 2). These rocks have been estimated to be lower Miocene and Oligocene in age. The $540 \mathrm{~m}$ thick 
interval consists of a diverse group of rocks with uncertain correlation to surface units. It contains six different identifiable rock types: a lamprophyre(?) dike, an intermediatecomposition lava flow, a conglomerate, a crystal-lithic ash-flow tuff, a limestone conglomerate and a pyroxene trachyte flow (Houser and Gettings, 2000).

Just beneath the middle Tertiary rocks are the lower Cretaceous and Upper Jurassic rocks belonging to the Bisbee Group (Figure 2). These rocks were encountered between depths of 3,057 and 3,659 $\mathrm{m}$ in the Exxon State (32)-1 well and consist mainly of shale, thin-bedded limestone and conglomerates. In the Empire and Whetstone Mountains, 9 to 18 kilometers southeast of the well site, the Bisbee Group is comprised of five formations: the basal Glance Conglomerate and overlying Willow Canyon Formation, Apache Canyon Formation, Shellenberger Canyon Formation and Turney Ranch Formation. All but the Willow Canyon Formation were found in the Exxon State (32)-1 well, however they were much thinner than those found in the Empire and Whetstone Mountains.

The Bisbee Group was deposited in the Bisbee basin, which pre-dates the presentday Tucson basin. The Bisbee basin formed as a series of block-faulted, asymmetrical, northwest-trending, en-echelon extensional sub-basins in response to the rifting that created the Chihuahua trough and formed the Gulf of Mexico (Houser and Gettings, 2000). Two explanations for the thinner formations and missing Willow Canyon Formation of the Bisbee Group discovered at the well site have been postulated. Either the well was located close to the northwestern extent of rifting that formed the Bisbee 
basin (and hence at a shallower portion of the basin), or the well was located at the shallower end of a half-graben Bisbee sub-basin (Houser and Gettings, 2000).

At 3,659 $\mathrm{m}$ in the Exxon State (32)-1 well, an equigranular granitoid crystalline rock was encountered (Figure 2). The well penetrated $169 \mathrm{~m}$ of this rock, and drilling stopped at $3,828 \mathrm{~m}$. The age of the granitoid body can only be estimated at preCretaceous. The granitoid chips from cuttings are composed of quartz and feldspar along with muscovite, biotite or chlorite, with mafic minerals being rare. There is evidence from geophysical logs, which include density, gamma, sonic and resistivity, that the granitoid body is cut by several dikes, and that there may be two different igneous bodies separated by a fault (Houser and Gettings, 2000).

The geologic events that affected the Tucson basin in the vicinity of the Exxon State (32)-1 well site were summarized by Houser and Gettings (2000) based on their interpretation of the well data and surrounding exposures. They suggested the following events, in the order from oldest to youngest: emplacement of the granitoid crystalline rock, probably in Middle Proterozoic time (1,400 Ma); probable deposition and subsequent erosion of Paleozoic marine sedimentary rocks; northeast-southwest directed extension, beginning in latest Jurassic time and continuing through the Early Cretaceous, creating the Bisbee basin in which fluvial, lacustrine and marine sedimentary rocks of the Bisbee Group were deposited; late Cretaceous to middle Tertiary hiatus; magmatism and northeast-southwest directed crustal extension during the middle Tertiary (mid-Tertiary orogeny); detachment faulting during the middle Tertiary that caused the deposition of the Pantano Formation (and, according to Anderson (1987), deformation and uplift of the 
metamorphic core complex rocks that make up the Tortolita, Santa Catalina, Tanque Verde and Rincon Mountains); an early Miocene to middle Miocene hiatus; and finally basin and range extension and faulting, responsible for the basin-fill sedimentary rocks in the uppermost 1,881 meters. 


\section{THEORY OF MAGNETISM}

\section{Basic Magnetism}

The purpose of magnetic surveying is to investigate the subsurface based on variations in the Earth's magnetic field that result from the magnetic properties of the underlying rocks, or in some cases cultural sources. In order to understand these variations, it is important to first understand the basic concepts of magnetism and how subsurface geology can affect the measured Earth's magnetic field. The following discussions are based on material from Kearey and Brooks (1991) and Telford et al. (1990), unless otherwise noted.

In classical magnetic theory, the mutual attraction (or repulsion) of two point magnetic poles is considered. Magnetic poles can interact with one another at distance by means of their magnetic fields. The force $F$ between two magnetic poles of strength $m_{1}$ and $m_{2}$ separated by a distance $r$ is given by

$$
F=\frac{\grave{i}_{0} m_{1} m_{2}}{4 \partial \grave{i}_{R} r^{2}}
$$

where $\mu_{\mathrm{o}}$ and $\mu_{\mathrm{R}}$ are constants corresponding to the magnetic permeability of vacuum and the magnetic permeability of the medium separating the poles, respectively. The force is attractive if the poles are of opposite sign and repulsive if they are of like sign. The sign convention is that a positive pole is attracted towards the Earth's north pole.

In order to measure the force due to a magnetic field in magnetic surveying, a more practical form of $F$ is needed. The magnetic field strength, $H$, is defined as the 
force exerted on a unit positive pole due to a pole of strength $m$ at a distance $r$ from the pole and can be expressed mathematically as

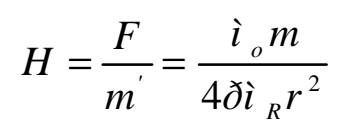

where $m^{\prime}$ is the unit positive pole and essentially the instrument used in measurement.

Common magnets, such as bar magnets, have a pair of poles and are referred to as magnetic dipoles. Magnetic dipoles have magnetic moments associated with them. The magnetic moment $M$ of a dipole with poles of strength $m$ and a distance $l$ apart is defined by

$$
M=m l .
$$

According to modern magnetic theory, all magnetic poles exist in pairs, or dipoles, in nature. Also, all magnetic fields are a direct result of the flow of electric current. A current flowing in a circular loop acts as a magnetic dipole located at the center of the loop. The magnetic field is oriented in the direction in which a right-handed screw would advance if turned in the direction of current flow. Its dipole moment $M$ is given by

$$
M=i A
$$

where $i$ is the current and $A$ is the cross-sectional area of the loop. This relationship between electric current and magnetism can also be seen at the atomic level.

On an atomic scale, all substances are magnetic. Both the spin of an atom's electrons and the orbital motions of the electrons around the nucleus constitute circular currents and cause atoms to behave as dipoles. These atomic dipoles also have magnetic 
moments associated with them. The magnetic dipole moments associated with electron motion are measured in terms of the Bohr magneton $\mu_{B}$, where

$$
\mu_{B}=\frac{e h}{4 \pi m}
$$

with $e$ being the elementary charge, $m$ the electron mass and $h$ being Planck's constant (Halliday et al., 1993). The dipole moment associated with intrinsic electron spin is almost exactly $-1 \mu_{B}$. The dipole moment associated with electron orbital motion $\mu_{o r b}$ is

$$
\mu_{o r b}=-\frac{e}{2 m} L_{o r b}
$$

where $L_{\text {orb }}$ is the orbital angular momentum (Halliday et al., 1993). Molecules also have spin, which gives them magnetic moments as well.

When a material is placed in a weak external magnetic field, it will acquire a magnetization in the direction of the field, which is lost when the material is removed from the field. This is referred to as induced magnetization and is caused by the reorientation of atoms within the material so that their electron spins line up with the direction of the applied field. The intensity of induced magnetization $J_{i}$ of a material is defined as the dipole moment per unit volume of material:

$$
J_{i}=\frac{M}{V}
$$

where $M$ is the magnetic moment and $V$ is the unit volume of the material.

The degree to which a material is magnetized is determined by that material's magnetic susceptibility. The susceptibility $k$ of a material is defined as the ratio of 
intensity of magnetization $J_{i}$ to the magnetizing field $H$ and is with respect to unit volume:

$$
k=\frac{J_{i}}{H}
$$

Magnetic susceptibility is the most important physical property measured in magnetics. Susceptibility is dimensionless, however in the SI system its value is greater than that in the c.g.s system by a factor of $4 \pi$.

The arrangement of atomic dipoles within a material defines that material's magnetic behavior. All materials can be classified as either diamagnetic or paramagnetic. In diamagnetic materials, all electron shells around the nuclei are filled and no unpaired electrons exist. Since quantum theory only allows a maximum of two electrons to exist in the same state provided they have opposite spins, the spin magnetic moments in diamagnetic materials cancel. When placed in an external magnetic field, the paths of the orbiting electrons in a diamagnetic material rotate to create a magnetic field in opposition to the applied field. This results in a very weak and negative susceptibility. In paramagnetic materials, the electron shells are incomplete, and the spin magnetic moments do not cancel. The unpaired electrons' spins rotate to produce a field in the same direction as the applied field. This results in a positive susceptibility, but is also very weak.

In some paramagnetic materials possessing several unpaired electrons, the dipoles associated with the spins of these electrons are magnetically coupled between adjacent atoms. The coupled magnetic moments constitute a single magnetic domain. The 
magnetic coupling can be either parallel or anti-parallel, depending on the degree of overlap among electron orbits. If the coupling is parallel, the result is a very strong magnetization and a high magnetic susceptibility. These materials are referred to as ferromagnetic and rarely occur naturally in the Earth's crust. Examples of ferromagnetic materials include iron, cobalt and nickel. If the coupling is anti-parallel, there are an equal number of dipoles in each direction and the magnetic effects are self-canceling. These materials are referred to as anti-ferromagnetic. An example of a naturally occurring anti-ferromagnetic material is hematite. In some materials, such as magnetite, the dipole coupling is similarly anti-parallel. However, the number of dipoles in each direction is unequal, giving rise to a net magnetization and a relatively high magnetic susceptibility. These materials are referred to as ferrimagnetic and make up nearly all of the minerals responsible for magnetic properties of common rock types.

The strength of magnetization of ferromagnetic and ferrimagnetic materials decreases as temperature increases and eventually disappears at and above the Curie temperature. The Curie temperature is the point at which interatomic distances are too great to allow for electron coupling between atoms. Above this emperature, the material behaves as an ordinary paramagnetic material. However, if a material is cooled through and below the Curie temperature in the presence of a magnetic field, it will inherit a permanent magnetization in the direction of that field. This permanent magnetization is often referred to as remanent magnetization. Remanent magnetization will be discussed later in more detail. 


\section{The Geomagnetic Field}

The Earth's main magnetic field, the geomagnetic field, is believed to be caused by a dynamo action produced by the circulation of charged particles in coupled convection cells located within the outer part of the Earth's core. Since these circulation patterns within the outer core change slowly with time, there is a slow, progressive, temporal change in the geomagnetic field called secular variation. This change is evident in the observed gradual rotation of the north magnetic pole around the geographic pole. Also, according to paleomagnetic studies, the polarity of the geomagnetic field occasionally reverses itself, at periods on the order of a few-hundred thousand years. This has been attributed to the change in dominance among the coupled convection cells.

In addition to the secular variations of the geomagnetic field, there are magnetic effects of external origin that change the field much more rapidly. These changes are due to magnetic fields induced by electrical currents in the ionized layer of the upper atmosphere. There are diurnal variations in the geomagnetic field that range in amplitude from about 20-80 nanoTesla (nT are the SI units for weak magnetic fields), with maximum variation at the polar regions. These variations are thought be controlled by the action of the solar winds and perhaps the moon on ionospheric currents. There are also far less regular and much stronger short-term variations in the geomagnetic field with amplitudes of up to $1000 \mathrm{nT}$. These disturbances are referred to as magnetic storms and are caused by intense solar activity.

At any point on the Earth's surface, the geomagnetic field can be characterized by its direction and intensity. Often, the parameters that are measured in the field include 
the magnetic declination $D$, the horizontal intensity $H$ and the vertical intensity $Z$. From these elements, all other parameters of the magnetic field can be calculated. These components are generally reported in SI units of nanoTesla. The Earth's magnetic field intensity ranges approximately $25,000 \mathrm{nT}$ at the magnetic equator to $70,000 \mathrm{nT}$ at the magnetic poles.

The International Geomagnetic Reference Field (IGRF) defines the theoretical undisturbed geomagnetic field at any point along the Earth's surface. Nearly $90 \%$ of the undisturbed field can be represented by a magnetic dipole at the center of the Earth inclined at about 11.5 degrees to the axis of rotation. The magnetic moment of this fictitious geocentric dipole can be calculated from the observed field. If this dipole is subtracted from the observed field, the residual field can be approximated by the effects of a second, smaller dipole. This process can be continued by fitting dipoles of ever decreasing moment until the observed geomagnetic field is simulated to any required degree of accuracy. This fitting process is carried out using spherical harmonic analysis and was used to compute the formula for the IGRF. Variations in the geomagnetic field can be determined by subtracting the IGRF from observed magnetic-field data. These variations are referred to as magnetic anomalies. 


\section{Rock Magnetism}

Most common rock-forming minerals have very small magnetic susceptibilities. Anomalies in the geomagnetic field are mainly caused by variations in the presence of a small proportion of magnetic minerals found in the underlying rocks. There are two geochemical groups that contain these minerals, the iron-titanium-oxygen group and the iron-sulfide group. The first group contains the mineral magnetite $\left(\mathrm{Fe}_{3} \mathrm{O}_{4}\right)$ and a solid solution between ilmenite $\left(\mathrm{FeTiO}_{3}\right)$ and hematite $\left(\mathrm{Fe}_{2} \mathrm{O}_{3}\right)$ (Blatt and Tracy, 1995). Magnetite and ilmenite are ferrimagnetic minerals and have a relatively high magnetic susceptibility. Hematite, on the other hand, is anti-ferromagnetic and does not usually give rise to any anomalies. The second group provides the mineral pyrrhotite $\left(\mathrm{FeS}_{1+\mathrm{x}}\right.$, $0<x<0.15$ ). The magnetic susceptibility of pyrrhotite is dependant upon the actual composition of the mineral and is also a ferrimagnetic mineral. Magnetite is by far the most common magnetic mineral, and the magnetic susceptibility of most rocks is proportional to the magnetite content. To a lesser extent, however, minerals such as ilmenite or pyrrhotite also give rise to a significant magnetic susceptibility.

Magnetite content in rocks can vary dramatically, which makes direct correlation between lithology and susceptibility very difficult. However, it is reasonable to classify the magnetic behavior of rocks according to their overall magnetite content. Basic igneous rocks have a relatively high magnetite content, which causes them to be highly magnetic. The proportion of magnetite in igneous rock tends to decrease with increasing silica content, making acid igneous rocks generally less magnetic than basic igneous rocks. Metamorphic rocks also vary greatly in their magnetite content. The abundance 
of iron and the partial pressure of oxygen, along with the degree of metamorphism, control the amount of magnetite and subsequently the degree of magnetic susceptibility that is formed in the rock. Sedimentary rocks are rarely magnetic, and most anomalies observed over sediment-covered areas are caused by underlying igneous or metamorphic basement, or by intrusions into the sediments. Other causes may include buried volcanic flows and man-made ferrous materials.

Any rock containing magnetic minerals may posses both an induced magnetization vector $J_{i}$ and a remanent magnetization vector $J_{r}$. Induced magnetization is a direct result of the present-day geomagnetic field and would be lost if the geomagnetic field could be removed. Natural remanent magnetization (NRM) is a permanent magnetization of a rock and is dependant upon the magnetic history of the rock. These two magnetizations may differ substantially in both magnitude and direction. The total magnetization of a rock $J$ is the vector sum of the induced magnetization and the remanent magnetization:

$$
\vec{J}=\vec{J}_{i}+\vec{J}_{r} .
$$

There are several mechanisms by which natural remanent magnetization forms in rocks. Primary remanent magnetization may be acquired as an igneous or volcanic rock solidifies and cools through the Curie temperature of its magnetic minerals in the presence of the contemporaneous geomagnetic field (thermoremanent magnetization, TRM). If the field is strong enough, the dipoles will permanently orient themselves across imperfections within the grains of the mineral, setting up a permanent magnetization. Another form of primary remanent magnetization occurs as magnetic 
particles of sediment align within the Earth's field during sedimentation (detrital remanent magnetization, DRM). Secondary remanent magnetization occurs later in the rock's history. Magnetic minerals may recrystallize or grow during diagenesis or metamorphism in a particular magnetic orientation (chemical remanent magnetization, CRM). Secondary remanent magnetization can also develop slowly in a rock standing in an ambient magnetic field as the domain magnetizations relax into the direction of the field (viscous remanent magnetization, VRM). Also, the removal of a strong external field, such as what occurs when lightning strikes, produces remanent magnetization in small areas (isothermal remanent magnetization, IRM). 


\section{Magnetic Anomalies}

All magnetic anomalies are superimposed on the geomagnetic field and are the result of variations in the presence of magnetic minerals in the near-surface crust. Common causes of magnetic anomalies include dikes, faulted, folded or truncated sills and lava flows, massive basic intrusions, metamorphic basement rocks and magnetite ore bodies. Magnetic anomalies may range in amplitude from a few tens of nanoTesla over deep metamorphic basement to several hundred nT over basic intrusions and may reach an amplitude of several thousand nT over magnetite ores.

The normal elements of the geomagnetic field at any one point are related by

$$
B^{2}=H^{2}+Z^{2}
$$

where $B$ is the total geomagnetic-field intensity and $H$ and $Z$ are the horizontal and vertical components of $B$, respectively (Figure 3a). If a magnetic anomaly is superimposed on the geomagnetic field, there will be a change $\Delta B$ in the strength of the total-field vector $B$. At any one point, the anomaly produces a vertical component $\Delta Z$ and a horizontal component $\Delta H$ at an angle $\alpha$ to $H$. Only the part of $\Delta H$ in the direction of $H, \Delta H^{\prime}$, will contribute to the change in $B$ (Figure $3 b$ )

$$
\Delta H^{\prime}=\Delta H \cos \alpha .
$$

A vector sum of the magnetic anomaly and the geomagnetic field at any one point (Figure 3c) is given by

$$
(B+\Delta B)^{2}=\left(H+\Delta H^{\prime}\right)^{2}+(Z+\Delta Z)^{2} .
$$

This equation simplifies to give

$$
\Delta B=\Delta Z(Z / B)+\Delta H^{\prime}(H / B)
$$


in geomagnetic element ratios, or with angular descriptions

$$
\Delta B=\Delta Z \sin I+\Delta H \cos I \cos \alpha
$$

where $I$ is the inclination of the geomagnetic field. Any volume of magnetic material in the Earth's crust can be considered as a continuous distribution of magnetic dipoles. At any point on the Earth's surface, the effects of all magnetic dipoles in the material can be summed to give a net change $\Delta B$ in the total geomagnetic field $B$.

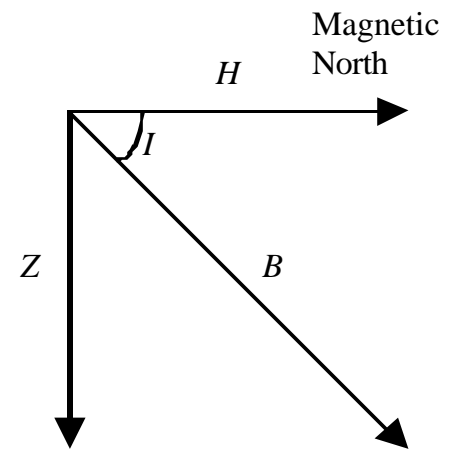

(a) section

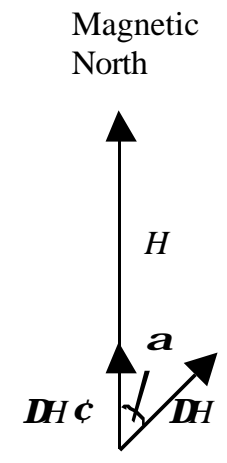

(b) plan

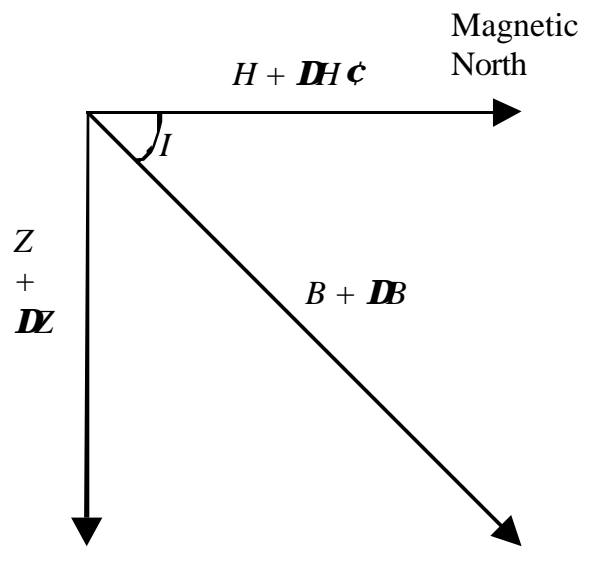

(c) section

Figure 3. Vector representation of the geomagnetic field with and without a superimposed magnetic anomaly. (adapted from Kearey and Brooks, 1991).

The magnetic dipoles in the material, excluding those in magnetic domains that exhibit remanent magnetization, will align with the present-day geomagnetic field by induction. The resulting magnetic anomaly produced by the magnetic material is defined by the total magnetization vector of the material, $J$. The amplitude of a magnetic anomaly is based on the magnitude of $J$, and the shape of the anomaly is affected by the direction of the $J$. 


\section{3-D DEPTH-TO-SOURCE METHODS}

Automated depth-to-source interpretation techniques available today rely on the fact that magnetic fields (and gravitational fields) are three-dimensional potential fields. Magnetic fields are conservative, meaning the work done in moving a unit positive pole is independent of the path traversed and depends only upon the end points. If the magnetic pole is eventually returned to its original position, the net energy expenditure is zero, regardless of the path followed. The work done on a unit-positive pole in bringing it from infinity by any path against a magnetic field is the magnetic scalar potential (Telford et al., 1990).

In previous discussion, the magnetic field strength, $H$, was defined as the force exerted on a unit positive pole due to a pole of strength $m$ at a distance $r$ from the pole (Equation 2). Accordingly, the magnetic potential $V$ of a unit positive pole due to a pole of strength $m$ at a distance $r$ from the pole is given by

$$
V=\frac{\grave{\iota}_{o} m}{4 \partial \grave{\imath}_{R} r}
$$

(Kearey and Brooks, 1991). Whereas the magnetic field strength is a vector quantity, having both magnitude and direction, a magnetic potential is a scalar, having magnitude only.

Removing the direction by representing the field as a scalar provides computational flexibility (Kearey and Brooks, 1991). The partial derivative of the 
magnetic potential in any direction is simply the magnetic field component in that direction

$$
H=-\nabla V
$$

(Telford et al., 1990). In addition to providing the magnetic field component in any direction, the magnetic potential satisfies Laplace's equation:

$$
\nabla^{2} V=0
$$

(Telford et al., 1990). This property of potential fields allows a magnetic field to be determined over any arbitrary surface if the field is known completely over another surface, assuming that the observed surface is outside of the sources. This is called continuation, and can be used to determine the field at a plane higher or lower than the plane of observation. This idea is the origin of all automated depth estimation techniques.

Many of the automated techniques available today are capable of rapidly calculating depth estimates from large grids of data. In most modern magnetic surveys, such as the one conducted for this study, magnetic field data are collected as a series of point values down a number of parallel lines over the survey area. The spacing between these point values, however, is not constant and there are usually large gaps between adjacent lines of data. A grid is an ordered array of magnetic field values at regularly spaced intervals. When a grid is created, the magnetic field values are interpolated between gaps in a series of survey points. A grid can be used to create visual representations of the magnetic field data such as contour maps, color images and shaded relief images. 
The three-dimensional automated depth-to-source interpretation techniques used in this study include Euler deconvolution (Thompson, 1982; Reid et al., 1990), the simple analytic signal (Nabighian, 1972, 1984; Roest et al., 1992), the horizontal gradient (Roest and Pilkington, 1993; Phillips, 2001), the enhanced analytic signal (Hsu et al., 1996; Hsu et al., 1998) and the local wavenumber (Thurston and Smith, 1997; Smith et al., 1998). These techniques were applied to gridded magnetic anomaly data over specific anomalies in the Tucson basin, and both depth and horizontal location of the magnetic contacts were determined. 


\section{Euler Deconvolution}

The Euler deconvolution method relies on Euler's homogeneity equation:

$$
\left(x-x_{0}\right) \frac{\partial T}{\partial x}+\left(y-y_{0}\right) \frac{\partial T}{\partial y}+\left(z-z_{0}\right) \frac{\partial T}{\partial z}=N(B-T)
$$

where $\left(x_{0}, y_{0}, z_{0}\right)$ is the position of a magnetic source whose total field $T$ is detected at $(x, y, z)$. The total field has a regional value of $B$. The degree of homogeneity $N$ is interpreted as a structural index (SI) (Thompson, 1982; Reid et al., 1990).

Unlike many other computer-assisted methods before it, Euler deconvolution does not assume any particular geologic model. The method can be applied and interpreted even when the geology cannot be properly represented by prisms or dikes. Also, unlike previous methods, this method can be applied directly to gridded data (Thompson, 1982).

The structural index in Euler's equation is a measure of the rate of change with distance of a field. For a point dipole, the magnetic field strength decreases as the inverse cube of the distance, giving an index of three. An effective vertical lne source, such as a narrow vertical pipe, gives rise to an inverse square field falloff and an index of two. Extended bodies have indices ranging from zero, like that of an infinite sheet, to three (Reid et al., 1990). Since Euler deconvolution does not make any assumptions about the geologic model, the user must choose the structural index that best fits the data.

For magnetic contacts, Thompson (1982) suggested that the index be less than 0.5. Reid et al. (1990) showed that this choice of an index for magnetic contacts leads to underestimates of depth. They showed that the value for a sloping contact is zero, 
provided that an offset $A$ is introduced, and introduced a revised form of Euler's equation:

$$
\left(x-x_{0}\right) \frac{\partial T}{\partial x}+\left(y-y_{0}\right) \frac{\partial T}{\partial y}+\left(z-z_{0}\right) \frac{\partial T}{\partial z}=A
$$

where $A$ incorporates amplitude, strike and dip factors which cannot be separated easily (Reid et al., 1990).

The three-dimensional forms of Euler's equation (Equations 18 or 19) are easily applied to gridded data. The first step is to calculate the total-field gradient in three orthogonal directions, äx, äy and äz (if these quantities are not already measured in the field) (Reid et al, 1990). In most cases, the total magnetic field $T$ is measured and plotted as a two-dimensional grid. The horizontal gradients are found by using a finitedifference approach. A square $3 \times 3$ data window is passed over each grid point and the average differences between corresponding grid points in the $x$ and $y$ directions are determined (Cordell and Grauch, 1985). The vertical derivative is calculated in the frequency domain using a Fourier transformation. In the frequency domain, the Hilbert transform relationship allows the vertical derivative to be calculated when horizontal derivatives in two perpendicular directions are available (Nabighian, 1984; Roest et al., 1992).

The second step is to place a moving square data window of size $3 \times 3$ or greater within the grids of the gradient values and of the field values. For a non-zero structural index, all points in the data window are used to solve Euler's equation (Equation 18) for the source position $\left(x_{0}, y_{0}, z_{0}\right)$ and a background value $B$ (Reid et al., 1990). Euler's 
equation is solved by creating an over-determined set of linear equations. There are four unknowns in the equation $\left(x_{0}, y_{0}, z_{0}\right.$ and $\left.B\right)$ and a minimum of nine linear equations (resulting from the $3 \times 3$ data window). The nine equations and the four unknowns are solved using a least-squares procedure. The least-squares solution of the over-determined set of equations also yields estimates of the standard deviation of the parameter $z_{0}$. This quantity is treated as an "error bar" on the depth estimates (Thompson, 1982). For a structural index of zero, the modified Euler's equation (Equation 19) is used and the source position and arbitrary offset value $A$ are solved (Reid et al., 1990).

All possible data window positions, including overlaps, are used to solve Euler's equation. Only those solutions that fall below a chosen threshold of the standard deviation of $z_{0}$, usually $15 \%$ or less, are preserved. All preserved solutions are then plotted in plan view, using symbols with sizes proportional to depth $z$ (Reid et al., 1990).

The choice of a proper structural index is crucial in order to attain correct depths and converging solutions over magnetic contacts. Reid et al. (1990) showed that an index that is too low gives depths that are too shallow, and an index that is too high gives estimates that are too deep. They also found that even if the index is correct, depth estimates for high-index sources are more precise that those for low-index sources. Gross structural trends, however, can still be outlined despite a poor choice of the index.

Real data sets are likely to contain anomalies from sources with various structural indices. Therefore, it is necessary to solve for a range of indices and to plot the results for each index. The correct index for a particular feature gives the best solution clustering and consequently the best depth estimates (Reid et al., 1990). 
The choice of the data-window size also greatly affects solutions to Euler's equation. If anomalies arising from more than one source occupy any given data window, poor fit statistics cause the solution to be rejected. It is then reasonable to keep the search window as small as possible. Conversely, broad anomalies arising from deep sources are poorly represented in a small data window, and unreliable estimates of depth and position of source are likely (Reid et al., 1990). 


\section{Simple Analytic Signal}

The simple analytic signal $A$, or "energy envelope" of a three-dimensional magnetic anomaly is defined as

$$
A(x, y)=\left(\frac{\partial T}{\partial x} \hat{x}+\frac{\partial T}{\partial y} \hat{y}+i \frac{\partial T}{\partial z} \hat{z}\right)
$$

where $T$ is the total magnetic field, $i$ is the imaginary number and $\hat{x}, \hat{y}, \hat{z}$ are unit vectors in the principle Cartesian coordinates. The real and imaginary parts of Equation 20 form a Hilbert transform pair, which allows the vertical derivative to be easily calculated in the frequency domain when horizontal derivatives in two perpendicular directions are available (Nabighian, 1984; Roest et al., 1992). The absolute value, or amplitude, of the simple analytic signal is given by

$$
|A(x, y)|=\sqrt{\left(\frac{\partial T}{\partial x}\right)^{2}+\left(\frac{\partial T}{\partial y}\right)^{2}+\left(\frac{\partial T}{\partial z}\right)^{2}}
$$

(Roest et al., 1992). This function acts as the "envelope" of all possible phase shifts of an observed anomaly and peaks over magnetic contacts, regardless of the regional magnetic field direction, the source magnetization and the dip of the contact (Roest et al., 1992) (Figure 4). The contacts, however, must be isolated and effectively two-dimensional. That is to say they have an effective infinite strike length (Thurston and Smith, 1997). The maximum amplitude of the analytic signal is dependant upon the intensity of magnetization, the inclination of the Earth's field and the strike of the geologic boundary. This can make interpretation difficult if the geologic boundary is close to the magnetic equator or when its strike is near parallel to the magnetic north (Roest et al., 1992). 

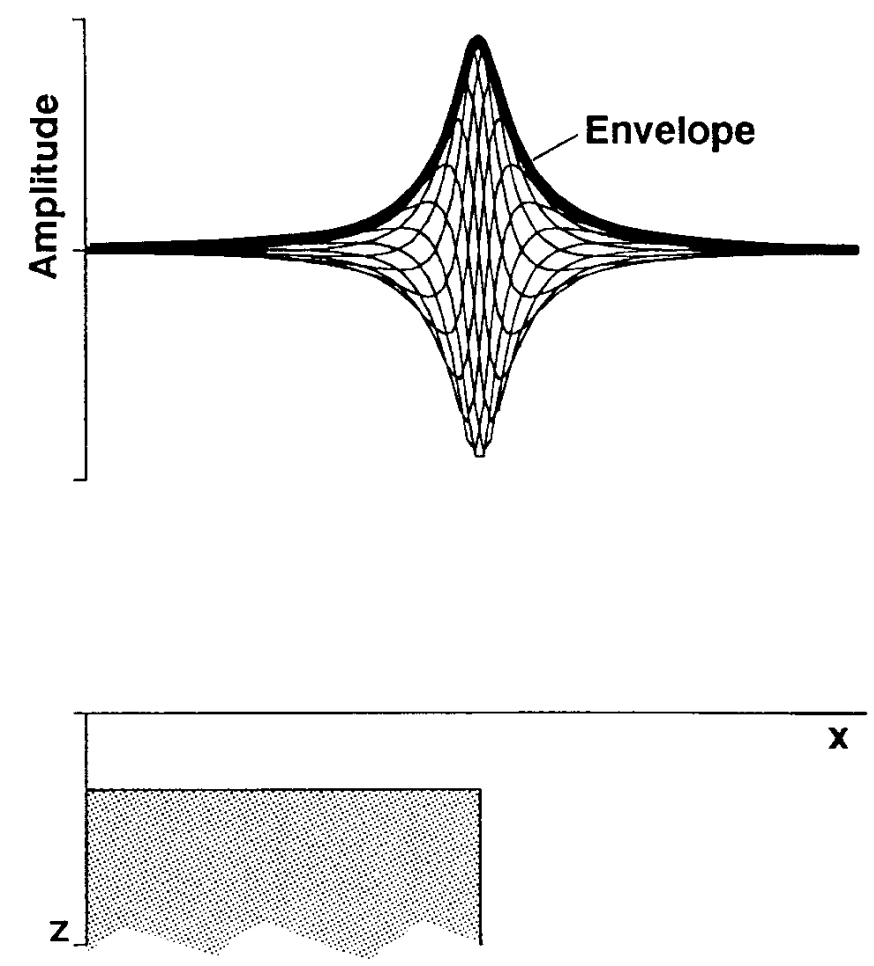

Figure 4. The two-dimensional simple analytic signal amplitude over a magnetic contact. This function acts as the envelope of all possible phase shifts (i.e. magnetization directions) of an anomaly. The individual curves inside the envelope represent different phase angles shifted in steps of 30 degrees (from Roest, et al., 1992).

Since the amplitude of the simple analytic signal peaks over magnetic contacts, it can be used to find horizontal locations and depths of magnetic contacts. This method of contact location is easily applied to gridded data. The first step is to calculate a grid of the analytic signal amplitude from a magnetic anomaly grid. The field gradients of the magnetic anomaly grid in two orthogonal horizontal directions, äx and äy, are calculated using a finite-difference approach. A square $3 \times 3$ data window is passed over each grid point, and the average differences between corresponding grid points in the $x$ and $y$ directions are determined (Corde 
calculated in the frequency domain, making use of the Hilbert transform relationship. The amplitude of the analytic signal is calculated at each grid point using Equation 21, and a new grid is created.

The second step is to find crests in the analytic signal amplitude. The peak values are located by placing a square data window of size $3 \times 3$ within the newly created grid and searching for local maxima. Each grid intersection, except for marginal rows and columns, is compared with its eight nearest neighbors in four directions (along the row, column and both diagonals) to find any local maxima that are present. If a local maximum is found, a more precise horizontal location and the magnitude of the maximum are found by interpolating a second-order polynomial through the trio of points. The horizontal location $x_{\max }$ of the maximum relative to the position of the grid intersection point, $g_{\mathrm{i}, \mathrm{j}}$ is given by

$$
x_{\text {max }}=-\frac{b d}{2 a}
$$

where

$$
\begin{aligned}
& a=\frac{1}{2}\left(g_{i-1, j}-2 g_{i, j}+g_{i+1, j}\right), \\
& b=\frac{1}{2}\left(g_{i+1, j}-g_{i-1, j}\right)
\end{aligned}
$$

and $d$ is the distance between grid intersections. The magnitude $g_{\max }$ of the analytic signal amplitude at $x_{\max }$ is given by

$$
g_{\text {max }}=a x_{\text {max }}^{2}+b x_{\text {max }}+g_{i, j} .
$$


The largest $g_{\max }$ and its corresponding $x_{\max }$ are chosen for grid intersections with more than one local maximum (Blakely and Simpson, 1986). Another criteria for selecting the maxima is to use an index, $N$, which is defined as the number of valid maxima found in the $3 \times 3$ data window. The $N$ index gives an indication of the linearity of a maximum. If $\mathrm{N}$ is 1 , the anomaly is linear, but tilted; if $\mathrm{N}$ is 4 , the maximum is a local peak. Blakely and Simpson (1986) found that, in general, indices 2 and 3 were most useful.

The third step is to calculate the depths to the contacts once the locations of the crests in the analytic signal amplitude have been determined. Phillips (1997) uses a similar approach to find maxima, but uses a $5 \times 5$ data window to locate both crests in the analytic signal amplitude and their strike directions. An algorithm that finds the azimuth with the narrowest cross-section through the maximum is assumed to be perpendicular to the strike of a linear anomaly (Roest et al, 1992). Once the crests and their associated strike directions are known, data within the window and within a belt perpendicular to the strike can be used to determine the depth of the contact by performing a least-squares fit to the theoretical shape of the amplitude of the two-dimensional simple analytic signal over a contact (Phillips, 2001). This curve is given by

$$
|A(h)|=\frac{\alpha}{\sqrt{h^{2}+d^{2}}}
$$

with $h$ being the horizontal distance to the contact, $d$ the depth to the top of the contact and $\alpha$ a constant (Nabighian, 1972). Alpha is the product of the susceptibility contrast across the magnetic contact, the Earth's magnetic field strength and inclination, and the angle between magnetic north and the positive $x$-axis. The least-squares fit provides an 
estimate of both the depth and its standard error, which can be expressed as a percentage of the depth. A poor fit may be an indication of poor data quality or the interference of multiple anomalies (Roest et al., 1992). Phillips (1997) recommends retaining only those solutions with standard errors of $15 \%$ or better in the final interpretation.

An assumption of thick sources establishes that the depth estimates obtained using Equation 24 represent minimum depths (i.e. depths to the top of infinitely thick sources). However, an assumption of thick sources is not always valid. Due to the dipolar nature of magnetic fields, anomaly interference from the bottoms of magnetic contacts increases as source thickness decreases. Depth calculations that are made over sources that are relatively thin will result in incorrect depth estimates. It is possible to eliminate these interference effects by applying a pseudo-gravity transformation. The pseudo-gravity transformation relies on Poisson's relation, which shows that magnetic fields can be transformed into gravitational fields, and vice versa, for bodies in which the ratio of intensity of magnetization to density remains constant (refer to Baranov, 1957 for more on pseudo-gravity transforms). This transform is a linear filter applied in the frequency domain that transforms the magnetic anomaly observed over a magnetization distribution into the gravity anomaly that would be observed if there were a similar distribution of mass density (Blakely and Simpson, 1986). Since gravitational fields are not dipolar, gravity anomalies are due to the effects of he entire source body, not just its edges (i.e. top and bottom). If the analytic signal amplitude of the pseudo-gravity field is used to solve Equation 24, the depth estimates will represent maximum depths to the top of 
magnetic sources (Phillips, 1997). For an infinitely thin source, this would be the same as the depth to the top of the source body. 


\section{Horizontal Gradient}

The horizontal gradient method is the simplest approach to estimating magnetic contact locations and depths, because it does not require calculating vertical derivatives. Since it does not require the calculating vertical derivatives, it requires the greatest number of assumptions about the sources (Grauch and Cordell, 1987; Phillips, 2001). If $T$ is the total magnetic field and $x$ and $y$ represent two orthogonal horizontal directions, then the magnitude of the horizontal gradient $H G M$ is defined as

$$
H G M=\sqrt{\left(\frac{\partial T}{\partial x}\right)^{2}+\left(\frac{\partial T}{\partial y}\right)^{2}}
$$

(Cordell and Grauch, 1985). The horizontal gradient magnitude is used as an approximation of the analytic signal amplitude over magnetic contacts (Roest and Pilkington, 1993). This function peaks over magnetic contacts under the following assumptions: the regional magnetic field is vertical, the source magnetizations are vertical, the contacts are vertical, the contacts are isolated and the sources are thick. If any of the first four assumptions are violated, the peaks are shifted away from the magnetic contacts. If the last assumption, that of thick sources, is violated, secondary peaks parallel to the contacts can appear (Roest and Pilkington, 1993; Phillips, 2001).

In order to satisfy the first two assumptions, it is necessary to apply a standard phase shift to the data known as reduction to the pole. Reduction to the pole can be done with a Fourier transform and changes the phase of magnetic anomalies to represent anomalies arising from a vertical regional magnetic field (refer to Baranov, 1957 for 
more on reduction to the pole). Assuming the sources do not exhibit strong remanent magnetization, the source magnetizations will also become vertical (Phillips, 2001).

To apply this method to gridded data, the field gradients in two orthogonal directions, äx and äy, are calculated using a finite-difference approach. Equation 25 is then solved for the magnitude of the horizontal gradient at each grid point, and a new grid containing the magnitude of the horizontal gradient is created. Crests in the horizontal gradient magnitude are found by passing a $5 \times 5$ data window over the $H G M$ grid and searching for local maxima in the same manner as in the simple analytic signal method.

When a crest is found and its local strike direction is determined, the source depth and its standard error are estimated by a least-squares fit perpendicular to strike to the theoretical shape of the two-dimensional horizontal gradient magnitude over a contact. This curve is given by

$$
H G M=\frac{\alpha d}{h^{2}+d^{2}}
$$

with $h$ being the horizontal distance to the contact, $d$ the depth to the top of the contact and $\alpha$ a constant defined by the susceptibility contrast across the magnetic contact, the Earth's magnetic field strength and inclination, and the angle between magnetic north and the positive $x$-axis (Phillips, 2001). This function has a bell shape over contacts that is similar to that of the analytic signal (Roest and Pilkington, 1993) (Figure 5). 

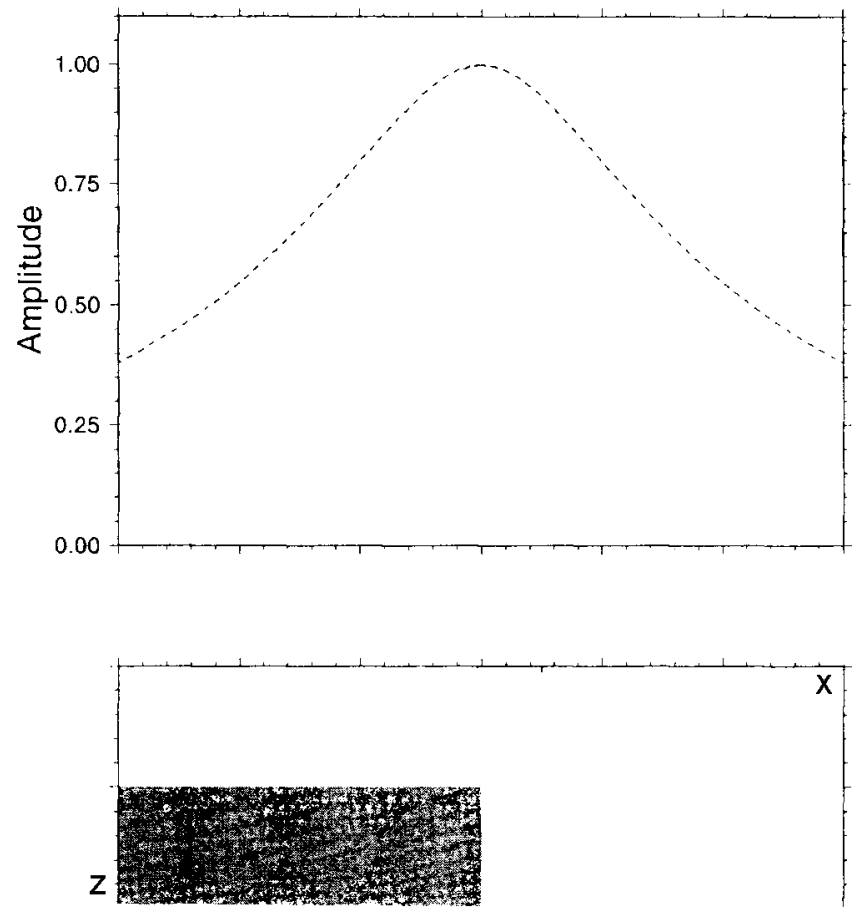

Figure 5. The two-dimensional horizontal gradient magnitude over a magnetic contact (modified from Roest and Pilkington, 1993).

This method also provides depth estimates representing minimum depths with the assumption of thick sources. As with the simple analytic signal method, it is possible to assume very thin sources by using a pseudo-gravity transformation in lieu of a reduction to the pole. If the analysis is done on the pseudo-gravity field, the depth estimates represent maximum depths (Phillips, 1997). 


\section{Enhanced Analytic Signal}

Since potential-field data correspond to the superposition of effects from all causative sources, the determination of geologic boundaries usually suffers from nearby source interference. This leads to offset magnetic contact locations and incorrect depth estimates (Hsu et al., 1996). To reduce interference effects, it is necessary to increase anomaly resolution. The simple analytic signal may not detect geologic boundaries and their proper depths sufficiently, because its bell-shaped function is broad over the boundaries. Therefore, it has been suggested that taking high-order derivatives of the analytic signal will increase resolution and decrease interference effects (Nabighian, 1972; Hsu et al., 1996).

The $n^{\text {th }}$-order enhanced analytic signal $A_{n}$ is defined as

$$
A_{n}(x, y)=\frac{\partial}{\partial x}\left(\frac{\partial^{n} T}{\partial z^{n}}\right) \hat{x}+\frac{\partial}{\partial y}\left(\frac{\partial^{n} T}{\partial z^{n}}\right) \hat{y}+i \frac{\partial}{\partial z}\left(\frac{\partial^{n} T}{\partial z^{n}}\right) \hat{z}
$$

where $T$ is the total magnetic field, $i$ is the imaginary number and $\hat{x}, \hat{y}, \hat{z}$ are unit vectors in the principle Cartesian coordinates. The amplitude of the $n^{\text {th }}$-order enhanced analytic signal is then given by

$$
\left|A_{n}(x, y)\right|=\sqrt{\left[\frac{\partial}{\partial x}\left(\frac{\partial^{n} T}{\partial z^{n}}\right)\right]^{2}+\left[\frac{\partial}{\partial y}\left(\frac{\partial^{n} T}{\partial z^{n}}\right)\right]^{2}+\left[\frac{\partial}{\partial z}\left(\frac{\partial^{n} T}{\partial z^{n}}\right)\right]^{2}}
$$

(Hsu et al., 1996). All orders of this function peak over magnetic contacts, regardless of the regional magnetic field direction, the source magnetization and the dip of the contact. 
The theoretical shape of the amplitude of the two-dimensional enhanced analytic signal over a contact is given by

$$
\left|A_{n}(h)\right|=\sqrt{\frac{n ! \alpha^{2}}{\left(h^{2}+d^{2}\right)^{n+1}}}
$$

with $h$ being the horizontal distance to the contact, $d$ the depth to the top of the contact and $\alpha$ a constant defined by the susceptibility contrast across the magnetic contact, the Earth's magnetic field strength and inclination, and the angle between magnetic north and the positive $x$-axis (Nabighian, 1972).

Hsu et al. (1998) suggested a series of equations to estimate the depths to magnetic contacts that are based on amplitude ratios of the peak values of different orders of the enhanced analytic signal. These equations differ from one another by using different initial models. Changing the initial model assumptions can improve the accuracy of depth estimation (Hsu et al., 1998). Three initial models and their corresponding equations proposed by Hsu et al. (1998) include a step model with infinite thickness, a vertical-step model with finite thickness and a dike model. The complete derivations of the following equations can be seen in Hsu et al. (1998).

For a two-dimensional step-like structure, the depth $d$ can be estimated from the peak of $n^{\text {th }}$-order enhanced analytic signal $A_{n}$ in three ways:

$$
\begin{aligned}
& d=\frac{1}{c_{1}}, \\
& d=\sqrt{\frac{2}{c_{2}}}
\end{aligned}
$$




$$
d=\frac{2 c_{1}}{c_{2}}
$$

where

$$
c_{1}=\left|\frac{A_{1}}{A_{0}}\right| \quad \text { and } \quad c_{2}=\left|\frac{A_{2}}{A_{0}}\right|
$$

(Hsu et al., 1998). The depth estimates from the above equations are only valid when the bottom surface of the step model is at infinity. Since this assumption is not realistic, some error will result. However, the error generally decreases as the thickness of the step model increases (Hsu et al., 1998).

For the second model, a two-dimensional step-like structure with finite thickness $t$ and a vertical-dipping face, the maximum amplitude of the analytic signal is given by the difference between a shallow, infinite step with depth $d$ and a deep, infinite step with depth $l$. These unknown parameters can be solved for with the following equations:

$$
d=\frac{c_{1}-\sqrt{2 c_{2}-3 c_{1}^{2}}}{2 c_{1}^{2}-c_{2}}
$$

and

$$
l=\frac{d}{c_{1} d-1}
$$

where

$$
c_{1}=\left|\frac{A_{1}}{A_{0}}\right| \quad \text { and } \quad c_{2}=\left|\frac{A_{2}}{A_{0}}\right|
$$


(Hsu et al., 1998). In order for the depth $d$ to be a meaningful solution, the maximum of the analytic signal must be due to a near-vertical step-like structure. The following inequality must be true to satisfy the assumption:

$$
2 c_{2}-3 c_{1}^{2}>0
$$

(Hsu et al., 1998). The estimation of $l$ is not very stable, however, due to the problem of a poor signal-to-noise ratio (Hsu et al., 1998).

The third model is that of a dike. It was discovered that if the half-width between two edges is less than their depth, two maxima of the simple analytic signal amplitude from the two nearby parallel edges will merge to one (Atchuta Rao et al., 1981). Since potential-field data represent the superposition of effects from multiple causative sources, a dike model can be egarded as the difference between two parallel step models with a fixed horizontal offset (Hsu et al., 1998). Hsu et al. (1998) showed that the analytic signal amplitudes are symmetric about the center of a dike and are independent of the dipping angle. The depth $d$ and the half-width $w$ of a dike model can be estimated from

$$
d=\frac{c_{1}}{2 c_{1}^{2}-c_{2}}
$$

and

$$
w=\sqrt{\frac{2 d}{c_{1}}-d^{2}}
$$

respectively, where

$$
c_{1}=\left|\frac{A_{1}}{A_{0}}\right| \quad \text { and } \quad c_{2}=\left|\frac{A_{2}}{A_{0}}\right|
$$


(Hsu et al., 1998). If the half-width is determined to be less than zero, then the peak of the analytic signal amplitude is not centered over a dike-like structure. In this case, a step model should be used for estimating the depth (Hsu et al., 1998).

Hsu et al. (1998) found that higher order analytic signal ratios are generally less sensitive to finite thickness (Figure 6). They also found that it was best to use amplitude values of the lower-order analytic signals at the locations defined by the maximum amplitudes of second-order enhanced analytic signal, since higher-order analytic signals suffer the least from interference effects (Hsu et al., 1996).

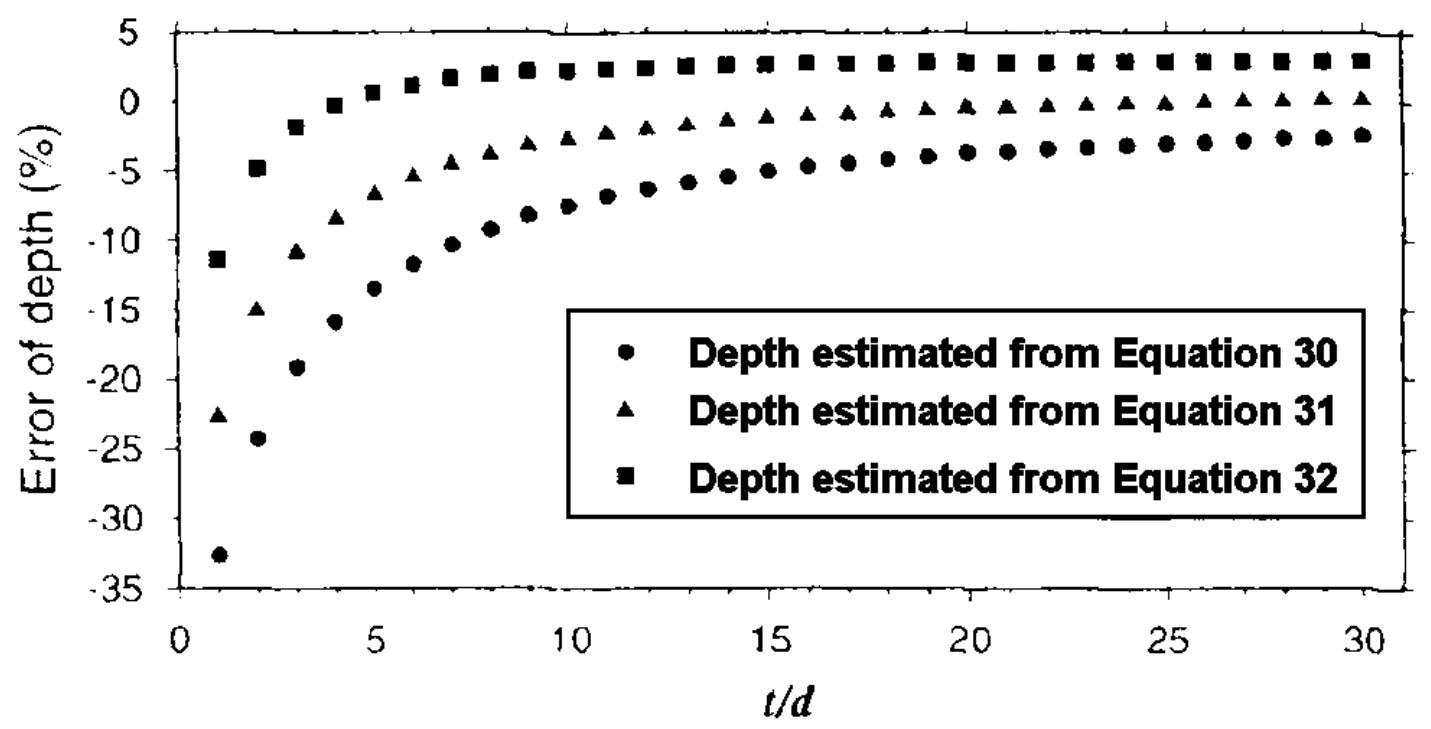

Figure 6. Plot of the ratio of thickness $t$ to depth $d$ below the observation level versus estimation error of depth $\boldsymbol{d}$ due to a vertical step model enhanced analytic signal approach (adapted from Hsu et al., 1998). 


\section{Local Wavenumber}

The local wavenumber comes from The Source Parameter Imaging (SPI) method (Thurston and Smith, 1997), which extends the theory of the analytic signal by computing three complex attributes from which source parameters can be calculated. In the case of spatial magnetic data, these attributes include the local amplitude (analogous to the analytic signal amplitude), local phase and local wavenumber. The local wavenumber $k$ is defined as the rate of change of the local phase $\theta$ of the total field $T$ with respect to the horizontal $(x$ and $y$ ) directions and can be expressed as

$$
k=\sqrt{\left(\frac{\partial \theta}{\partial x}\right)^{2}+\left(\frac{\partial \theta}{d y}\right)^{2}}
$$

where

$$
\theta=\tan ^{-1}\left(\frac{\frac{\partial T}{\partial z}}{\sqrt{\left(\frac{\partial T}{\partial x}\right)^{2}+\left(\frac{\partial T}{\partial y}\right)^{2}}}\right)
$$

(Thurston and Smith, 1997).

The local wavenumber, like the analytic signal, peaks over magnetic contacts and is independent of the regional magnetic field direction, the source magnetization and the dip of the contact (Thurston and Smith, 1997). The theoretical shape of the twodimensional local wavenumber over a contact is given by

$$
k=\frac{d}{h^{2}+d^{2}}
$$


with $h$ being the horizontal distance to the contact and $d$ the depth to the top of the contact (Figure 7). From this equation it, it is easily seen that directly over a contact the depth $d$ to the top of the contact with infinite depth extent is the inverse of the peak value of the local wavenumber (Thurston and Smith, 1997).
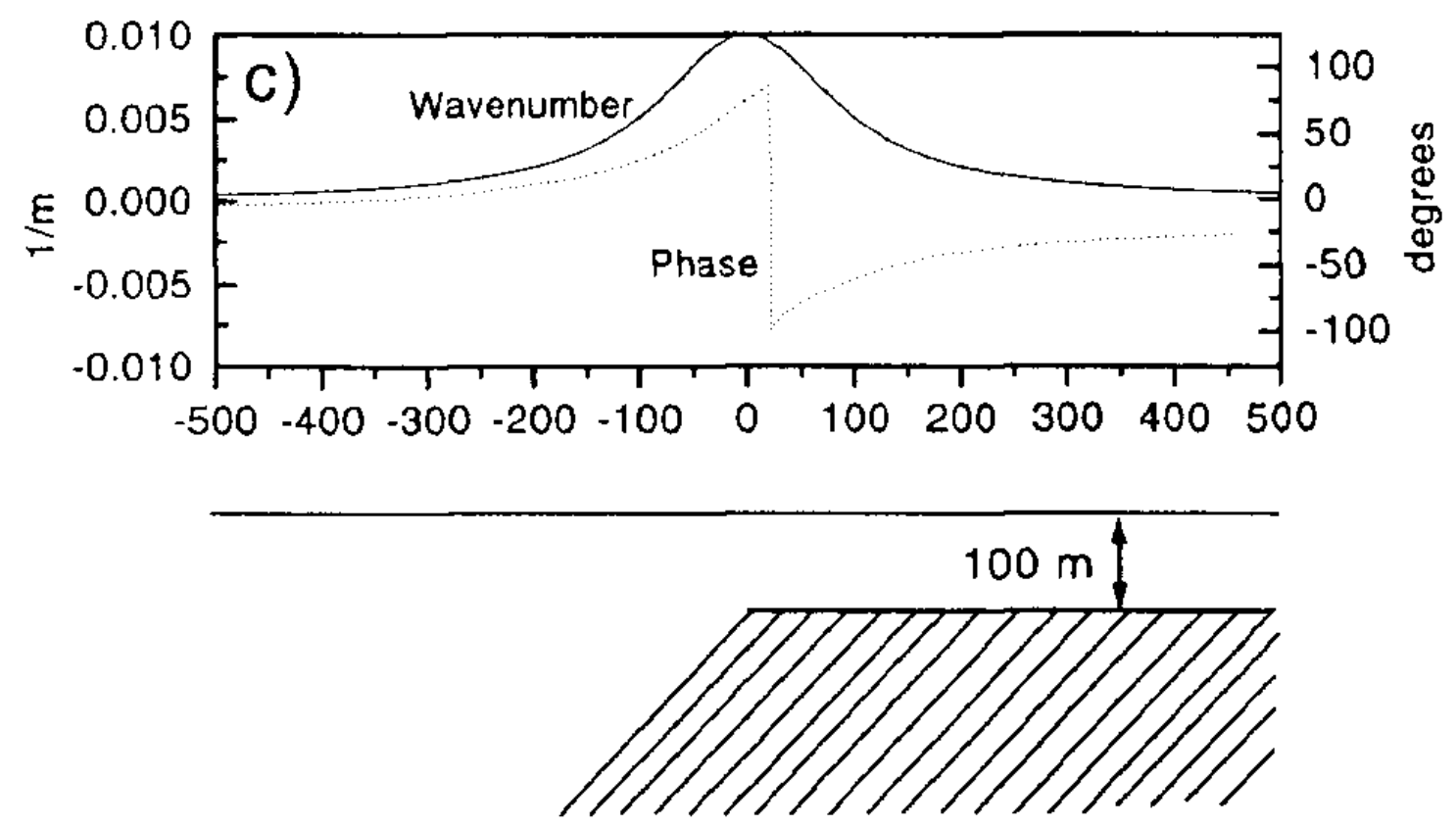

Figure 7. The two-dimensional local wavenumber over a magnetic contact (adapted from Thurston and Smith, 1997).

The local wavenumber method, like the previous methods, assumes the initial model of a dipping contact with infinite thickness. Phillips (1997) suggested that taking the vertical integral of the total magnetic field before calculating the local wavenumber would improve depth results for contacts with a finite thickness. Smith et al. (1998) discovered, however, that taking second order derivatives of the magnetic field could be useful. They introduced a higher order, or enhanced local wavenumber $k^{\prime}$ that is defined as 


$$
k^{\prime}=\sqrt{\left(\frac{\partial \boldsymbol{\theta}_{2}}{\partial x}\right)^{2}+\left(\frac{\partial \boldsymbol{\theta}_{2}}{d y}\right)^{2}}
$$

where

$$
\theta_{2}=\tan ^{-1}\left(\frac{\frac{\partial^{2} T}{\partial z^{2}}}{\sqrt{\left(\frac{\partial^{2} T}{\partial z \partial x}\right)^{2}+\left(\frac{\partial^{2} T}{\partial z \partial y}\right)^{2}}}\right)
$$

This function also peaks over the magnetic contacts. The only difference between $k$ and $k^{\prime}$ is a change in the amplitude factor, which is related to the structural index of the source body. A model independent local wavenumber can be calculated by subtracting $k$ from $k^{\prime}$. The depth $d$ to the magnetic contact can then be calculated by taking the inverse of this value:

$$
d=\frac{1}{k^{\prime}-k}
$$

(Smith et al., 1998). These depths are completely independent of any initial models, assuming the sources are effectively two-dimensional (Smith et al., 1998).

This method can be applied to gridded data in the same fashion as the other methods. Higher order derivatives are calculated in the frequency domain. The appropriate equation is solved for the local wavenumber at each grid point, and a new grid containing the local wavenumber is created. Crests in the local wavenumber are then found by passing a $5 \times 5$ data window over the newly created grid and searching for local maxima. When a crest is found and its local strike direction is determined, the 
source depth and its standard error are estimated by a least-squares fit perpendicular to strike to the theoretical shape of the two-dimensional local wavenumber over a contact. 


\section{DATA ACQUISITION AND PROCESSING}

\section{Instrumentation \& Procedure}

The data used in this project comes from Sander Geophysics Limited (SGL) (Desmons, 1998). SGL conducted a high-resolution aeromagnetic survey over the Tucson basin in Pima County, Arizona for the United States Geological Survey (USGS). The survey was flown in the fall of 1997, with the first production flight taking place on November 26, 1997 and the last one on December 19, 1997. Thirty-four flights, totaling 20,819 line-kilometers (12,936 miles) were required to complete the survey.

The survey area extended from the city of Tucson in the north to the town of Green Valley in the south (Figure 8). The corner coordinates that define the boundaries of the survey block are as follows: latitude $31 \mathrm{~N}^{\prime} 5^{\prime} 00^{\prime \prime}$, longitude $-111 \mathrm{~W} 05^{\prime} 00^{\prime \prime}$; latitude 32N20'00", longitude -111W05'00"; latitude 32N20'00", longitude -110W35'00"; latitude 31N45'00", longitude -110W35'00". These coordinates are given in the North American Datum 1927 (NAD-27), which uses the Clarke spheroid of 1866 to represent the shape of the Earth. The origin of this datum is a point on the Earth referred to as Meades Ranch located in Kansas. The survey consisted of 433 traverse lines oriented east-west and 5 orthogonal control lines. The flight line spacing was 150 meters, with control lines having a spacing of 12 kilometers. The data were collected with a draped survey, with an optimum survey ground clearance of 152 meters. 


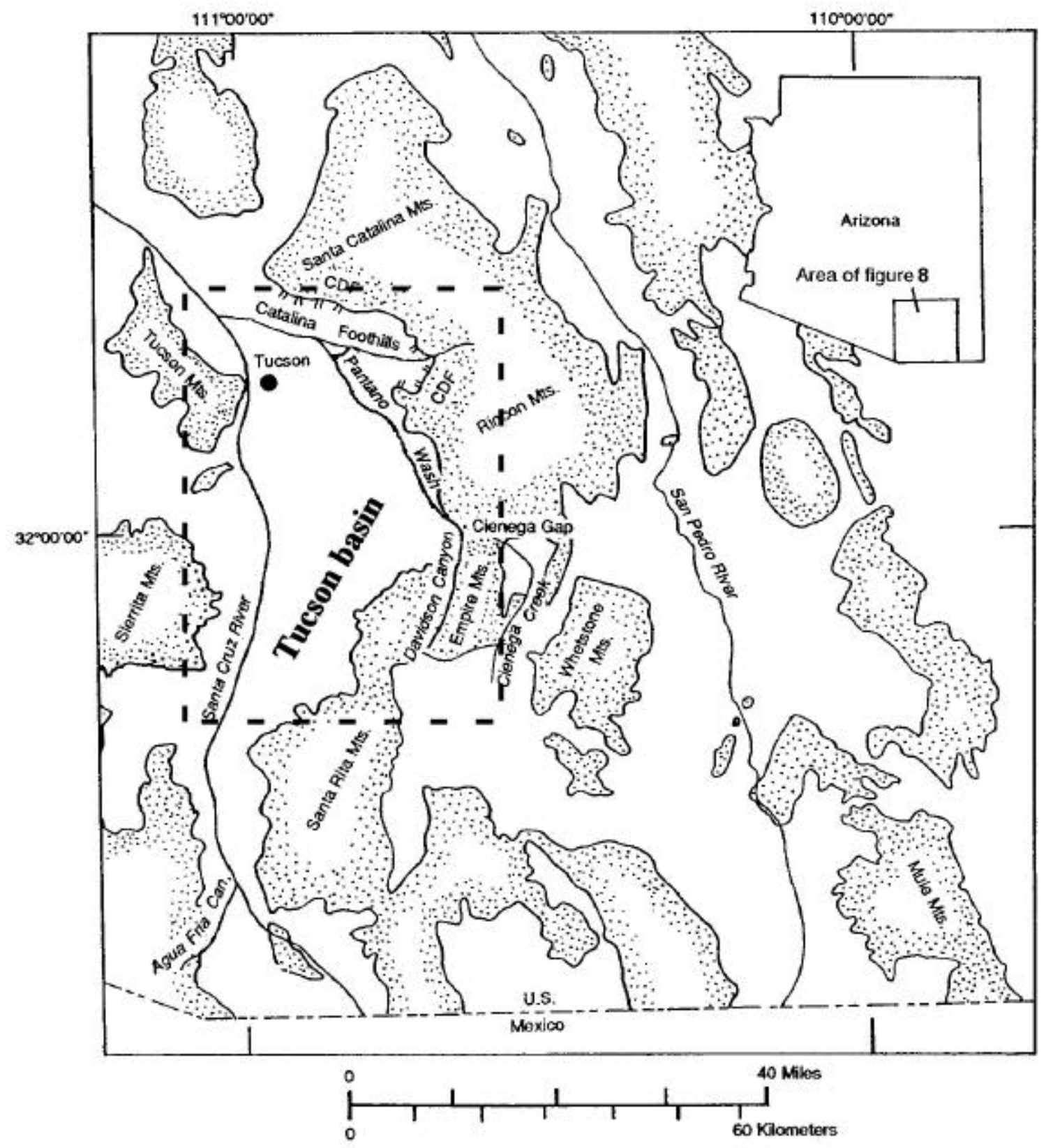

Figure 8. Aerial extent of aeromagnetic survey (within dashed lines) conducted over the Tucson basin by Sander Geophysics, Ltd (modified from Houser and Gettings, 2000). 
Both a ground and an airborne system, consisting of non-oriented optically pumped cesium split-beam sensors (refer to Telford et al., 1990 for more on optically pumped magnetometers) manufactured by Geometrics (Geometrics Model G-822A), were used during the survey. The magnetometers have a sensitivity of $0.005 \mathrm{nT}$ or better and an operating range of 15,000 to $100,000 \mathrm{nT}$. Sensor noise is less than $0.02 \mathrm{nT}$. The total-field magnetic measurements were digitally recorded at intervals of 0.1 second (10 $\mathrm{Hz})$ in the airborne system and 0.5 second $(2 \mathrm{~Hz})$ in the ground system.

To compensate for the magnetic field created by the aircraft, an RMS compensator (RMS Model AADC 4000 MkII) was used. The compensator is a fully automatic, 27-term compensator system utilizing a 3-axis fluxgate magnetometer for heading information. Magnetic information was output at a minimum of 0.1-second intervals $(10 \mathrm{~Hz})$, with a resolution of $0.001 \mathrm{nT}$. The system provided a complete realtime compensation of the aircraft maneuver noise.

Navigation and flight path recovery were provided by SGL's navigation system, GPSNav. The system utilized a NovAtel GPSCard 3951R 12-channel receiver mounted in a 486-based navigation computer with a sampling rate of 1.0 second $(1 \mathrm{~Hz})$. The navigation system provided essential positional data, for both the ground and airborne magnetometers.

Altimeter and ground clearance data were recorded using both an active radar system and a barometric pressure system. The TRT radar altimeter has a resolution of 1 meter, an accuracy of $2 \%$ and a range of 1 to 2,400 meters. The barometric pressure 
system has a resolution of 2 meters, an accuracy of $+/-4$ meters and a range of 1 to 9,100 meters.

The following parameters were recorded during the course of the survey: aircraft altitude as measured by the barometric altimeter at intervals of $0.25 \mathrm{~s}$; terrain clearance provided by the radar altimeter at intervals of $0.25 \mathrm{~s}$; a continuous video tape record of the terrain passing below the aircraft; airborne GPS positional data (latitude, longitude, height, time and raw range from each satellite being tracked), recorded at intervals of 1.0s; time markers synchronously impressed on the video and digital data; airborne total magnetic field recorded with a 0.1 s sampling rate; ground total magnetic field recorded with a $0.5 \mathrm{~s}$ sampling rate; ground based GPS positional data, recorded at intervals of $1.0 \mathrm{~s}$. The combined magnetic/GPS ground station was installed at the airport near the hangar. The NAD-27 coordinates of the ground station were latitude 32N24'22.34", longitude 111W12'57.13". 


\section{Data Reduction}

All data reduction was performed by SGL (Desmons, 1998). To remove random noise, ground magnetometer data were de-spiked automatically using a filter and spiker. All ground station magnetometer data were then filtered using a 67-point lowpass filter. The airborne magnetometer data were corrected for diurnal variations in the geomagnetic field by subtracting the ground magnetometer values and adding the average value back in, using all the flights. Intersections between the control lines and the traverse lines were determined by a program that interpolates the magnetic, altitude and $\mathrm{X}$ and $\mathrm{Y}$ values of the traverse and control lines at the intersection points. Each control line was then adjusted by a specific constant value to minimize the following error for each traverse line:

$$
E|i-a|
$$

where $i$ is the individual intersection difference and $a$ is the average intersection difference for that traverse line. Individual control lines were subsequently adjusted to minimize local intersection differences, using groups of 11 adjacent traverse lines. Line leveling was carried out by a program that interpolates and extrapolates leveling values for each point, based on the two closest leveling values. Both traverse and control lines

were leveled to ensure that all intersections tied with minimum error. The International Geomagnetic Reference Field (IGRF) was removed from the leveled magnetometer data so that variations from this theoretical field were eliminated.

Because of the large spacing between control lines, the leveled magnetometer data is biased in the traverse line direction. A decorrugation (or micro-leveling) 
technique was used to improve the data. First, the leveled magnetometer data were gridded, using a minimum curvature method and a 50-meter grid spacing. Both the control and traverse lines were used to create the two-dimensional grid. SGL's decorrugation technique consists of a specialized frequency-domain filter: a low-pass filter $(500 \mathrm{~m})$ in the traverse line direction and a high-pass $(150 \mathrm{~m})$ perpendicular to the traverse line directions. The filtering procedure was implemented in the frequency domain by using a Fast-Fourier Transform (FFT). The low frequency errors from the decorrugation grid were re-sampled to the line data and subtracted from the leveled magnetometer data.

The terrain clearance measured by the radar altimeter in feet were recorded at 4 $\mathrm{Hz}$ and converted to meters. The data were filtered to remove the high-frequency noise using a 67-point filter.

The navigational data were reformatted and recalculated to get positions in differential mode. SGL's GPS data processing package, GPSoft, was used to calculate differential GPS (DGPS) positions from raw range data obtained from the airborne and ground receivers. The final location of the GPS ground station receiver was determined using a data set of corrections for selective availability from NRCan (Natural Resources Canada). This technique provided a final receiver location with an accuracy of $+/-5$ meters. The airborne data set was then reprocessed differentially using the new ground station location. The positional data were recorded using the World Geodetic System 1984 (WGS-84), which uses the geoid described by the Earth's gravitational field and a datum defined by the center of mass of the Earth (Macomber, 1984). Universal 
Transverse Mercator (UTM) coordinates were subsequently determined using the standard central meridian for Zone $12 \mathrm{~N}$. UTM is not a true map projection, but a coordinate system that divides the globe into sixty zones, each spanning six degrees of longitude. Each zone has its own central meridian from which it spans 3 degrees west and 3 degrees east (Doyle, 1997). Altitude data were also recorded in the WGS-84 datum and transformed to Mean Sea Level (MSL). 


\section{Derivative and Depth Calculations}

Derivative and depth calculations were performed on an IBM compatible PC with a $750 \mathrm{MHz}$ AMD Athlon Processor and $765 \mathrm{MB}$ of RAM running under Microsoft Windows 98. All gridding was performed using Geosoft's OASISmontaj (Geosoft, Inc., 1998). ASCII data received from Sander Geophysics Limited were imported into a Geosoft database. Positional data, originally reported in the WGS-84 datum, were converted to the NAD-27 datum in UTM coordinates with the standard central meridian for Zone $12 \mathrm{~N}$ in order to correspond with geologic and topographic maps available in that datum. The leveled magnetic anomaly data were subsequently gridded using a bidirectional line gridding algorithm (Geosoft, Inc., 1998). To allow for the smoothest grid possible, a cubic spline (Smith and Wessel, 1990) was used to interpolate grid point values that lay between the data in both the down and across-line directions. This spline worked well because of the high density of data.

All grids were displayed as shaded-relief images in order to show subtle changes in the data that may not be seen with other types of displays. Unless otherwise noted, all shaded-relief images have an azimuth sun angle of 45 degrees declination, 45 degrees inclination. Due to the simulated illumination for the shaded-relief images and the inability to use color in this report, the grey-scale legend bars do not entirely match the grey-scale images. The values and their associated shades of grey on the legend bars only match the parts of the images for which there is 100 percent illumination (i.e. surfaces facing the sun and not in the shadow of an adjacent high). The legend-bar values should only be used to get an estimate of the range of data values in the image. 
When appropriate, the amplitude range of specific anomalies, in nanoTesla, have been noted in the text. Figure 9a is a grey-scale total-field magnetic anomaly map of the entire survey area. To observe the effects that shaded relief has on a grey-scale image, Figure $9 \mathrm{~b}$ is a shaded-relief total-field magnetic-anomaly map of the same area. 


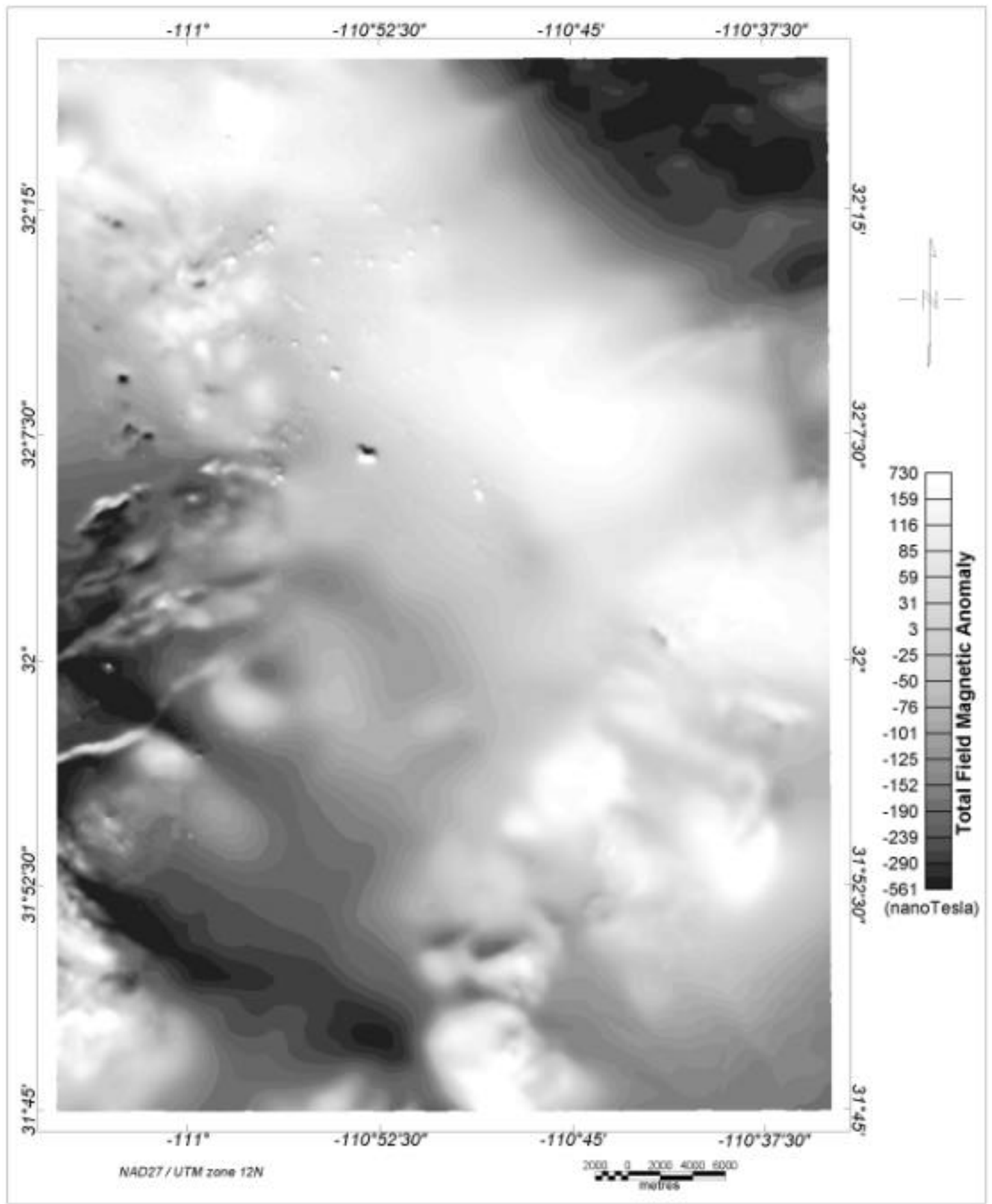

Figure 9a. Total-field magnetic magnetic anomaly map of the entire survey area. 


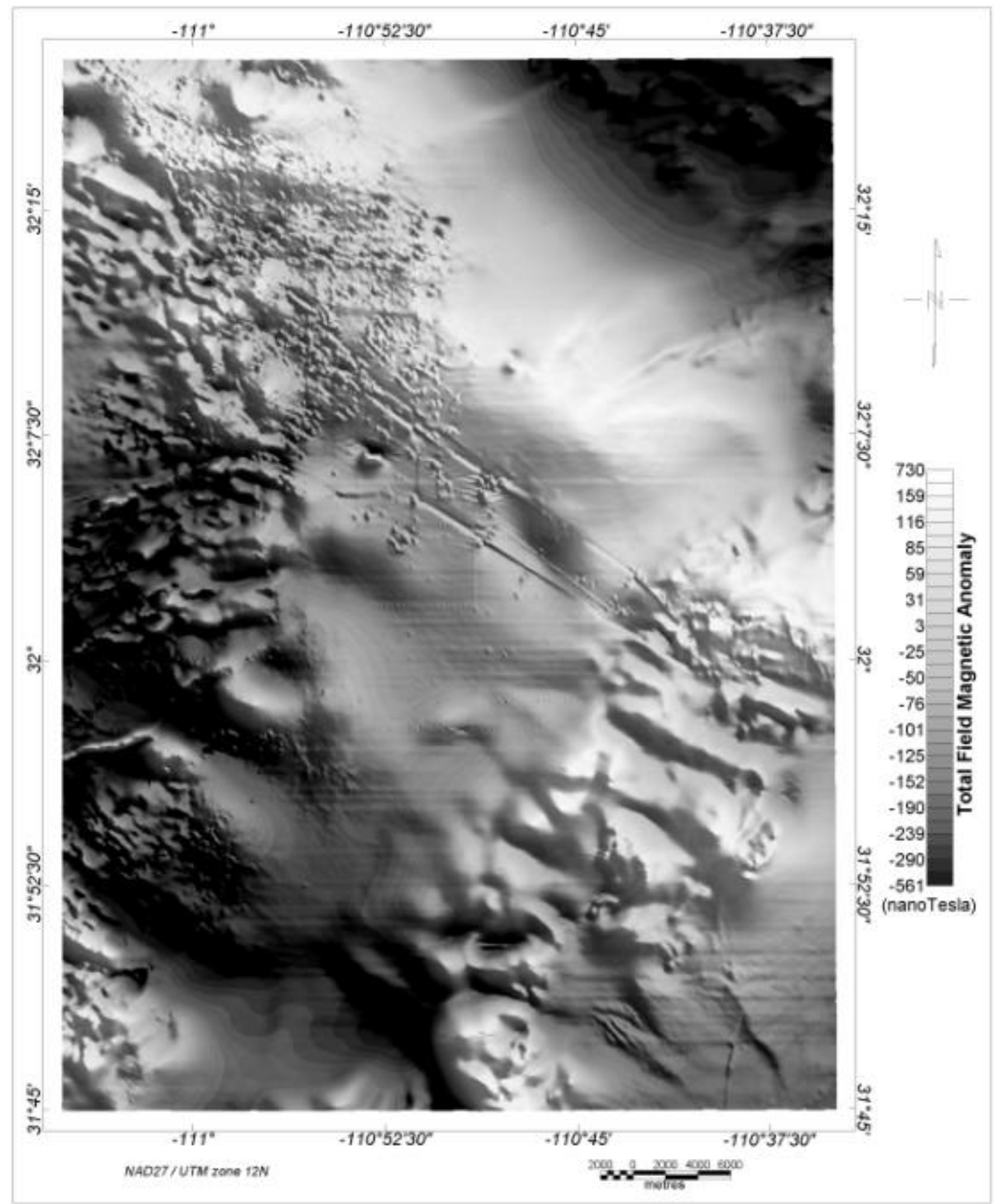

Figure 9b. Total-field magnetic magnetic anomaly map of the entire survey area. Shaded relief has been added. 
The first horizontal derivative grids, äx and äy, were calculated using a finitedifference algorithm in OASISmontaj, which passes a $3 \times 3$ data window over the grid points and finds the derivatives by subtracting grid values in the corresponding directions and taking the average. All other derivative grids, including the reduced-to-pole and pseudo-gravity grids, were calculated in the frequency domain using a Fast-Fourier Transform (FFT) algorithm that is found in OASISmontaj. The Fourier transform grids were calculated using the default settings in OASISmontaj, which included a $10 \%$ increase in grid size, the removal of a $1^{\text {st }}$ order trend based on the edge points, and maximum entropy grid filling (Burg, 1975) to match opposite sides of the grid to create a continuous signal. After the specified filters were applied to the data in the frequency domain, the data were transformed back to the spatial domain and the original grid size and $1^{\text {st }}$ order trend (if it was not removed by filtering) were restored. The signals of interest were subsequently calculated by applying mathematical expressions to the derivative grids.

Many of the depth estimates were determined using potential-field geophysical software provided by the U.S. Geological Survey (Phillips, 1997). The grids in Geosoft format were converted into USGS standard grids for use with the USGS software. Depths estimates using the simple analytic signal amplitude were calculated using ASDEP (Phillips, 1997). The analytic signal amplitude grid was used as input, and the location, strike and depth of magnetic contacts were calculated using the procedure outlined in the previous section on the simple analytic signal method. A depth-error threshold of $\leq 15 \%$ was used. Depth estimates using the horizontal gradient magnitude 
were calculated using HDEP (Phillips, 1997). This program uses a similar procedure as ASDEP and has the same output. A depth-error threshold of $\leq 15 \%$ was also used. The same approach was used for depth calculations using the local wavenumber. Here, the program LOCDEP (Phillips, 1997) was used, and a depth-error threshold of $\leq 15 \%$ was also used.

Output from ASDEP, HDEP and LOCDEP is in the form of a USGS post file that includes $x$ and $y$ locations, calculated depths, calculated depth errors and strikes. The post files were imported nto an OASISmontaj database. Strike data were converted into vector information for the purpose of plotting. The vector information was used to plot headless arrows on a map with their centers at the calculated locations. The headless arrows were used to display the calculated strikes appropriately. All preserved depth solutions were then plotted in plan view, using symbols that were proportional in size to the depths (Appendices A, B and C). Solutions not of interest (i.e. those solutions not over the contacts of interest) were masked out by creating a polygon mask within OASISmontaj and applying the mask to the data.

Depth estimates for the enhanced analytic signal and the enhanced local wavenumber were not calculated using the USGS potential-field software. However, the locations and strikes of the magnetic contacts were determined by inputting the second order analytic signal amplitude grid and the first order local wavenumber grid into ASDEP and LOCDEP, respectively. A depth-error threshold of $\leq 50 \%$ for the second order analytic signal magnitude grid was used instead of $\leq 15 \%$ in order to acquire a significant number of solutions. The second order analytic signal amplitude does not 
have the same shape as the simple analytic signal amplitude over magnetic contacts (see Equations 24 and 29). Therefore, the calculated error is not indicative of the reliability of the depth solution, and a threshold of $\leq 15 \%$ does not yield a sufficient number of solutions. A threshold of $\leq 15 \%$, however, was used for the second order local wavenumber. The higher order local wavenumber differs only in amplitude and not in shape from the first order analytic signal (Smith et al., 1998). Depth estimates for the enhanced analytic signal method were calculated by sampling the lower order analytic signal amplitude grids at the locations of the second-order analytic signal amplitude peaks and then using the appropriate equations to solve for depth. For coordinates that did not correspond to exact grid point locations, the data values were interpolated using a linear interpolation. Depth estimates for the enhanced local wavenumber method were calculated by sampling the simple local wavenumber at the locations of enhanced local wavenumber peaks and using Equation 42 to solve for depth. The strikes and depths were plotted in the same manner as in the other methods.

Euler depth estimates were calculated using the Euler deconvolution algorithm that is included in OASISmontaj (Geosoft, Inc., 1998). A data-window size and structural index were chosen based on the size of the anomaly and geometry of the causative source, respectively. A depth-error threshold of $\leq 15 \%$ was used. All preserved solutions were then plotted in plan view, using symbols that were proportional to the depths.

For the three test sites that were used in this study, the flight elevations were assumed equal to the depth to the magnetic contacts. The radar altimeter data were 
gridded at 50 meters using the same bi-directional gridding algorithm that was used for the magnetometer data. At the calculated depth coordinates, the radar altimeter grid was sampled to provide an approximated flight elevation. The sampled radar altimeter data were subsequently subtracted from the depth estimates. The resulting values were used to provide a depth-to-source error for each calculated depth estimate using the following equation:

$$
\text { Error }=\frac{\text { CalculatedDepth }- \text { FlightElevation }}{\text { FlightElevation }}
$$

Negative errors represent depth estimates that are too shallow. Positive errors represent depth estimates that are too deep.

To see how preprocessing of the data would affect depth calculations, calculations were performed over the test sites using high-pass filtered magnetic anomaly data as well as the total-field magnetic anomaly data. Applying a high-pass filter to the total-field anomaly data was done to remove deeper-source magnetic anomalies that may be interfering with the anomaly of interest. A high-pass Butterworth filter with a cutoff wavelength of 2500 meters/cycle was applied to the gridded total-field magnetic anomaly data. This particular filter allows the user to control the degree of filter roll-off by inputting a constant that defines the degree of the function. An $8^{\text {th }}$ degree function was used for the high-pass filter. These filter parameters removed most of the deeper-source signal amplitudes while preserving the entire signal of the anomalies of interest in this study. 
In addition to calculating depth estimates using high-pass filtered data, depth estimates were calculated using pseudo-gravity anomaly data, of both the total-field and residual-field magnetic anomaly data, to compare maximum with minimum depths using the horizontal gradient and simple and enhanced analytic signal methods. A declination of 11.8 degrees, an inclination of 59.2 degrees, a density contrast of $1 \mathrm{~g} / \mathrm{cc}$ and a magnetization of 0.5 gauss $(50,000 \mathrm{nT})$ were used as the input parameters for the pseudogravity transform filter. 


\section{TEST SITES}

\section{Los Reales Landfill}

The Los Reales Landfill, located in southeastern Tucson (Figure 10), is a public landfill operated by the City of Tucson. It is nearly 1100 by 800 meters in aerial extent and is continuing to grow. Its topographic expression is one of a large semi-rectangular flat-topped mound, with a height of more than 20 meters (Figure 11). Surrounding the central mound are areas of excavation that are nearly 20 meters deep. These are sites of future dumping, which will presumably be filled up to the height of the central mound and covered over with fill dirt. A vertical profile through the center of the landfill would reveal a football-shaped geometry, fat in the middle and pinching out at the ends.

The landfill is relatively isolated from any other shallow magnetic sources, since it is located away from the center of town and associated cultural noise. The magnetic expression of the landfill is one of a nearly perfect dipole with a declination around 12 degrees (i.e. the same as the local reference field). The magnetic field is considered to be induced with little or no remanent magnetization and has a total range in amplitude of nearly $824 \mathrm{nT}$, peak to trough (Figure 12). The field anomaly is the result of a random distribution of ferrous materials buried within the landfill. Random orientations of these ferrous materials result in an overall cancellation of any remanent magnetizations that may exist, and only the induced components of the total magnetization vectors remain. This distribution of ferrous materials can be considered similar to a distribution of ferric minerals found in a geologic body such as an ore 


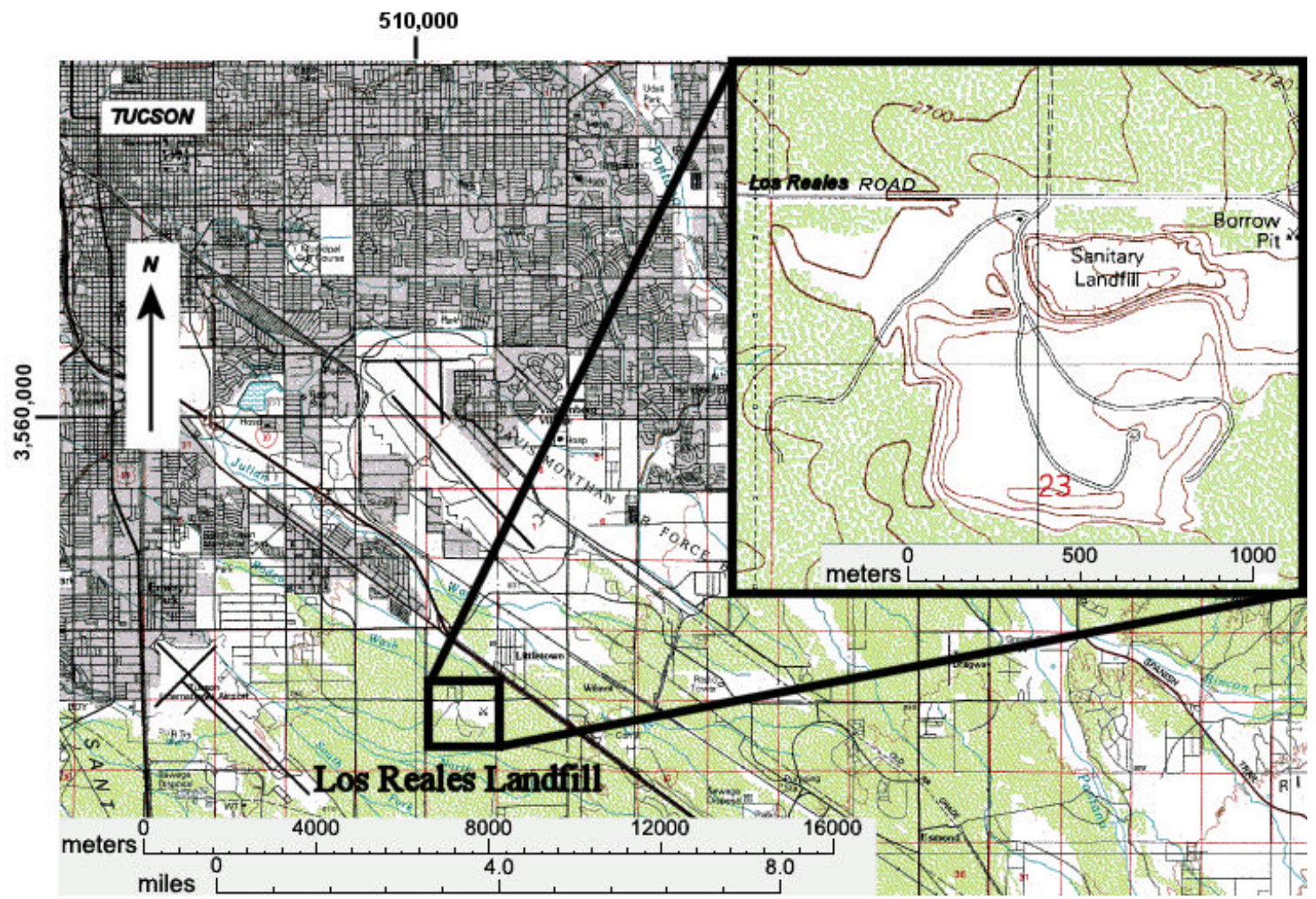

Figure 10. Location of the Los Reales Landfill in southeastern Tucson (modified from TopoZone, 2000). 


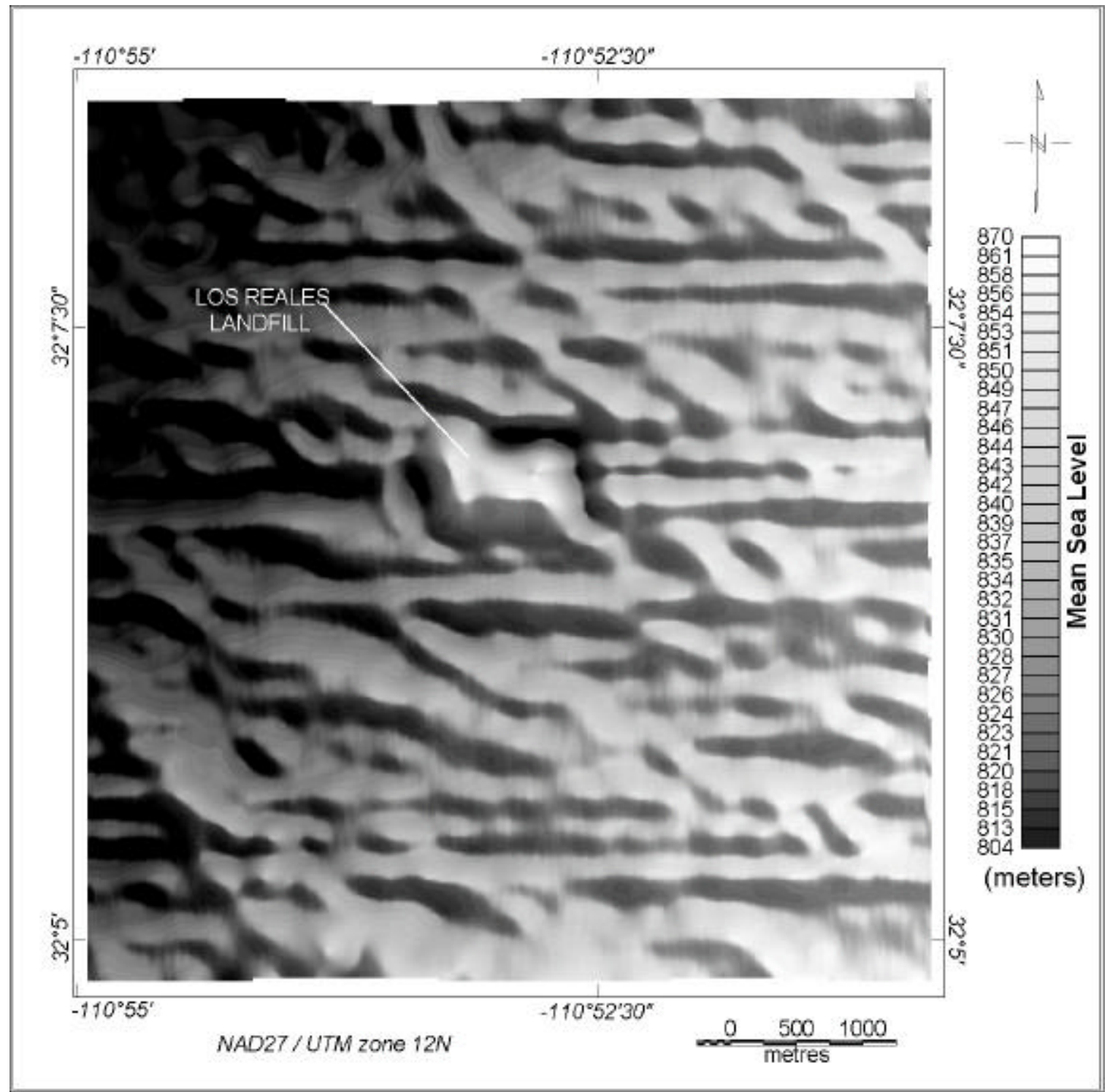

Figure 11. Topographic map created from radar altimeter and elevation data collected during the survey over the Los Reales Landfill. 


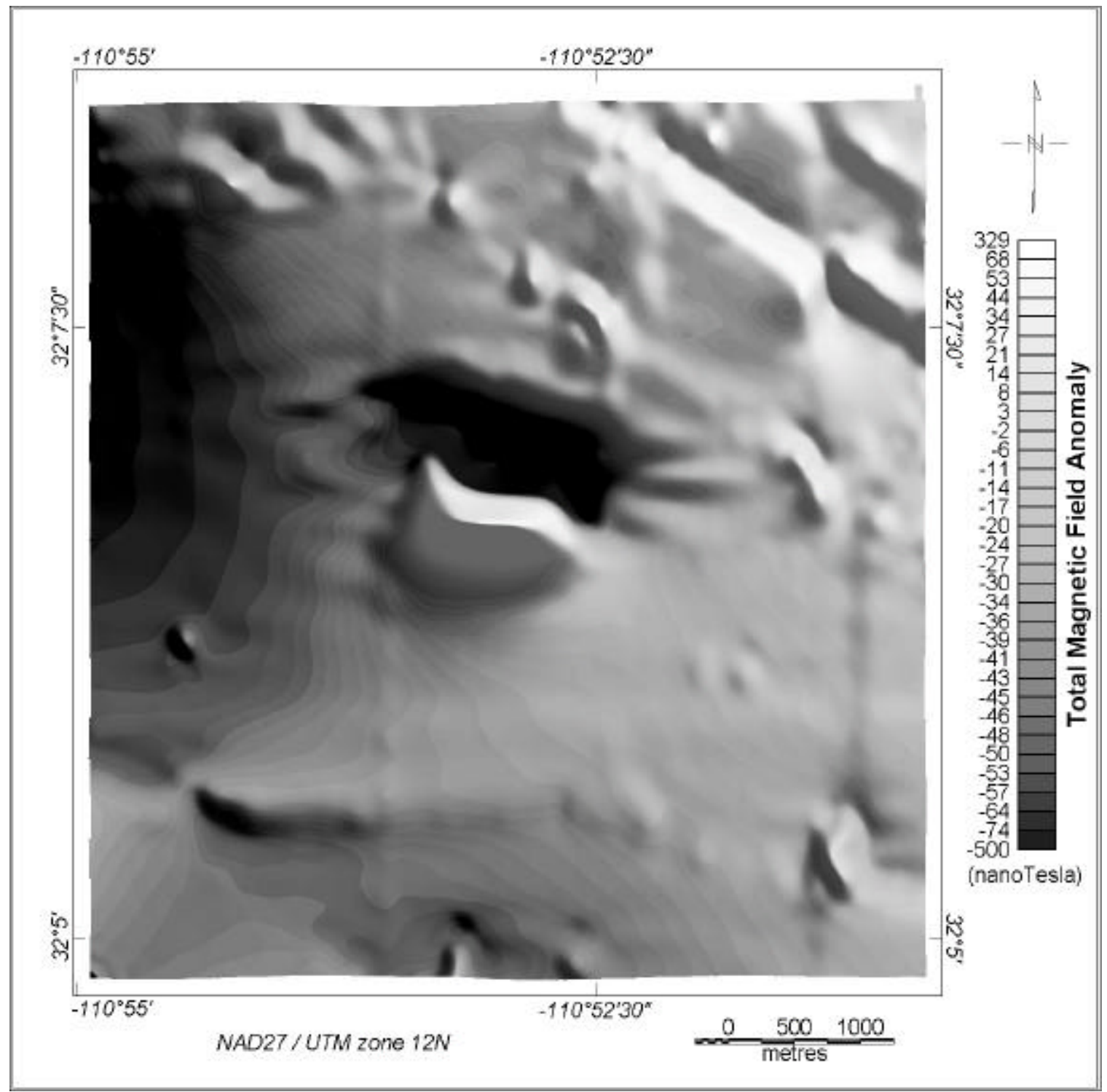

Figure 12. Total magnetic field anomaly map over the Los Reales Landfill.

deposit. This makes the landfill a perfect target for testing depth-to-source calculations on a shallow source with a purely induced field.

The magnetic contact locations were considered to be where the landfill pinches out at its edges. These contacts surround the semi-rectangular mound structure and are effectively at ground level. All of the methods discussed in this study were used to 
calculate the depth to the magnetic contacts around the landfill, and a depth-to-source error was calculated using the flight height as the correct solution.

The reduced magnetometer data were gridded with a 5-meter grid interval using the bi-directional line gridding algorithm in OASISmontaj. Before any derivatives were calculated, a 150-meter low-pass Butterworth filter, using an $8^{\text {th }}$ degree function, was applied to the gridded data. All signals were subsequently calculated from the low-pass filtered 5-meter grid. The grids were re-gridded at a larger interval before any depths were calculated. Before re-gridding, a low-pass linear convolution filter with a length of the new grid spacing was used in the direction of the grid lines. For all but the local wavenumber methods, a 50-meter grid interval was used to find magnetic contacts. For the local wavenumber methods, a 20-meter grid interval was used.

A structural index of 1 was used for Euler deconvolution, since the edges of the landfill have effectively two infinite dimensions and approximate the edges of a sill. The search window size was set to 750 meters with a maximum search radius of 375 meters, which windowed enough of the anomaly to calculate the depth without including interfering anomalies. For the enhanced analytic signal, the formula for an infinite step (Equation 32) was used to calculate the depth. Table 1 summarizes the depth-to-source error results for each method. Appendix A contains the maps of the specific signals used for each calculation with the relevant calculated strikes and depths plotted as lines and circles, respectively. 
Table 1. Depth-to-source error statistics for the Los Reales Landfill.

$\%$

\begin{tabular}{l|ccccccccc} 
Method & Median & Mean & Standard Dev. & Kurtosis & Skewness & Range & Minimum & Maximum & Count \\
\hline euler & 6.1 & 7.6 & 35.9 & 0.5 & 0.5 & 240.0 & -100.0 & 140.0 & 2261 \\
hpeuler & 10.2 & 10.1 & 19.4 & -0.3 & 0.0 & 110.4 & -48.3 & 62.1 & 798 \\
hg & -30.6 & -29.8 & 8.1 & -0.6 & 0.1 & 32.3 & -45.4 & -13.1 & 24 \\
hphg & -32.4 & -31.3 & 6.7 & 0.4 & 0.7 & 29.0 & -42.3 & -13.3 & 27 \\
sas & -1.9 & 0.5 & 11.8 & 0.1 & 0.8 & 42.6 & -13.5 & 29.1 & 20 \\
hpsas & -5.9 & -4.5 & 14.9 & 0.7 & 0.0 & 61.7 & -36.0 & 25.7 & 18 \\
eas & -10.4 & -14.3 & 29.7 & 16.2 & 2.9 & 207.2 & -65.7 & 141.5 & 47 \\
hpeas & -11.1 & -17.0 & 19.5 & 1.9 & 0.0 & 114.0 & -65.0 & 49.1 & 52 \\
lwn & -33.1 & -36.2 & 20.1 & -1.1 & -0.3 & 71.8 & -80.2 & -8.4 & 57 \\
hplwn & -39.3 & -42.5 & 21.0 & -0.8 & 0.0 & 82.9 & -81.3 & 1.6 & 53 \\
vilwn & -3.4 & -1.8 & 28.3 & -1.2 & -0.1 & 94.6 & -51.6 & 43.0 & 43 \\
hpvilwn & -1.3 & -1.0 & 28.2 & -1.5 & 0.2 & 84.6 & -39.0 & 45.5 & 44 \\
elwn & 41.2 & 41.6 & 63.1 & 1.8 & 0.4 & 321.2 & -91.1 & 230.0 & 36 \\
hpelwn & 49.7 & -11.3 & 146.9 & 3.6 & -2.2 & 572.5 & -490.8 & 81.7 & 46 \\
hgpg & 11.2 & 12.3 & 18.7 & -0.8 & 0.3 & 68.8 & -14.8 & 54.1 & 44 \\
hphgpg & 5.0 & 6.0 & 15.7 & 0.0 & 0.5 & 69.7 & -25.9 & 43.9 & 44 \\
saspg & 45.7 & 63.0 & 27.6 & 2.7 & 1.7 & 81.4 & 43.8 & 125.2 & 9 \\
hpsaspg & 53.2 & 65.1 & 41.7 & 1.9 & 1.1 & 131.7 & 12.6 & 144.3 & 7 \\
easpg & 36.1 & 36.0 & 10.0 & -0.4 & -0.3 & 39.3 & 12.5 & 51.8 & 38 \\
hpeaspg & 25.0 & 27.7 & 17.2 & 2.1 & 1.1 & 90.5 & -7.9 & 82.6 & 41
\end{tabular}

euler - euler deconvolution

hg - horizontal gradient

hp - calculated from high-pass filtered data

pg - calculated from pseudo-gravity data

sas - simple analytic signal

eas - enhanced analytic signal

vi - calculated from the vertical integral

Iwn - local wavenumber

elwn- enhanced local wavenumber

Figure 13 shows the depth-to-source error histograms for all of the methods used. The histogram for Euler deconvolution reveals that most of the depth estimates (56\%) are too deep. Nearly two-thirds of the total number of depth estimates, many of which occur in a "tail" in the error distribution towards deeper solutions, were eliminated when a high-pass filter was applied to the magnetic anomaly data before calculating depth estimates. The high-pass filter resulted in a distribution that is closer to a normal distribution, but still with most of the depth estimates (69\%) occurring too deep. All of the depth estimates from the horizontal gradient and local wavenumber methods, when 
applied to the total-field magnetic anomaly data, are too shallow, with no depth estimates occurring on the positive side of the error distributions. Filtering seems to have had little effect on the number and distribution of depth estimates, apart from the removal of a few deeper estimates. For the simple analytic signal method, most of the depth estimates (55\%) are too shallow, but close to the true depth to source. A high-pass filter introduced some shallower solutions and removed some deeper solutions, which caused the error distribution to be more evenly distributed about a common value and have a majority of depth estimates $(56 \%)$ occurring too deep. The enhanced analytic signal method resulted in most of the depth estimates (89\%) occurring too shallow. Filtering had little effect, except for the removal of a single deep solution that was far from the main distribution of error values. Most of the depth estimates (56\%) from the local wavenumber method applied to the vertical integral of the total-field magnetic anomaly data are too shallow, but distributed fairly evenly about a common value close to the true depth to source. Applying a high-pass filter before calculating depth estimates resulted in the removal of shallower depth estimates and introduced a few deeper estimates, resulting in a nearly even distribution between depth estimates that are too deep and depth estimates that are too shallow (48\% vs. 52\%, respectively). For the enhanced local wavenumber method, the error distributions are very sporadic. Most of the solutions (83\%) are too deep, with a few having errors greater than $100 \%$. Applying a high-pass filter resulted in the removal of many deep solutions. However, filtering also introduced a few shallow solutions with error values less than $-100 \%$. The resulting error distribution still has most of the depth estimates (74\%) occurring too deep. The 
horizontal gradient method applied to the pseudo-gravity anomaly field resulted in most of the depth estimates (73\%) occurring too deep. Applying a high-pass filter to the data removed many of the deeper solutions and introduced more shallow solutions, resulting in depth estimates clustering around a value closer to the true depth to source, but still with most of the depth estimates (59\%) occurring too deep. The simple and enhanced analytic signal methods applied to the pseudo-gravity anomaly field yielded solutions that are much too deep, with no depth estimates occurring on the negative side of the error distributions. A high-pass filter introduced both shallower and deeper solutions. For all of the depth-to-source methods combined, most of the depth estimate (63\%) are too deep, and the median depth-to-source error is around six percent. 
Figure 13. Depth-to-source error histograms for each method over the Los Reales Landfill.
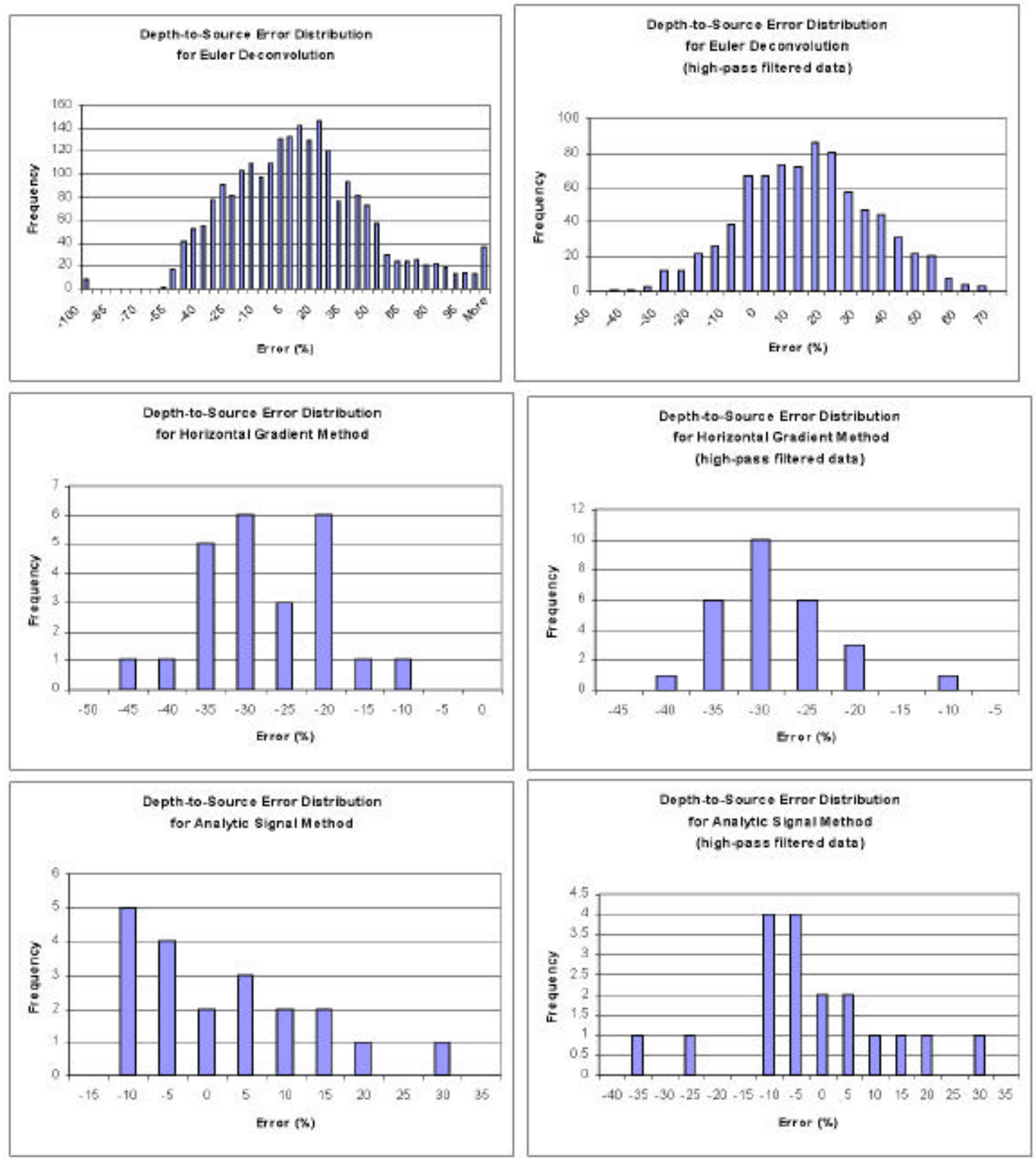
Figure 13, cont...
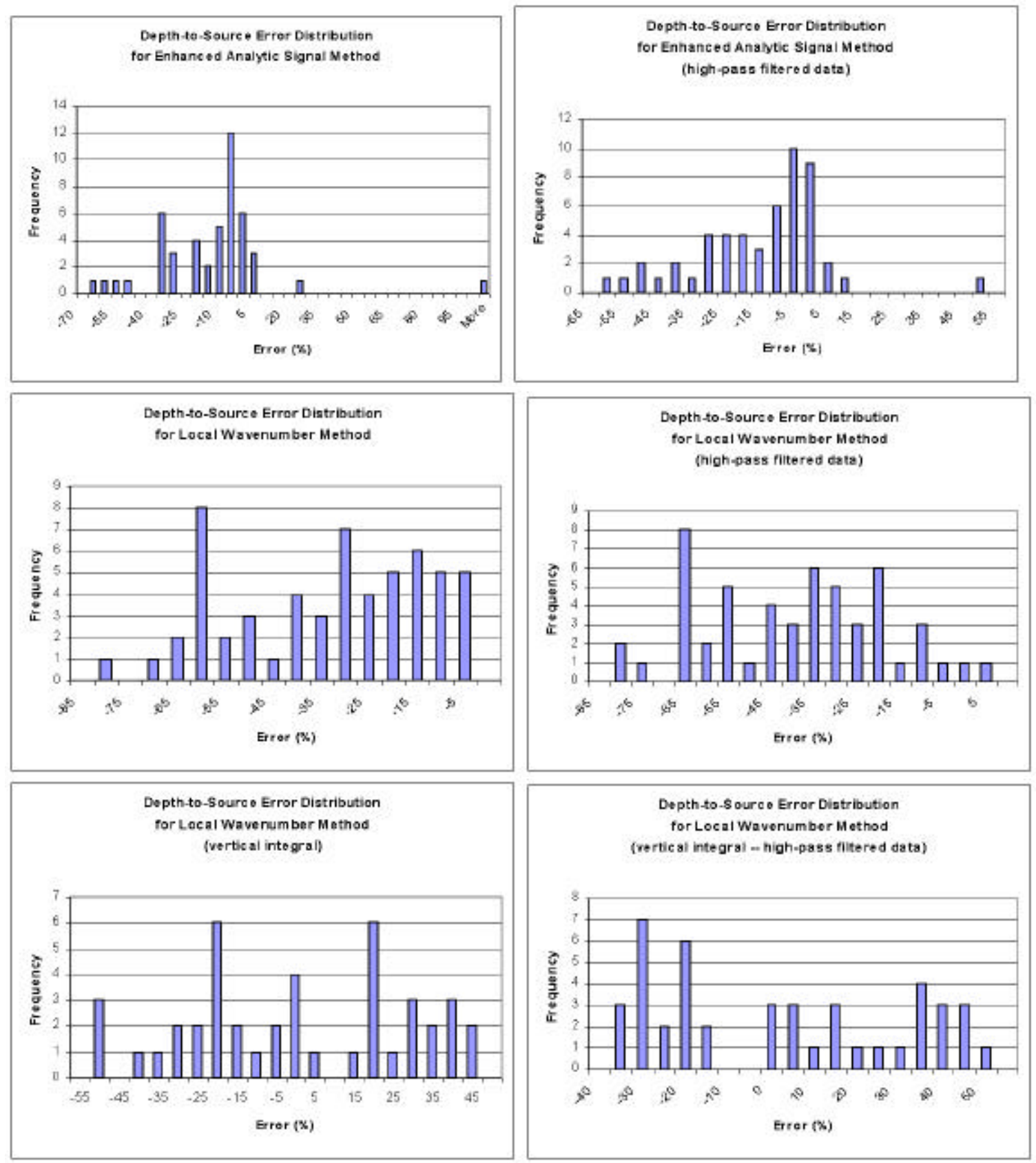
Figure 13, cont...
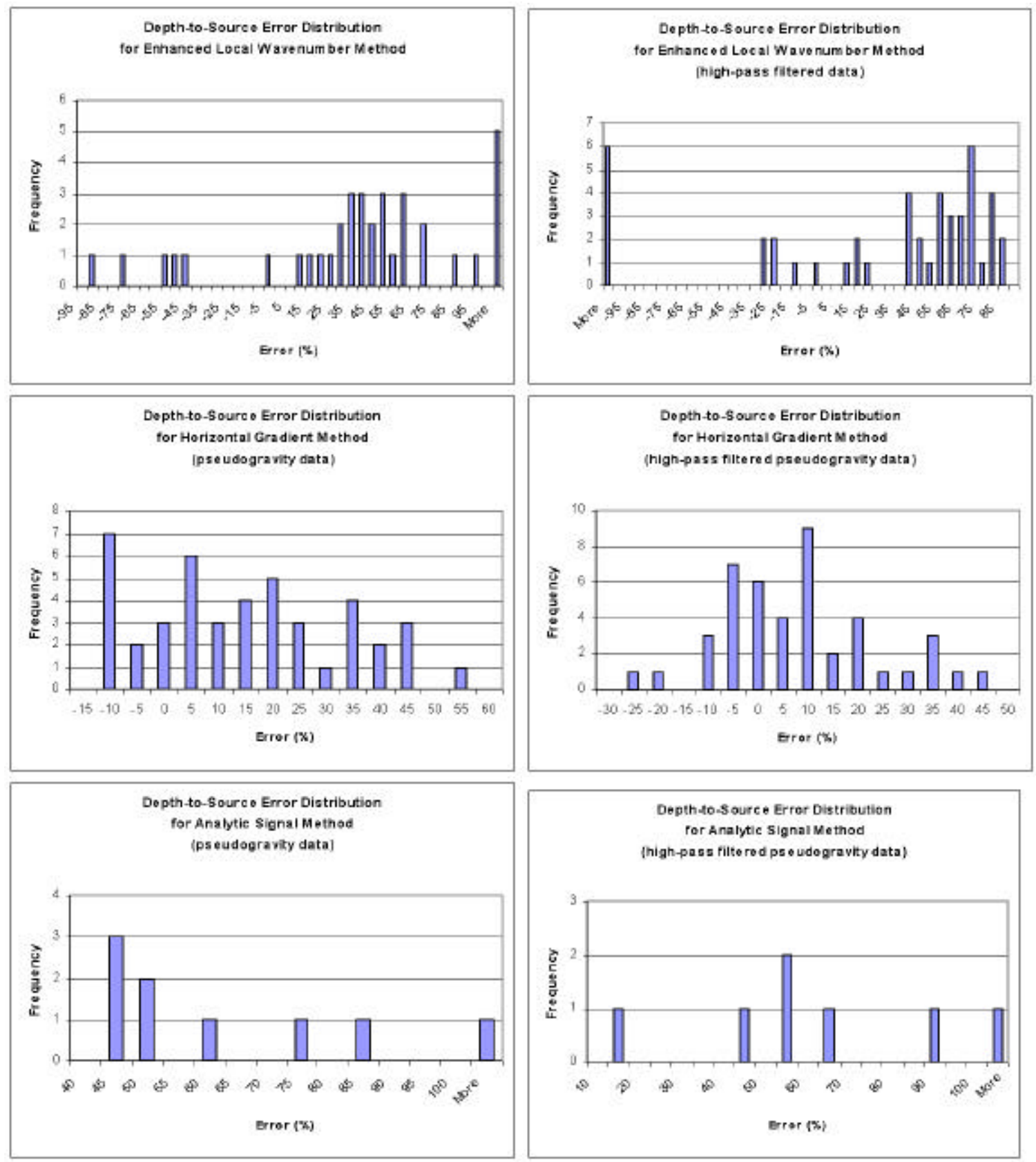
Figure 13, cont...
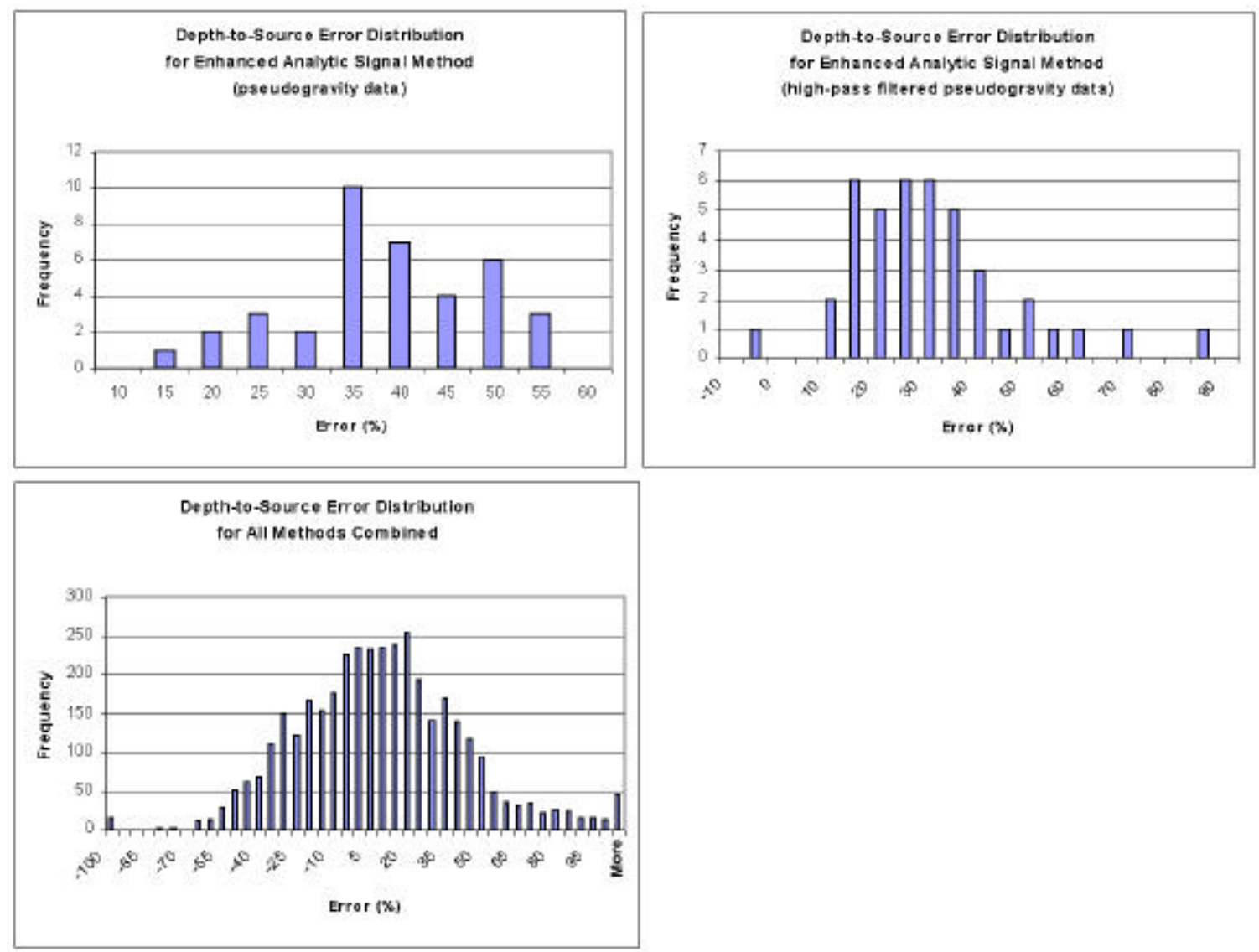


\section{Black Mountain}

Black Mountain, located southwest of Tucson on the San Xavier Indian Reservation (Figure 14), is made up of a series of Tertiary volcanic sequences (Middle Miocene to Oligocene) that are primarily flat-lying to gently-dipping flows of basalt and basaltic andesite, with interbedded sedimentary rocks and tuff (Drewes, 1980). It is over eight square-kilometers in aerial extent. Black Mountain's topographic expression peaks at a height of nearly 290 meters above the valley floor (Figure 15). (Note, all shaded relief images of the Black Mountain area have an azimuth sun angle of 315 degrees declination, 45 degrees inclination).

Black Mountain is located near the edge of the Tucson basin and away from the center of town and associated cultural noise. Its magnetic expression is one of a reversed dipole, with a declination around 150 degrees. The area is dominated by strong remanent magnetization and has a total range in amplitude of nearly $534 \mathrm{nT}$, peak to trough (Figure 16). The remanent field is the result of magnetite grains that formed while lava cooled in the presence of a geomagnetic field having a different orientation than today's field. The reversed anomaly of Black Mountain makes a perfect target for testing depth-to-source calculations on a shallow source with strong remanent magnetization.

The magnetic contact locations were considered to be where the volcanic sequences are truncated by the valley fill and where multiple flows possessing different remanent magnetizations are in contact. These contacts are found within and surrounding the topographic expression of Black Mountain and are effectively at ground level. All of the methods discussed in this study were used to calculate the depth to the magnetic 
contacts around Black Mountain, and a depth-to-source error was calculated using the flight height as the correct solution.

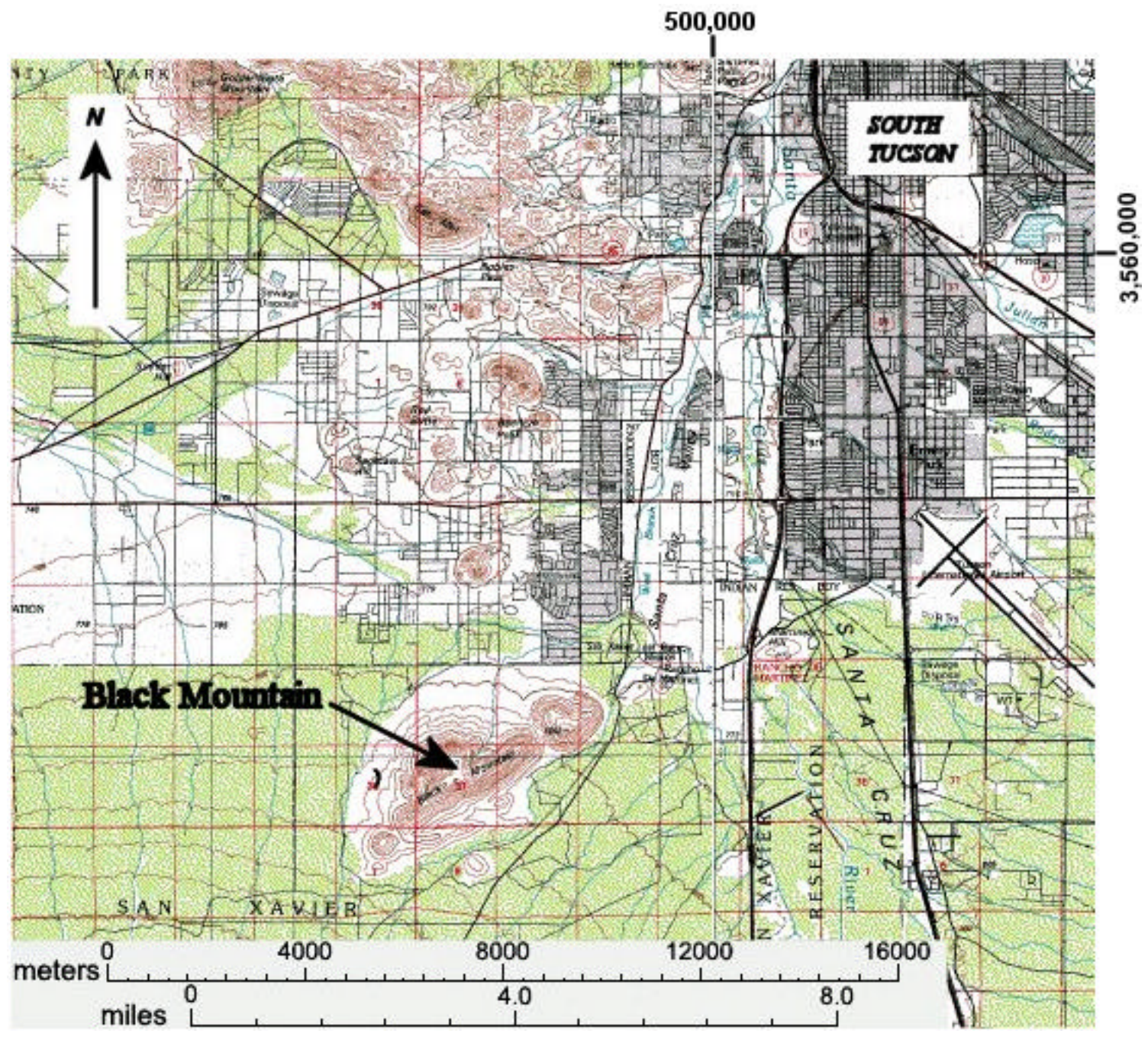

Figure 14. Location of Black Mountain southwest of Tucson (modified from TopoZone, 2000). 


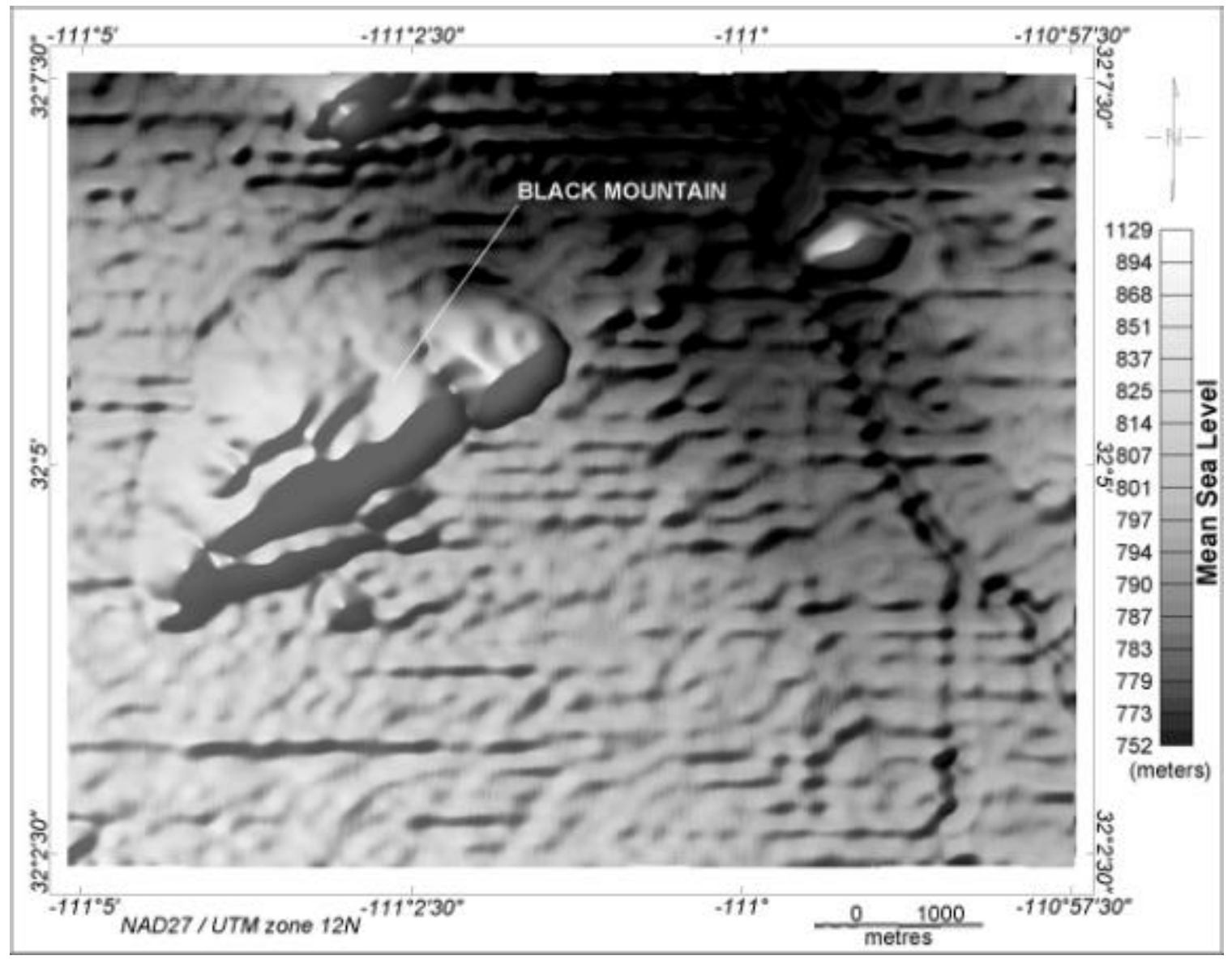

Figure 15. Topographic map created from radar altimeter and elevation data collected during the survey over Black Mountain. 


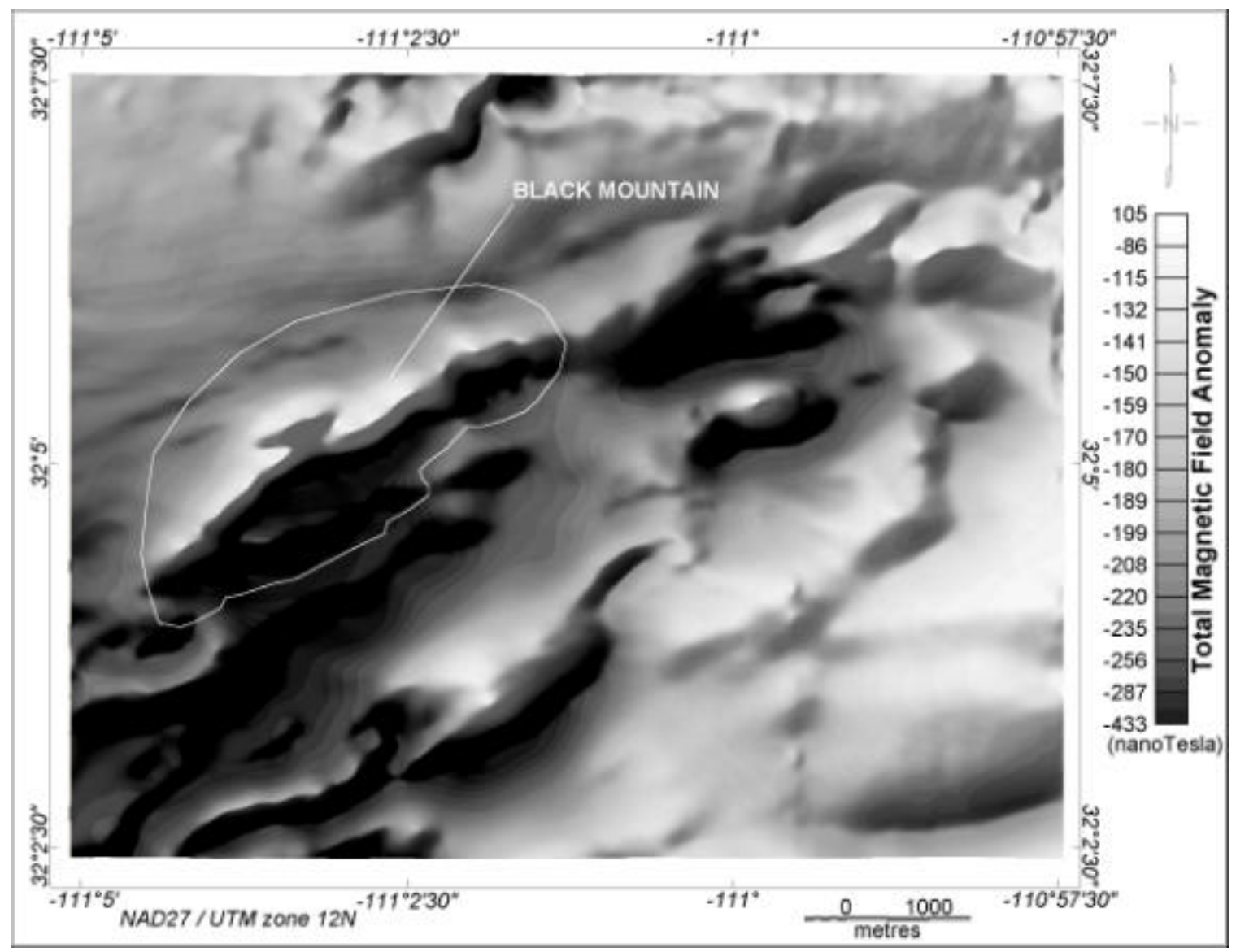

Figure 16. Total magnetic field anomaly map over Black Mountain. The white outline borders the topographic expression of Black Mountain.

The reduced magnetometer data were gridded with a 10-meter grid interval using the bi-directional line gridding algorithm in OASISmontaj. Before any derivatives were calculated, a 150-meter low-pass Butterworth filter, using an $8^{\text {th }}$ degree function, was applied to the gridded data. All signals were subsequently calculated from the lowpass filtered 10-meter grid. The grids were re-gridded at a larger interval before any depths were calculated. Before re-gridding, a low-pass linear convolution filter with a length of the new grid spacing was used in the direction of the grid lines. For all but the 
local wavenumber methods, a 50-meter grid interval was used to find magnetic contacts. For the local wavenumber methods, a 20-meter grid interval was used.

The edges of Black Mountain have effectively two infinite dimensions but are suspected to be thicker than the edges of the landfill. A structural index of 1 and 0.5 were used for Euler deconvolution to compare solution clustering and compare depth estimates for different structural indices. The search window size was set to 750 meters with a maximum search radius of 375 meters, which windowed enough of the anomaly to calculate the depth without including interfering anomalies. For the enhanced analytic signal, the formula for an infinite step (Equation 32) was used to calculate the depth. Table 2 summarizes the depth-to-source error results for each method. Appendix B contains the maps of the specific signals used for each calculation with the relevant calculated strikes and depths plotted as lines and circles, respectively. 
Table 2. Depth-to-source error statistics for Black Mountain.

\begin{tabular}{|c|c|c|c|c|c|c|c|c|c|}
\hline Method & Median & Mean & Standard Dev. & Kurtosis & Skewness & Range & Minimum & Maximum & Count \\
\hline euler $N=1$ & 27.2 & 31.7 & 27.8 & 0.2 & 0.9 & 135.2 & -19.8 & 115.4 & 1010 \\
\hline hpeuler $N=1$ & 26.2 & 29.5 & 26.3 & 0.0 & 0.8 & 127.0 & -16.4 & 110.6 & 1001 \\
\hline euler $N=0.5$ & -20.4 & -14.9 & 22.0 & 1.2 & 1.2 & 125.4 & -57.0 & 68.4 & 1115 \\
\hline hpeuler $N=0.5$ & -20.5 & -14.8 & 21.5 & 1.1 & 1.1 & 117.0 & -50.2 & 66.8 & 1077 \\
\hline$h g$ & -33.5 & -30.8 & 20.0 & 0.6 & 0.8 & 101.8 & -68.2 & 33.6 & 91 \\
\hline hphg & -32.8 & -32.1 & 18.6 & 0.6 & 0.7 & 96.5 & -65.7 & 30.8 & 113 \\
\hline sas & 10.5 & 12.9 & 22.1 & 0.3 & 0.9 & 92.8 & -18.3 & 74.4 & 35 \\
\hline hpsas & 8.1 & 12.3 & 25.4 & 1.0 & 0.4 & 131.4 & -51.3 & 80.1 & 37 \\
\hline eas & -12.7 & -13.5 & 19.4 & -0.3 & 0.1 & 87.3 & -49.0 & 38.2 & 67 \\
\hline hpeas & -13.0 & -14.0 & 18.4 & -0.7 & -0.1 & 72.6 & -51.4 & 21.2 & 78 \\
\hline Iwn & -37.4 & -37.1 & 30.7 & -0.3 & 0.3 & 164.9 & -89.4 & 75.5 & 190 \\
\hline hplwn & -48.4 & -40.9 & 31.0 & -0.1 & 0.6 & 160.7 & -88.0 & 72.7 & 217 \\
\hline vilwn & -32.7 & -25.5 & 34.9 & -1.0 & 0.4 & 145.2 & -86.1 & 59.1 & 151 \\
\hline hpvilwn & -10.7 & -13.1 & 36.0 & -1.0 & -0.2 & 137.3 & -85.6 & 51.7 & 128 \\
\hline elwn & -6.5 & 29.2 & 257.2 & 230.9 & 14.6 & 4310.9 & -200.5 & 4110.5 & 278 \\
\hline hpen/wn & 25.8 & 38.6 & 97.5 & 68.8 & 6.9 & 1099.6 & -69.6 & 1030.0 & 156 \\
\hline hgpg & 12.5 & 14.3 & 39.8 & 1.6 & 0.8 & 225.5 & -65.8 & 159.7 & 111 \\
\hline hphgpg & -0.1 & 4.6 & 38.9 & 2.1 & 1.2 & 216.0 & -60.4 & 155.7 & \\
\hline saspg & 68.7 & 71.1 & 25.0 & 0.4 & -0.2 & 117.3 & 3.2 & 120.5 & 47 \\
\hline hpsaspg & 75.3 & 76.7 & 23.9 & -0.6 & -0.2 & 99.5 & 18.6 & 118.2 & 42 \\
\hline easpg & 43.7 & 40.8 & 16.8 & -0.6 & -0.3 & 61.4 & 7.6 & 69.0 & 16 \\
\hline hpeaspg & 40.0 & 35.4 & 18.1 & 0.4 & -0.9 & 70.8 & -9.0 & 61.8 & 21 \\
\hline \multicolumn{4}{|c|}{ euler - euler deconvolution } & \multicolumn{6}{|c|}{ hp - calculated from high-pass filtered data } \\
\hline \multirow{2}{*}{\multicolumn{4}{|c|}{$\begin{array}{ll}\text { hg } & \text { - horizontal gradient } \\
\text { sas } & \text { - simple analytic signal }\end{array}$}} & \multicolumn{6}{|c|}{ pg - calculated from pseudo-gravity data } \\
\hline & & & & \multicolumn{6}{|c|}{ - calculated from the vertical integral } \\
\hline \multicolumn{4}{|c|}{ eas - enhanced analytic signal } & - stru & ctural index & & & & \\
\hline
\end{tabular}

Figure 17 shows the depth-to-source error histograms for all of the methods used. The histogram for Euler deconvolution using a structural index of 1 reveal that most of the depth estimates (92\%) are too deep. For an index of 0.5 , most of the depth estimates (80\%) are too shallow. Applying a high-pass filter before calculating depth estimates seems to have had little effect on the number and distribution of depth estimates for both indices. Most of the depth estimates from the horizontal gradient (91\%) and local wavenumber $(91 \%)$ methods are too shallow. Filtering introduced more shallow depth estimates for the local wavenumber method and did not seem to have much of an 
effect on the results from the horizontal gradient method. For the simple analytic signal method, most of the depth estimates $(71 \%)$ are too deep, but close to the true depth to source. A high-pass filter did not seem to affect the results very much, apart from the introduction of a single, very shallow depth estimate. The enhanced analytic signal method resulted in most of the depth estimates (76\%) occurring too shallow. Filtering only removed a few deeper solutions. Most of the depth estimates (72\%) from the local wavenumber method applied to the vertical integral of the total-field magnetic anomaly data are too shallow. Applying a high-pass filter before calculating depth estimates resulted in a greater number of deeper depth estimates that caused a fairly even distribution about a common value close to the true depth to source. The majority of depth estimates (60\%), however, are still too shallow. For the enhanced local wavenumber method, the error distributions are very sporadic. Most of the depth estimates (53\%) are too shallow, and many solutions have errors greater than $100 \%$. Applying a high-pass filter resulted in the removal of many shallow depth estimates, which resulted in most of the solutions (61\%) occurring too deep. The horizontal gradient method applied to the total-field pseudo-gravity anomaly data resulted in most of the depth estimates (63\%) occurring too deep. Applying a high-pass filter to the data removed many of the deeper solutions and introduced more shallow solutions, resulting in depth estimates clustering around a value closer to the true depth to source, but still with most of them (51\%) occurring too shallow. The simple and enhanced analytic signal methods applied to the total-field pseudo-gravity anomaly data yielded solutions that are much too deep, with no depth estimates occurring on the negative side of the 
error distributions. A high-pass filter introduced both shallower and deeper solutions. For all of the depth-to-source methods combined, most of the depth estimate (53\%) are too deep, and the median depth-to-source error is around three percent. 
Figure 17. Depth-to-source error histograms for each method over Black Mountain.
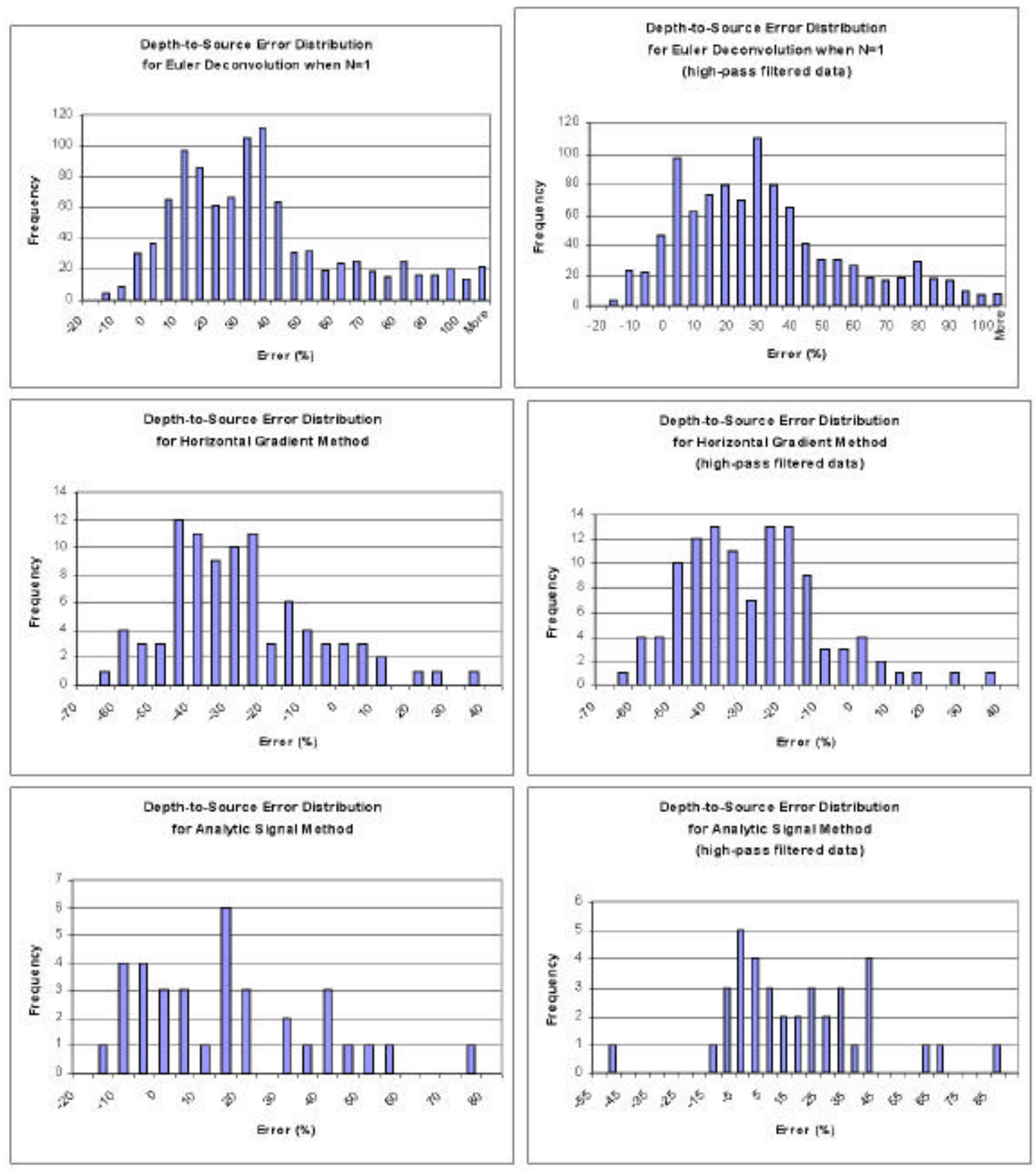
Figure 17, cont...
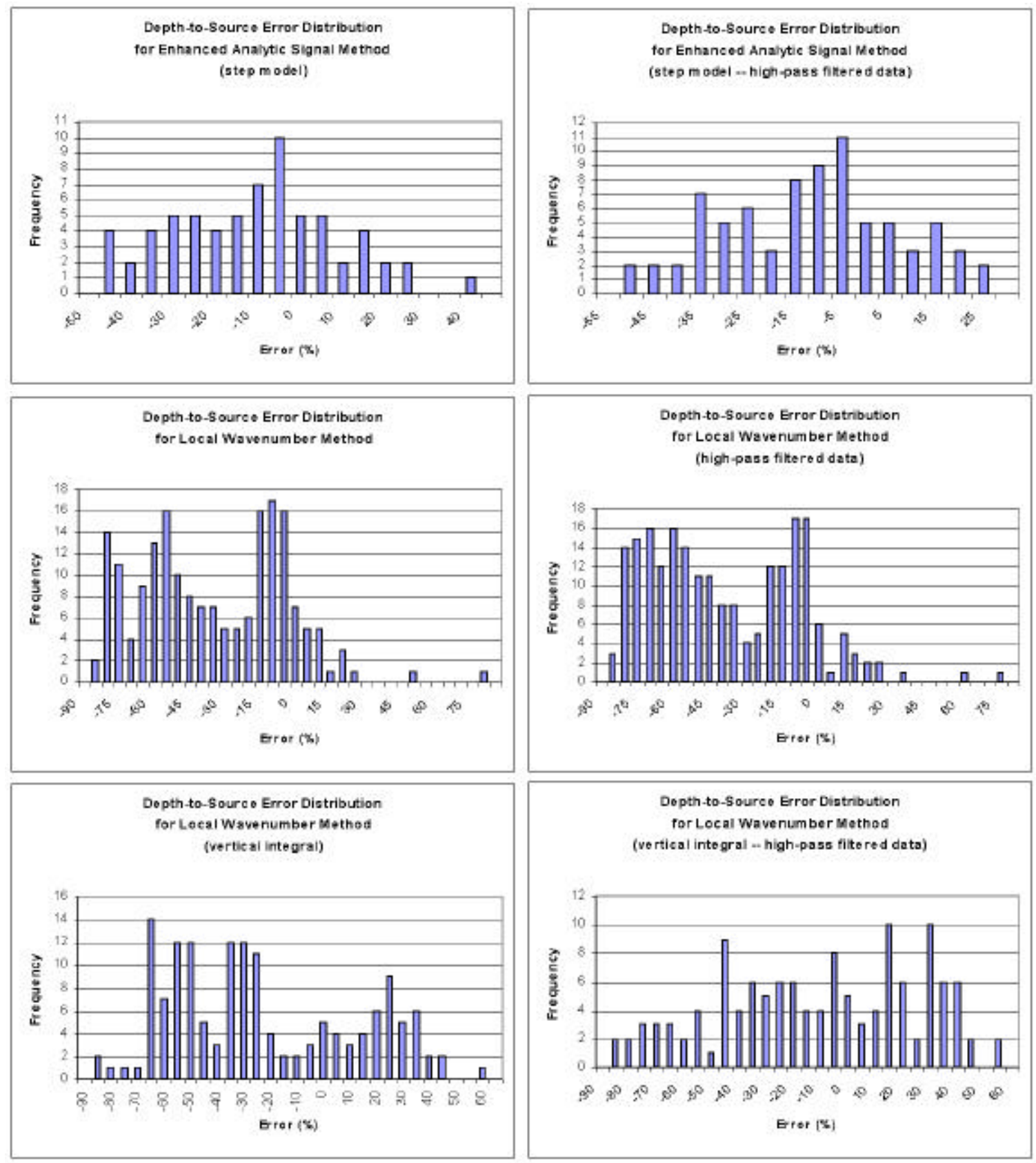
Figure 17, cont...
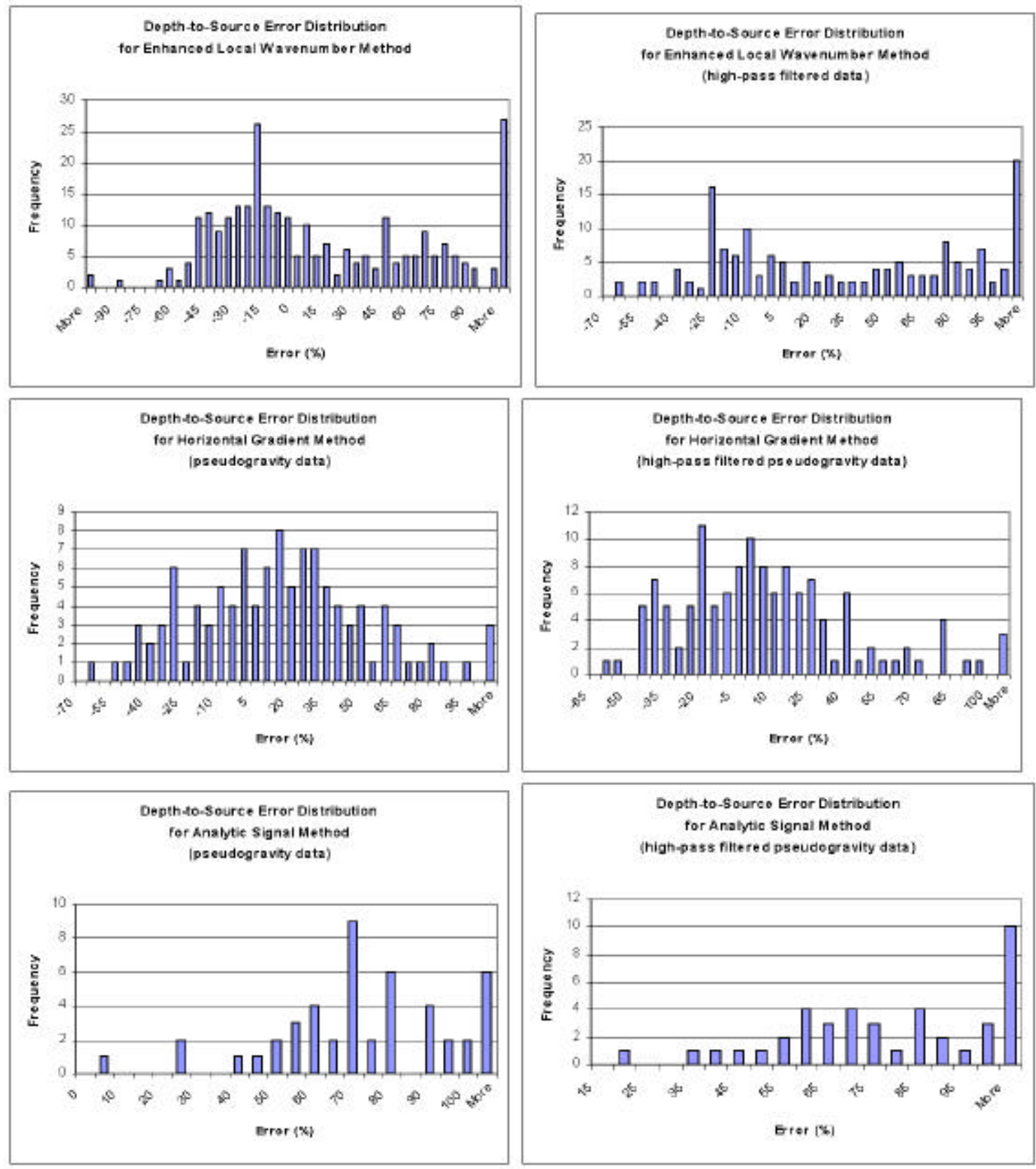
Figure 17, cont...
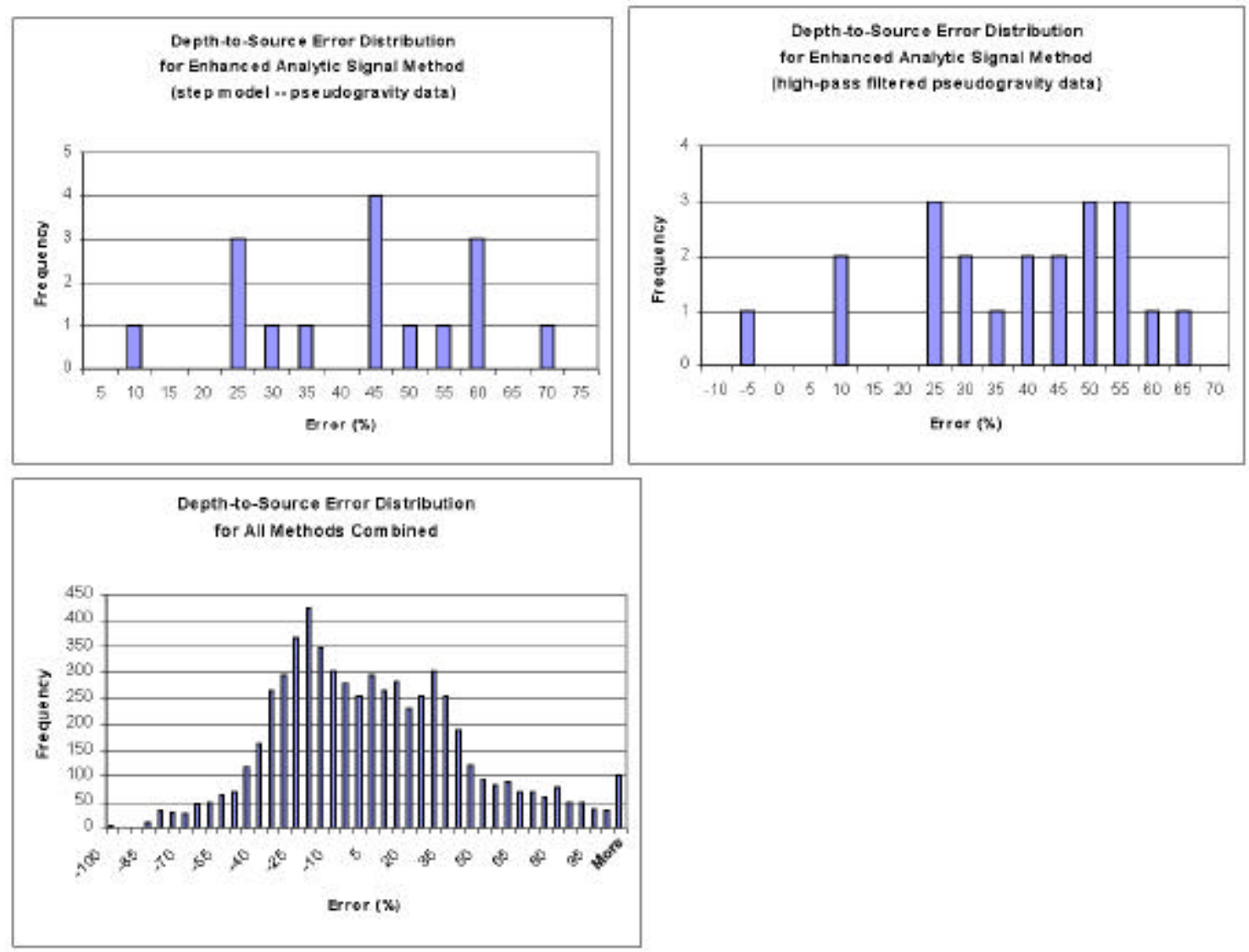


\section{Natural Gas Pipelines}

Two parallel pipelines run through southeastern Tucson and provide the city with natural gas (Figures 18 and 19). The pipelines are owned and maintained by the El Paso Natural Gas Company. In the study area, the two pipelines strike at approximately 30 degrees west of north, are separated by approximately six meters (20 ft) and are buried about one meter below the surface. One of the pipelines is about $3 / 4$ of a meter (30 in) in diameter, and the other has a diameter of about $2 / 3$ of a meter (26 in). Both pipelines possess cathodic protection, with a change in polarity occurring between the northwestern and southeastern sections of the study area.

The pipelines are relatively isolated from any other shallow magnetic sources, since they are located away from the center of town and associated cultural noise. The magnetic expression of the pipelines is one of a dipole with a declination that appears to be perpendicular to the strike of the pipelines. In the northwest, the dipole points to the northeast and has a declination of 30 degrees. In the southeast, the dipole points to the southwest and has a declination of 210 degrees. The magnetic anomaly has a total range in amplitude of nearly $15 \mathrm{nT}$, peak to trough (Figure 20). The major component of the observed field anomaly is considered to be the result of the current that runs through the pipelines for cathodic protection, with a very small induced-magnetization component. This makes the pipelines perfect targets for testing depth-to-source calculations on a shallow source with a purely induced field as the result of an applied current.

Since the two pipelines are relatively close to one another and appear as one pipeline at the observation elevation, the magnetic contact locations were considered to 


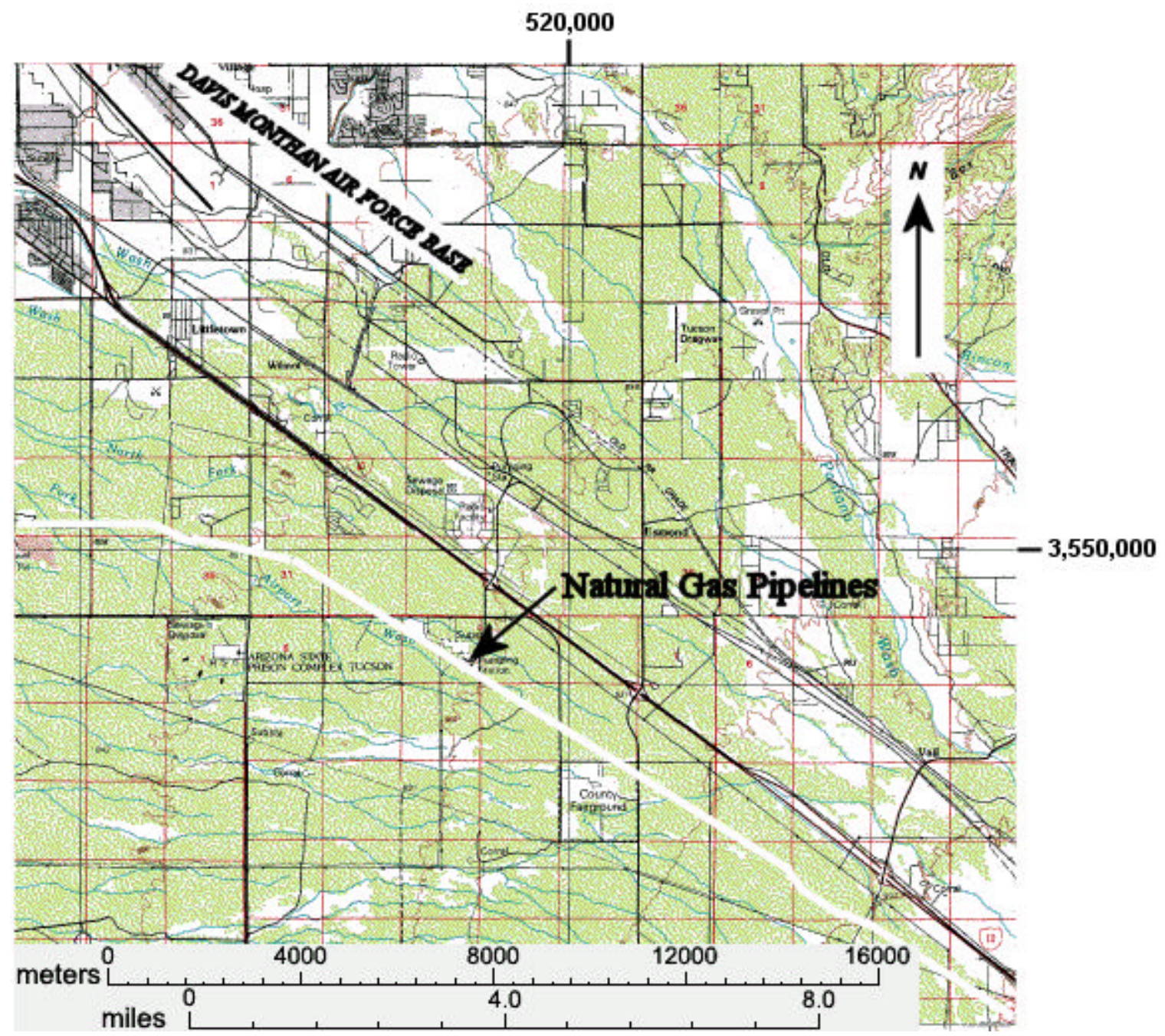

Figure 18. Location of the natural gas pipelines southeast of Tucson (modified from TopoZone, 2000). 


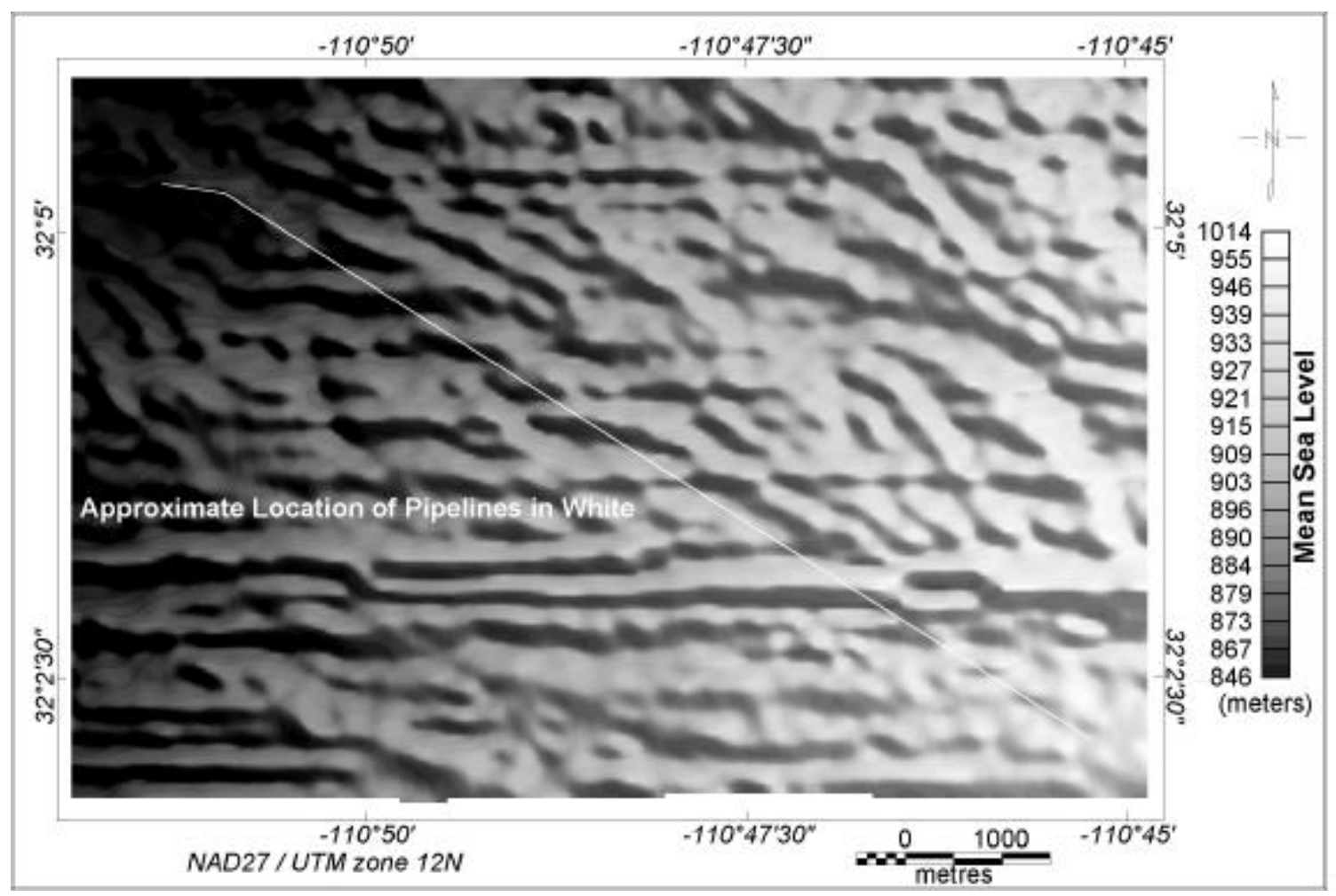

Figure 19. Topographic map created from radar altimeter and elevation data collected during the survey over the natural gas pipelines. 


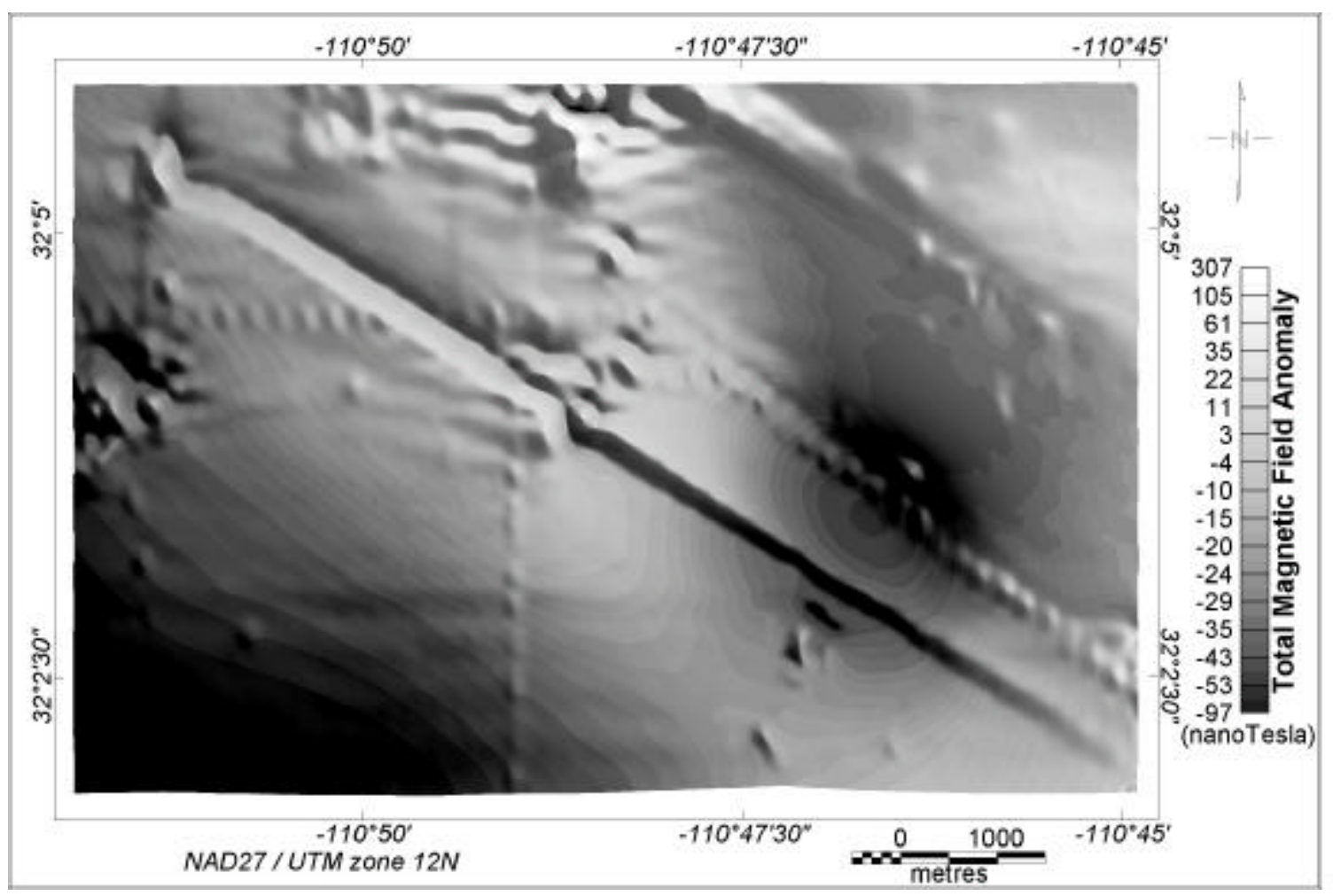

Figure 20. Total magnetic field anomaly map over the natural gas pipelines.

be at the outer edges of the two pipelines. These contacts are effectively at ground level. All of the methods discussed in this study were used to calculate the depth to the magnetic contact above the pipelines, and an error was calculated using the flight height as the correct solution.

The reduced magnetometer data were gridded with a 10-meter grid interval using the bi-directional line gridding algorithm in OASISmontaj. Before any derivatives were calculated, a 150-meter low-pass Butterworth filter, using an $8^{\text {th }}$ degree function, was applied to the gridded data. All signals were subsequently calculated from the lowpass filtered 10-meter grid. The grids were re-gridded at a larger interval before any depths were calculated. Before re-gridding, a low-pass linear convolution filter with a 
length of the new grid spacing was used in the direction of the grid lines. For all methods, a 20-meter grid interval was used to find magnetic contacts.

A structural index of 2 was used for Euler deconvolution, since the pipelines have only one infinite dimension. The search window size was set to 1000 meters with a maximum search radius of 500 meters, which windowed enough of the anomaly to calculate the depth without including interfering anomalies. For the enhanced analytic signal, the formula for an infinite step (Equation 32) as well as the formula for a dike (Equation 35) was used to calculate the depth. Table 3 summarizes the depth-to-source error results for each method. Appendix $\mathrm{C}$ contains the maps of the specific signals used for each calculation with the relevant calculated strikes and depths plotted as lines and circles, respectively. 
Table 3. Depth-to-source error statistics for the natural gas pipelines.

\begin{tabular}{|c|c|c|c|c|c|c|c|c|c|}
\hline Method & Median & Mean & Standard Dev. & Kurtosis & Skewness & Range & Minimum & Maximum & Count \\
\hline euler & 37.6 & 36.8 & 38.3 & 0.8 & 0.8 & 215.0 & -25.5 & 189.5 & 626 \\
\hline hpeuler & 80.7 & 84.5 & 16.8 & 0.6 & 1.0 & 88.4 & 56.0 & 144.4 & 1644 \\
\hline$h g$ & -30.8 & -27.4 & 16.5 & 2.4 & 1.4 & 102.8 & -63.1 & 39.7 & 781 \\
\hline hphg & -30.2 & -31.5 & 11.3 & 3.2 & 0.4 & 91.4 & -65.2 & 26.2 & 820 \\
\hline sas & -3.7 & 1.4 & 21.7 & 8.9 & 2.4 & 145.6 & -29.3 & 116.4 & 156 \\
\hline hpsas & -3.4 & -3.0 & 11.1 & -0.4 & 0.1 & 50.1 & -27.9 & 22.2 & 118 \\
\hline step eas & -68.7 & -67.5 & 21.5 & -0.5 & 0.5 & 96.4 & -97.8 & -1.4 & 203 \\
\hline step hpeas & -27.1 & -34.9 & 20.8 & -0.1 & -0.8 & 89.3 & -85.6 & 3.7 & 147 \\
\hline dike eas & -114.2 & -123.4 & 104.0 & 4.3 & -0.4 & 813.0 & -607.8 & 205.2 & 202 \\
\hline dike hpeas & -24.6 & 263.5 & 5450.8 & 115.7 & 10.4 & 71572.1 & -11389.6 & 60182.6 & 130 \\
\hline Iwn & -27.6 & -27.4 & 17.3 & 0.7 & 0.1 & 113.3 & -79.3 & 34.1 & 227 \\
\hline hplwn & -23.2 & -22.8 & 17.4 & 0.8 & 0.2 & 103.0 & -73.0 & 30.0 & 267 \\
\hline vilwn & -54.8 & -47.1 & 25.5 & -0.2 & 0.9 & 108.5 & -79.7 & 28.8 & 377 \\
\hline hpvilwn & 14.1 & 10.8 & 26.1 & 0.2 & -0.1 & 139.4 & -47.4 & 92.0 & 226 \\
\hline elwn & 82.9 & 109.6 & 118.4 & 6.1 & 1.9 & 810.5 & -69.6 & 740.9 & 302 \\
\hline hpelwn & 65.4 & 64.4 & 29.5 & 1.3 & 0.1 & 200.9 & -31.8 & 169.1 & 333 \\
\hline hgpg & 54.9 & 92.6 & 85.5 & 0.4 & 1.2 & 377.1 & -18.6 & 358.5 & 246 \\
\hline hphgpg & -4.6 & -4.9 & 6.6 & 3.2 & -1.0 & 50.6 & -36.8 & 13.9 & 388 \\
\hline saspg & 45.2 & 64.2 & 45.4 & 0.2 & 1.2 & 203.5 & 3.3 & 206.8 & 160 \\
\hline hpsaspg & 36.8 & 35.3 & 16.8 & 1.1 & -0.7 & 87.2 & -15.6 & 71.6 & 133 \\
\hline step easpg & -79.8 & -76.8 & 15.4 & 9.1 & 2.3 & 89.4 & -96.1 & -6.7 & 43 \\
\hline step hpeaspg & -57.5 & -56.0 & 17.6 & 2.8 & 0.9 & 118.3 & -90.2 & 28.0 & 153 \\
\hline dike easpg & -111.6 & -112.9 & 7.4 & -0.9 & -0.4 & 26.4 & -128.4 & -102.0 & 42 \\
\hline dike hpeaspg & -139.4 & -152.2 & 37.7 & 1.8 & -1.4 & 180.7 & -285.8 & -105.1 & 107 \\
\hline \multicolumn{4}{|c|}{ euler - euler deconvolution } & \multicolumn{6}{|c|}{ hp - calculated from high-pass filtered data } \\
\hline \multirow{2}{*}{\multicolumn{4}{|c|}{$\begin{array}{ll}\text { hg } & \text { - horizontal gradient } \\
\text { sas } & \text { - simple analytic sianal }\end{array}$}} & \multirow{2}{*}{\multicolumn{6}{|c|}{ pg - calculated from pseudo-gravity data }} \\
\hline & & & & & & & & & \\
\hline \multicolumn{4}{|c|}{ eas - enhanced analytic signal } & \multicolumn{6}{|c|}{ step - calculated using the infinite step model } \\
\hline \multicolumn{4}{|c|}{$\begin{array}{l}\text { Iwn - local wavenumber } \\
\text { elwn - enhanced local wavenumber }\end{array}$} & \multicolumn{6}{|c|}{ dike - calculated using the dike model } \\
\hline
\end{tabular}

Figure 21 shows the depth-to-source error histograms for all of the methods used. The histograms for Euler deconvolution reveal that most of the depth estimates (78\%) are too deep. Applying a high-pass filter to the magnetic anomaly data before calculating depth estimates eliminated many of the shallower depth estimates and introduced many deeper estimates, resulting in all of the depth estimates occurring too deep. Also, the number of depth estimates more than doubled after applying a high-pass filter. Most of the depth estimates from the horizontal gradient (93\%) and local 
wavenumber (94\%) methods are too shallow. Filtering seems to have had little effect on the number and distribution of depth estimates. For the simple analytic signal method, most of the depth estimates $(61 \%)$ are too shallow, but close to the true depth to source. A high-pass filter removed both shallow solutions and many of the very deep solutions, which still resulted in most of the solutions $(62 \%)$ occurring too shallow. The step-model enhanced analytic signal method resulted in all of the depth estimates occurring too shallow. Filtering removed many of the shallower solutions and introduced some deeper depth estimates, but nearly all of them (99\%) remain too shallow. The dike-model enhanced analytic signal method resulted in very sporadic error distributions, with many errors greater than $100 \%$ and less than $-100 \%$. Filtering seems to have introduced even greater errors. Most of the depth estimates (94\%) from the local wavenumber method applied to the vertical integral of the total-field magnetic anomaly data are too shallow. Applying a high-pass filter before calculating depth estimates resulted in the removal of shallower depth estimates and introduced many deeper estimates, resulting in a distribution that is fairly even about a common value close to the true depth to source and most of the depth estimates $(71 \%)$ occurring too deep. For the enhanced local wavenumber method, most of the depth estimates (87\%) are too deep and the error distributions are very sporadic with many errors greater than 100\%. Applying a highpass filter resulted in the removal of a few shallow solutions and introduced a few deep solutions and most of the depth estimates (98\%) occurring too deep. The horizontal gradient method applied to the pseudo-gravity anomaly field resulted in most of the depth estimates (95\%) occurring too deep. Applying a high-pass filter to the data removed 
many of the deeper solutions and introduced more shallow solutions, resulting in depth estimates clustering around a value closer to the true depth to source and most of them (80\%) occurring too shallow. The simple analytic signal method applied to the pseudogravity anomaly field yielded solutions that were all too deep. A high-pass filter removed many of the deeper solutions and introduced a few shallower solutions, but still resulted in most of the depth estimates (95\%) occurring too deep. Both the step-model and dike-model enhanced analytic signal methods applied to the total-field pseudogravity anomaly data yielded solutions that are much too shallow, with no depth estimates occurring on the positive side of the error distributions. A high-pass filter introduced some deeper depth estimates, which are still too shallow, for the step model, while many shallower estimates were introduced for the dike model. 
Figure 21. Depth-to-source error histograms for each method over the natural gas pipelines.
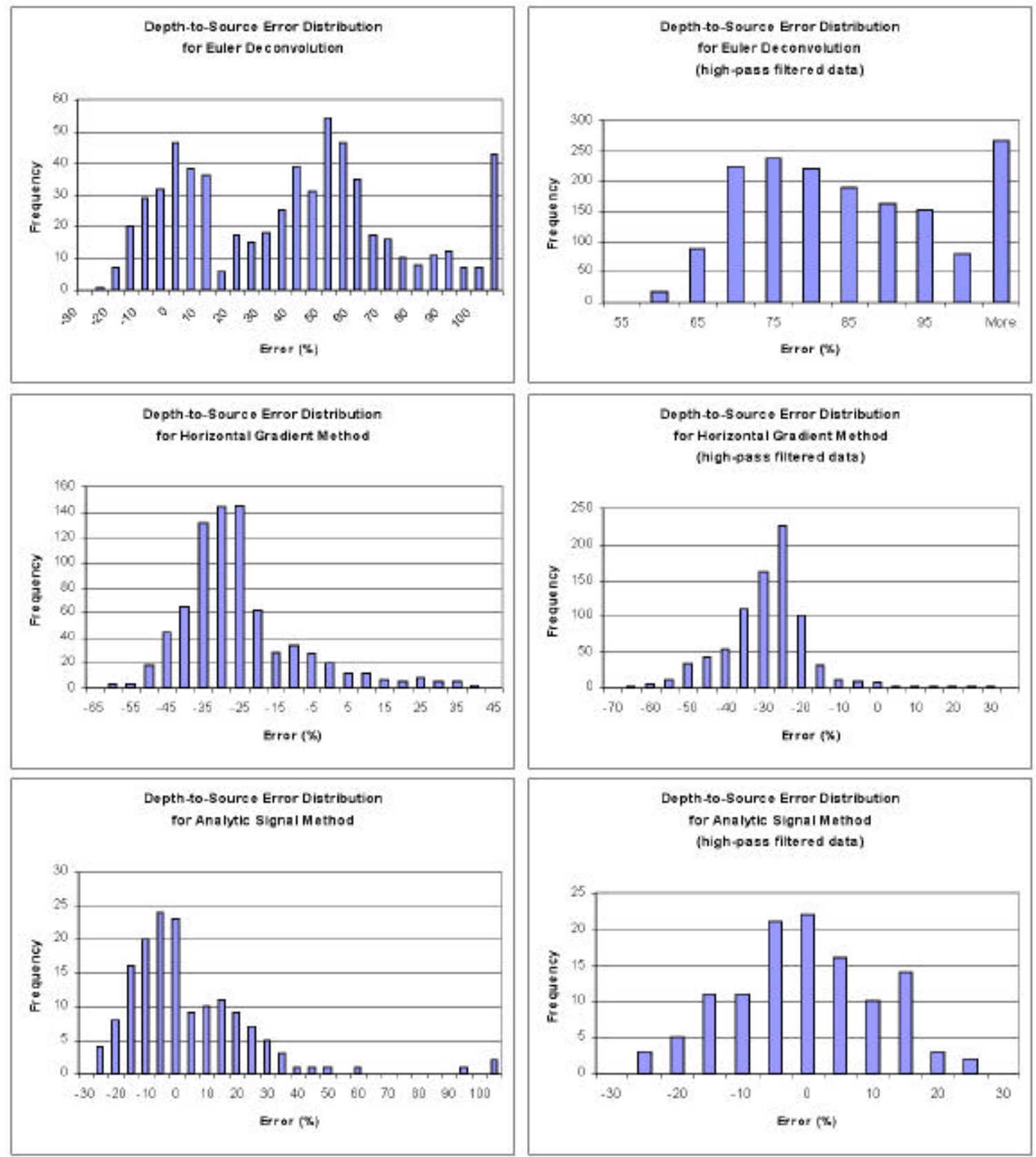
Figure 21, cont...
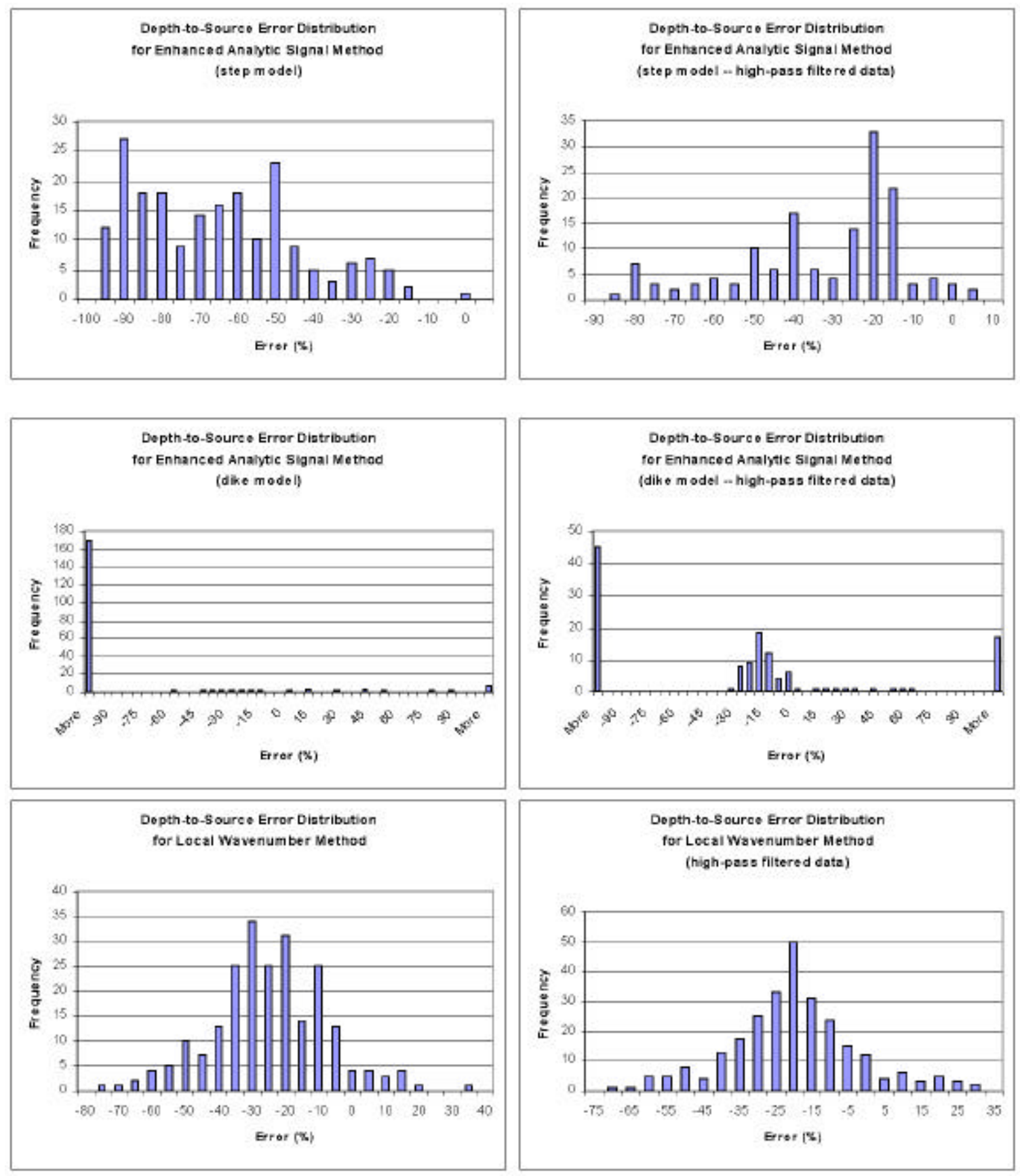
Figure 21, cont...
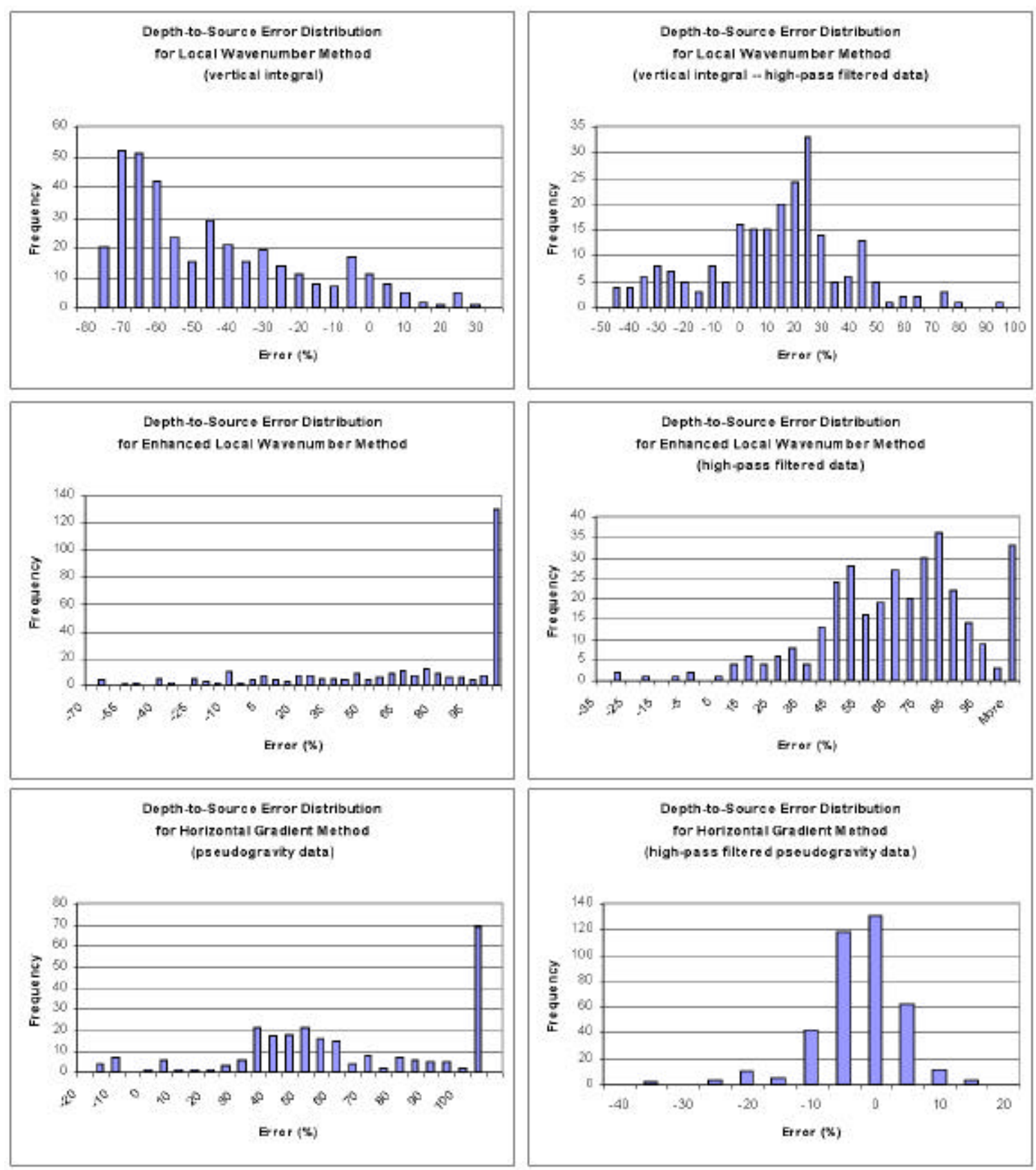
Figure 21, cont...
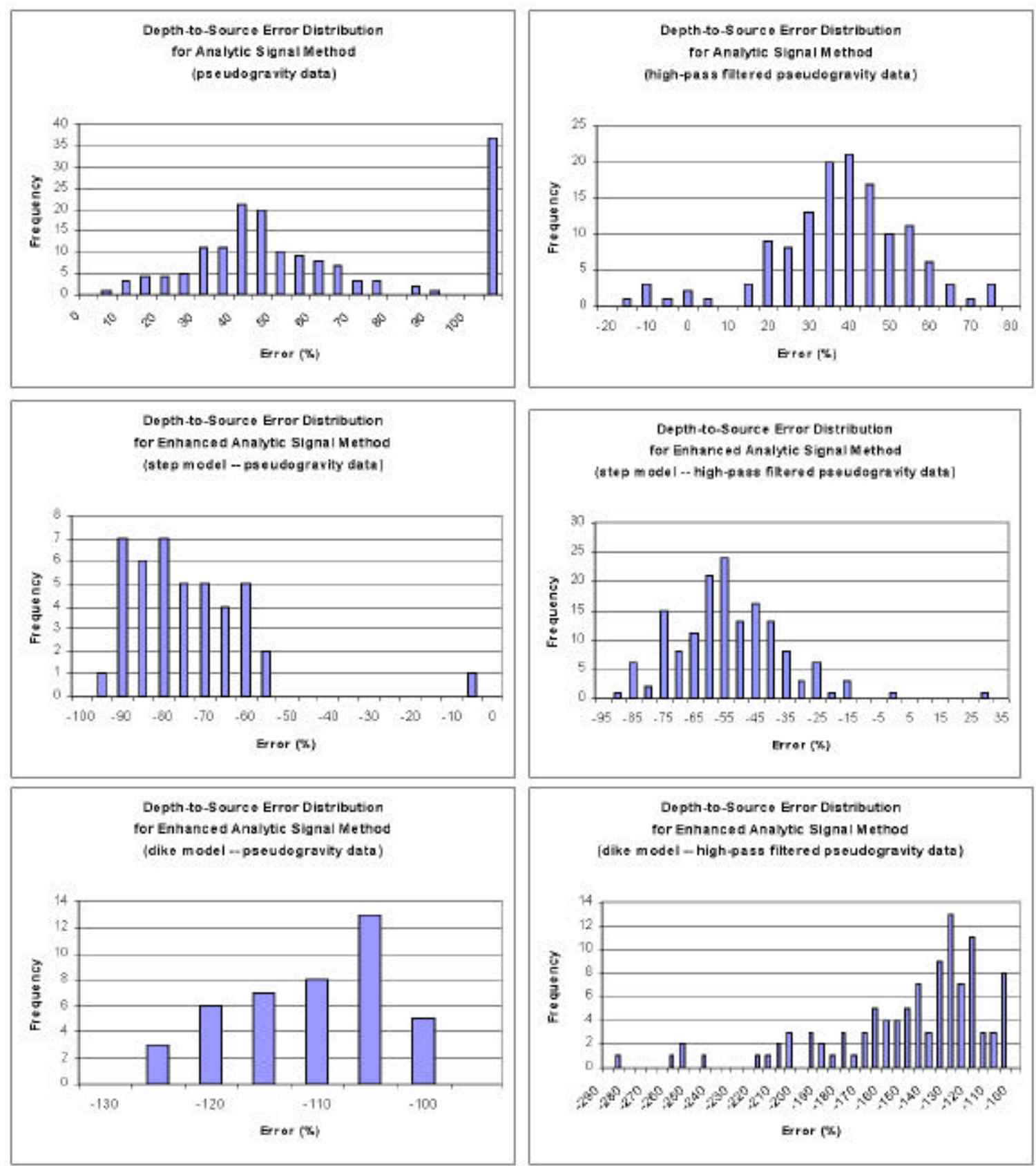


\section{DISCUSSION}

\section{Gridding and Filtering}

Through much trial and error, it was determined that careful attention to gridding and filtering was necessary to achieve good results. This fact became even clearer as high-order derivatives of the gridded data were calculated.

The top image in Figure 22 is the simple analytic signal amplitude over the landfill that was calculated with a 5-meter grid of the reduced magnetometer data. Line bias, due to the higher sampling in the along line directions as opposed to the across line direction, has introduced a lot of noise that has expressed itself as vertical striping in the image. The bottom image in Figure 22 is the second order analytic signal amplitude calculated from the same data. The signal is masked by high amplitude, high-frequency noise in both vertical and horizontal directions within the image. It was necessary to remove any high-frequency noise that was present in the original data in order to reduce this computational noise. 

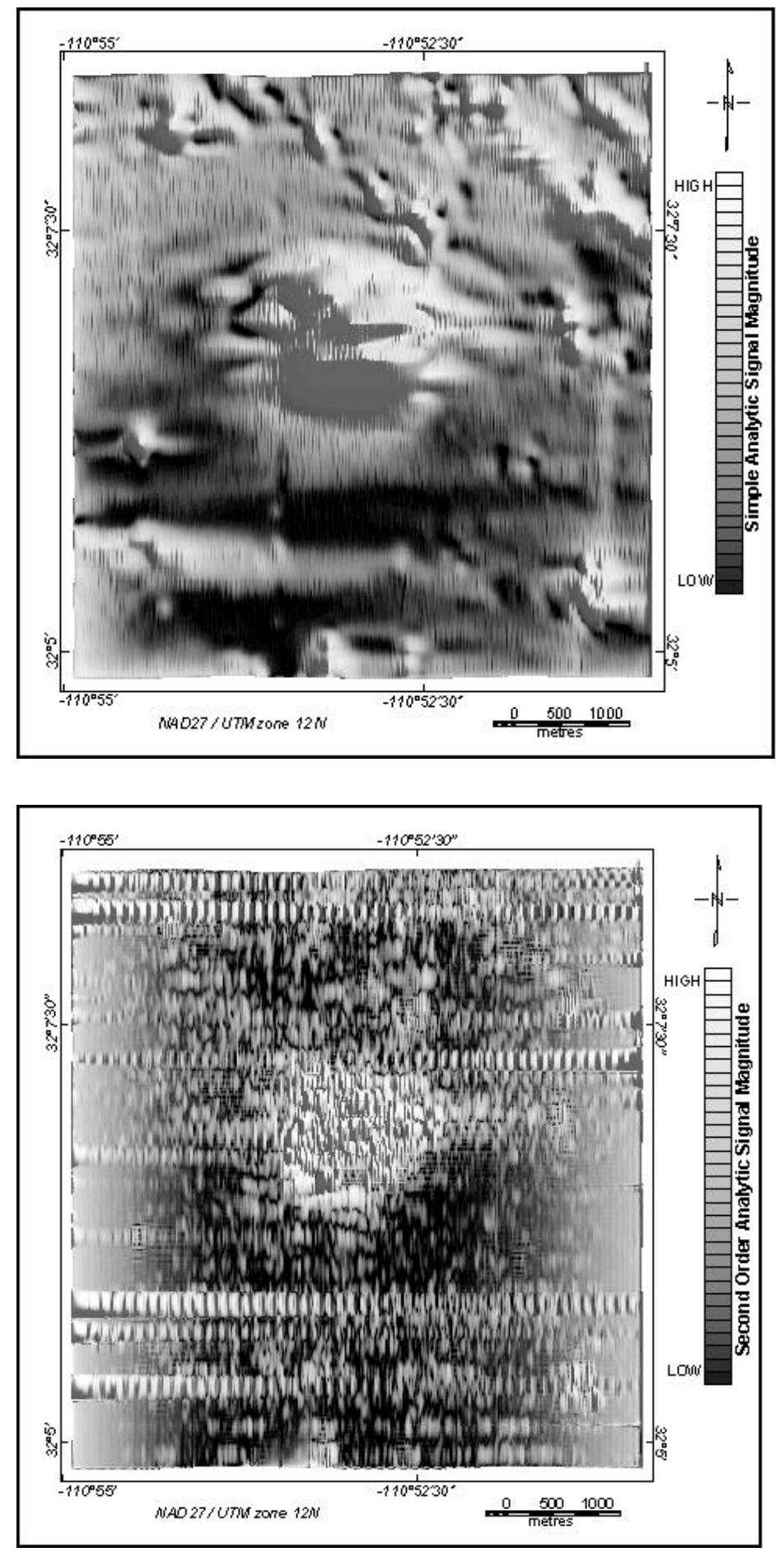

Figure 22. The simple (top) and second-order (bottom) analytic signal amplitudes over the Los Reales Landfill calculated from a 5-meter grid. Notice the vertical stripes that are due to the down-line sampling bias. 
To reduce the introduction of computational noise, it was necessary to apply a low-pass filter to the data before any derivatives were calculated. Applying a low-pass filter only in the down-line direction eliminated the vertical striping in the simple analytic signal image by compensating for the down-line sampling bias. However, the secondorder analytic signal continued to suffer from high-amplitude noise. Figure 23 shows the simple and second order analytic signal amplitudes over the landfill that were calculated after a Butterworth low-pass filter had been applied to the gridded magnetometer data. Most of the high amplitude noise has been eliminated from the signal. A cutoff wavelength of 150 meters (same as the line spacing) and an $8^{\text {th }}$ degree function were used as the filter parameters. These parameters were chosen based on the Nyquist frequency of the line spacing. Since the line spacing for the aeromagnetic data is 150 meters, any anomalies that have a wavelength smaller than 300 meters in the across-line direction are not properly represented in the gridded data. It was reasonable, then, to eliminate all wavelengths smaller than 300 meters from the magnetometer data. The anomalies of interest were much larger than this, so as not to be affected by the removal of such wavelengths. The filter parameters used preserved more than $98 \%$ of the amplitude of anomalies having a wavelength of 300 meters and provided a smooth enough function to prevent the occurrence of Gibb's phenomena (ringing in the data). This made the line spacing a good choice for the cutoff wavelength when removing high frequency noise from the data using a Butterworth filter. 

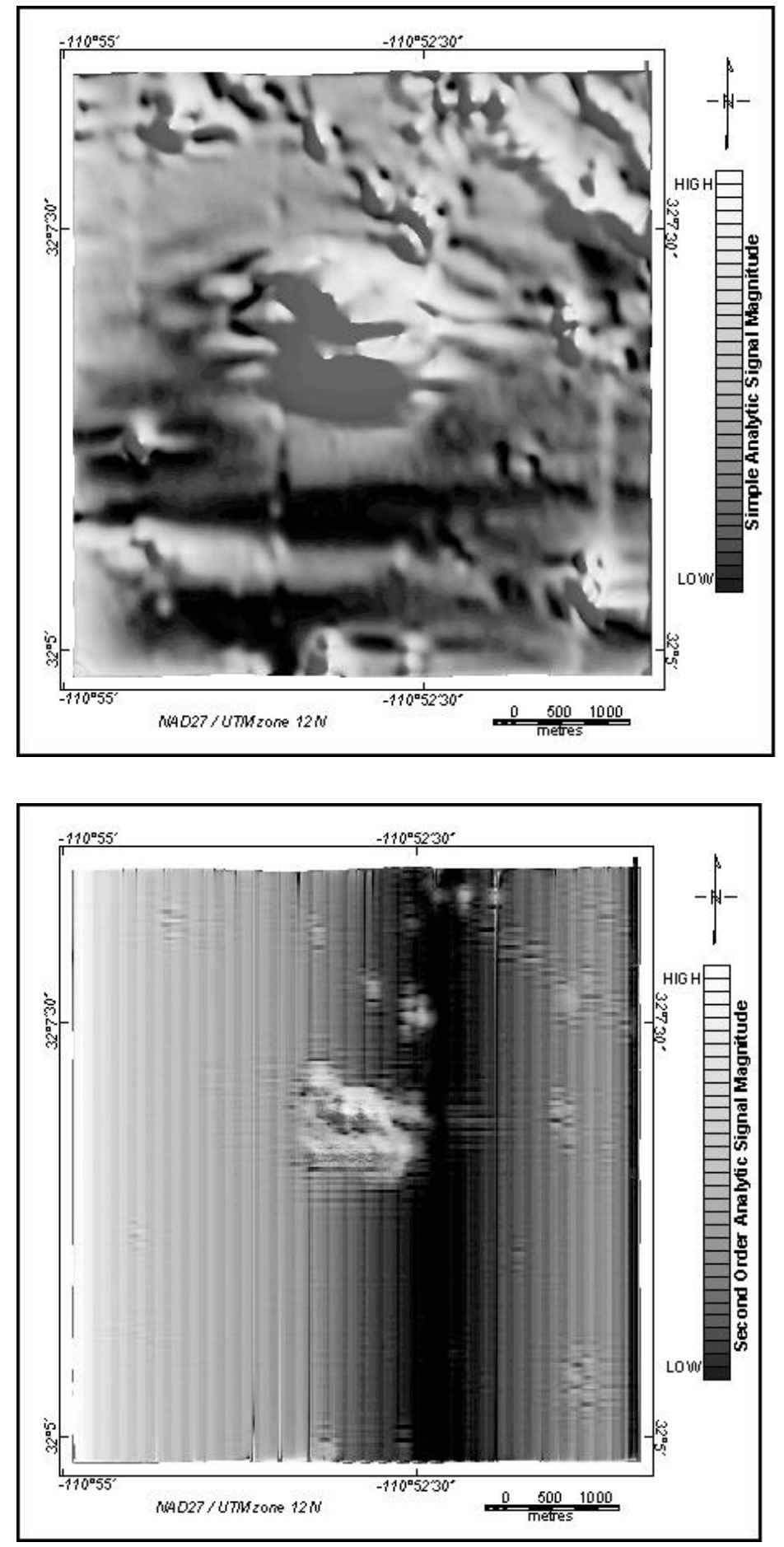

Figure 23. The simple (top) and second-order (bottom) analytic signal amplitudes over the Los Reales Landfill calculated from a low-pass filtered 5-meter grid. 
It should be noted that applying a low-pass filter to the data before calculating derivatives will affect the resulting depth estimates. The effects of such filtering can be seen in many of the depth-to-source error histograms (Figures 13, 17 and 21). Many of the histograms show a "tail" towards deeper solutions that is not seen towards the shallower solutions. The filter described previously should not, in theory, greatly affect the signals of interest due to the size of the anomalies relative to the filter parameters. However, the removal of smaller sources affects the resulting error distributions and may bias interpretation by implying that the source of interest is deeper than the true depth.

It is often suggested in the literature that the data be upward continued to reduce this noise (Reid et al., 1990; Hsu et al., 1998; Phillips, 2001). However, for shallow sources such as those in this study, an upward continuation would not be appropriate. An upward continuation in these cases would lead to a loss of valuable resolution and signal strength. Figure 24 shows the total magnetic field anomaly due to the landfill at the flight elevation and at an upward continuation of 150 meters (same as the line spacing). Although much of the noisy data has been removed, the total field at the upward continued level is less defined than that at the flight elevation and leads to fewer depth solutions (Figure 25). 

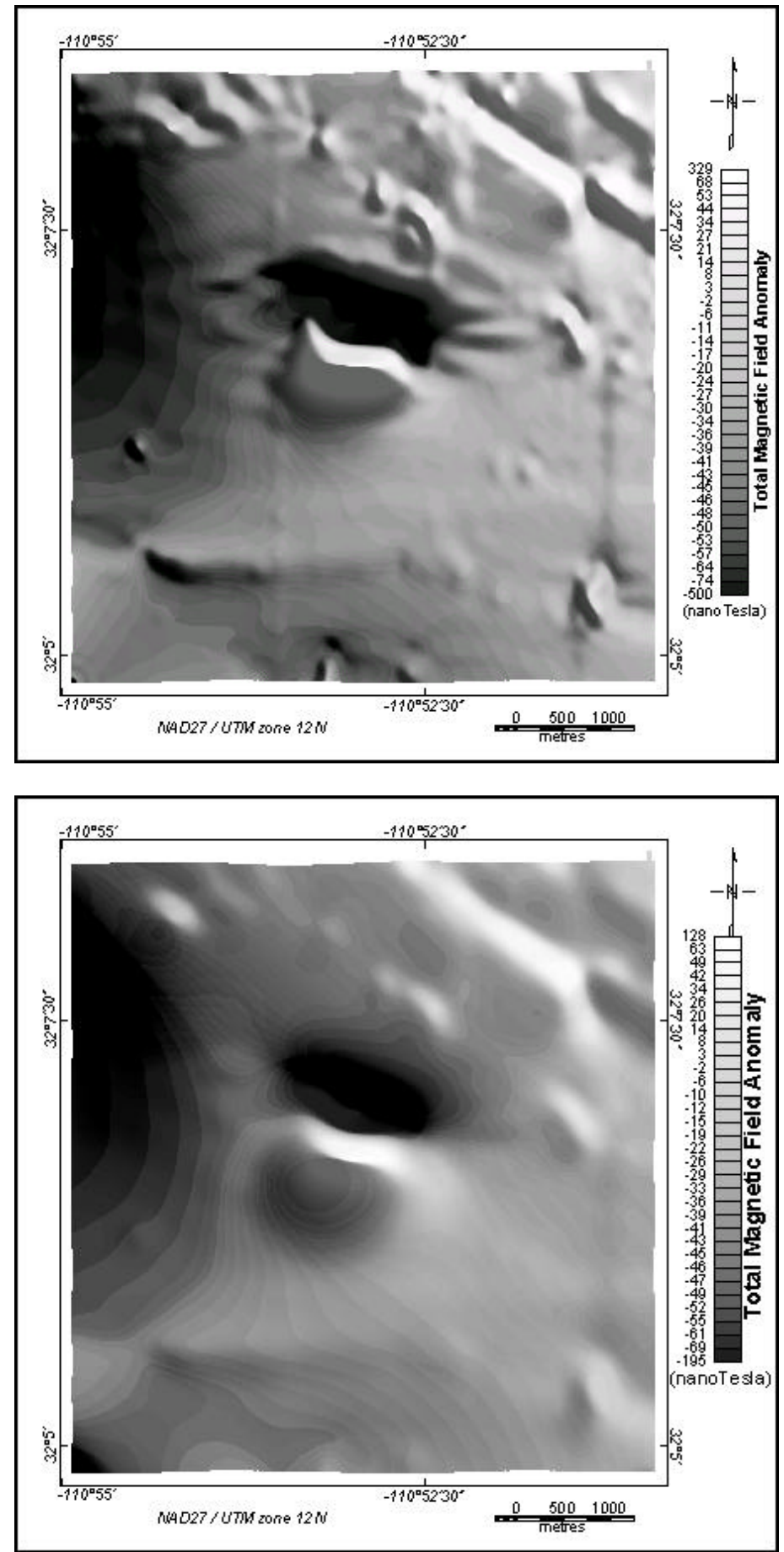

Figure 24. The total magnetic field anomaly due to the landfill at the flight elevation (top) and at an upward continuation of 150 meters (bottom). 

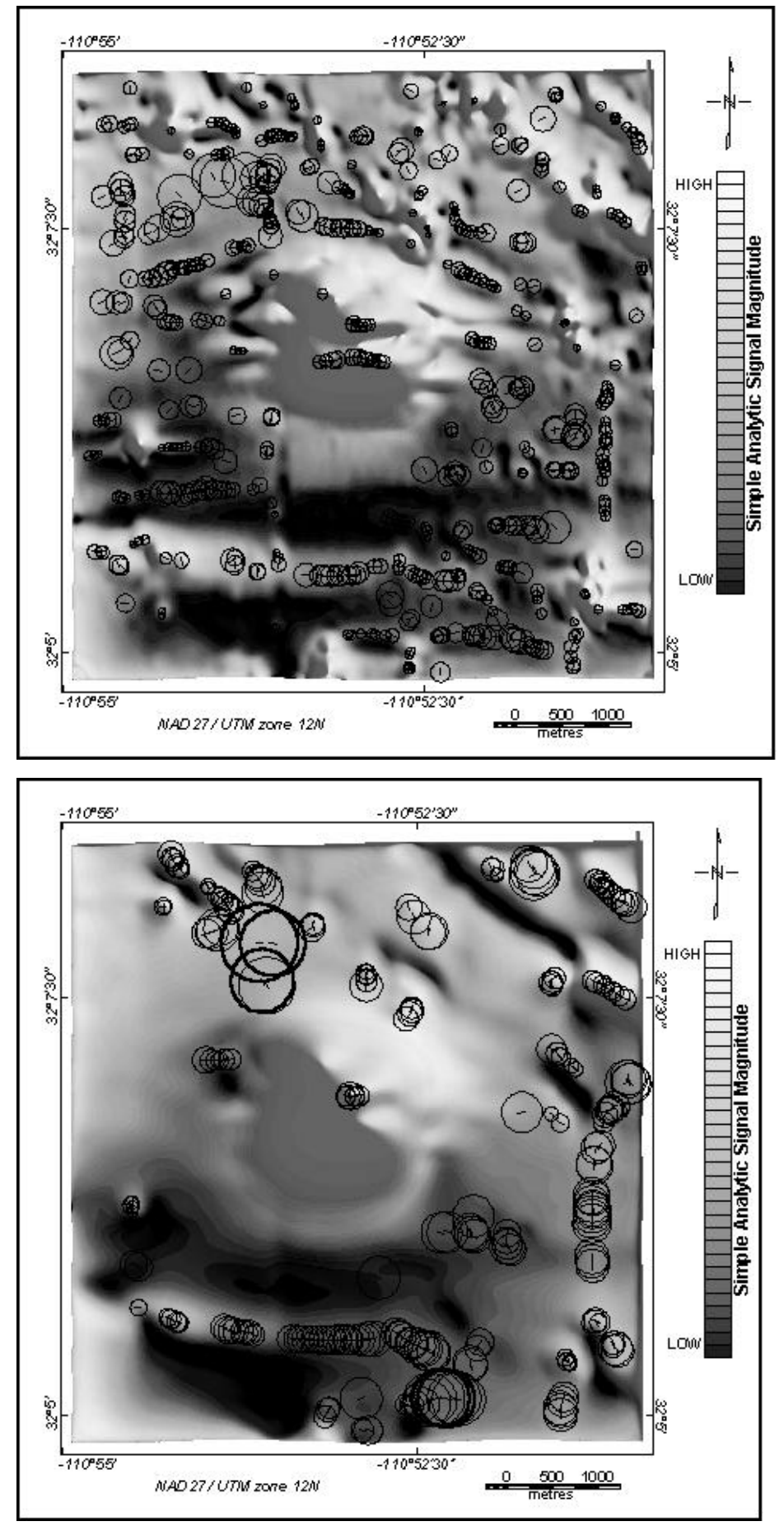

Figure 25. Comparison of the number of depth estimates using the simple analytic signal method over the landfill at the flight elevation (top) and at an upward continuation of 150 meters. Both images were gridded at 50 meters and only depth estimates with errors less than $15 \%$ were retained. 
The choice of a proper grid spacing when calculating derivatives was also important in achieving good results. It has been suggested that a distance of $1 / 4^{\text {th }}$ to $1 / 3^{\text {rd }}$ of the line spacing is the proper choice for the grid spacing when dealing with line data (Billings and Richards, 2000). Since the line spacing of the aeromagnetic data is 150 meters, a good choice for the grid spacing would be 50 meters. Calculating derivatives at this grid spacing left much to be desired in terms of precision, however. The top image in Figure 26 shows the second order analytic signal of the landfill that was calculated with a 50-meter grid spacing. The bottom image was calculated with a 5-meter grid spacing, then re-gridded to 50 meters. The noise and lack of precision for the 50 -meter analytic signal was due to the finite-difference algorithm that calculated the spatial derivates. A larger grid spacing encompassed too much of the curve and yielded poor results. This becomes most evident when calculating higher-order derivatives. It was best to grid the data at an interval that was small enough to incorporate most of the anomaly curvature. Intervals less than one percent of the anomaly wavelength worked well.

After the derivatives and signals of interest had been calculated at a small grid interval, it was necessary to re-grid the data at a larger interval for the purpose of locating the magnetic contacts. The choice of grid spacing was completely dependant upon the size of the search window used to find peaks in the signal of interest. Most of the algorithms used in this study incorporated a $5 \times 5$ data search window, so it was necessary to choose a grid size that allowed the search window to cover enough of the signal peaks without covering adjacent peaks. Choosing a grid size that was too small resulted in too 
many solutions, many of which were over very localized peaks due to noise in the image. Choosing a grid size that was too large resulted in too few solutions because of interference between adjacent peaks (Figure 27). 

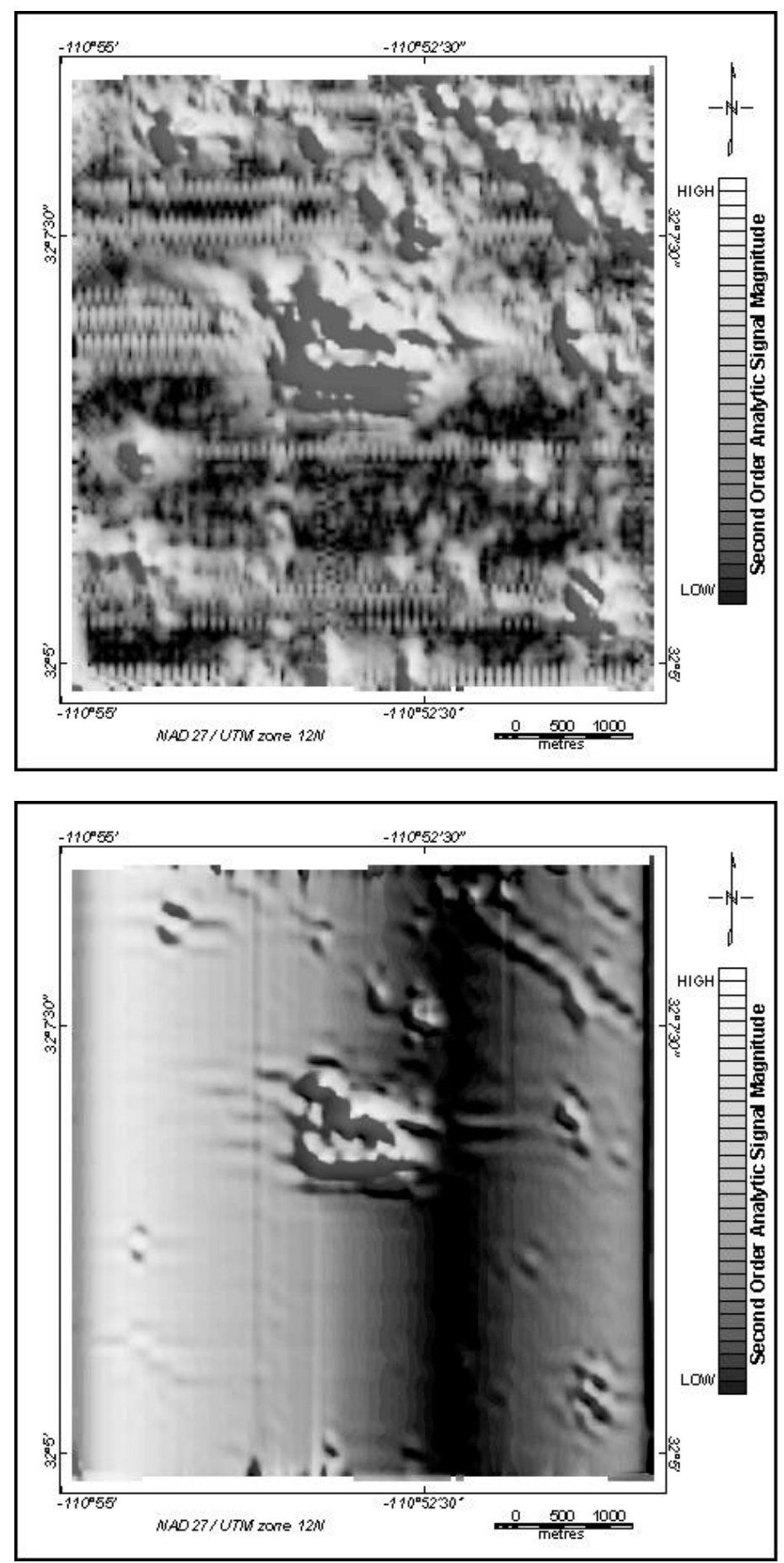

Figure 26. The second-order analytic signal amplitude over the landfill that was calculated with a 50-meter grid spacing (top) and calculated with a 5-meter grid spacing, then re-gridded at 50 meters (bottom). 

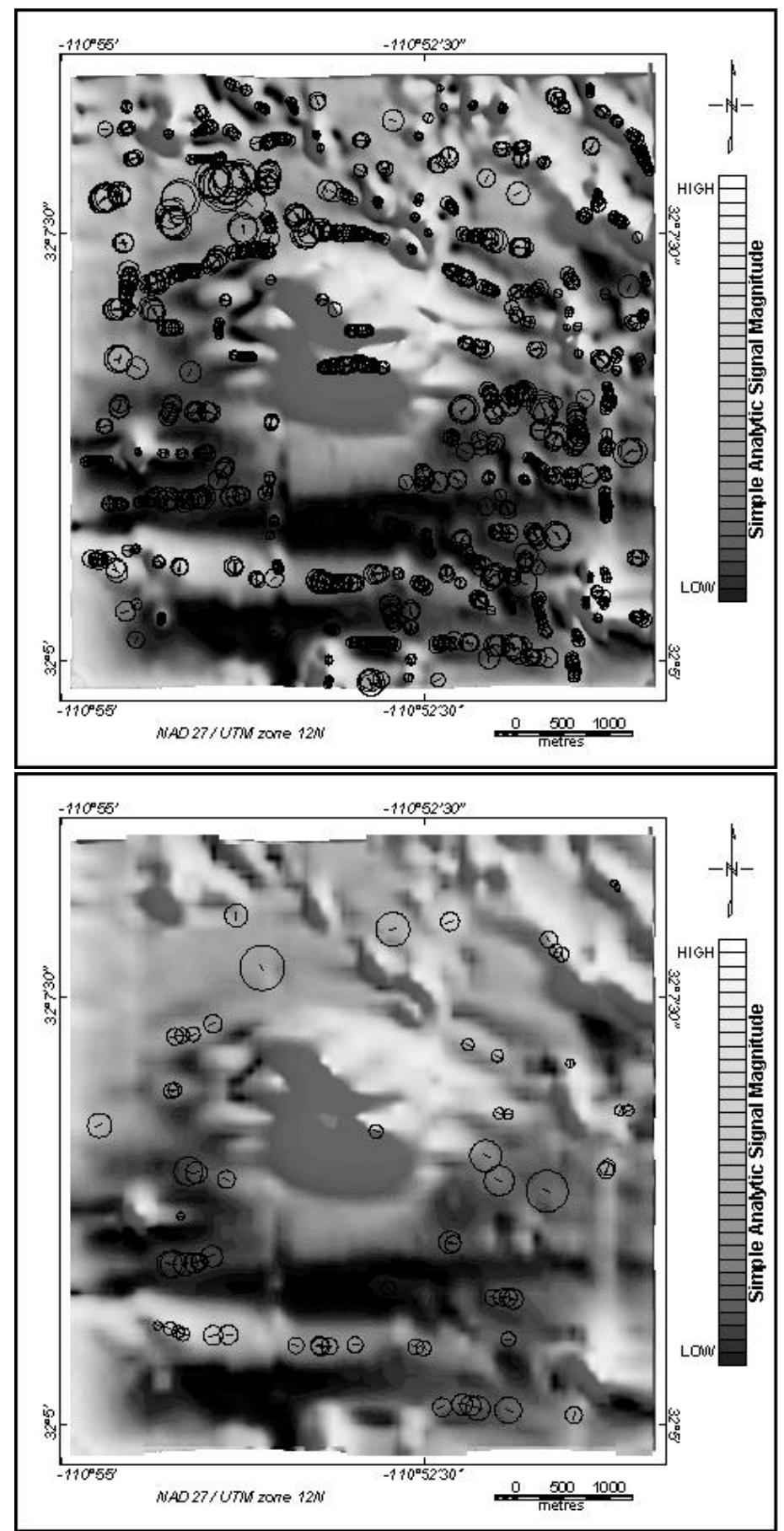

Figure 27. Comparison of the number of depth estimates using the simple analytic signal method over the landfill with different search-window sizes. The top image was gridded at 25 meters (i.e. 125 meter search window) and the bottom image was gridded at 100 meters (i.e. 500 meter search window). Only depth estimates with errors less than $15 \%$ were retained. 
Calculating derivatives introduced noise in the grid line directions. The frequency and amplitude of this noise was proportional to the grid spacing itself, with a smaller grid spacing resulting in high frequency, lower amplitude noise. It was necessary to smooth out this noise before re-gridding for the purpose of locating magnetic contacts. This was done by applying a linear low-pass convolution filter in the grid line directions before regridding. The convolution filter used in OASISmontaj was designed using the method of Fraser et al. (1966). The default length was as long as the specified cutoff wavelength, which produced a filter that exhibited a minimum of side effects, such as Gibb's phenomena. The dashed line in Figure 28 is part of a profile of the second order analytic signal over the landfill that was calculated with a 5-meter grid. The image was regridded at the same grid spacing with a linear low-pass filter of length 20 meters applied in the grid line directions before re-gridding. The solid line in Figure 28 is the same profile of the filtered image over the landfill. All of the high frequency noise has been removed from the data, and the primary signal has been preserved.

\section{Second Order Analytic Signal Profile}

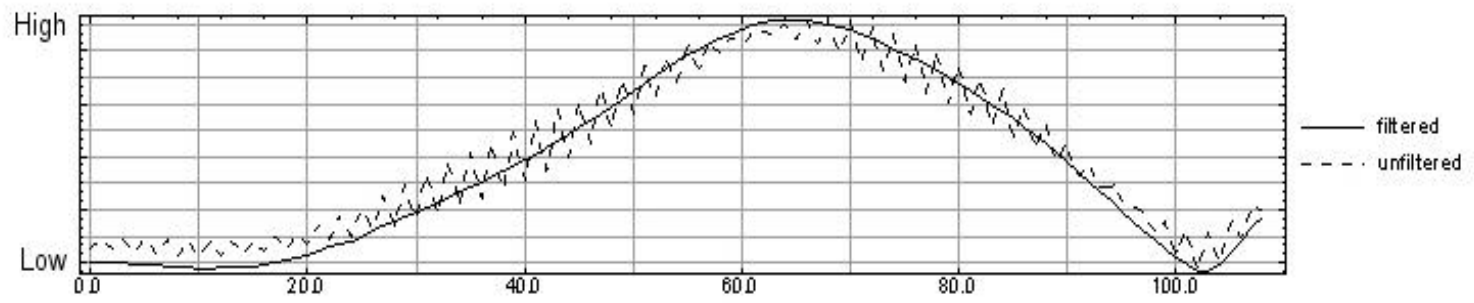

Figure 28. Profile of the second order analytic signal amplitude over the Los Reales Landfill before and after applying a low-pass filter. 


\section{Best and Worst Methods}

To determine which methods worked the best for estimating the correct depth to source, the distributions of the depth-to-source errors were examined. The main assumption was that given a large enough sample of a population of depth estimates, the error values would be clustered around a central value, and the frequency of occurrence would decline away from this central point. In many of the test cases, a bell-shaped distribution, one approaching a normal distribution, was evident where there were a significant number of depth-to-source errors obtained (Figures 13, 17, and 21).

Tables 1 through 3 provide values for skewness and kurtosis of the depth-tosource error distributions for each method. These values describe the shape and symmetry of a distribution and can be used to compare any distribution with a normal distribution. Skewness is defined by the third statistical moment about the mean and describes the asymmetry of a distribution. A normal distribution has a skewness value of zero. A positively skewed distribution has a majority of values greater than the mean value, while a negatively skewed distribution has a majority of values less than the mean value. Kurtosis is defined by the fourth statistical moment about the mean and describes the peakedness or flatness (i.e. weight of the tails) of a distribution. In Tables 1 through 3, a normal distribution has a kurtosis value of zero. A positive kurtosis value describes a distribution that is peaked, while a negative value describes a distribution that is flat. Since there are no clear definitions of distributions for specific values of skewness and kurtosis (other than values of zero describing a normal distribution), these values should only be used to compare multiple distributions and to determine their relative proximity 
to a normal distribution. Also, it should be noted that these values only carry significance if there is a substantially large sample size (e.g. >30).

Each depth-to-source method was rated independently over each test site. The rating was based on the median depth-to-source error for the particular method over the particular test site (Figure 29). The median was chosen over the mean error as the determining value to compensate for any outliers that may exist far from the central distribution of depth errors. In many cases, there were several outliers that clearly did not belong to the distribution of interest. These error values were most likely due to noise in the original data, computational noise or depths from deeper sources. The ratings of the depth-to-source methods over each test site ranged from excellent to very poor (Figure 30) and were based on the following criteria:

$$
\begin{aligned}
\text { median error } \leq 5 \% & \rightarrow \text { excellent } \\
5 \%<\text { median error } \leq 10 \% & \rightarrow \text { good } \\
10 \%< & <\text { median error } \leq 15 \% \rightarrow \text { fair } \\
15 \%< & \text { median error } \leq 50 \% \rightarrow \text { poor } \\
\text { median error }>50 \% & \rightarrow \text { very poor. }
\end{aligned}
$$

The methods that yielded the best results, those rated fair or better, for all three test sites included the simple analytic signal method applied to the total and high-pass filtered magnetic anomaly field, the local wavenumber method applied to the vertical integral of the high-pass filtered magnetic anomaly field and the horizontal gradient method applied to the high-pass filtered pseudo-gravity anomaly field. The natural gas pipelines may be considered a very special case, however, since their magnetic field is the direct result of an applied current. This type of magnetic field does not behave in the same way as a magnetic field induced by a primary magnetic field. If only the Los 


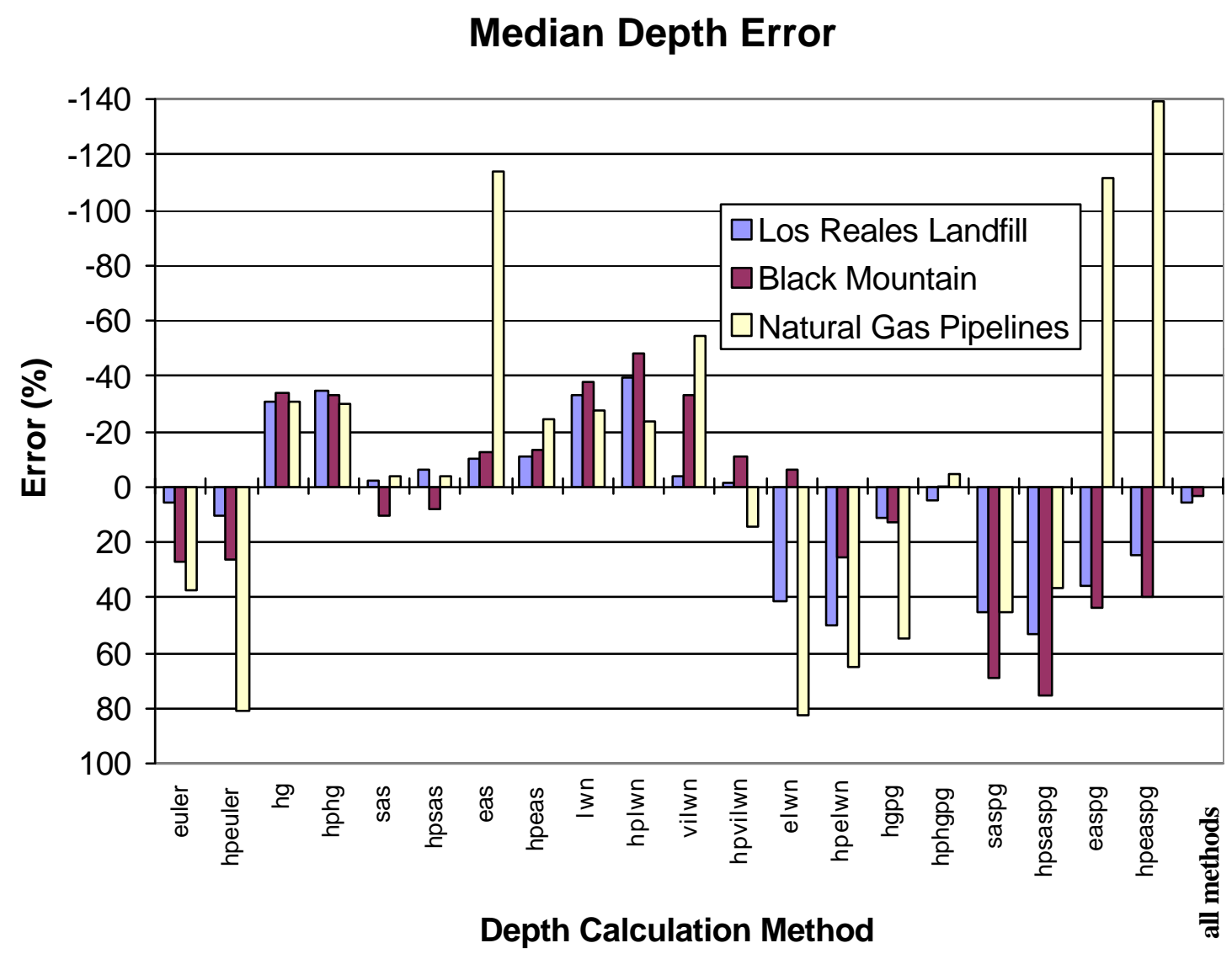

euler - euler deconvolution

hg - horizontal gradient

sas - simple analytic signal

eas - enhanced analytic signal

Iw - local wavenumber

elw - enhanced local wavenumber hp - calculated from high-pass filtered data

pg - calculated from pseudo-gravity data

vi - calculated from the vertical integral

Figure 29. Median depth error for each method over each target. Negative error values represent depths that are too shallow, while positive error values represent depths that are too deep. The step-model enhanced analytic signal depth error is shown for the natural gas pipelines. The Euler depth error for Black Mountain is for a structural index of 1 . For all methods combined, the median error is not shown for the pipelines, since they are considered to be a special case and the error values vary drastically. 


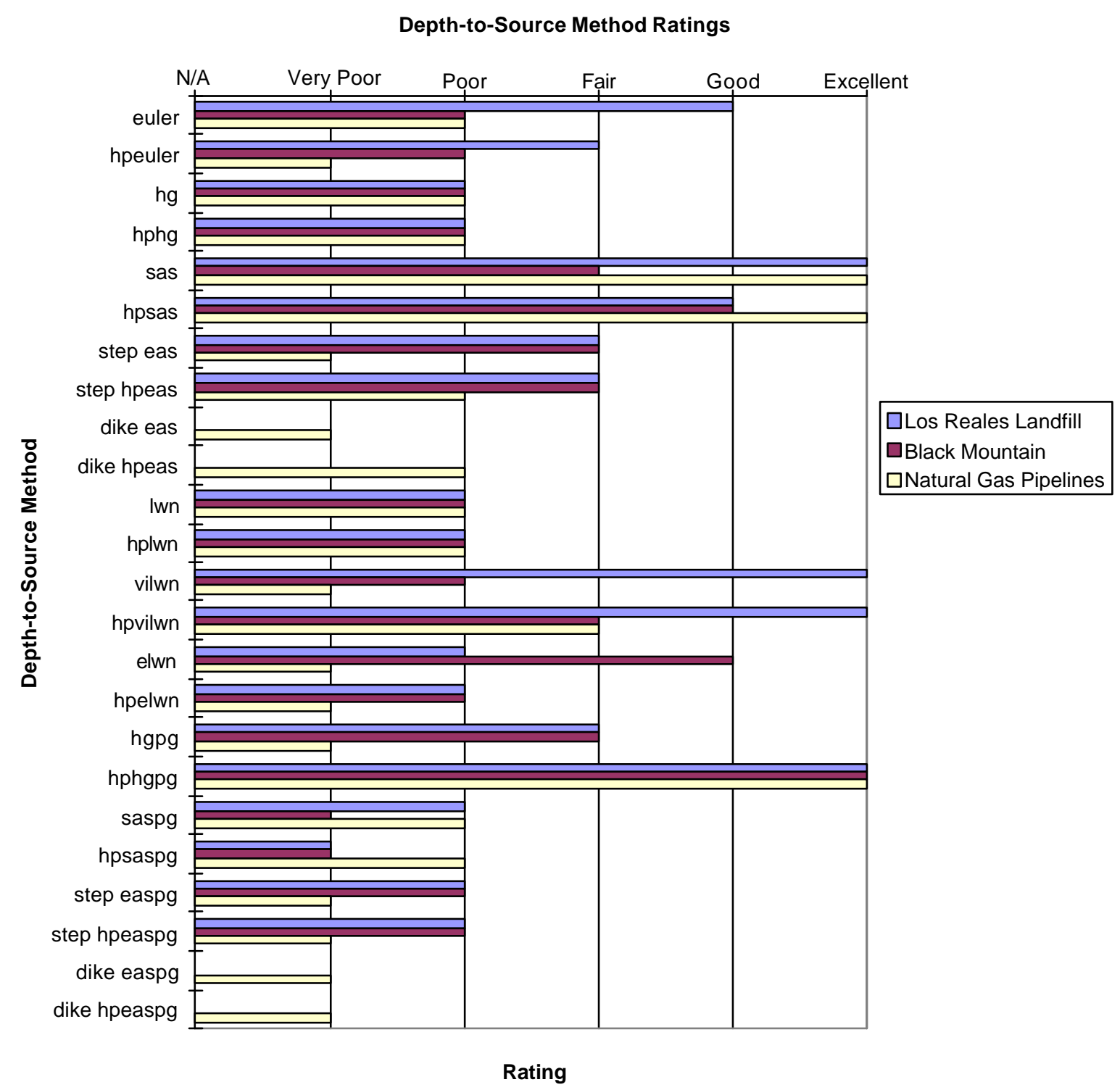

hp - calculated from high-pass filtered data

pg - calculated from pseudo-gravity data

vi - calculated from the vertical integral step - calculated using the infinite step model dike - calculated using the dike model

Figure 30. Depth-to-source me thod ratings. 
Reales Landfill and Black Mountain are considered, the horizontal gradient method applied to the total pseudo-gravity anomaly field and the step model enhanced analytic signal applied to the total and high-pass filtered magnetic anomaly field also yielded favorable results (Figure 30).

Applying a high-pass filter to the data before calculating depths did not provide a consistently better or worse result for all of the methods. The expected result was that of fewer deeper solutions due to the removal of longer wavelength anomalies, and a tightening of the calculated depth distribution (i.e. smaller standard deviation). However, this was not always the case (Figure 13, 17, 21 and Tables 1-3). Only two methods appear to have benefited from this step. The results from the local wavenumber method applied to the vertical integral of the magnetic anomaly field and the horizontal gradient method applied to the pseudo-gravity anomaly field greatly improved from this step over all three test sites. Applying a high-pass filter to the data before calculating the depths via the local wavenumber method decreased the occurrences of shallow solutions and shifted the clustered depth values around a deeper central value. For the horizontal gradient method, filtering decreased the occurrences of deep solutions and shifted the clustered depth values around a shallower central value.

Euler deconvolution only yielded good results over the Los Reales Landfill. Over Black Mountain, a structural index of 1 resulted in calculated depths that were too deep (26\% to $27 \%)$, and a structural index of 0.5 resulted in depths that were too shallow (-20\% to $-21 \%)$. Both indices appeared to give good solution clustering around the topographic expression of Black Mountain (Appendices B7, B8, B20 and B21). This 
illustrates the limitations of the method, in that selecting a proper structural index over complex structures is difficult. Over the natural gas pipelines, a structural index of 2 resulted in calculated depths that were too deep (38\% to $81 \%$ ). Also, there seems to be a bimodal distribution of error values (Figure 21), possible due to the polarity reversal occurring in the pipelines. These results may be due to a poor choice of the structural index and may show this method's lack of effectiveness over sources with a complex magnetization. A structural index of 2 provided good solution clustering over the pipelines (Appendices C7 and C19). However, the anomaly is due the flow of electric current through the pipelines. Without the presence of the Earth's field, the magnetic field due to the pipelines would more closely resemble that of a gravitational field forming concentric circles around a cylindrical geometry (Figure 31). This type of field is not properly described as a line of dipoles, as is assumed by using a structural index of 2. Although Euler deconvolution provides the largest number of solutions, it does not seem to work well when the source body is not a simple, known geometry or where the magnetization is complex.

The horizontal gradient and local wavenumber methods applied to the total and high-passed filtered magnetic anomaly field yielded depth estimates that were too shallow $(-23 \%$ to $-48 \%)$. This was expected, however, as these two methods assume that the source body has infinite thickness. The simple and enhanced analytic signal methods also make this assumption. However, they do not seem to be as sensitive to source body thickness as does the horizontal gradient and local wavenumber methods. When applied to the pseudo-gravity field to attain maximum depth estimates, the simple and enhanced 


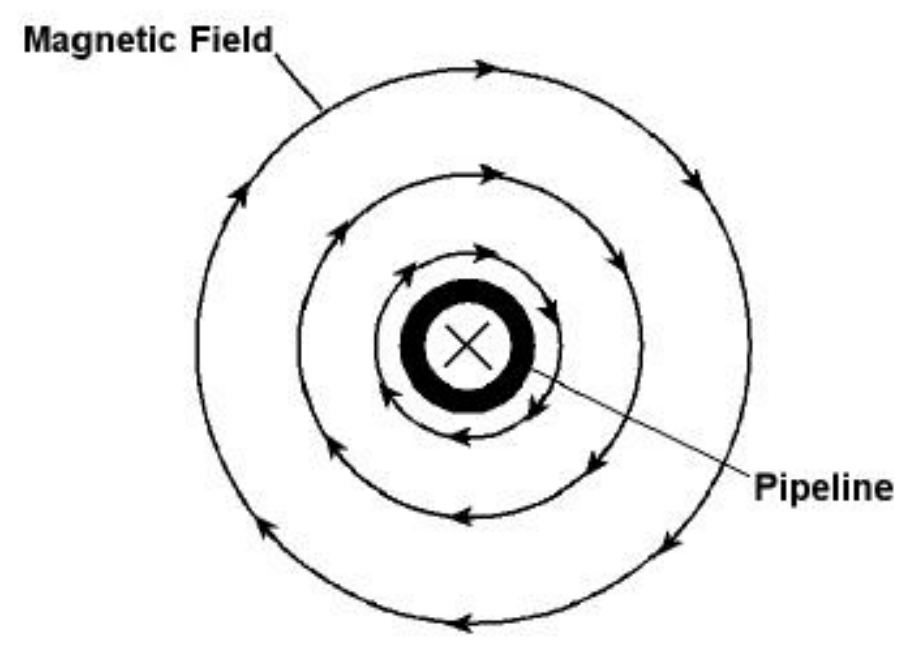

Figure 31. Cross-section of the magnetic field due to a pipeline with cathodic protection. Direction of current flow is into the image.

analytic signal methods yielded solutions that were too deep ( $25 \%$ to $75 \%$ ), with the exception of the enhanced analytic signal method over the natural gas pipelines where the solutions were too shallow $(-58 \%$ to $-139 \%)$. Over Black Mountain, the depths were deeper than the top of the magnetic sources, but because of complex geometry there was no way to verify whether or not these depths accurately depict the bottoms of some of these contacts.

The worst performance came from the enhanced analytic signal method, when assuming a dike model over the natural gas pipelines, and the enhanced local wavenumber method. The enhanced local wavenumber method yielded good results over Black Mountain. However, the standard deviation of error values about the mean is exceptionally high (Table 2). This spread of the occurrence of depth estimates suggests that the median error is not indicative of a converging solution. This is somewhat disappointing considering the method promises proper depth-to-source estimates 
regardless of the direction of magnetization and body geometry. Both methods produced many erroneous solutions, with many depth estimates that resulted in errors mich greater than $\pm 100 \%$. The problem with these methods lies in the sensitivity of the equations used to calculate depth estimates for both methods (Equations 35 and 42). In both methods, the depth to the magnetic contact is dependant upon the inverse of the difference of two values. In all cases, these values were very small and proximal, which led to high computational error. 


\section{GEOLOGIC SITES OF INTEREST}

After evaluating the performance of each method over the test sites, the methods that worked best were applied over geologic sites of interest within the Tucson basin. Two sites that were of particular interest included a suspected occurrence of buried volcanics underneath the Los Reales Landfill and magnetic anomalies near the Mission Mine area (Figure 32) supporting the existence of a low-angle detachment fault. The simple analytic signal method, the local wavenumber method and the horizontal gradient method were used to determine the depths to the magnetic sources. The enhanced analytic signal method was not used, however, since it did not perform as well as the other methods due to its susceptibility to noise. 


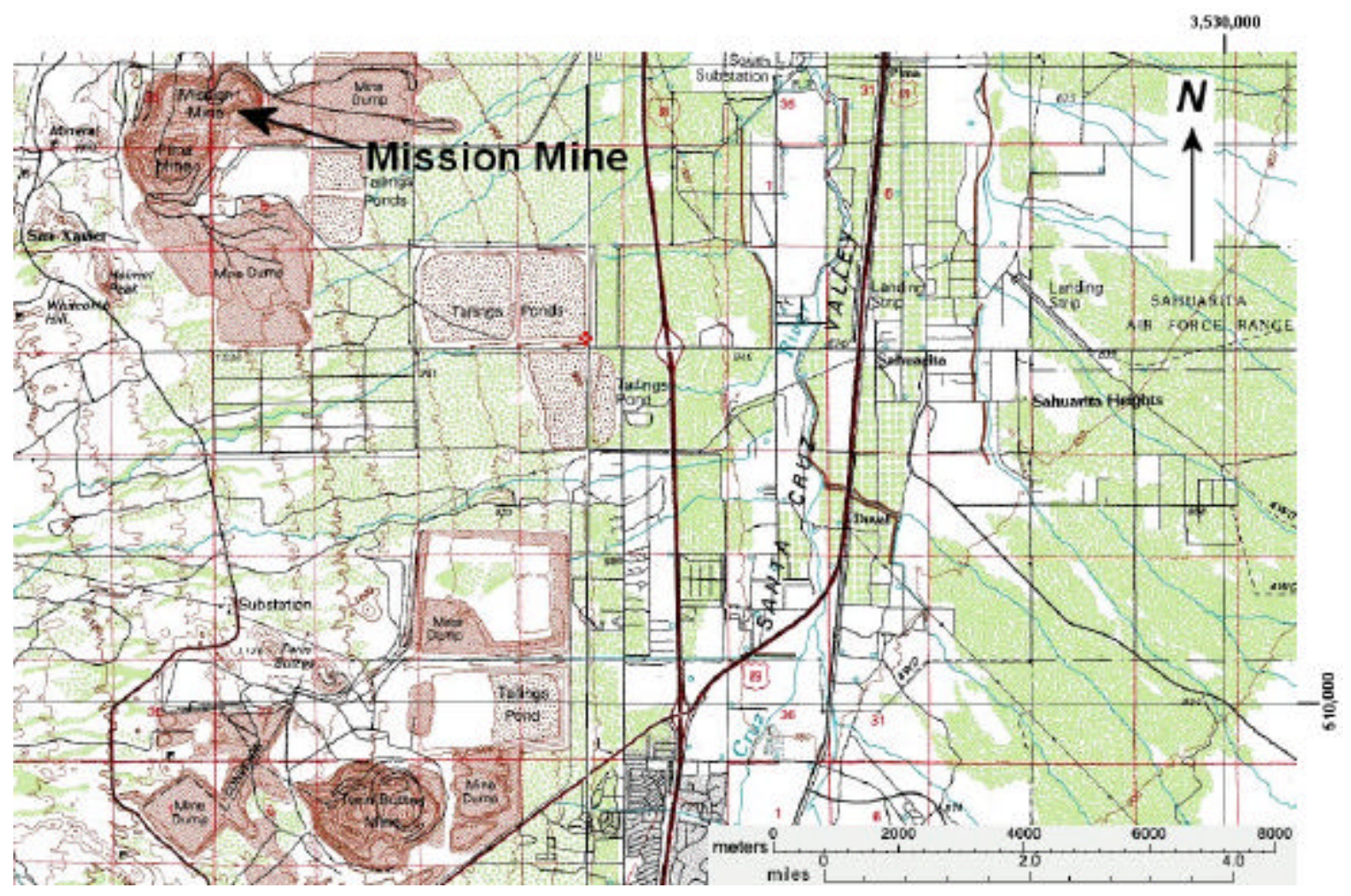

Figure 32. Location of the Mission Mine area (modified from TopoZone). 


\section{Buried Volcanics}

Upon applying a high-pass Butterworth filter, using an $8^{\text {h }}$ degree function and a cutoff wavelength of 2500 meters/cycle, to the gridded magnetic anomaly data over the landfill, a deeper, broader magnetic anomaly was discovered. The anomaly is reversed with a declination of about 180 degrees and lies directly beneath the landfill (Figure 33). Closer examination of the area surrounding the landfill revealed several reversed magnetic anomalies that run across the area west of Tucson and into southern Tucson (Figure 34). These anomalies are presumed $\mathbf{b}$ be the result of a sequence of now buried Tertiary volcanics, similar to those that comprise Black Mountain.

The reduced magnetometer data were gridded with a 10-meter grid interval using the bi-directional line gridding algorithm in OASISmontaj. In order to isolate the reversed anomaly and to reduce any interference effects from deeper or shallower sources, a band-pass filter was applied to the data. Before any derivatives were calculated, a 3000-meter high-pass and a 500-meter low-pass Butterworth filter, using an $8^{\text {th }}$ degree function, were applied to the gridded data. All signals were subsequently calculated from the band-pass filtered 10-meter grid.

The simple analytic signal method did not yield any depths around the perimeter of the postulated buried volcanics due to the interference from the overlying landfill (Figure 35). For the remaining methods, the depth below ground surface was determined by subtracting sampled radar altimeter grid data from the calculated depths. The grids were re-gridded at 80 meters to find the magnetic contacts. Before re-gridding, 


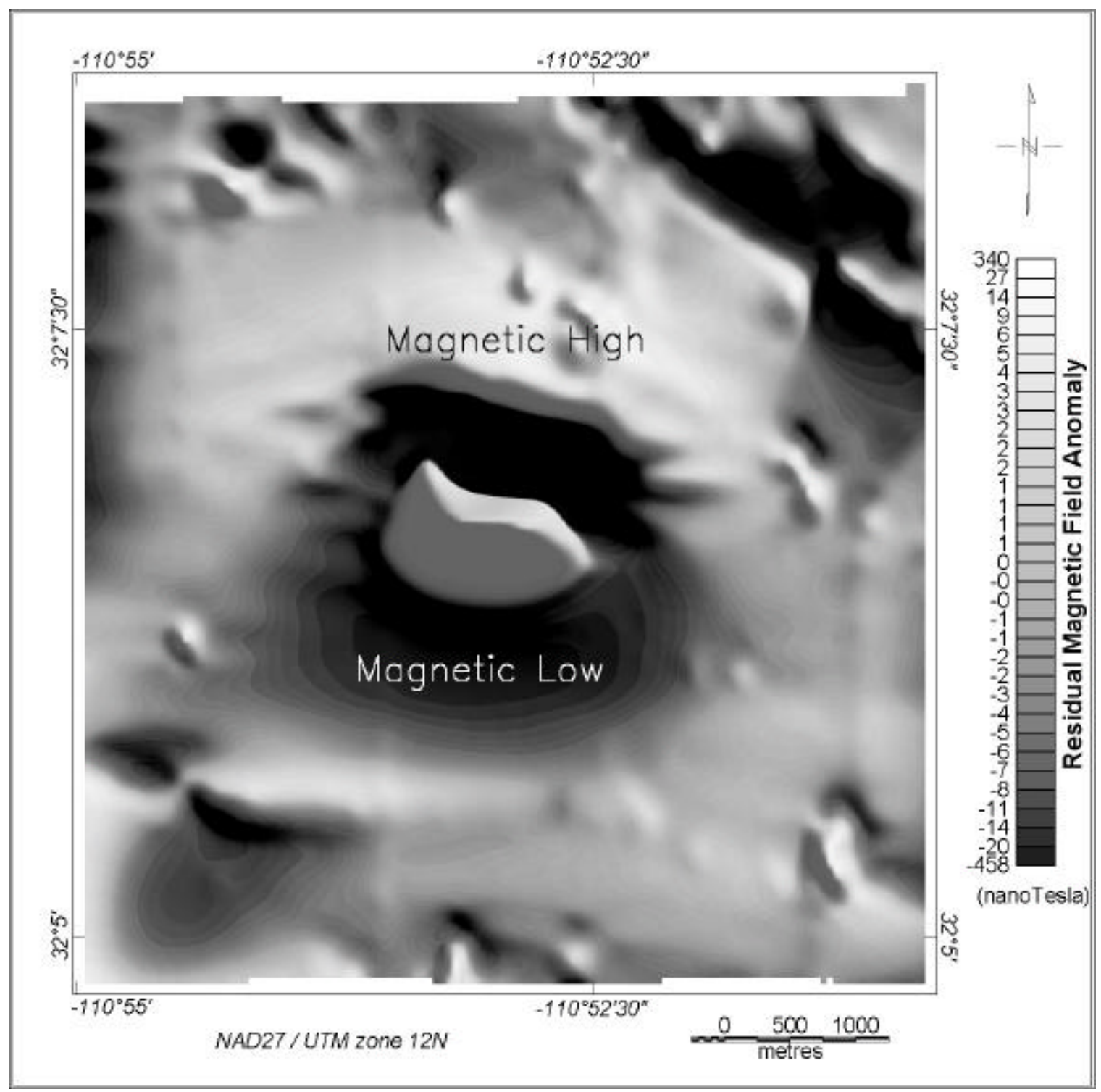

Figure 33. High-pass filtered magnetic field anomaly map showing an apparent reversed anomaly beneath the Los Reales Landfill. 


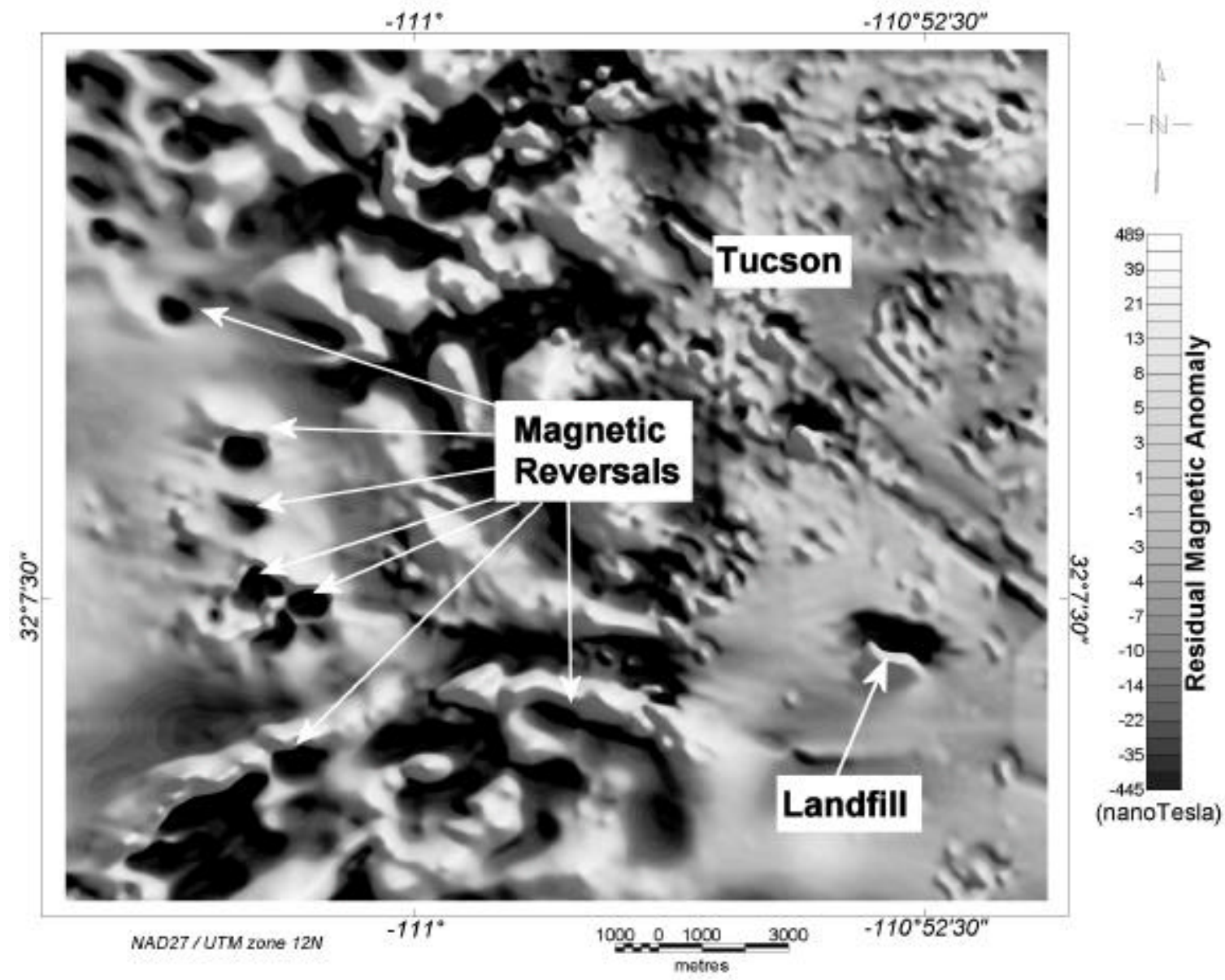

Figure 34. Map showing reversed anomalies just west of Tucson. There are a number of reversed anomalies in this map that have not been labeled. A band-pass filter has been applied to the magnetic data. 


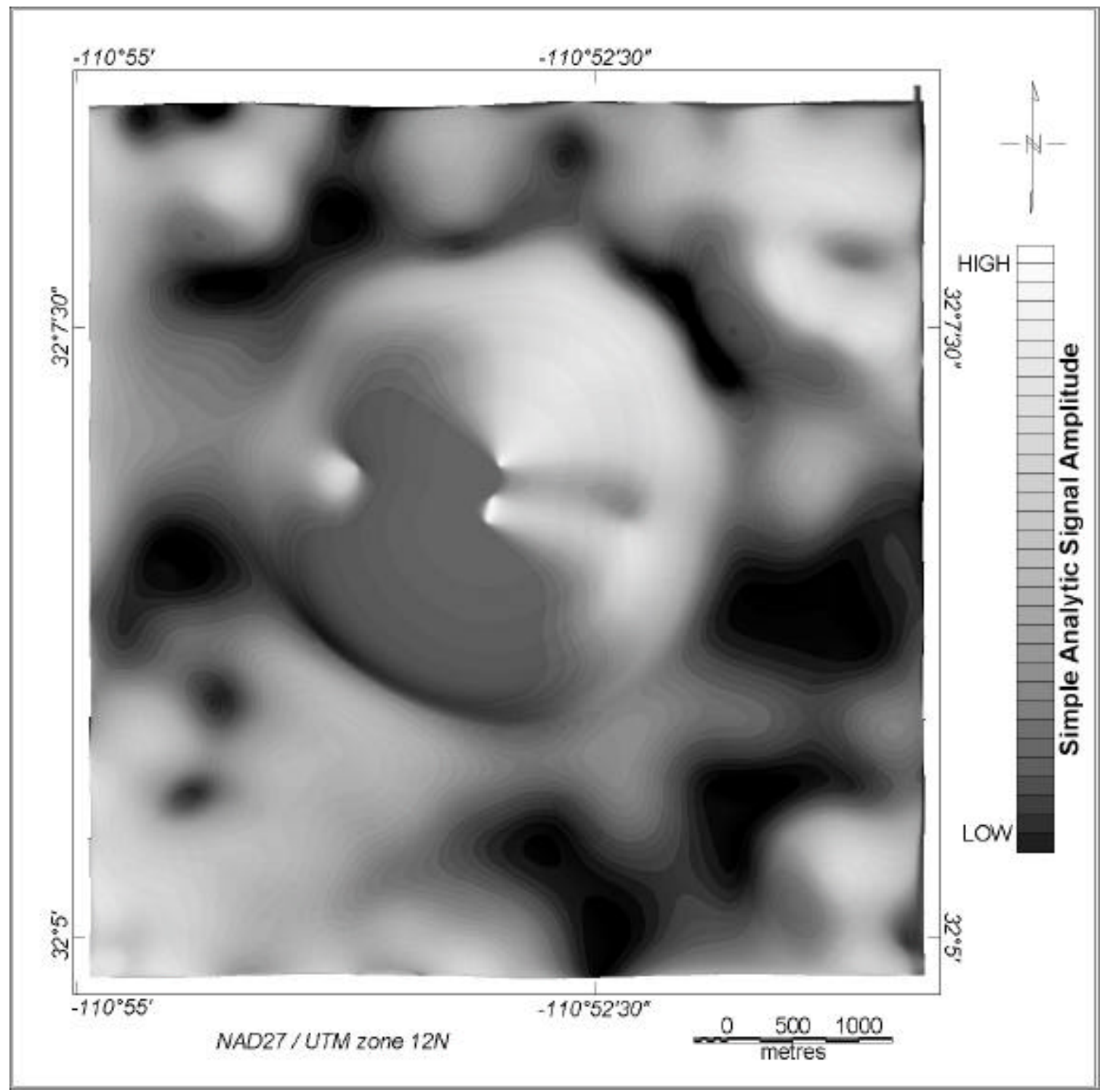

Figure 35. Map of the simple analytic signal amplitude over the suspected buried volcanics. There is interference from the analytic signal amplitude of the overlying landfill. 
a low-pass linear convolution filter with a length of 80 meters was used in the direction of the grid lines.

Table 4 summarizes the depth-to-source error results for each method used. Figure 36 shows the depth-to-source histograms for all of these methods. Figures 37 and 38 are maps of the local wavenumber of the vertical integral of the magnetic anomaly field and the horizontal gradient magnitude of the pseudo-gravity field, respectively, with plots of the calculated strikes and depths.

Table 4. Depth-to-source statistics for the suspected buried volcanics. Values represent depths below ground level.

\begin{tabular}{|c|c|c|c|c|c|c|c|c|c|}
\hline Method & Median & Mean & Standard Dev. & Kurtosis & Skewness & Range & Minimum & Maximum & Count \\
\hline vilwn & 189.4 & 196.6 & 77.9 & -0.8 & 0.1 & 292.4 & 55.5 & 347.9 & 50 \\
\hline hgpg & 150.0 & 149.4 & 70.1 & -1.0 & 0.2 & 259.8 & 27.3 & 287.1 & 53 \\
\hline \multicolumn{10}{|c|}{$\begin{array}{l}\text { Average Median } \\
169.7\end{array}$} \\
\hline $\begin{array}{ll}\text { hg } & -\mathrm{h} \\
\text { Iwn } & - \text { lo }\end{array}$ & $\begin{array}{l}\text { rizontal gr } \\
\text { al wavenu }\end{array}$ & $\begin{array}{l}\text { adient } \\
\text { Imber }\end{array}$ & & $\begin{array}{ll}\text { pg } & - \text { calcul } \\
\text { vi } & \text { - calculc }\end{array}$ & $\begin{array}{l}\text { ed from pseu } \\
\text { ed from the } v\end{array}$ & $\begin{array}{l}\text { o-gravity } \\
\text { rtical inte }\end{array}$ & $\begin{array}{l}\text { data } \\
\text { gral }\end{array}$ & & \\
\hline
\end{tabular}


Figure 36. Depth-to-source histograms for each method over the buried volcanics(?). Values represent depths below ground level.
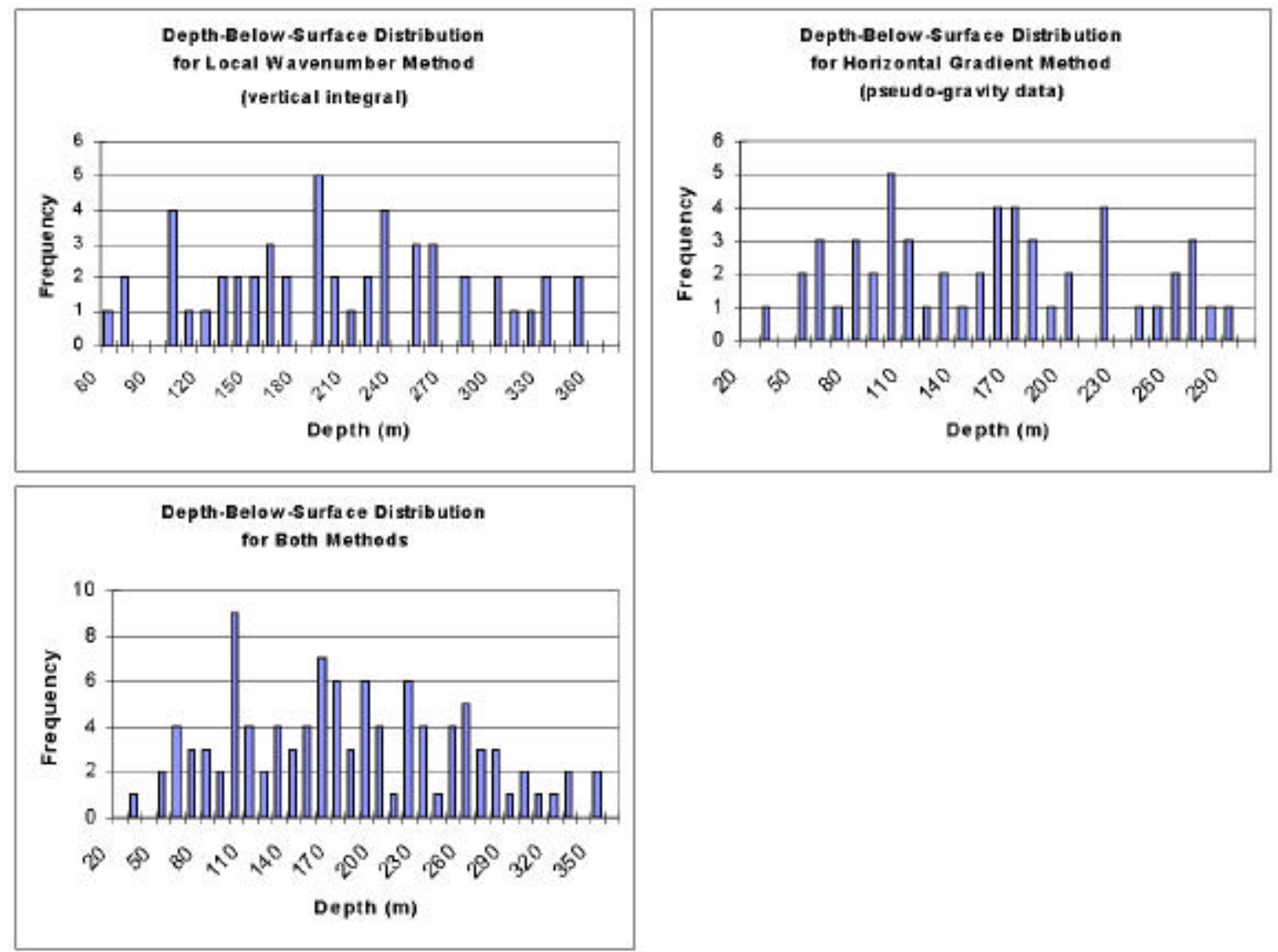


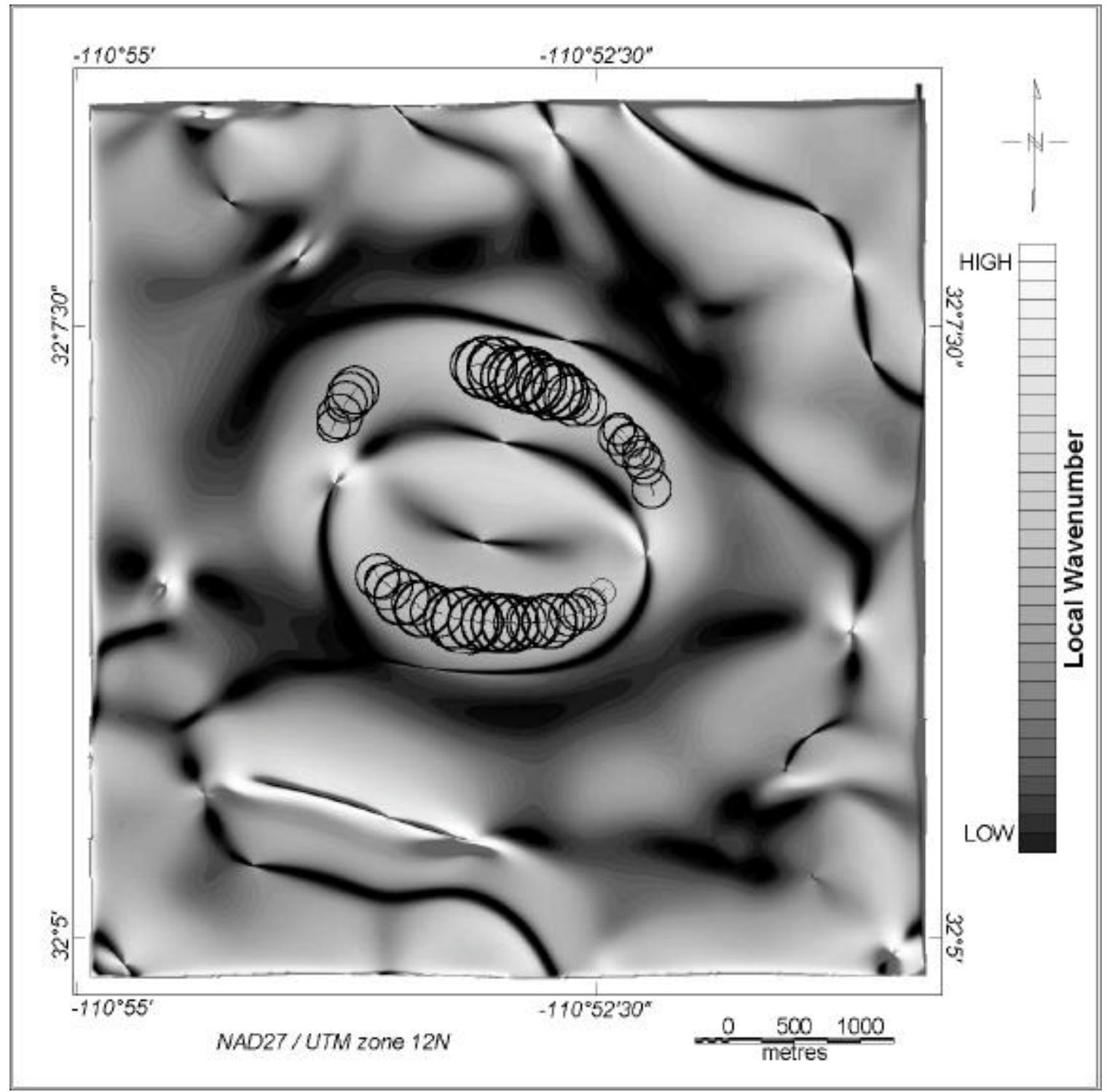

Figure 37. Depth-to-source map over the suspected buried volcanics using the local wavenumber method applied to the vertical integral of the total magnetic field anomaly. 


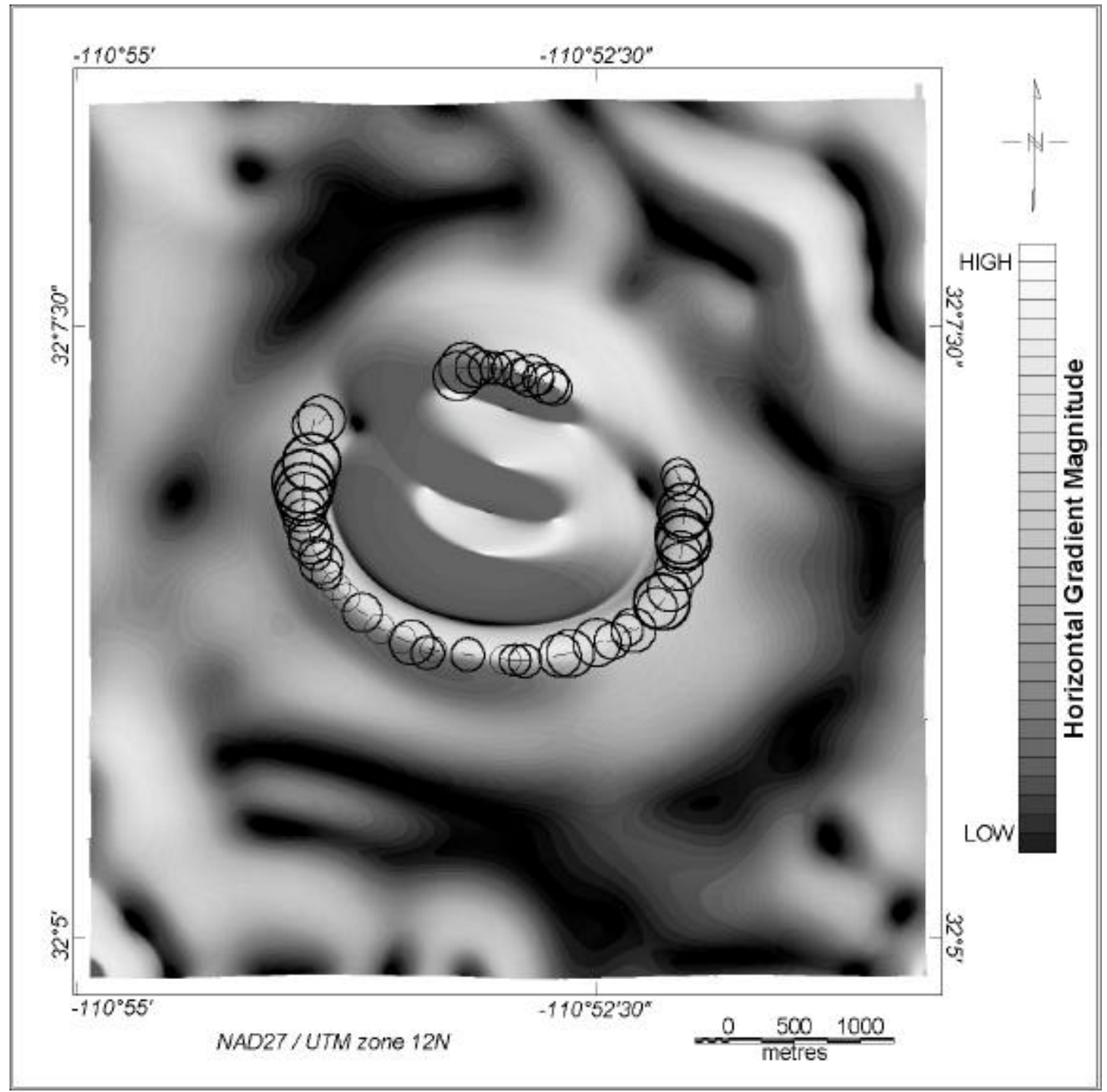

Figure 38. Depth-to-source map over the suspected buried volcanics using the horizontal gradient method applied to the pseudo-gravity anomaly field. 
The average median depth estimate suggests that the postulated volcanics are buried nearly 170 meters beneath the Los Reales Landfill. Assuming there was a constant flight elevation of 152 meters and the average median depth estimate represents the correct depth to the magnetic source, the median depth estimates from both methods are within $12 \%$ of the total depth-to-source from one another.

It should be noted that there are two other possible explanations for the reversed anomaly beneath the landfill. The regional field surrounding the landfill indicates the possibility of a magnetic "hole" in the area. Two truncated blocks of magnetic material, one just to the north and the other just to the south of the landfill, would also appear as a reversed anomaly, assuming an induced magnetization in both blocks. The southern block would reveal its south magnetic pole, while the northern block would reveal its north magnetic pole. Such a scenario would occur if the landfill were overlying a graben that formed during the north-northwest extension that was responsible for the formation of the basin and range. Another possible explanation for the reversed anomaly is that it is nothing more than an artifact of filtering. The anomaly that surrounds the landfill could be due to Gibb's phenomena (ringing in the data), since the amplitude of the landfill anomaly is considerably large. However, several filtering parameters were used to test this hypothesis, and it was determined that the high-pass filter that was used has a smooth enough function to reduce or eliminate the occurrence of such artifacts. 


\section{Detachment Fault}

The total-field magnetic anomaly data just east of the Mission Mine area (Figure 32) suggest the existence of a low-angle fault. Surface expressions of such faults lie a few kilometers west and southwest of the Mission mine area (Drewes, 1980). A distinct linear anomaly accompanied by a somewhat rounded dipole anomaly seems to be mirrored to the northeast, with an offset of approximately 6 kilometers between the centers of the magnetic highs in the two rounded anomalies (Figure 39). The anomalies in the northeast are somewhat broader than those in the southwest, which implies their source bodies are at greater depth. Given the geologic history of the Tucson basin, the anomalies may be due to an intrusive body that has been cut by a low-angle detachment fault, with the northeastern block sliding to greater depth with respect to the southwestern block. Attaining depths to the magnetic source bodies in the northeast and southwest will provide a clue to the amount of fault movement that may have occurred in the area.

The reduced magnetometer data were gridded with a 10-meter grid interval using the bi-directional line gridding algorithm in OASISmontaj. In order to isolate the anomalies of interest and to reduce any interference effects from deeper or shallower sources, a band-pass filter was applied to the data. Before any derivatives were calculated, an 8000-meter high-pass and a 500-meter low-pass Butterworth filter, using an $8^{\text {th }}$ degree function, were applied to the gridded data. All signals were subsequently calculated from the band-pass filtered 10-meter grid. 


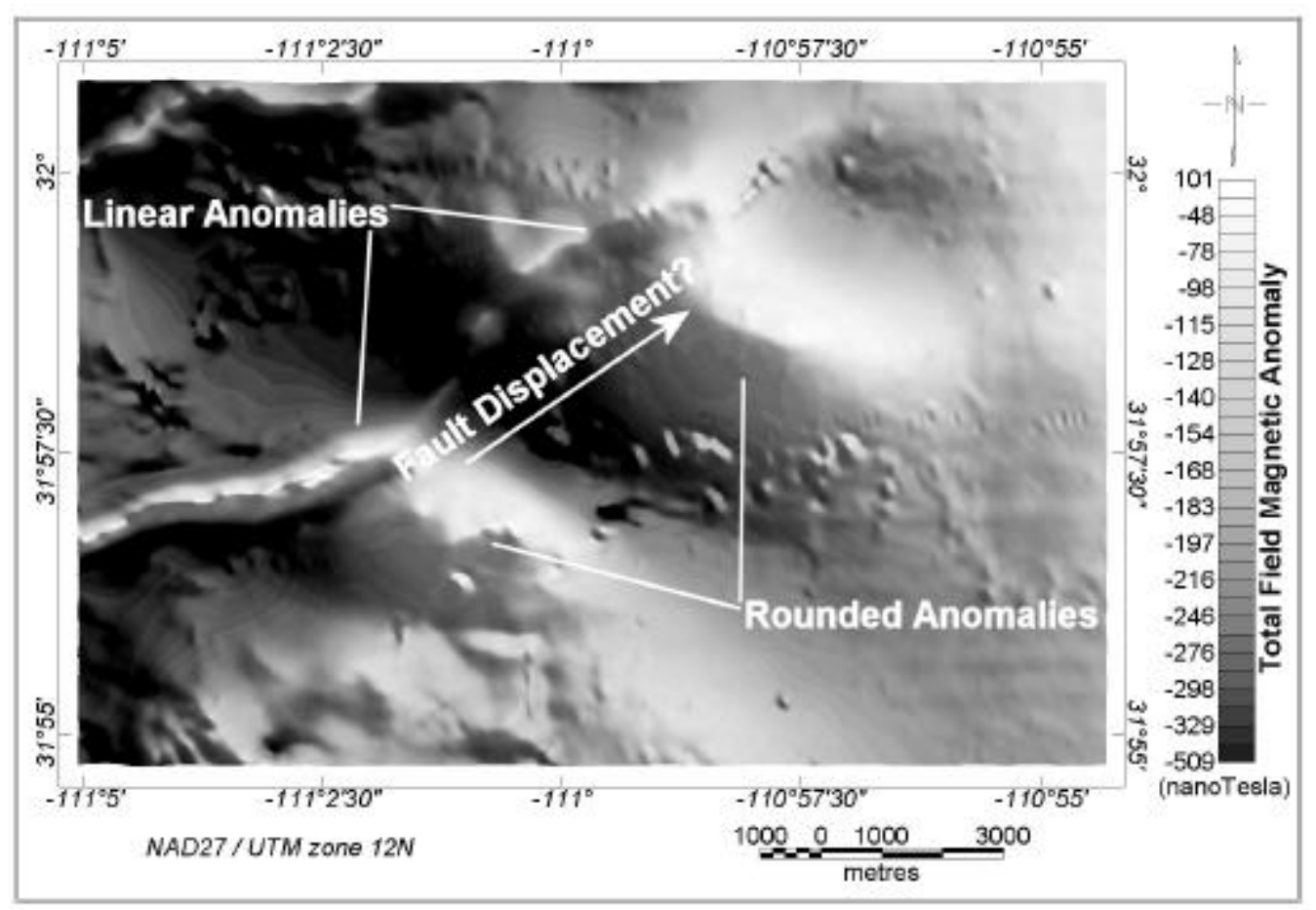

Figure 39. Total field magnetic anomaly map showing the apparent offset of similar magnetic anomalies east of the Mission Mine area.

The depth estimates over the rounded dipole anomalies in the northeast and the southwest were analyzed separately. The depth below ground surface was determined by subtracting sampled radar altimeter grid data from the calculated depths. The grids were re-gridded at 80 meters to find the magnetic contacts. Before re-gridding, a low-pass linear convolution filter with a length of 80 meters was used in the direction of the grid lines.

Table 5 summarizes the depth-to-source results for each method used. Figure 40 shows the depth-to-source error histograms for all of these methods. Figures 41 through 44 are the specific signal used for each calculation with the relevant calculated strikes and depths plotted as lines and circles, respectively. 
Table 5. Depth-to-source statistics for the detachment fault. Values represent depths below ground level. The top table represents the shallow anomaly to the southwest, while the bottom table represents the deeper anomaly to the northeast. The "Average Median" does not take into account the results from the horizontal gradient method applied to the pseudo-gravity field.

\begin{tabular}{|c|c|c|c|c|c|c|c|c|c|}
\hline Method & Median & Mean & Standard Dev. & Kurtosis & Skewness & Range & Minimum & Maximum & Count \\
\hline sas & 281.0 & 280.0 & 77.0 & -0.9 & -0.3 & 275.0 & 117.6 & 392.6 & 30 \\
\hline vilwn & 263.5 & 249.7 & 105.9 & 1.6 & -0.6 & 580.0 & -41.7 & 538.3 & 45 \\
\hline hgpg & 641.7 & 581.6 & 213.3 & -1.6 & 0.1 & 630.9 & 299.4 & 930.4 & 46 \\
\hline hg & 216.5 & 224.1 & 67.2 & -0.2 & 0.5 & 302.7 & 82.8 & 385.5 & 61 \\
\hline \multicolumn{10}{|c|}{$\begin{array}{l}\text { Average Median } \\
\quad 253.7\end{array}$} \\
\hline \multicolumn{4}{|c|}{$\begin{array}{ll}\text { hg } & \text { - horizontal gradient } \\
\text { sas } & \text { - simple analytic signal } \\
\text { Iwn } & \text { - local wavenumber }\end{array}$} & \multicolumn{6}{|c|}{$\begin{array}{l}\text { pg - calculated from pseudo-gravity data } \\
\text { vi } \quad \text { - calculated from the vertical integral }\end{array}$} \\
\hline \multicolumn{10}{|c|}{ (meters) } \\
\hline Method & Median & Mean & Standard Dev. & Kurtosis & Skewness & Range & Minimum & Maximum & Count \\
\hline sas & 386.8 & 392.7 & 178.7 & -0.1 & 0.4 & 707.9 & 69.5 & 777.4 & 38 \\
\hline vilwn & 351.5 & 361.9 & 189.5 & -0.5 & 0.3 & 760.4 & 20.2 & 780.7 & 68 \\
\hline hgpg & 911.1 & 836.7 & 257.2 & 1.4 & -1.6 & 877.1 & 245.5 & 1122.6 & 27 \\
\hline hg & 379.6 & 365.9 & 188.9 & 0.8 & 0.4 & 921.3 & 19.3 & 940.6 & 92 \\
\hline
\end{tabular}


Figure 40. Depth-to-source histograms for each method over the detachment fault. Values represent depths below ground level.
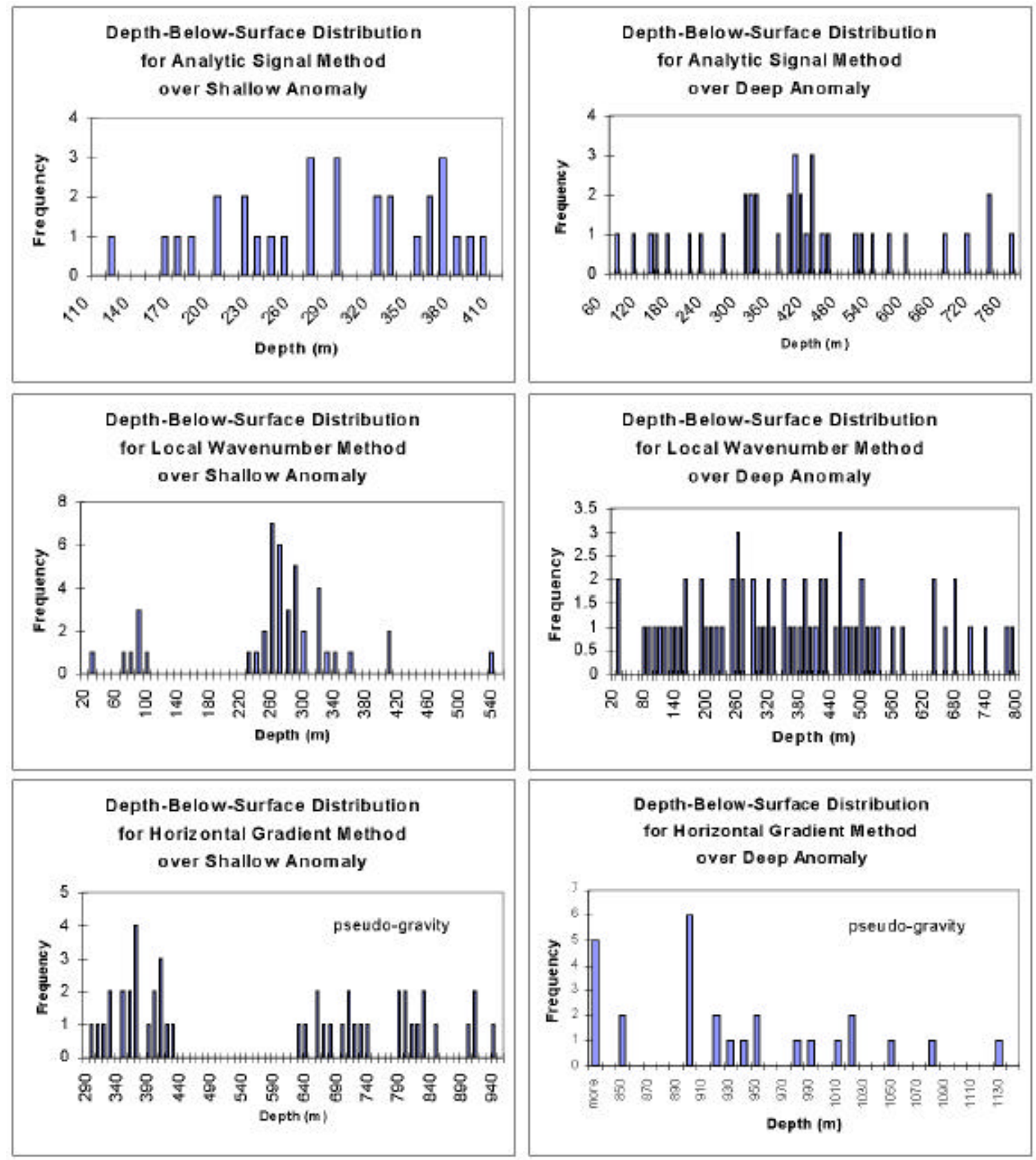
Figure 40. cont...
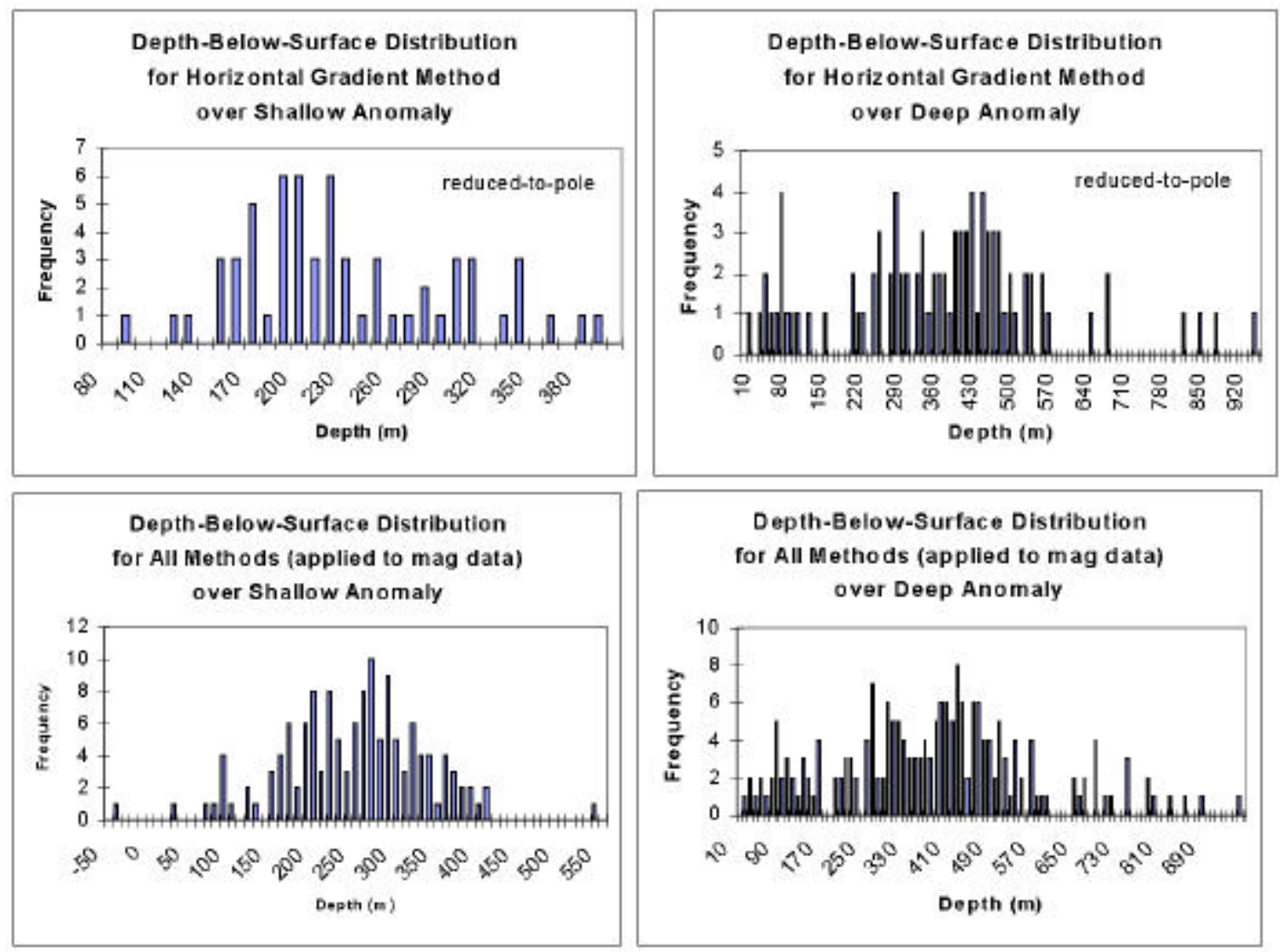


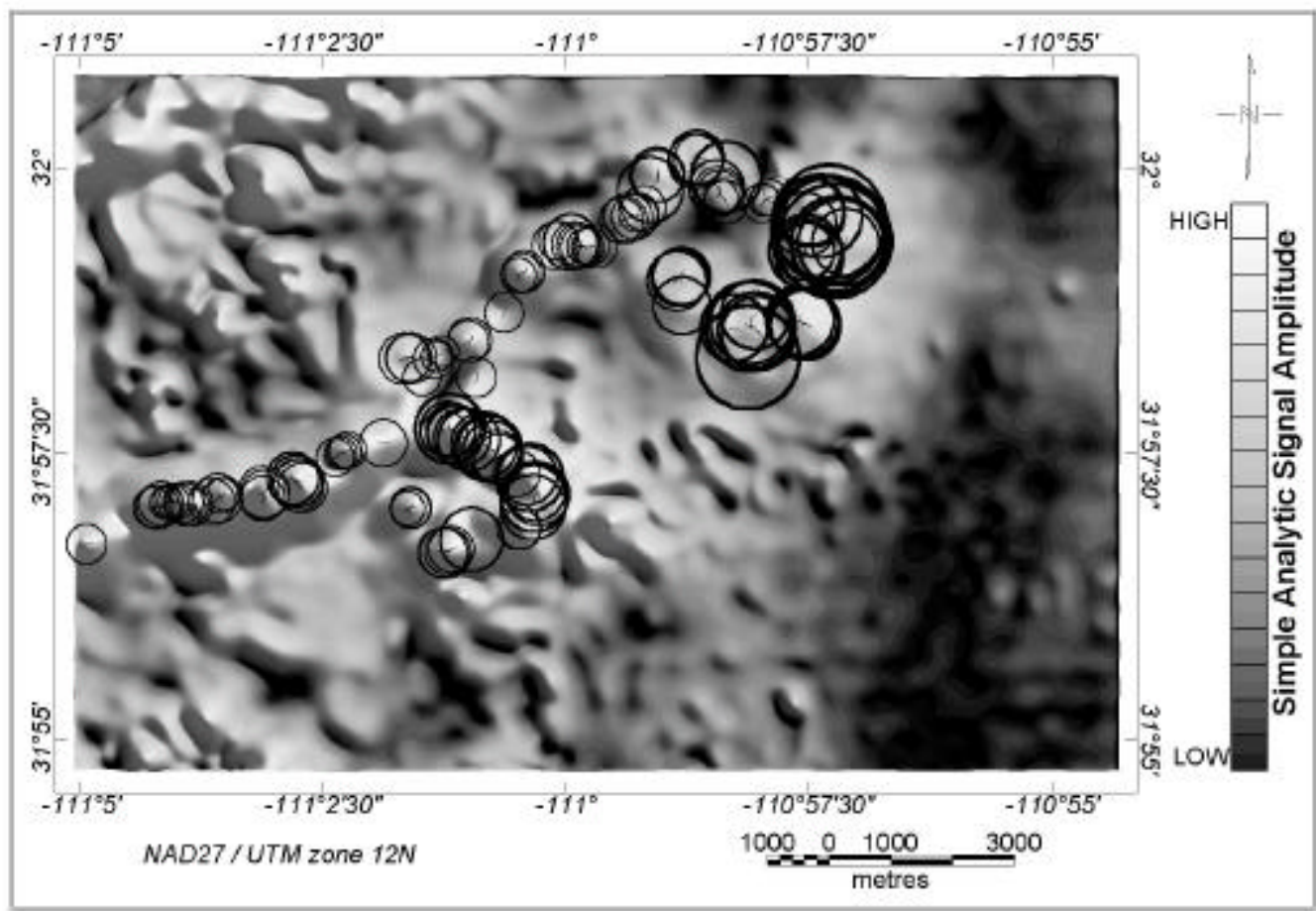

Figure 41. Depth-to-source map over the detachment fault using the simple analytic signal method. 


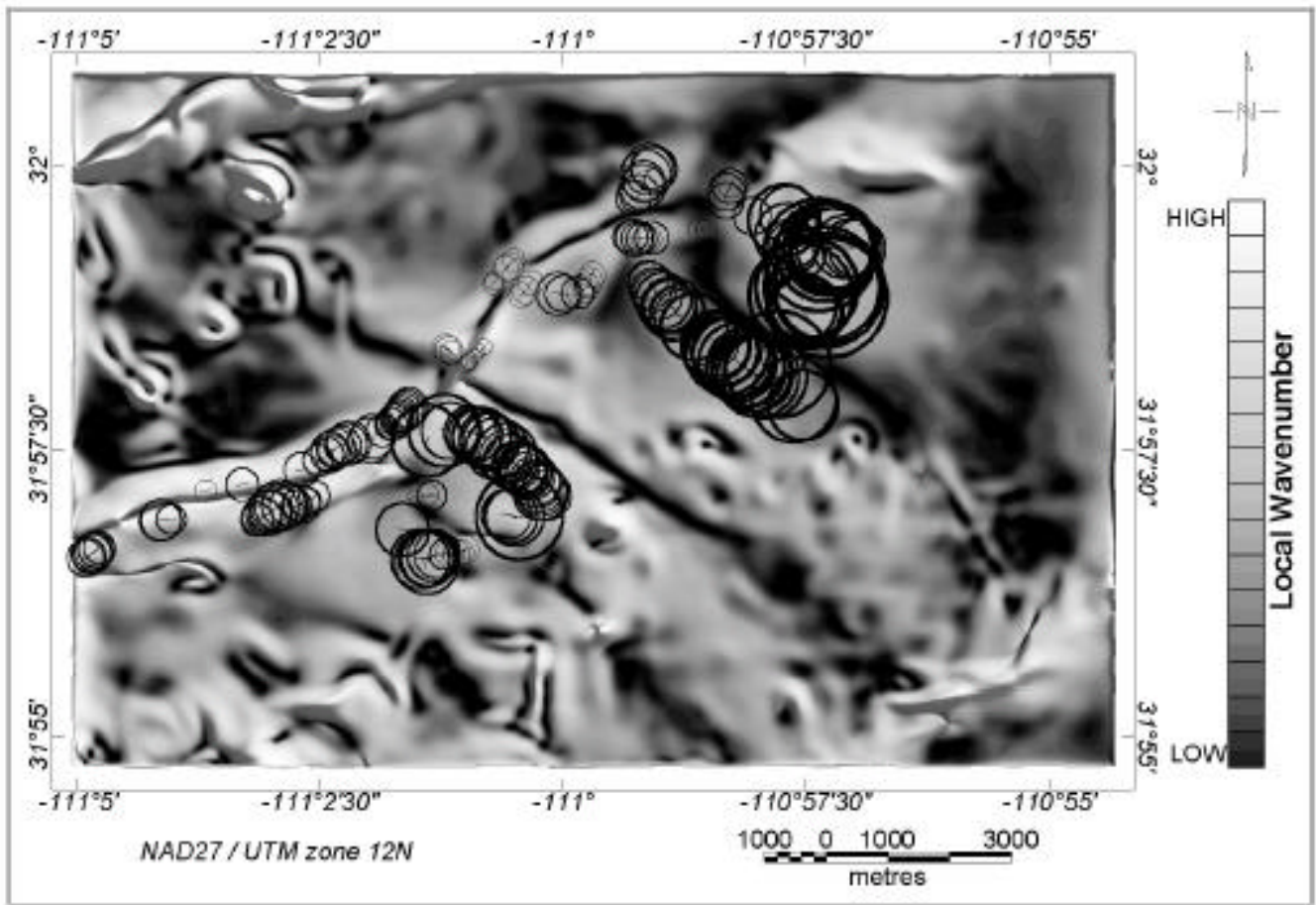

Figure 42. Depth-to-source map over the detachment fault using the local wavenumber method applied to the vertical integral of the total magnetic field anomaly. 


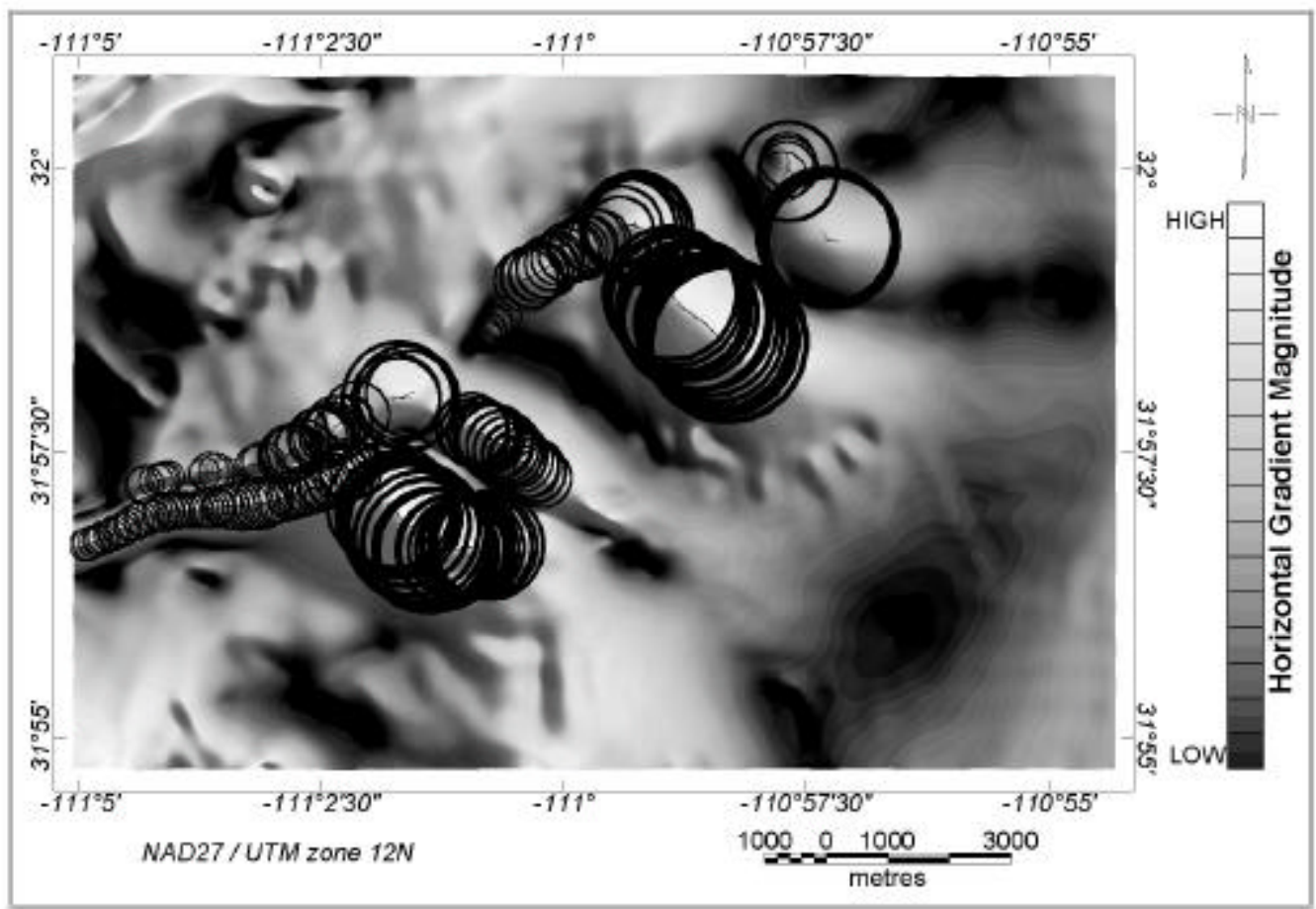

Figure 43. Depth-to-source map over the detachment fault using the horizontal gradient method applied to the pseudo-gravity anomaly field. 


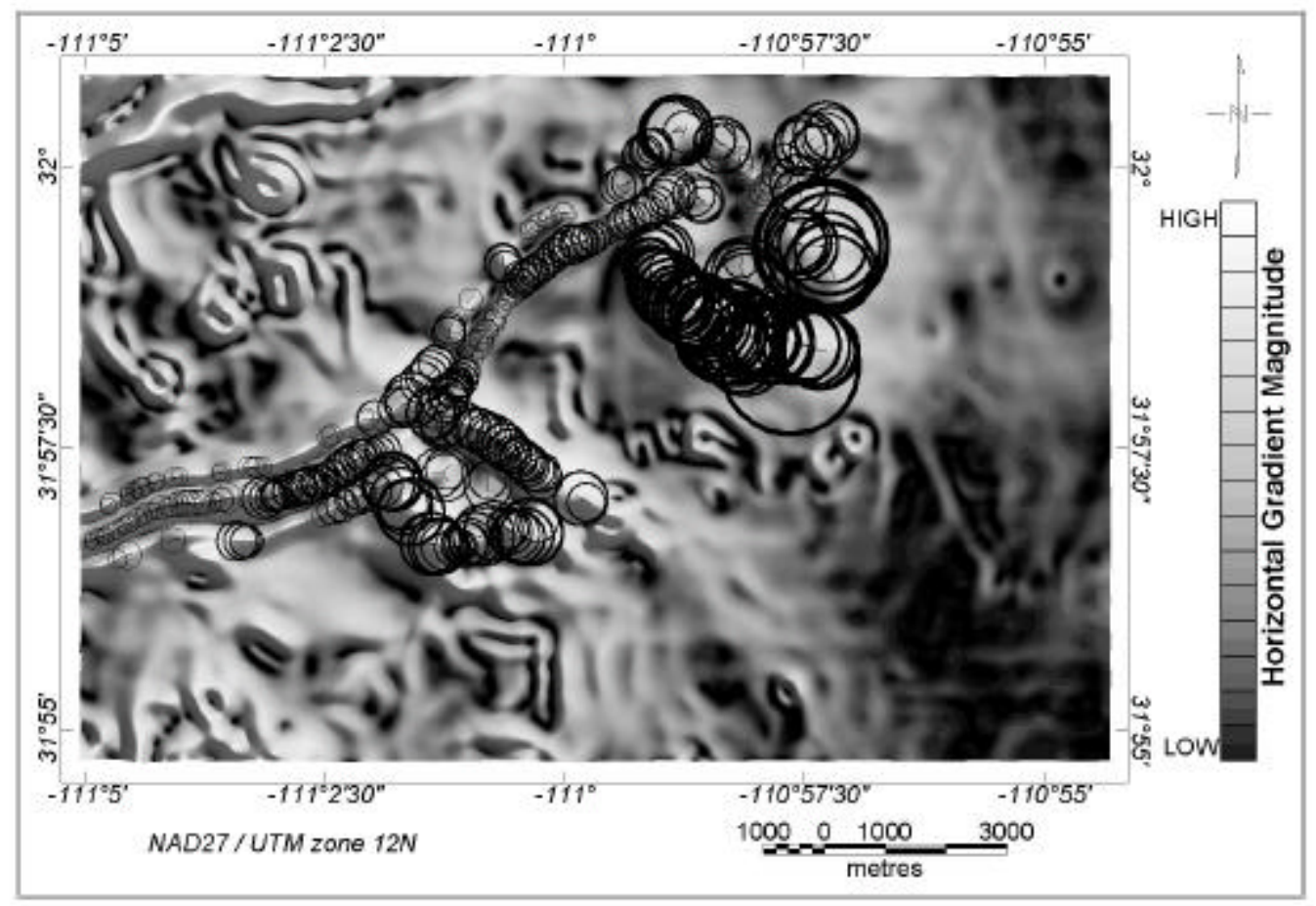

Figure 44. Depth-to-source map over the detachment fault using the horizontal gradient method applied to the reduced-to-pole magnetic anomaly field.

The horizontal gradient method applied to the pseudo-gravity field yielded depth estimates that were much deeper than those by the simple analytic signal and local wavenumber methods (Figures 41 through 43). Since the horizontal gradient method was applied to the pseudo-gravity field, the depth estimates represent maximum depths with the assumption of thin sources. The large difference in depth estimates suggests that the source bodies are rather thick. The anomalies are most likely due to faulted igneous intrusions that initially extended deep within the crust. To compare minimum with maximum depth estimates, the horizontal gradient method was also applied to the reduced-to-pole magnetic anomaly field (Figure 44 and Table 5). The resulting depth 
estimates were closer to those from the other methods. It may be possible to determine whether or not a source body is significantly thick just by comparing the depth estimates obtained by using the simple analytic signal method with those obtained by using the horizontal gradient method applied to the pseudo-gravity field. A large spread between the two sets of depth estimates seems to indicate significantly thick sources. In these cases, the horizontal gradient method applied to the reduced-to-pole magnetic anomaly data would produce depth estimates closer to the true values, since the depth estimates using this approach represent minimum depths to thick sources.

Since the sources are assumed thick, the maximum depth estimates obtained using the horizontal gradient method were not included in the final analysis. The average median depth estimate suggests that the tops of the intrusive bodies are offset by nearly 119 meters. The top of the source body in the southwestern block is approximately 254 meters below the surface, and the top of the source body in the northeastern block is approximately 373 meters below the surface. Assuming there was a constant flight elevation of 152 meters and the average median depth estimate represents the correct depth to the magnetic source, the extreme median depth estimates from all three methods over the southwestern block are within $16 \%$ of the total depth-to-source from one another. Over the northeastern block, this spread is under $7 \%$.

A surface expression of a fault exists approximately 7 kilometers southwest of the shallow rounded anomaly, where Proterozoic rocks lie adjacent to Tertiary rocks (Drewes, 1980). If this fault is responsible for the two offset anomalies, a depth of 254 meters to the top of the source body causing the southwestern anomaly gives a fault dip 
angle of approximately 2.1 degrees. This dip angle is exceptionally small (and unlikely), and does not match the dip angles of other surface faults in the area. Also, a dip angle of 2.1 degrees would give a thickness of 104 meters for the source body causing the rounded anomaly to the northeast. This thickness results in a depth to the bottom of the source body that is only about $20 \%$ deeper than the determined depth to the top of the source (as measured from a flight height of $152 \mathrm{~m}$ ). The depth estimates from the horizontal gradient method applied to the pseudo-gravity field suggest that the source body is much thicker than this. A more likely scenario is a buried fault closer to the rounded anomaly to the southwest, with a dip angle that is much greater than 2.1 degrees. A fault nearly 3 kilometers west of Mission Mine has a dip angle of 21 degrees (Drewes, 1980). Assuming the fault responsible for the offset rounded anomalies is buried just beneath the surface (i.e. effectively at ground level) and the dip angle is also 21 degrees, the surface fault scarp would lie approximately 662 meters southwest of the center of the rounded southwestern anomaly and the thickness of the source body responsible for the northeastern anomaly would be approximately 2,184 meters. This thickness yields a depth to the bottom of the source body that is more than five times the depth to the top of the source, which is consistent with the results from the horizontal gradient method. The throw of such a fault is 2,303 meters. Since this theoretical fault is buried at some depth, and the exact dip angle of the fault is unknown, the throw of the fault could be considerably higher or lower than this value. 


\section{CONCLUSIONS}

\section{Depth Calculation Guidelines}

After much trial and error and testing various methods over targets in the Tucson basin, several guidelines have been devised to aid in the calculation and interpretation of three-dimensional depth-to-source estimates using automated techniques on gridded potential-field data. In particular, these guidelines apply to calculating depths to shallow sources using magnetic data. Following these simple rules will help to reduce the occurrence of false depth estimates:

1. Eliminate sampling bias and high-frequency data not of interest by applying a low-pass filter to the data. This will prevent much unwanted noise when calculating derivatives. An upward continuation is not a good idea, however, because too much anomaly resolution is lost.

2. Apply a high-pass filter to the data when interference from deeper sources is suspected. Applying a high-pass filter over the test sites did not always improve the results, but the drawbacks were small and far outweighed by the benefits.

3. Calculate the derivatives at a small enough grid spacing to encompass the anomaly curvature. A spacing less than one percent of the anomaly wavelength is a good choice. This will prevent noise due to problems arising from using a finite difference algorithm to calculate derivatives. Also, at small grid spacings, any noise introduced when calculating derivatives will be relatively high frequency, 
low amplitude in nature and can be easily removed before finding peaks in the signal of interest.

4. Remove the noise in the derived signal grids by applying a low-pass filter in the grid-line directions. This allows for a smoother grid and a better chance of finding the correct peak values.

5. Re-grid the derived signal grids at a spacing that enables the search window to encompass the peak values without covering more than one peak at a time. This can be accomplished by visually examining the width of the gridded signal peaks of interest. If a poor choice of grid spacing and associated window size is used, too few or too many solutions will result.

6. The simple analytic signal method, the local wavenumber method applied to the vertical integral of the magnetic field, and the horizontal gradient method applied to the pseudo-gravity field (for thin sources) should be used to determine the depth to shallow magnetic sources. If the sources are thick, the horizontal gradient method applied to the reduced-to-pole magnetic data rather than to the pseudo-gravity field will provide better solutions. The enhanced analytic signal should be used with caution and only when you can't resolve the source edges due to interference effects, since it requires calculating high-order derivatives and is quite noisy. Also, it is important to remember that the contact locations determined by the horizontal gradient method will be offset if there is any remanent magnetization. 
Although these rules were derived from calculations made with gridded data, they may also be applied to profile data. The advantage of gridded data over profile data, of course, lies in the fact that a profile can be chosen in any direction over a specified target. It should also be noted that there are no substitutes for modeling to attain depths to magnetic sources. In this study, it was necessary to apply much filtering to the totalfield magnetic anomaly data in order to achieve correct depth estimates. However, filtering does not always separate the regional field and interference from nearby sources from the anomaly of interest very effectively, as was seen in the analytic signal amplitude over the buried volcanics. Also, filtering may remove part of the signal of interest and the true effects of filtering on the data are not always known. Modeling the regional field as well as nearby sources is required to fit the true data and adds a higher degree of certainty to the depth estimates. The automated interpretation techniques examined in this study should only be used to get a general idea of the magnetic contact locations and depths in large data sets. Potential-field modeling should be performed over individual anomalies of interest to honor the data and achieve the best depth estimates. 


\section{Suggested Improvements}

There remains a need for testing over additional targets, preferably over sources with several different body geometries, depths and magnetizations, to get a better feel for

the overall performance of these depth-to-source methods. In addition, the problems of remanent magnetization leading to offset contact locations when using the horizontal gradient method and a lack of a proper constraint for retaining correct depth solutions provided by the enhanced analytic signal method need to be addressed.

Applying a pseudo-gravity transformation to data in this study with parameters corresponding to the ambient geomagnetic field resulted in reasonable depth estimates over thin, shallow sources. However, it easy to see that if the magnetization vector has an orientation that is especially far from the input parameters for the pseudo-gravity transform, the shape of the magnitude of the horizontal gradient will not resemble that of the analytic signal over contacts, and poor or no depth estimates will result (Figure 45). In such cases, it is important to determine the magnetization vector before applying a pseudo-gravity transform. A good method for determining this vector, which requires comparing the analytic signal amplitude with the horizontal gradient magnitude of the pseudo-gravity over contacts, has been proposed by Roest and Pilkington (1993). 


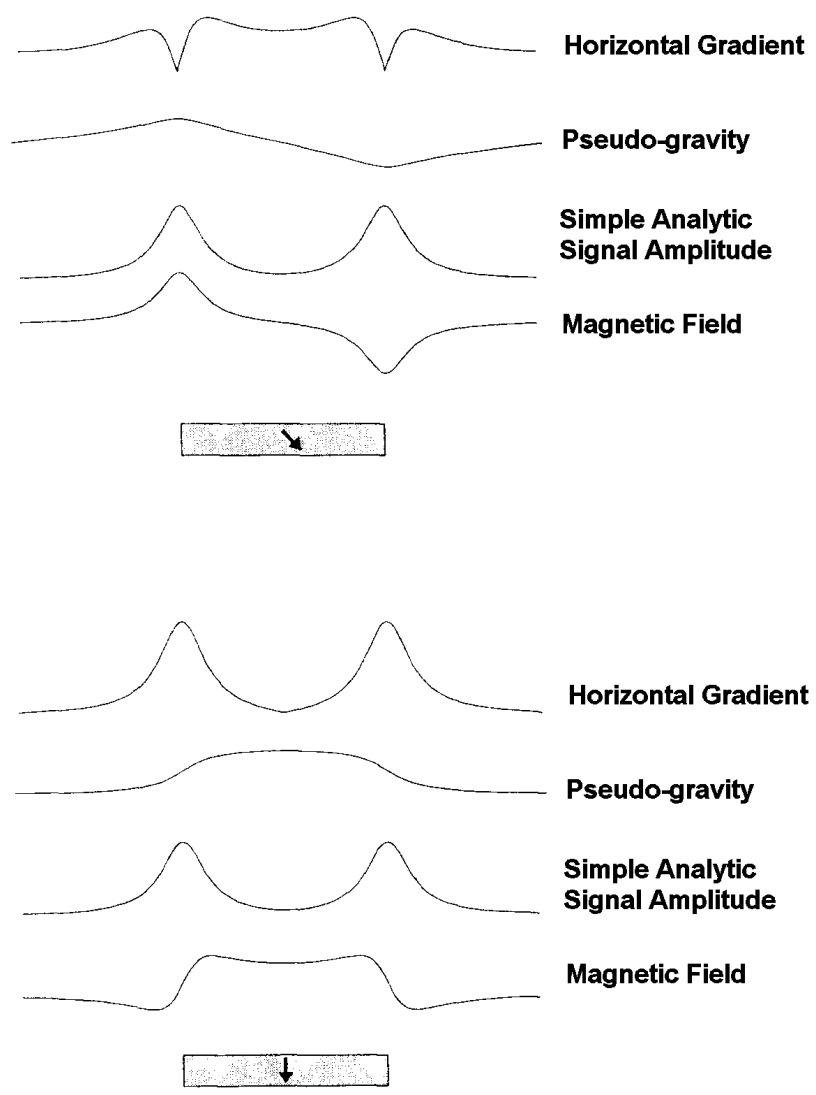

Figure 45. Shape of the two-dimensional horizontal gradient magnitude of the pseudo-gravity field over a contact when the source magnetization is not vertical (top). The source magnetization is vertical in the bottom image (adapted from Roest and Pilkington, 1993).

In this study, a depth-error threshold of $\leq 50 \%$ for the second order analytic signal magnitude grid was used instead of $\leq 15 \%$ to acquire a significant number of solutions. For the enhanced analytic signal method, there is a need for an algorithm that finds peaks in the higher-order analytic signal amplitudes using functions that properly describe their theoretical shapes over magnetic contacts. This would allow for the calculated error to be indicative of the reliability of the depth solutions, and many erroneous depths would be eliminated from the output. 
APPENDIX A: Depth-to-source maps over the Los Reales Landfill

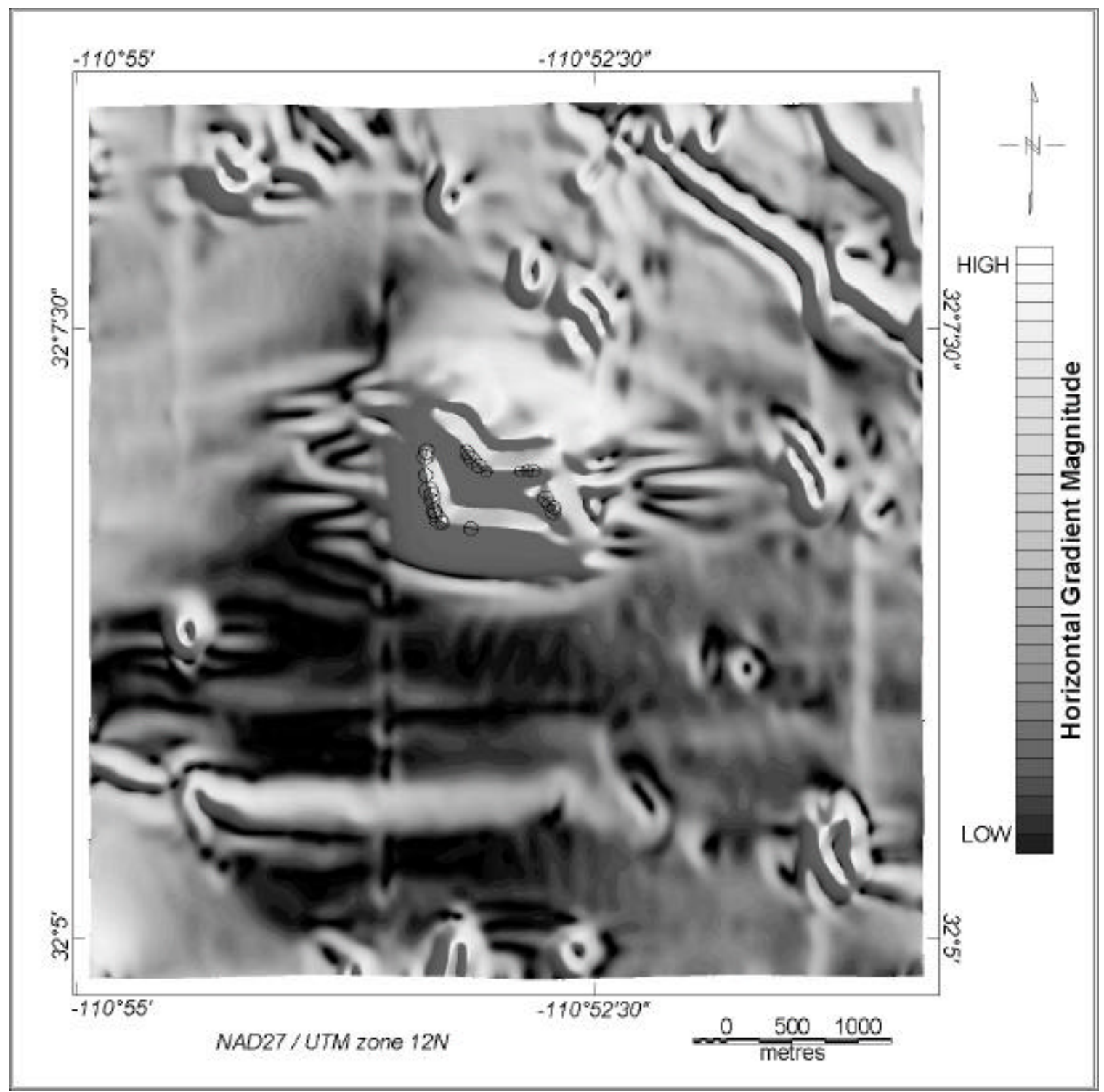

A1. Depth to source map over the Los Reales Landfill using the horizontal gradient method. The circles are centered over the contacts and their sizes are relative to the depth from the airplane. 


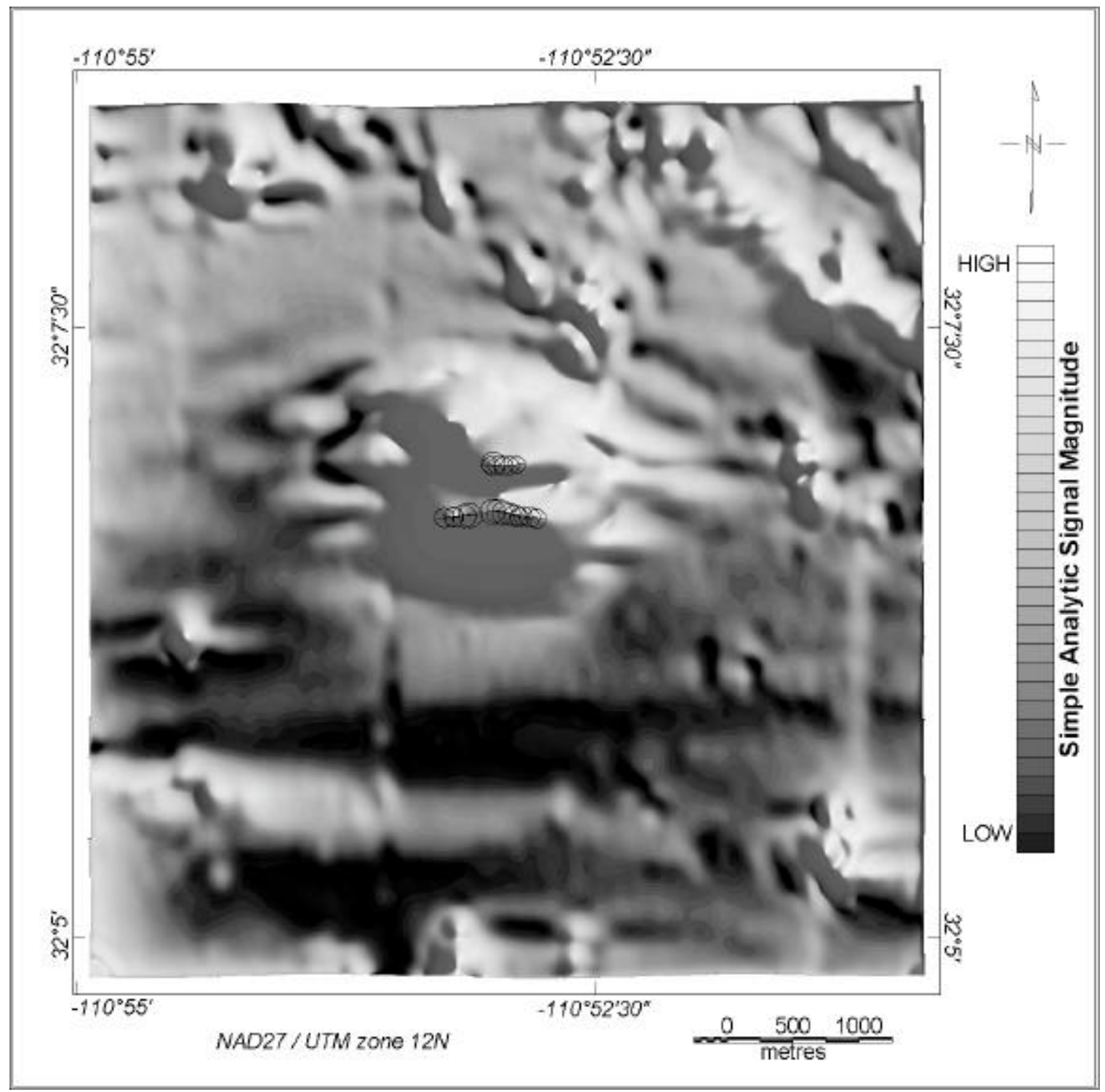

A2. Depth to source map over the Los Reales Landfill using the simple analytic signal method. 


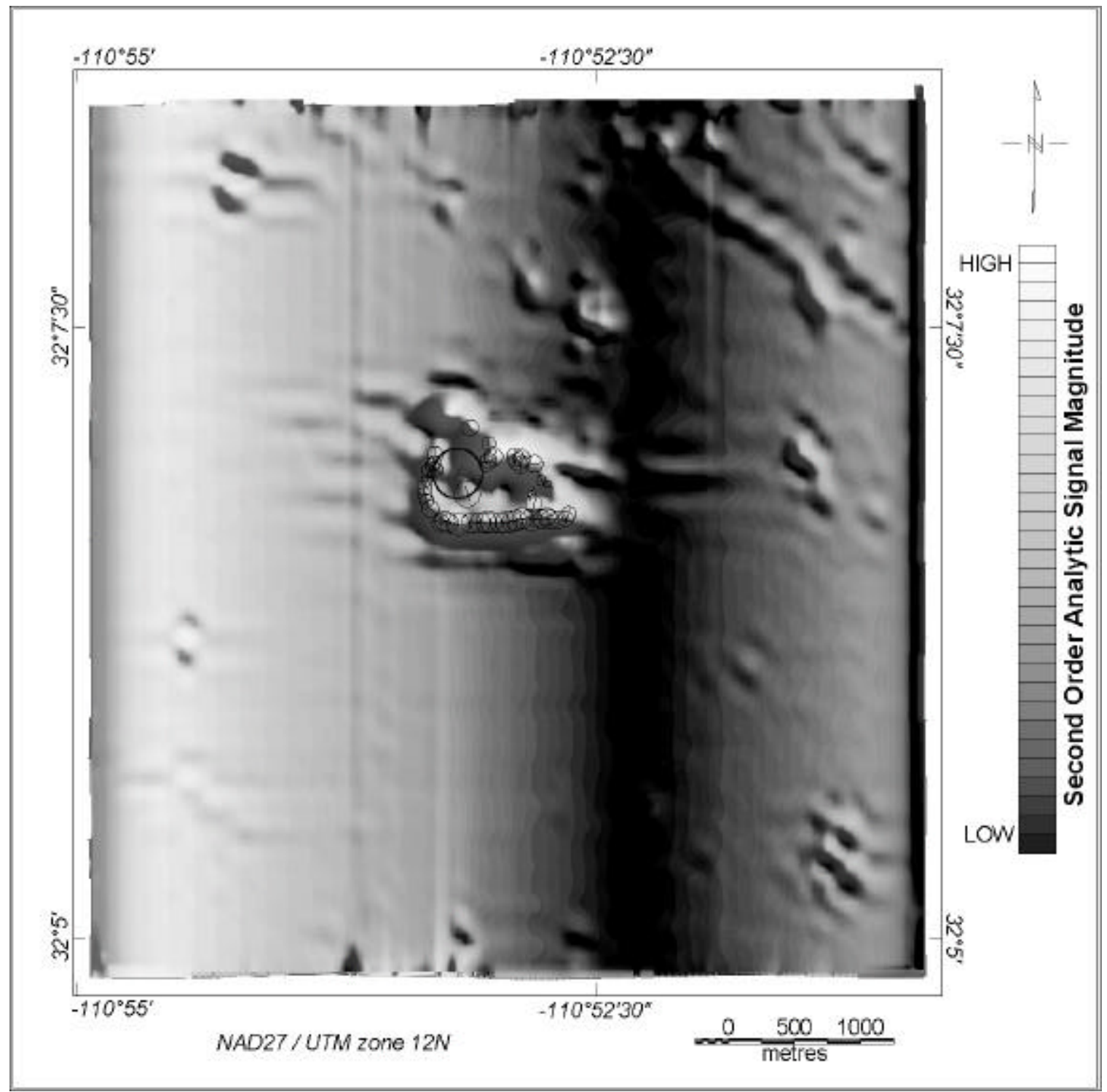

A3. Depth to source map over the Los Reales Landfill using the enhanced analytic signal method (step model). 


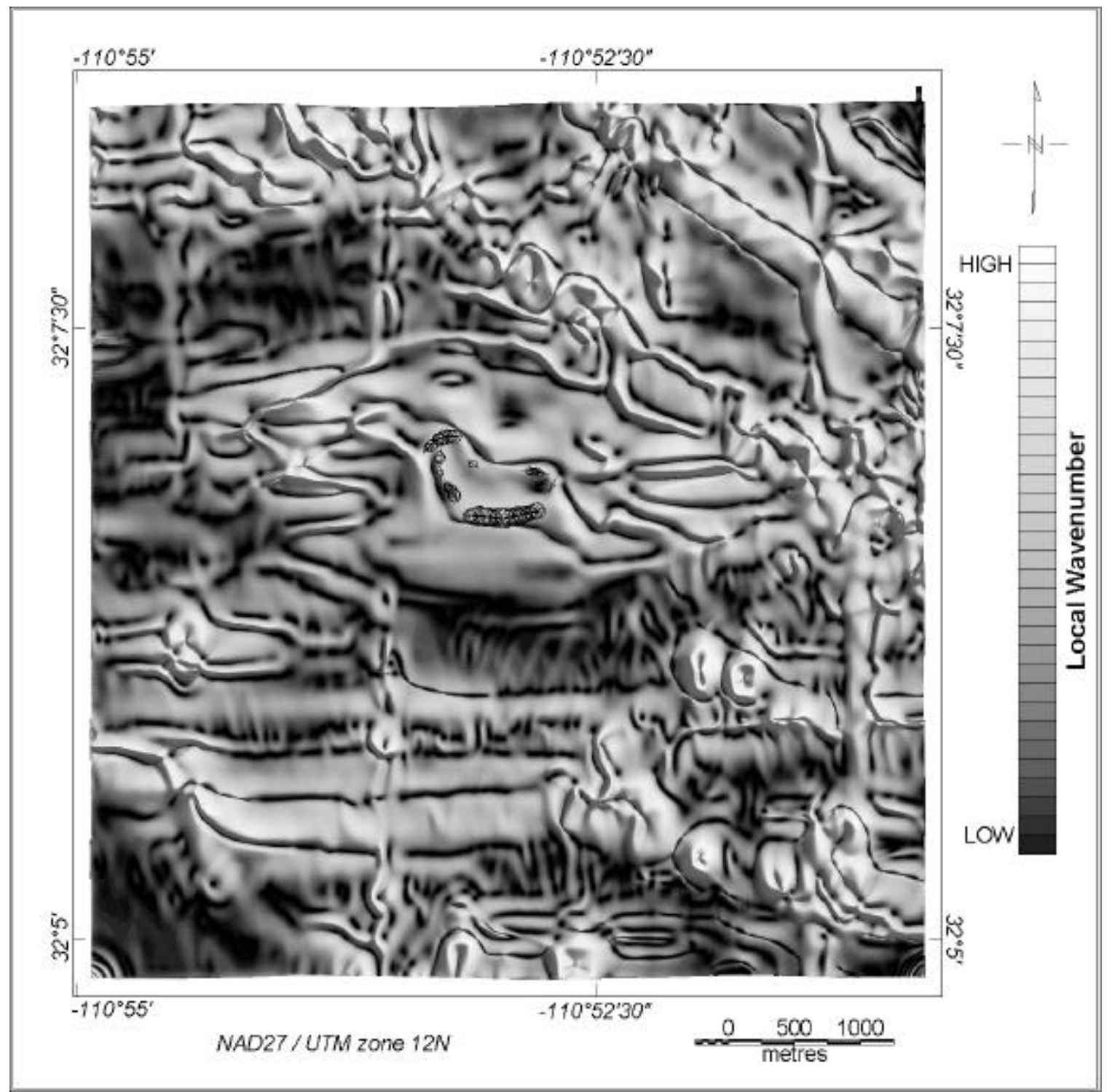

A4. Depth to source map over the Los Reales Landfill using the local wavenumber method. 


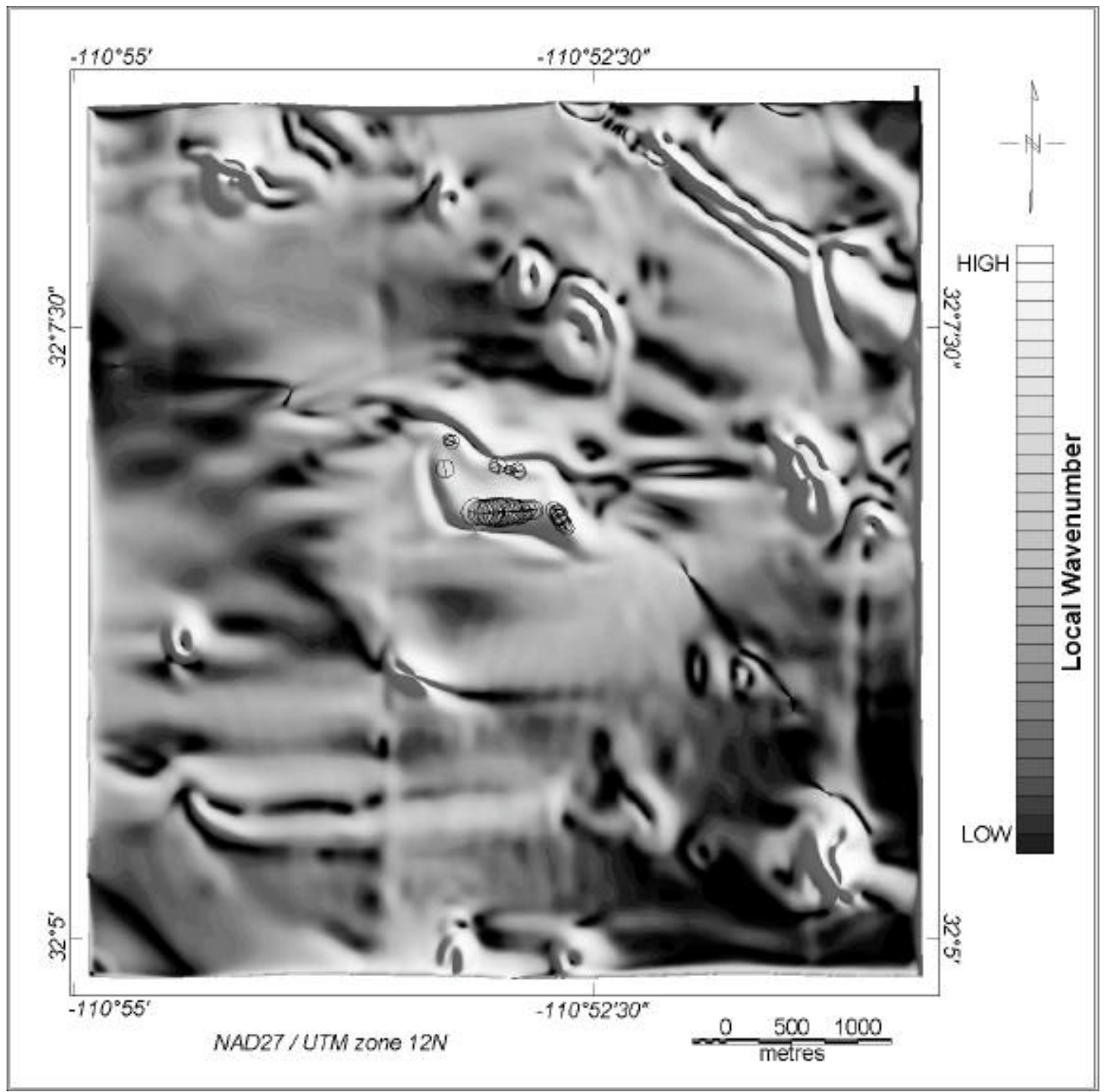

A5. Depth to source map over the Los Reales Landfill using the local wavenumber method applied to the vertical integral of the total magnetic field. 


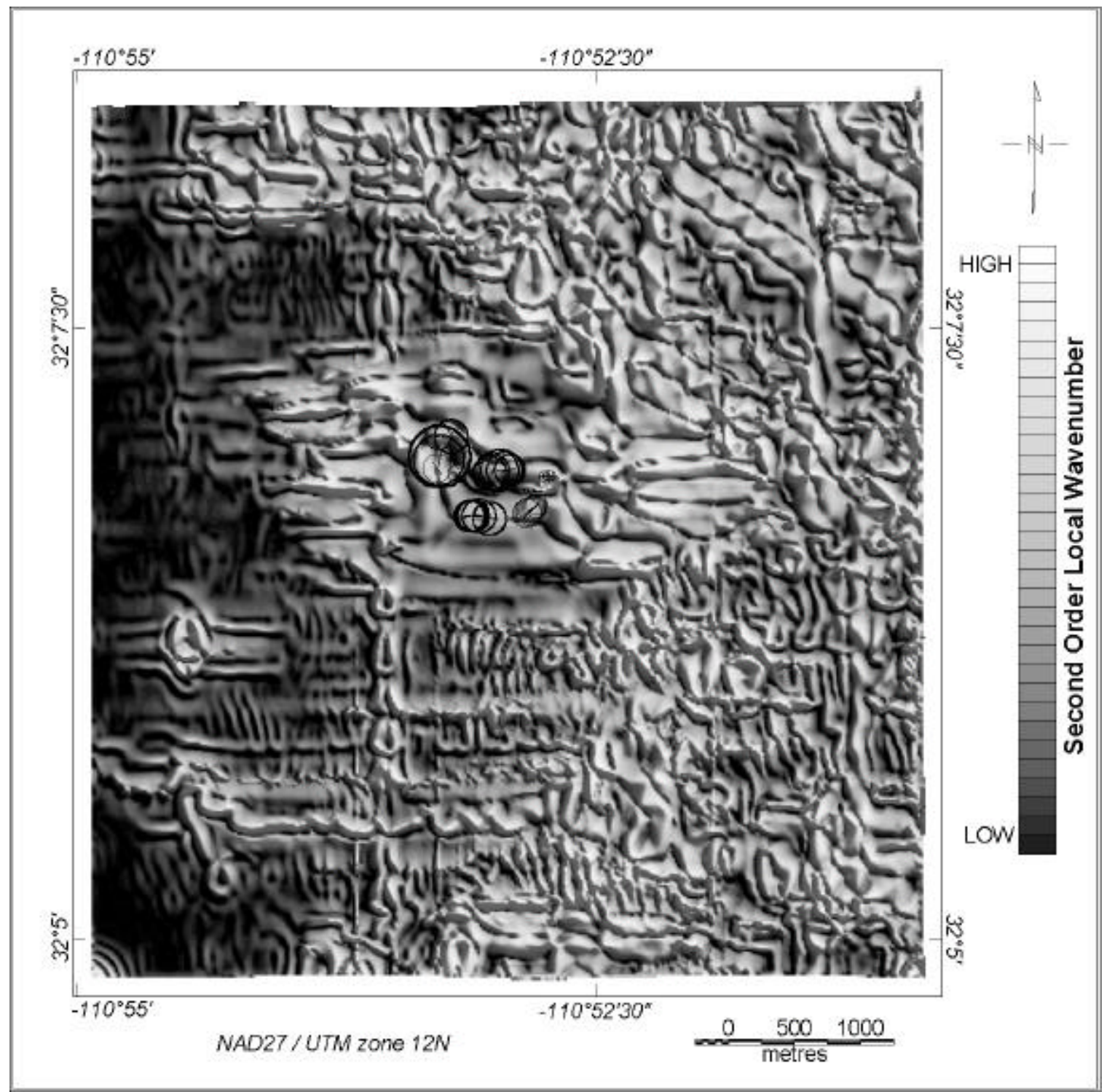

A6. Depth to source map over the Los Reales Landfill using the enhanced local wavenumber method. 


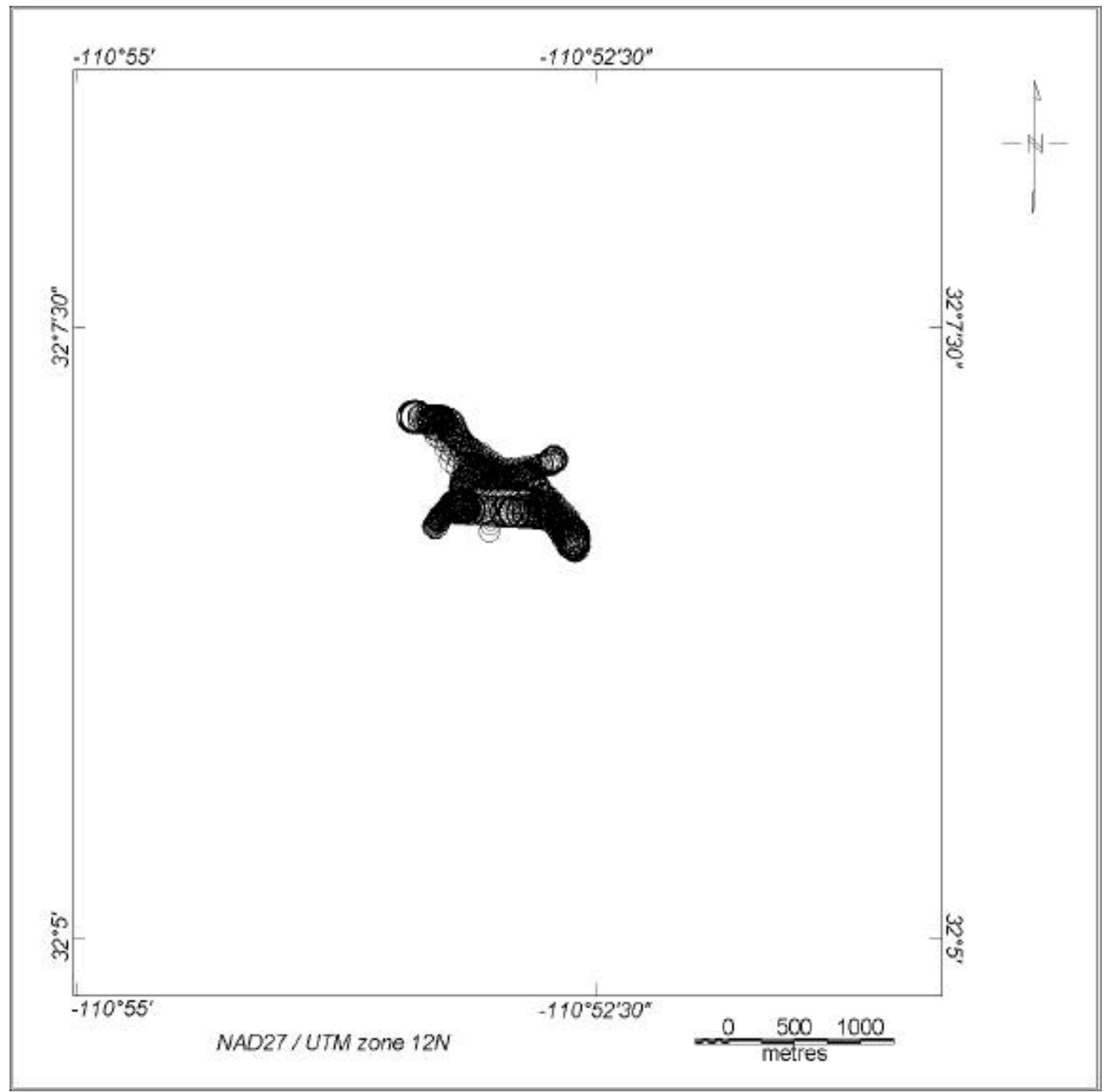

A7. Depth to source map over the Los Reales Landfill using Euler deconvolution. 


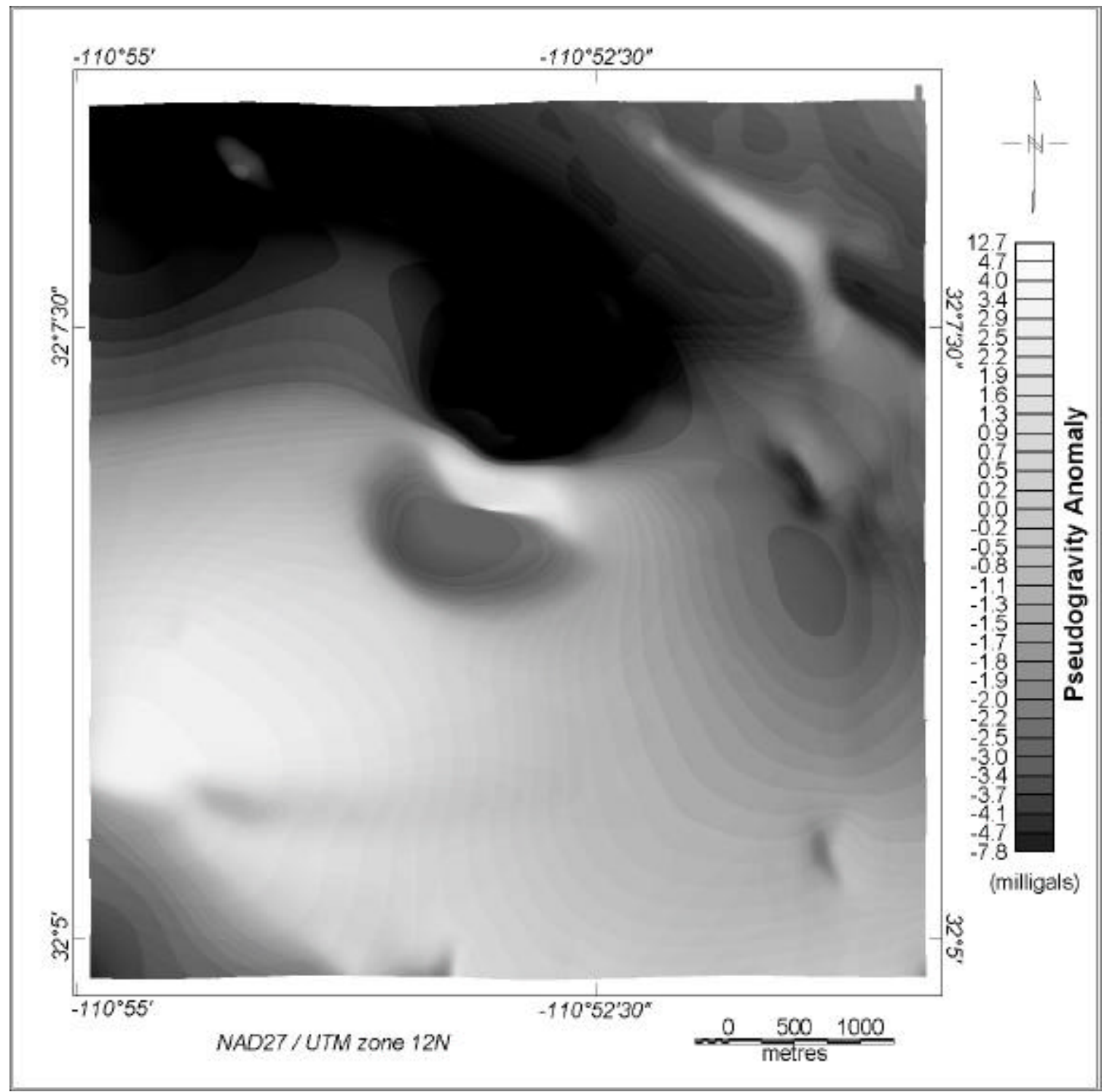

A8. Pseudo-gravity anomaly map over the Los Reales Landfill. 


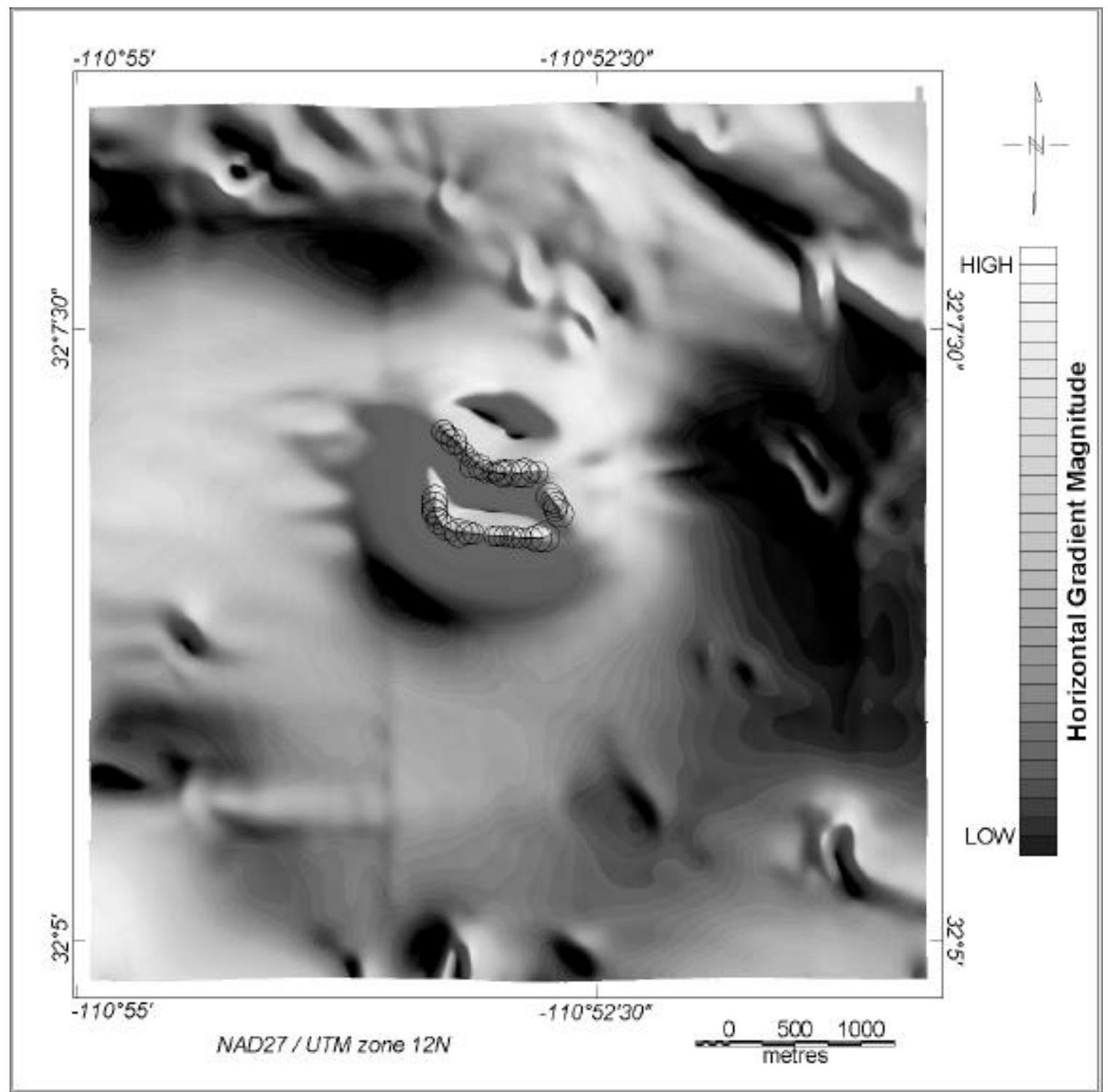

A9. Depth to source map over the Los Reales Landfill using the horizontal gradient method applied to the pseudo-gravity field. 


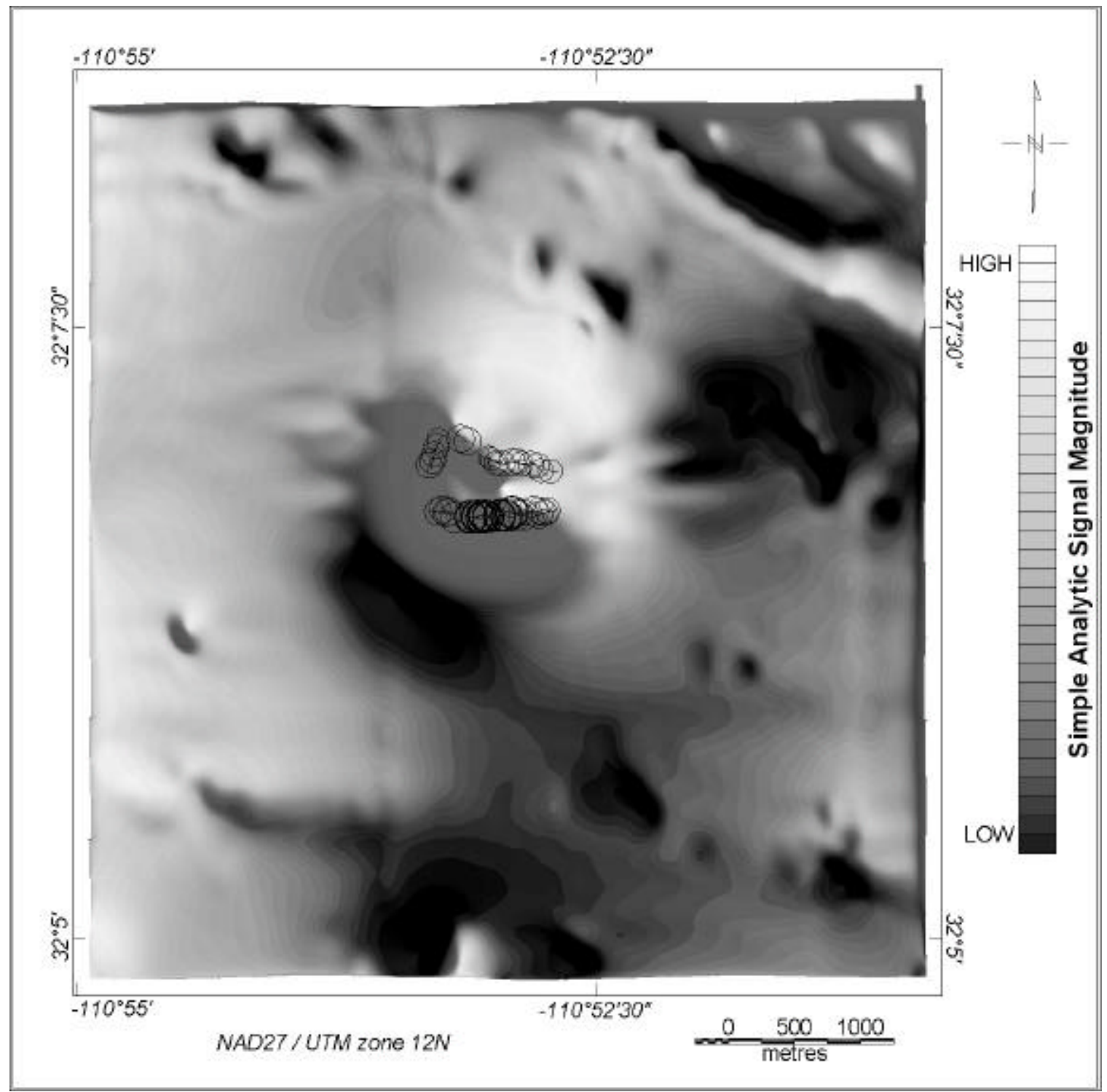

A10. Depth to source map over the Los Reales Landfill using the simple analytic signal method applied to the pseudo-gravity field. 


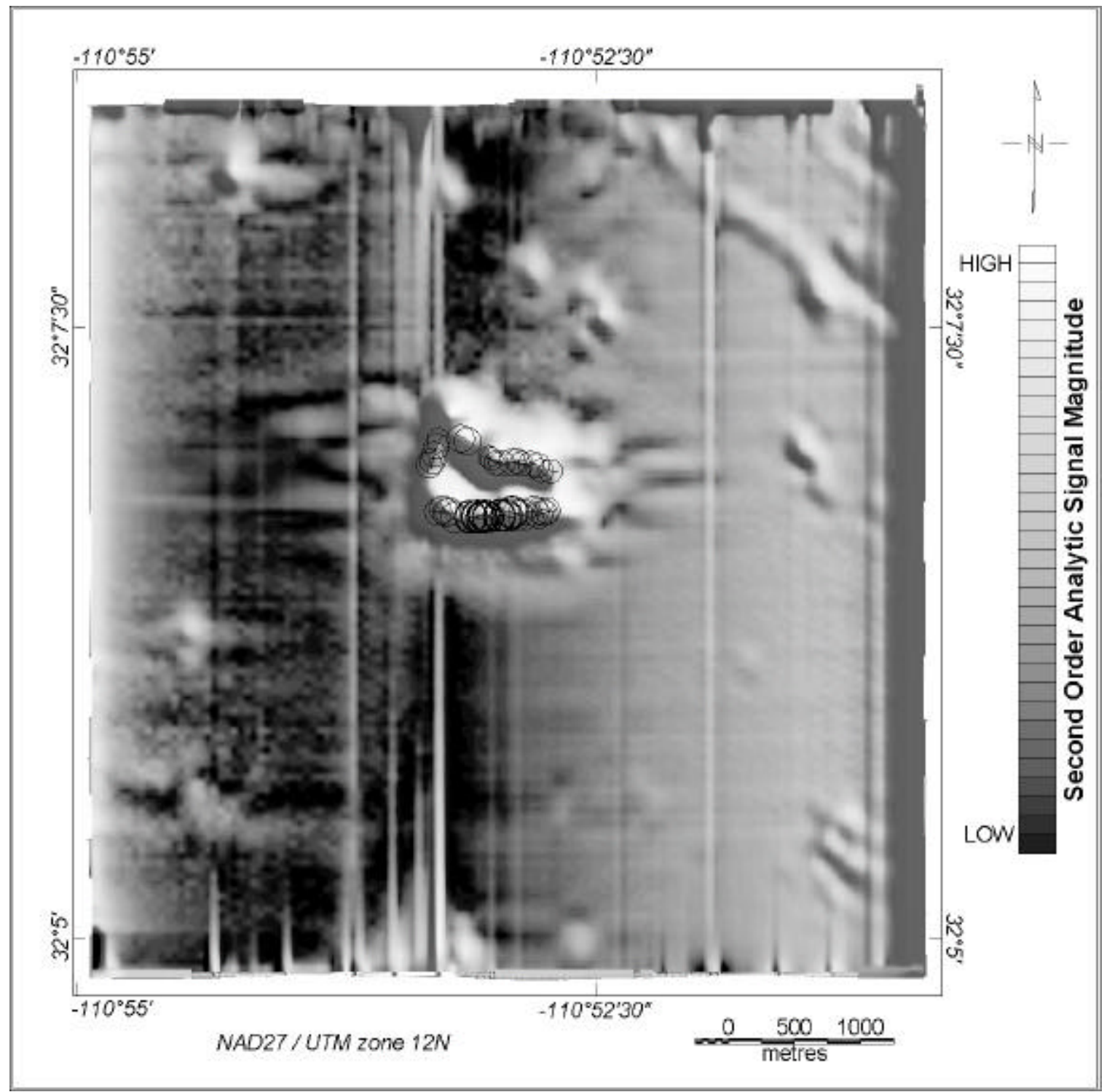

A11. Depth to source map over the Los Reales Landfill using the enhanced analytic signal method (step model) applied to the pseudo-gravity field. 


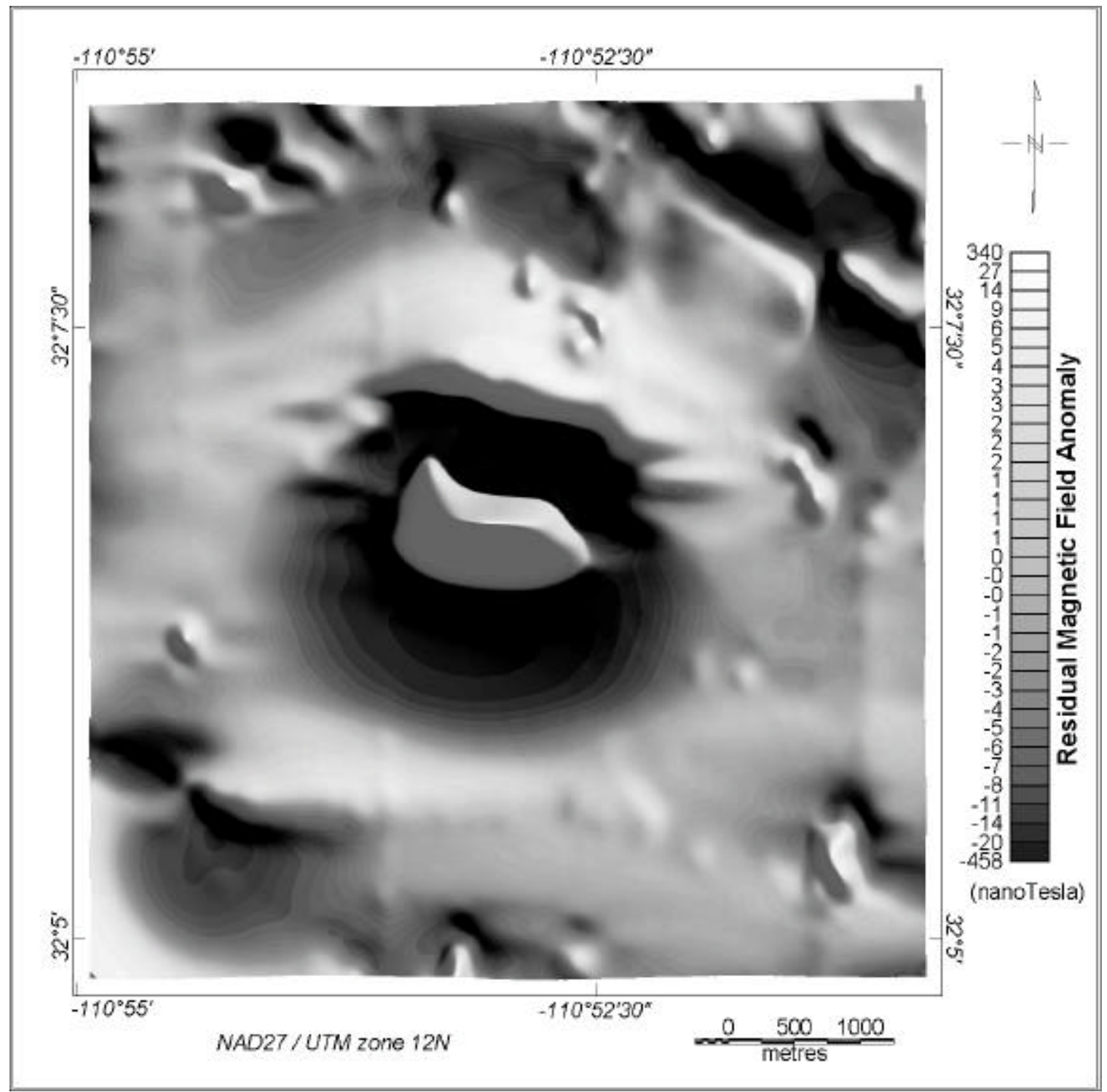

A12. High-pass filtered magnetic field anomaly map over the Los Reales Landfill. 


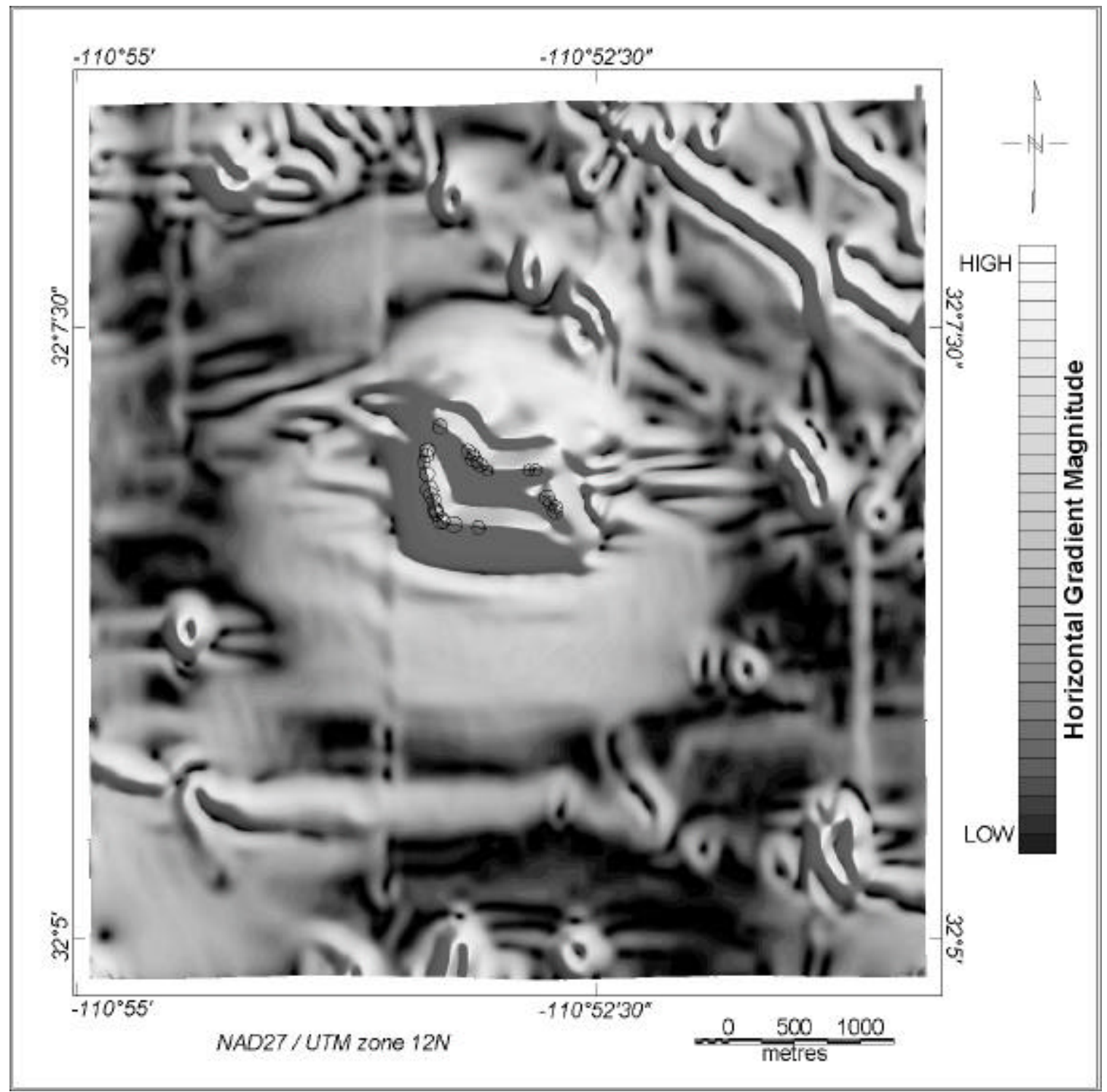

A13. Depth to source map over the Los Reales Landfill using the horizontal gradient method applied to the high-pass filtered data. 


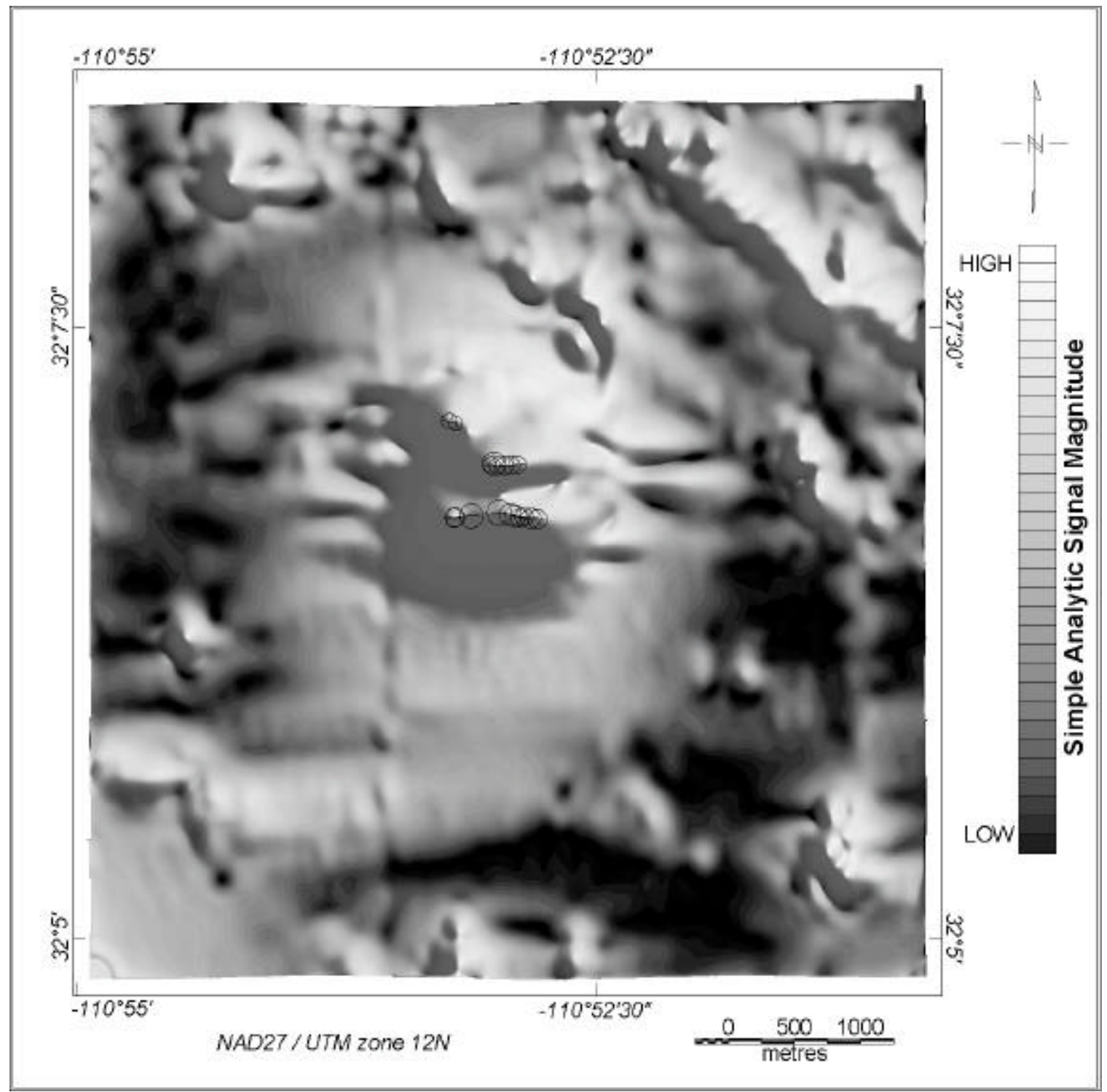

A14. Depth to source map over the Los Reales Landfill using the simple analytic signal method applied to the high-pass filtered data. 


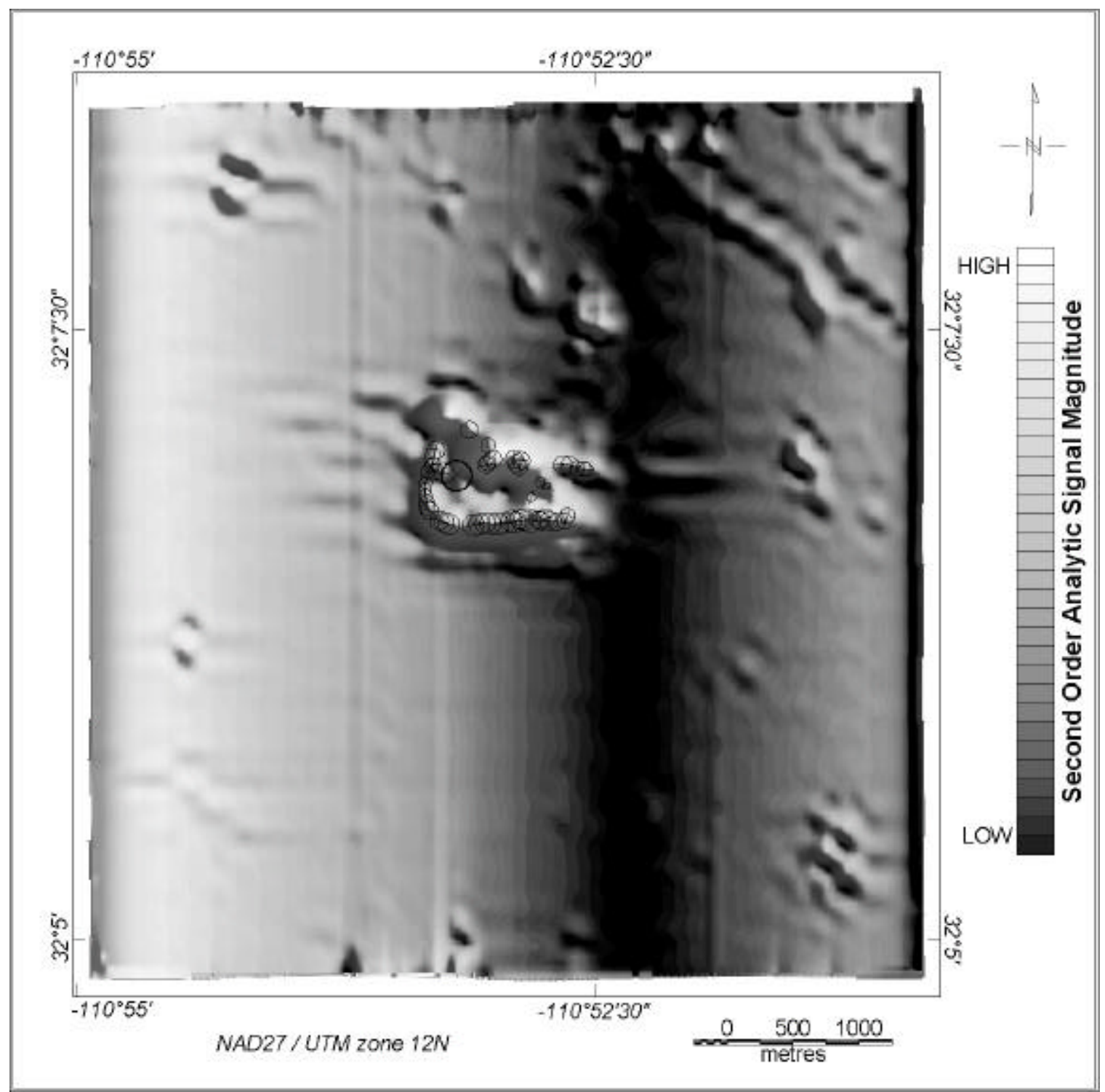

A15. Depth to source map over the Los Reales Landfill using the enhanced analytic signal method (step model) applied to the high-pass filtered data. 


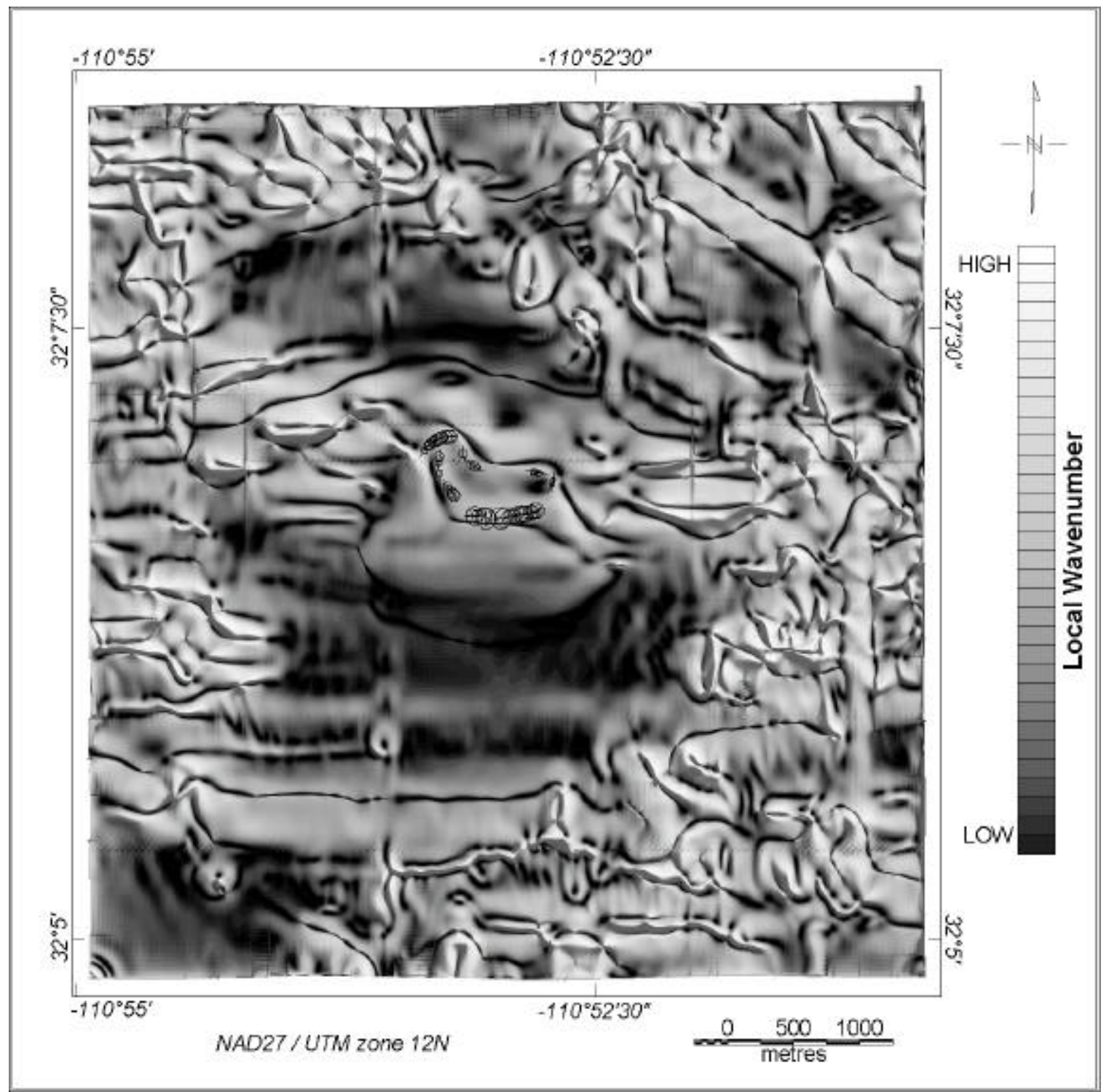

A16. Depth to source map over the Los Reales Landfill using the local wavenumber method applied to the high-pass filtered data. 


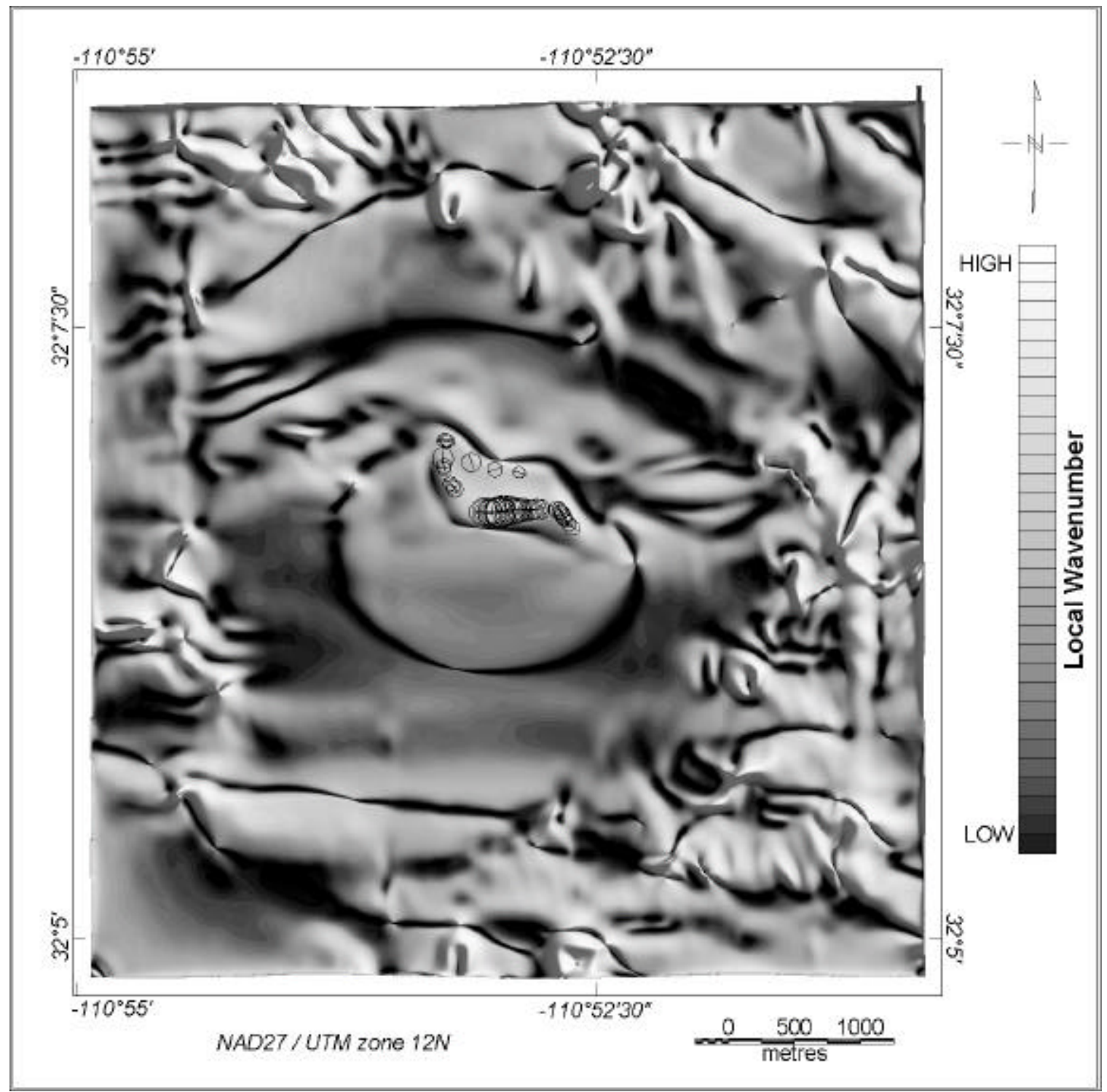

A17. Depth to source map over the Los Reales Landfill using the local wavenumber method applied to the vertical integral of the residual magnetic field. 


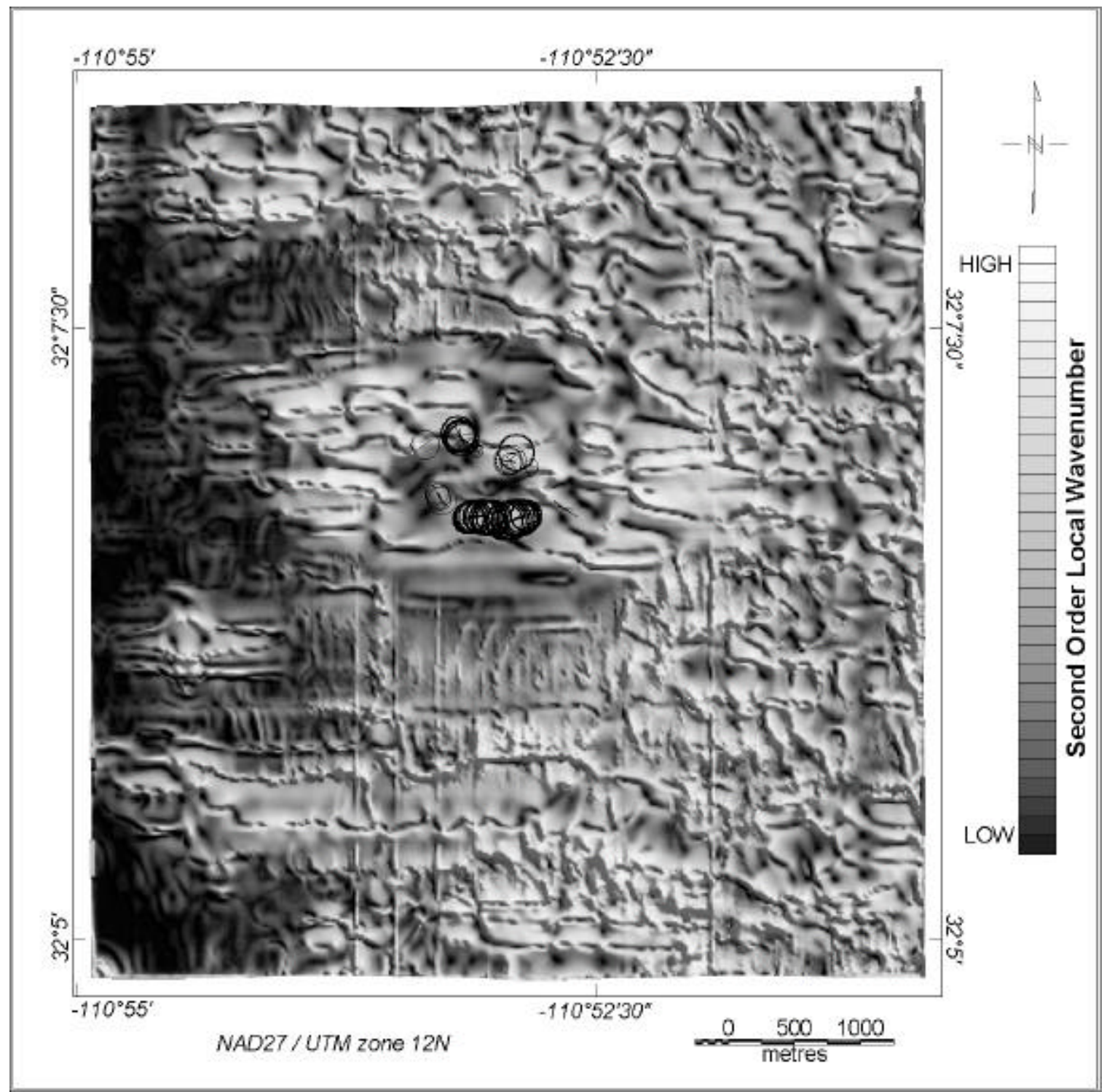

A18. Depth to source map over the Los Reales Landfill using the enhanced local wavenumber method applied to the high-pass filtered data. 


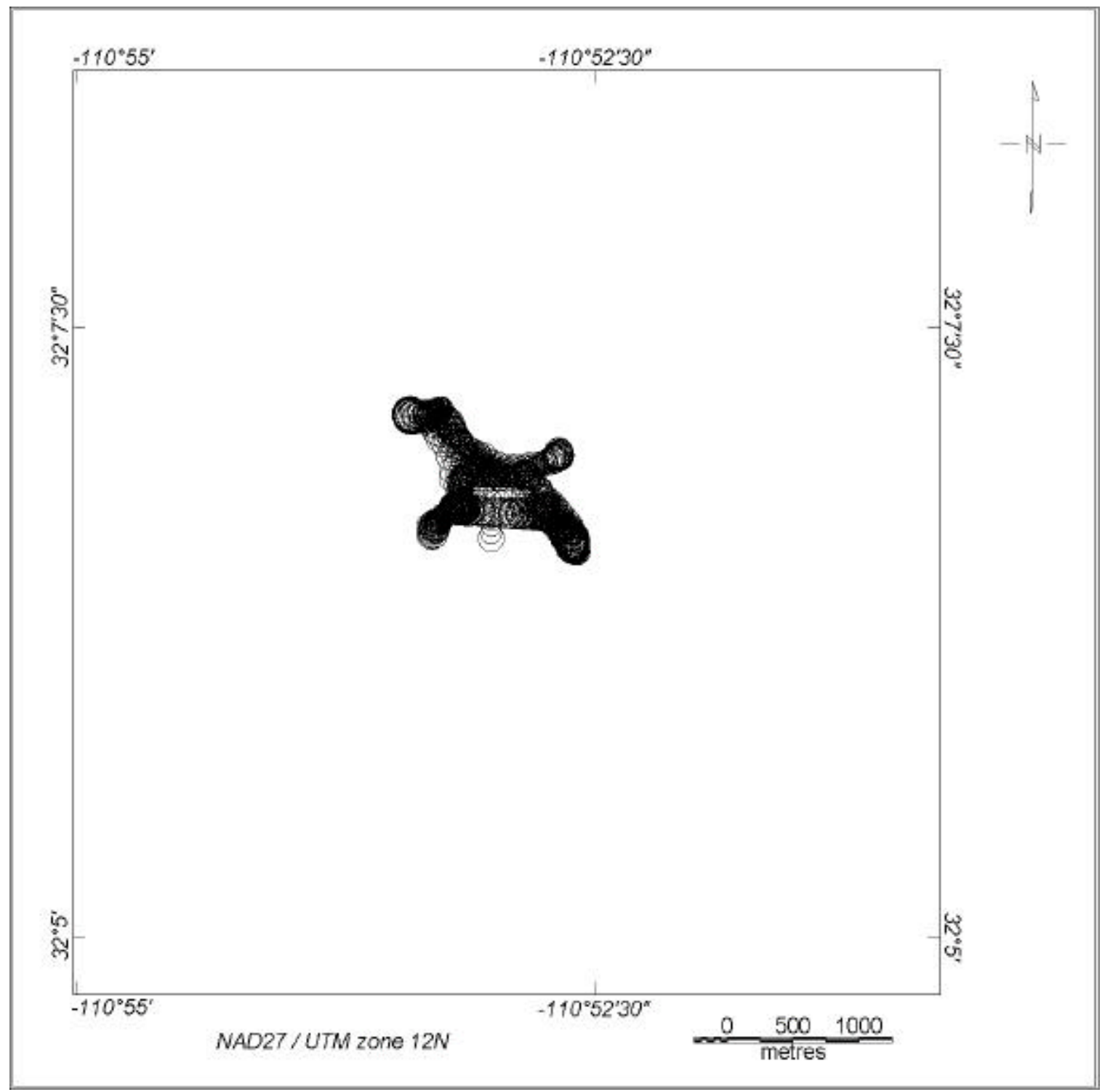

A19. Depth to source map over the Los Reales Landfill using Euler deconvolution applied to the high-pass filtered data. 


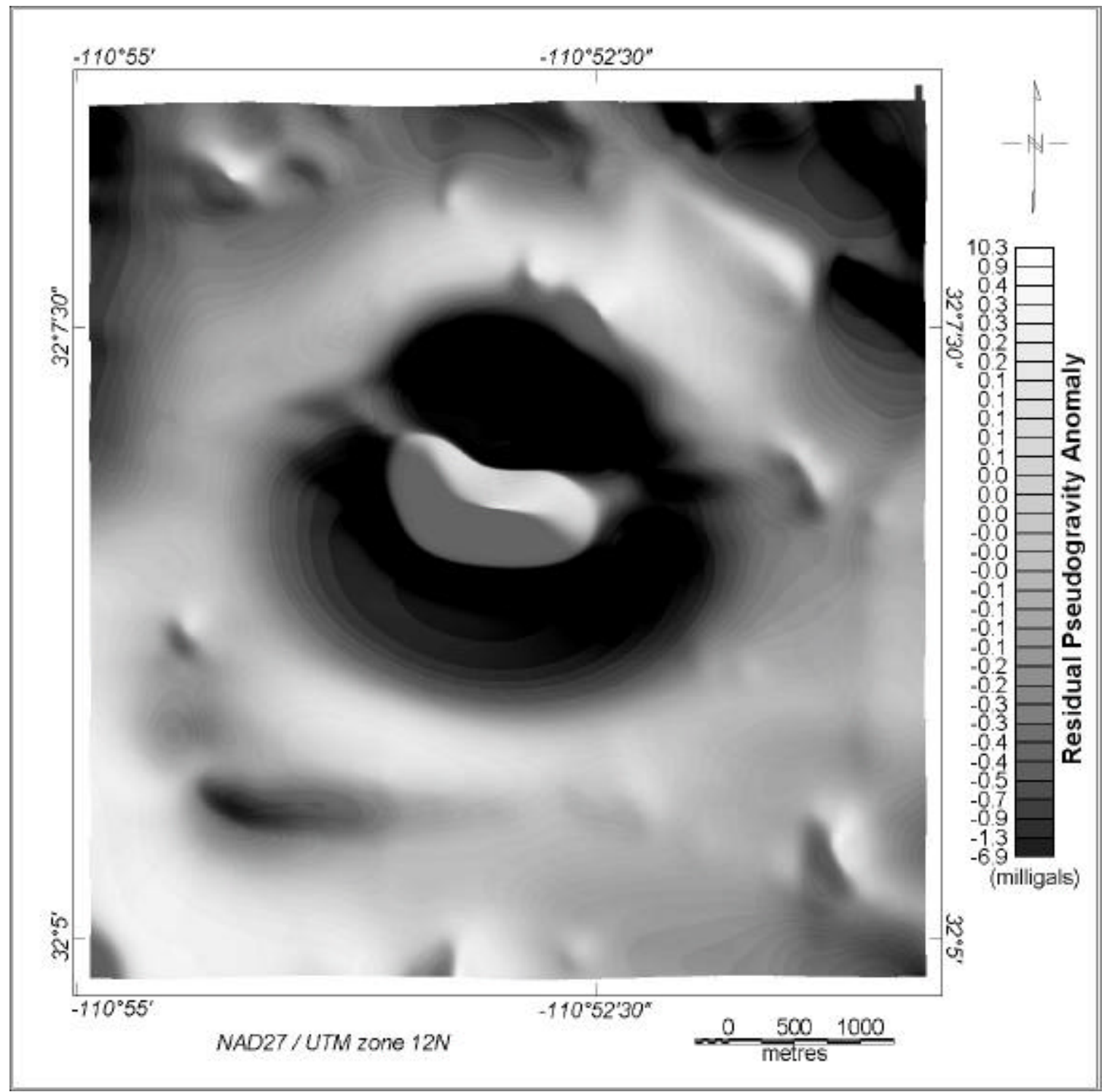

A20. High-pass filtered pseudo-gravity anomaly map over the Los Reales Landfill. 


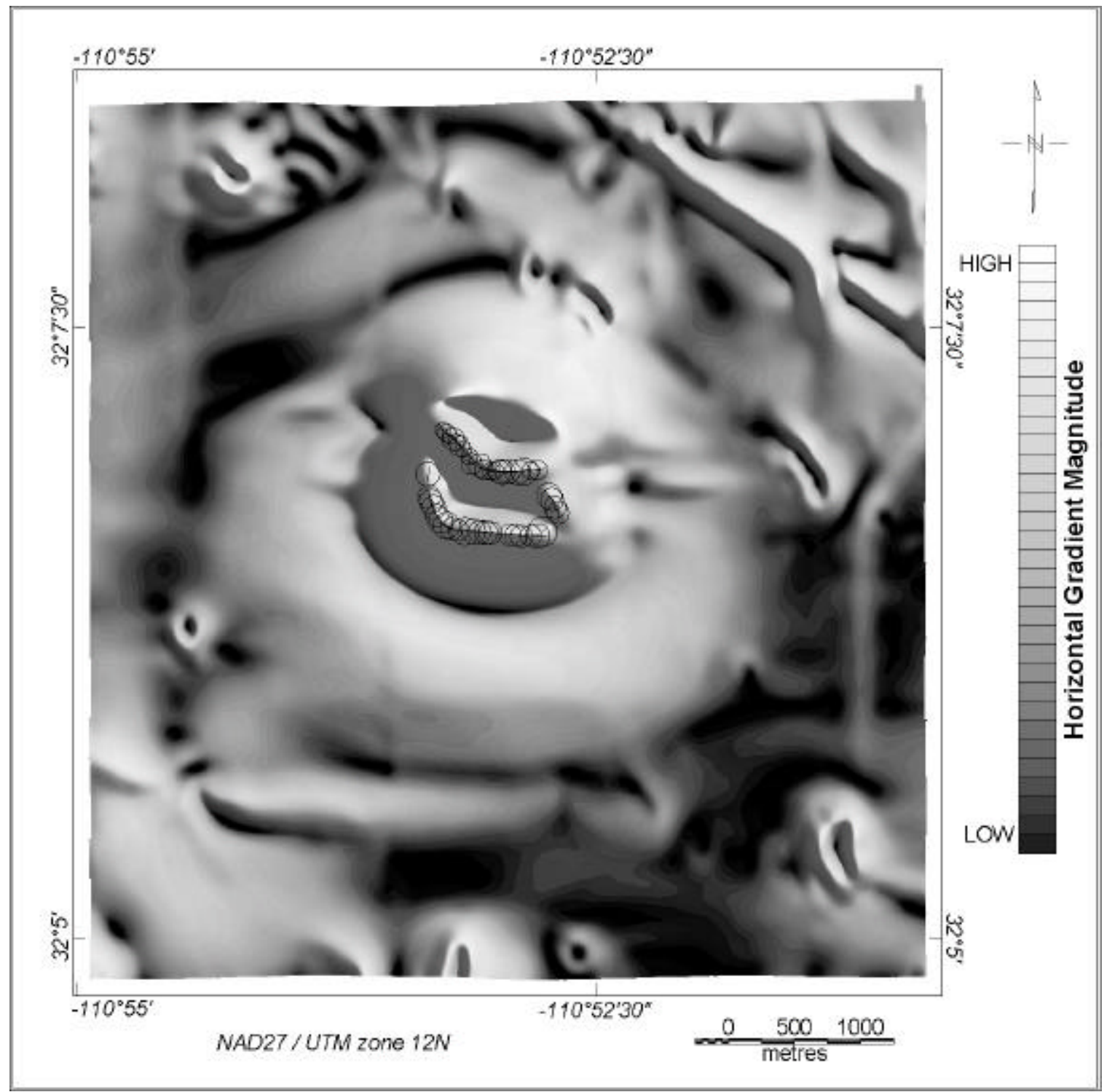

A21. Depth to source map over the Los Reales Landfill using the horizontal gradient method applied to the high-pass filtered pseudo-gravity field. 


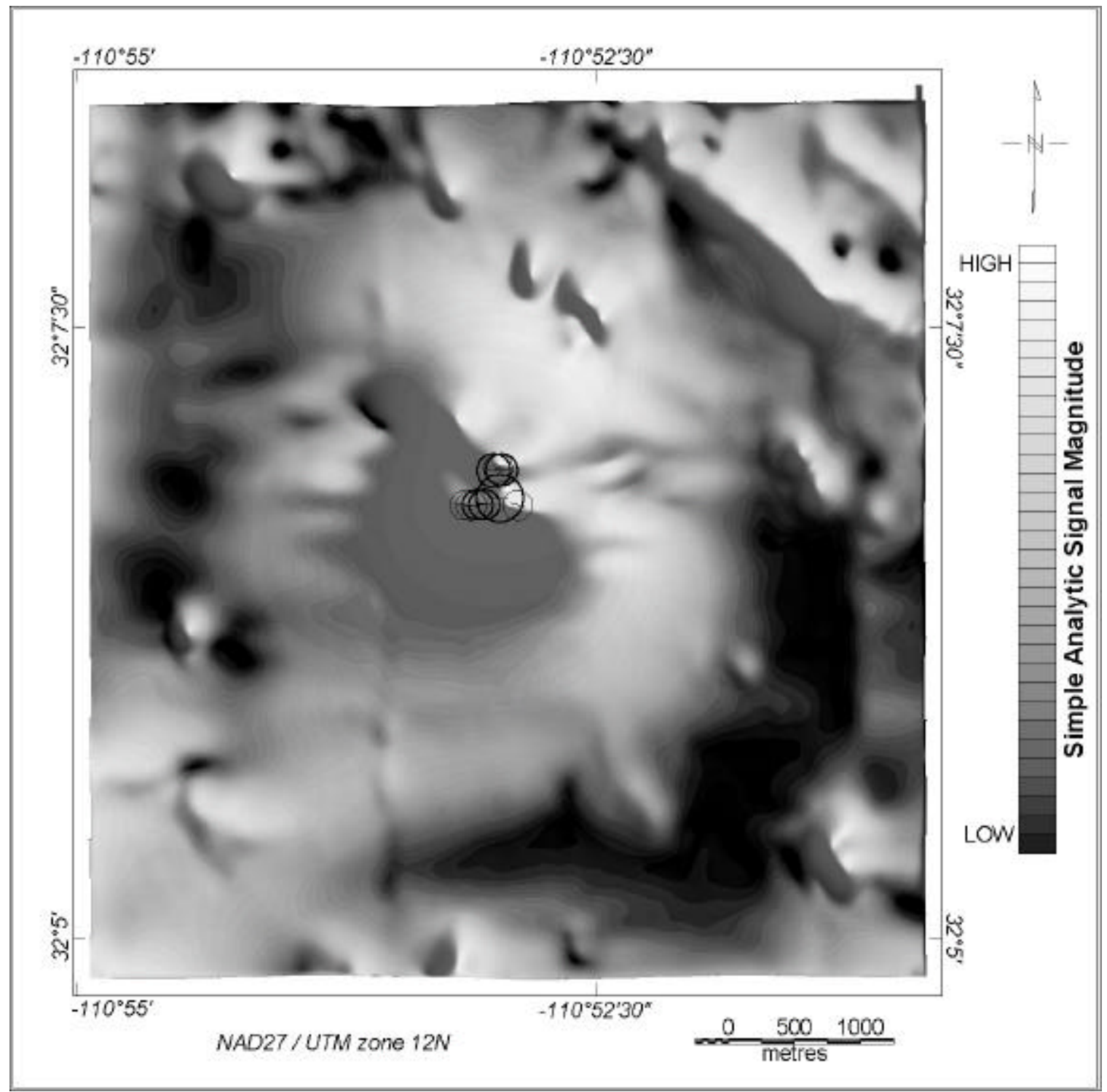

A22. Depth to source map over the Los Reales Landfill using the simple analytic signal method applied to the high-pass filtered pseudo-gravity field. 


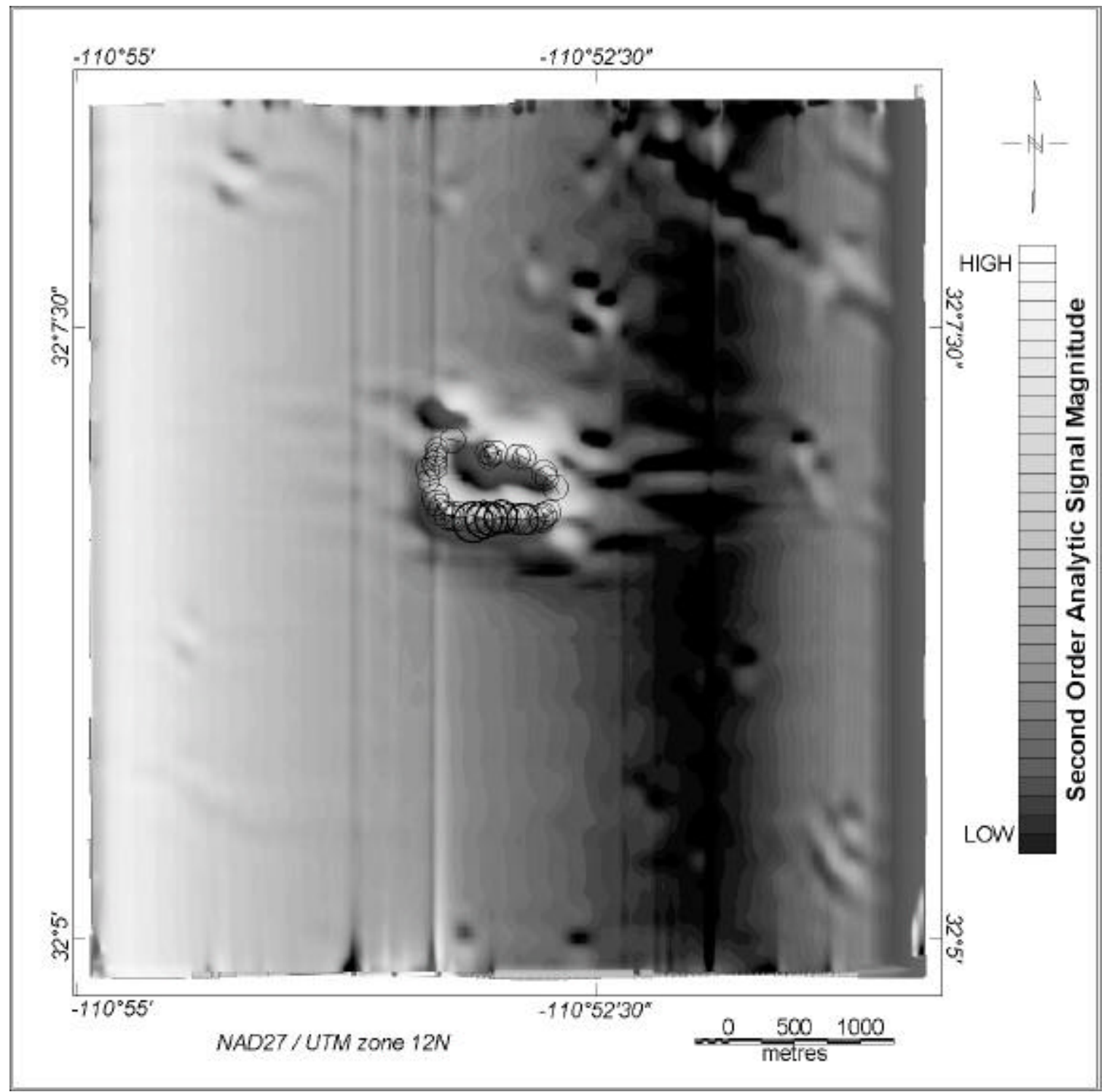

A23. Depth to source map over the Los Reales Landfill using the enhanced analytic signal method (step model) applied to the high-pass filtered pseudo-gravity field. 
APPENDIX B: Depth-to-source maps over Black Mountain

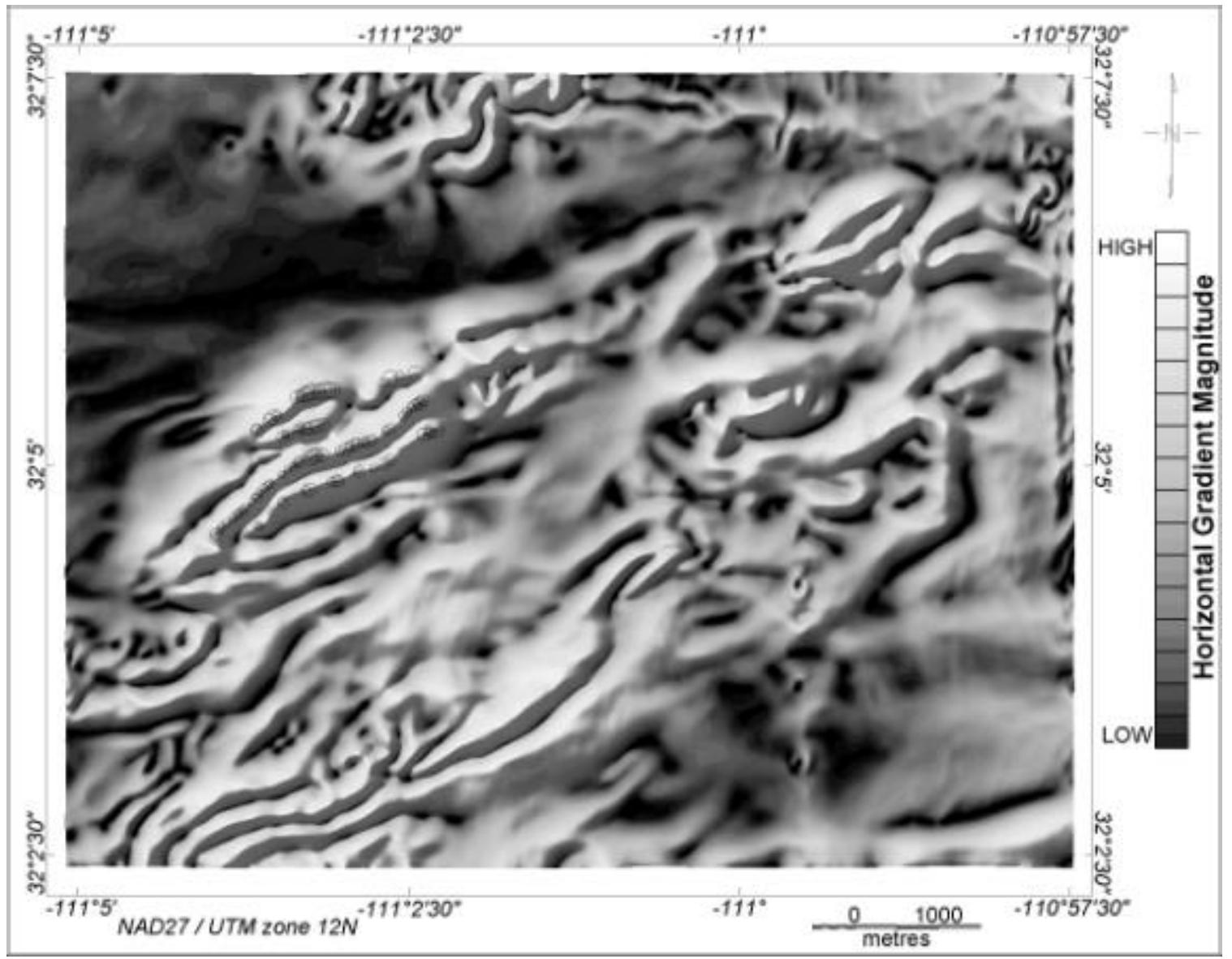

B1. Depth to source map over Black Mountain using the horizontal gradient method. The circles are centered over the contacts and their sizes are relative to the depth from the airplane. 


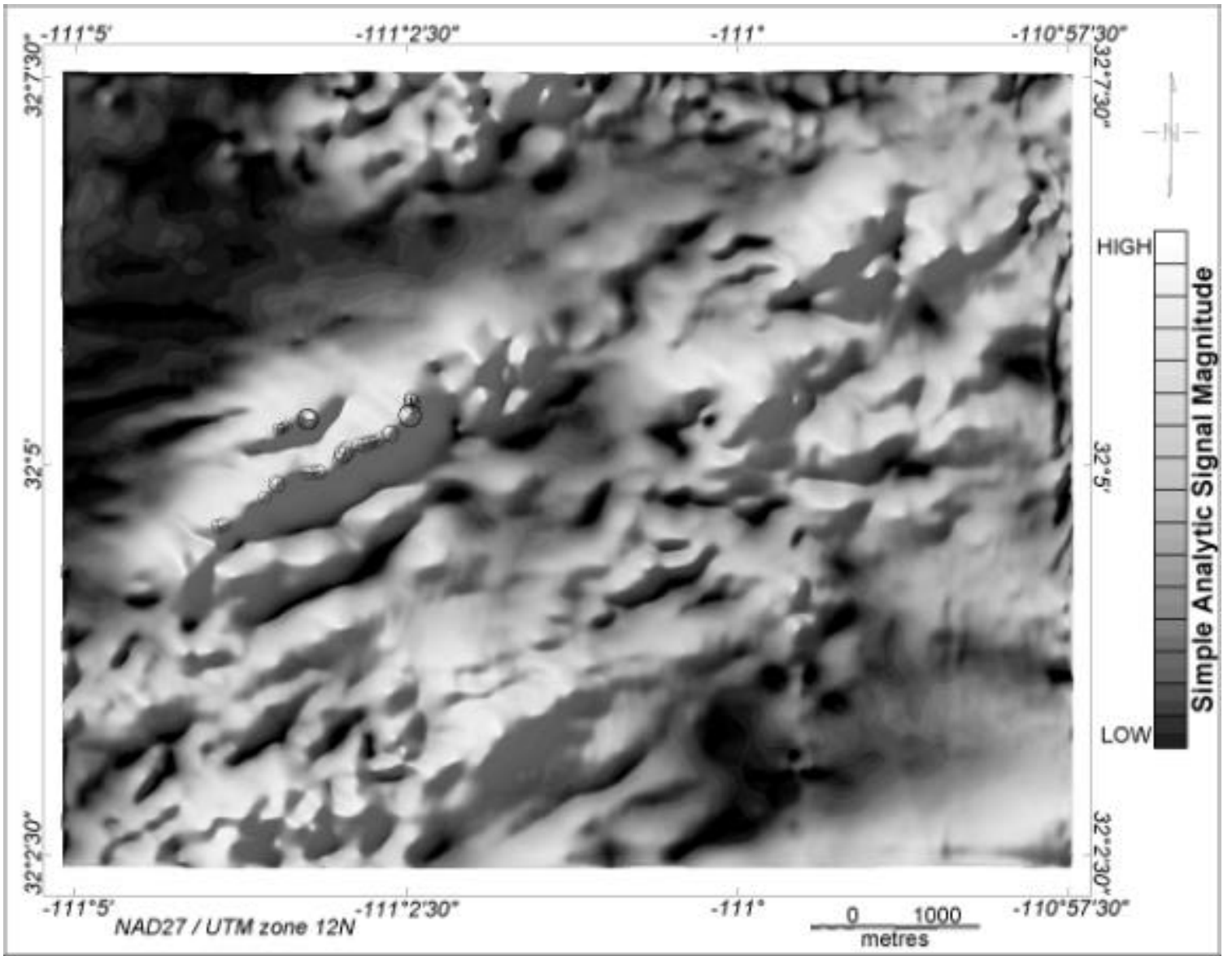

B2. Depth to source map over Black Mountain using the simple analytic signal method. 


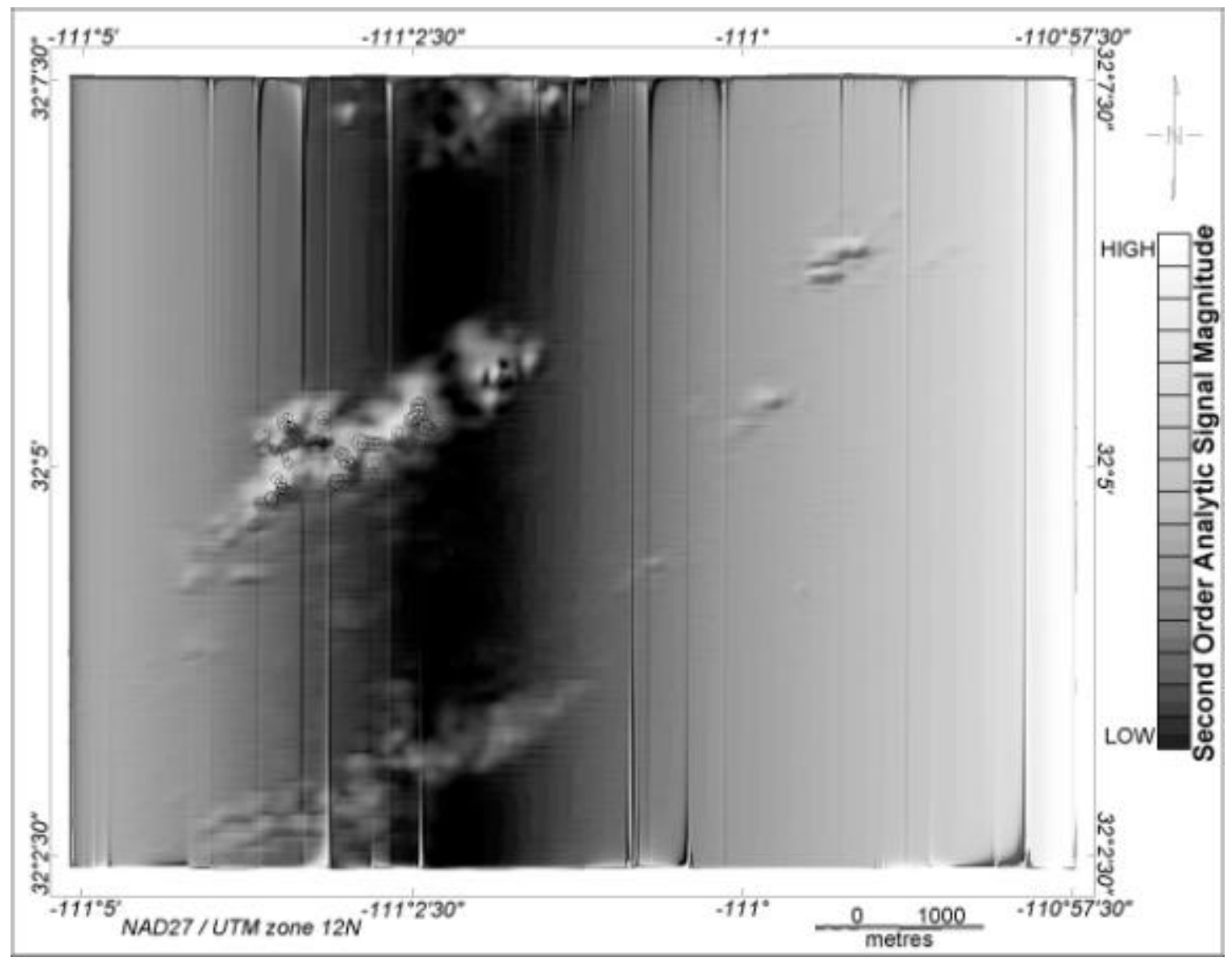

B3. Depth to source map over Black Mountain using the enhanced analytic signal method (step model). 


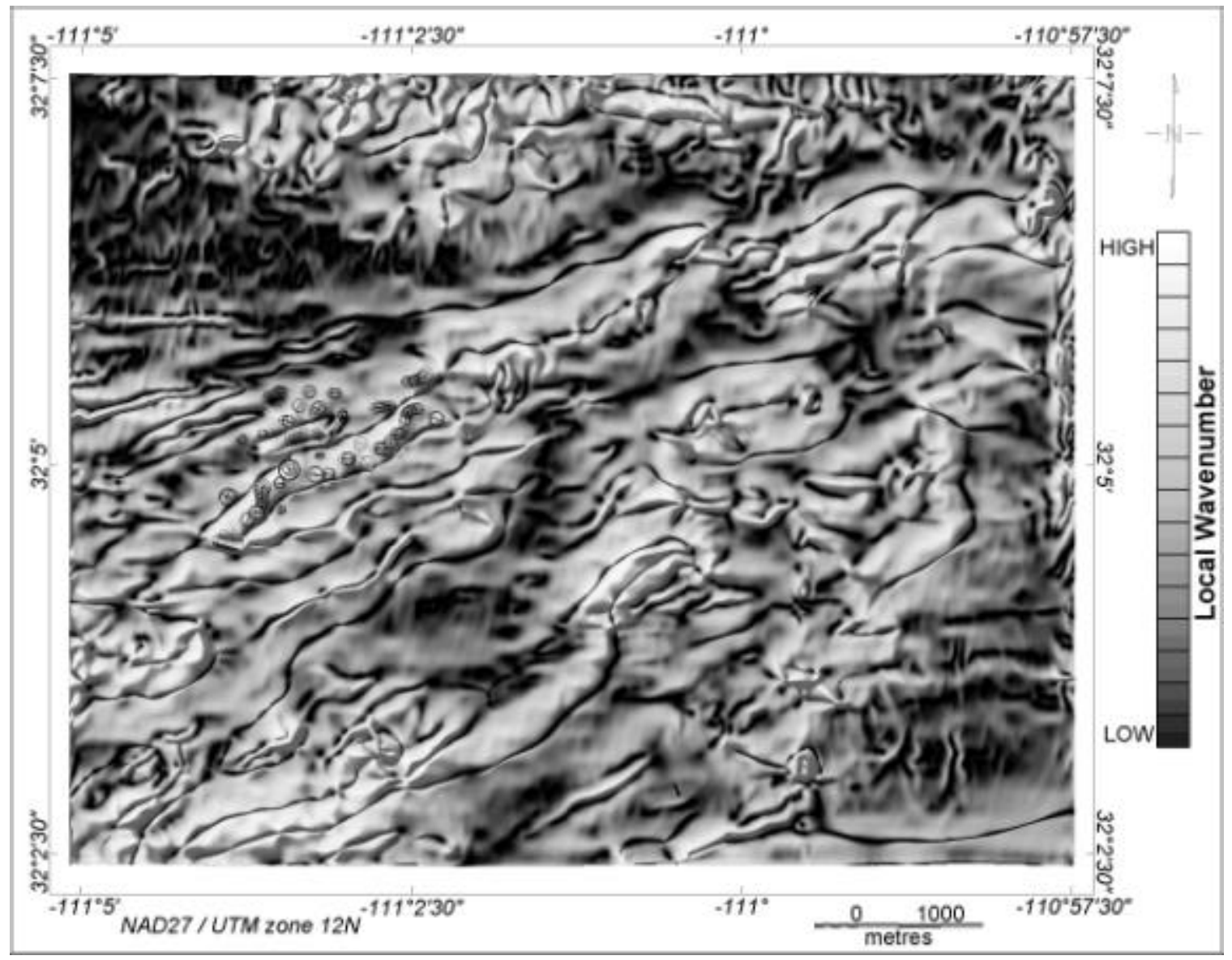

B4. Depth to source map over Black Mountain using the local wavenumber method. 


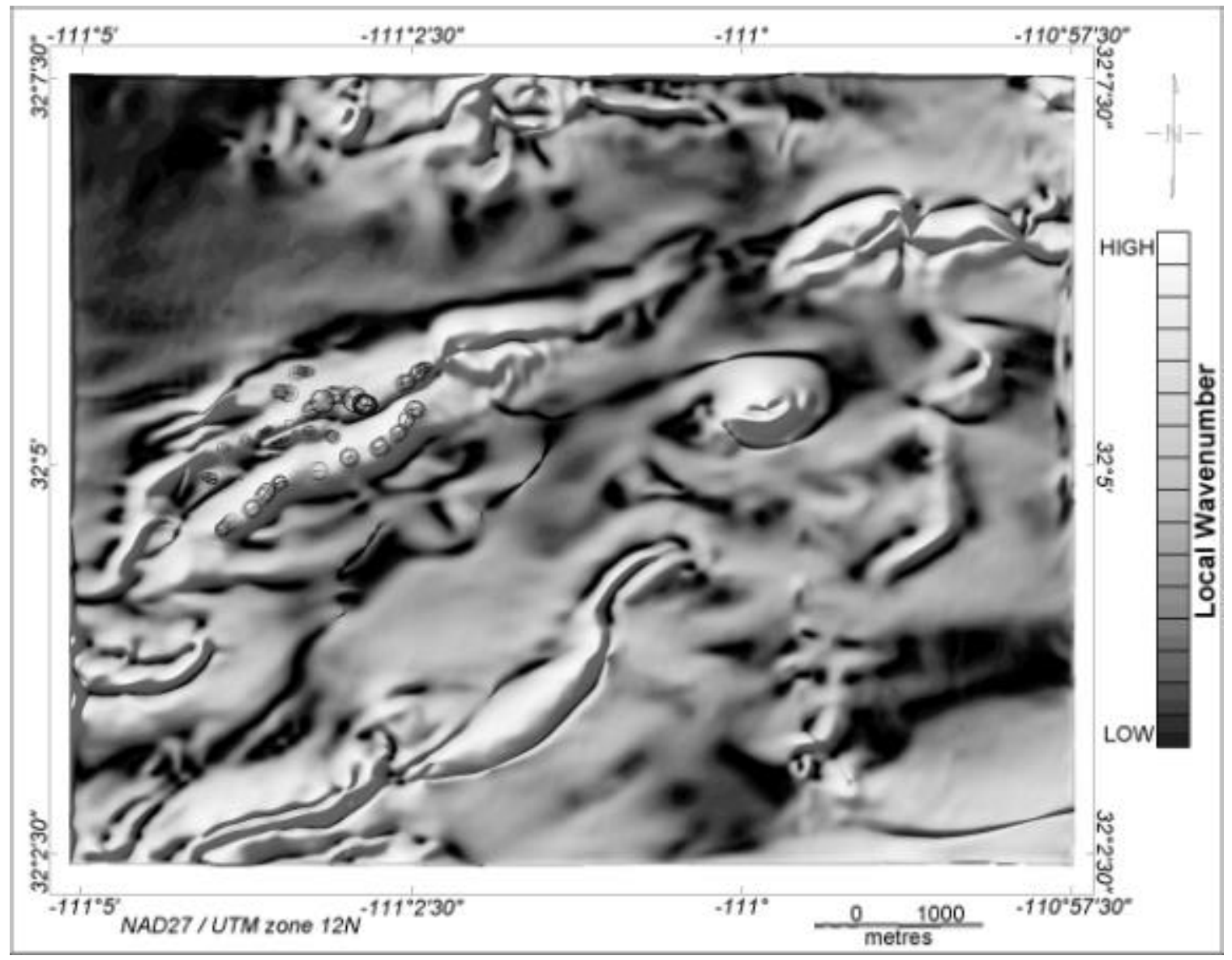

B5. Depth to source map over Black Mountain using the local wavenumber method applied to the vertical integral of the total magnetic field. 


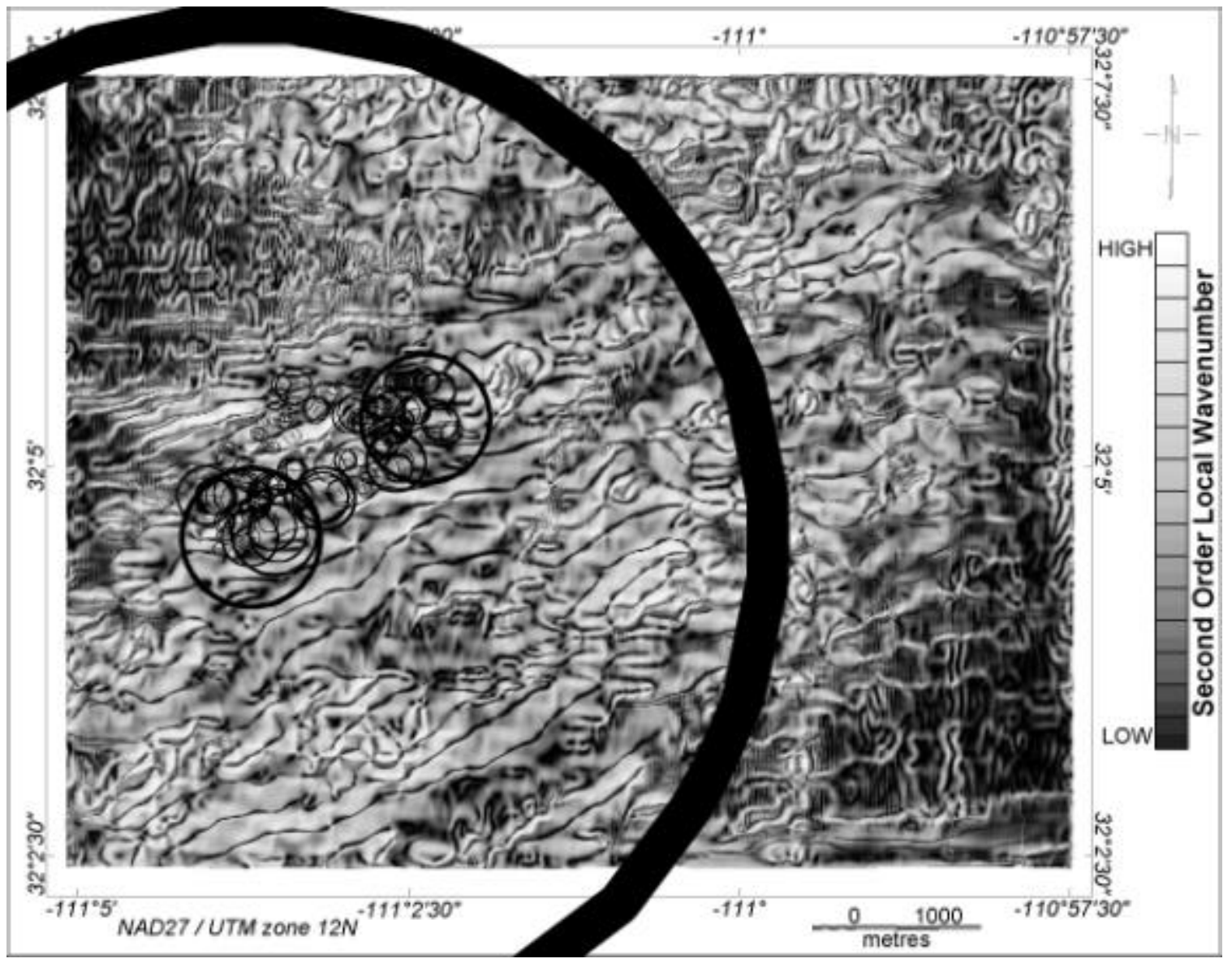

B6. Depth to source map over Black Mountain using the enhanced local wavenumber method. 


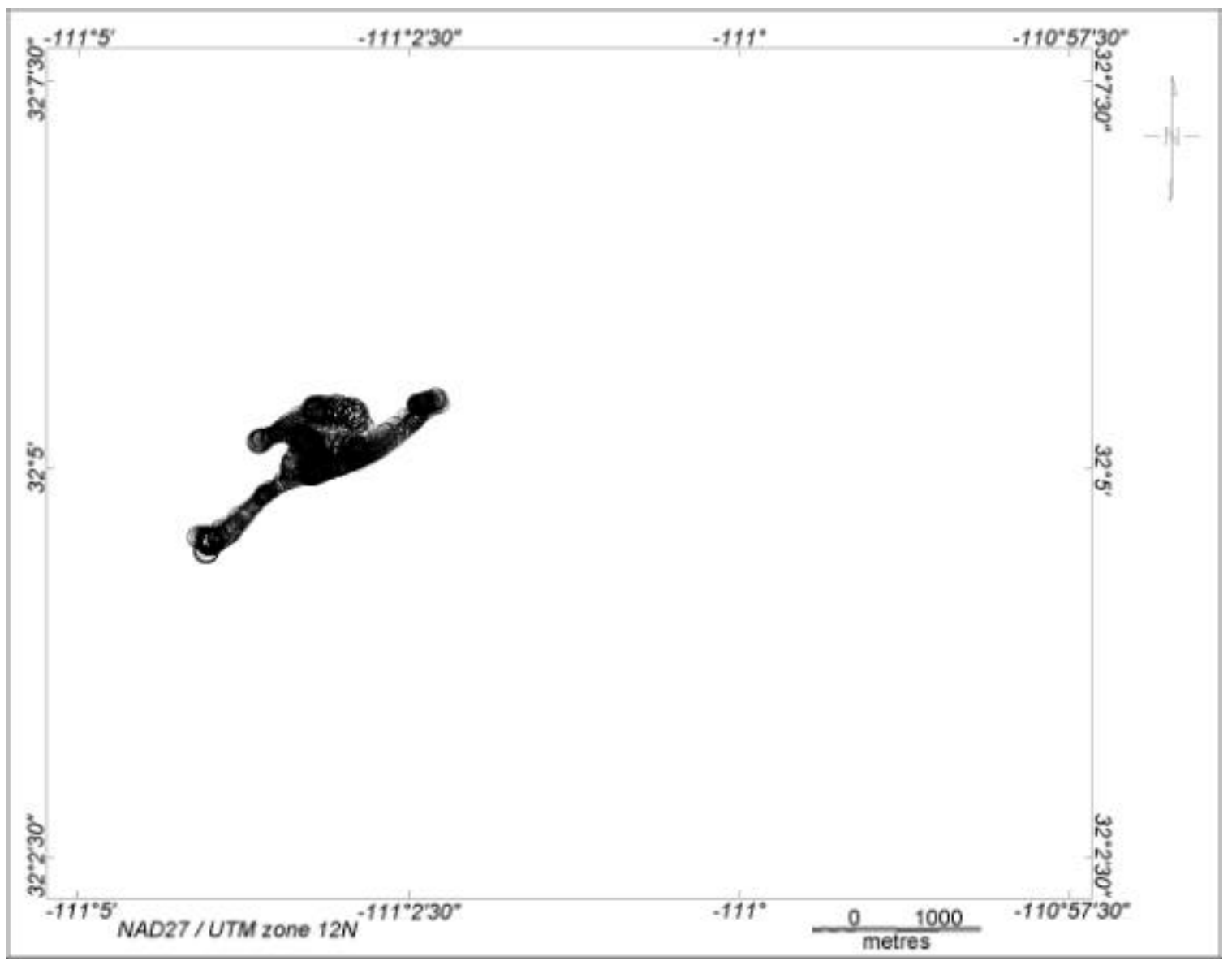

B7. Depth to source map over Black Mountain using Euler deconvolution, for $\mathrm{N}=1$. 


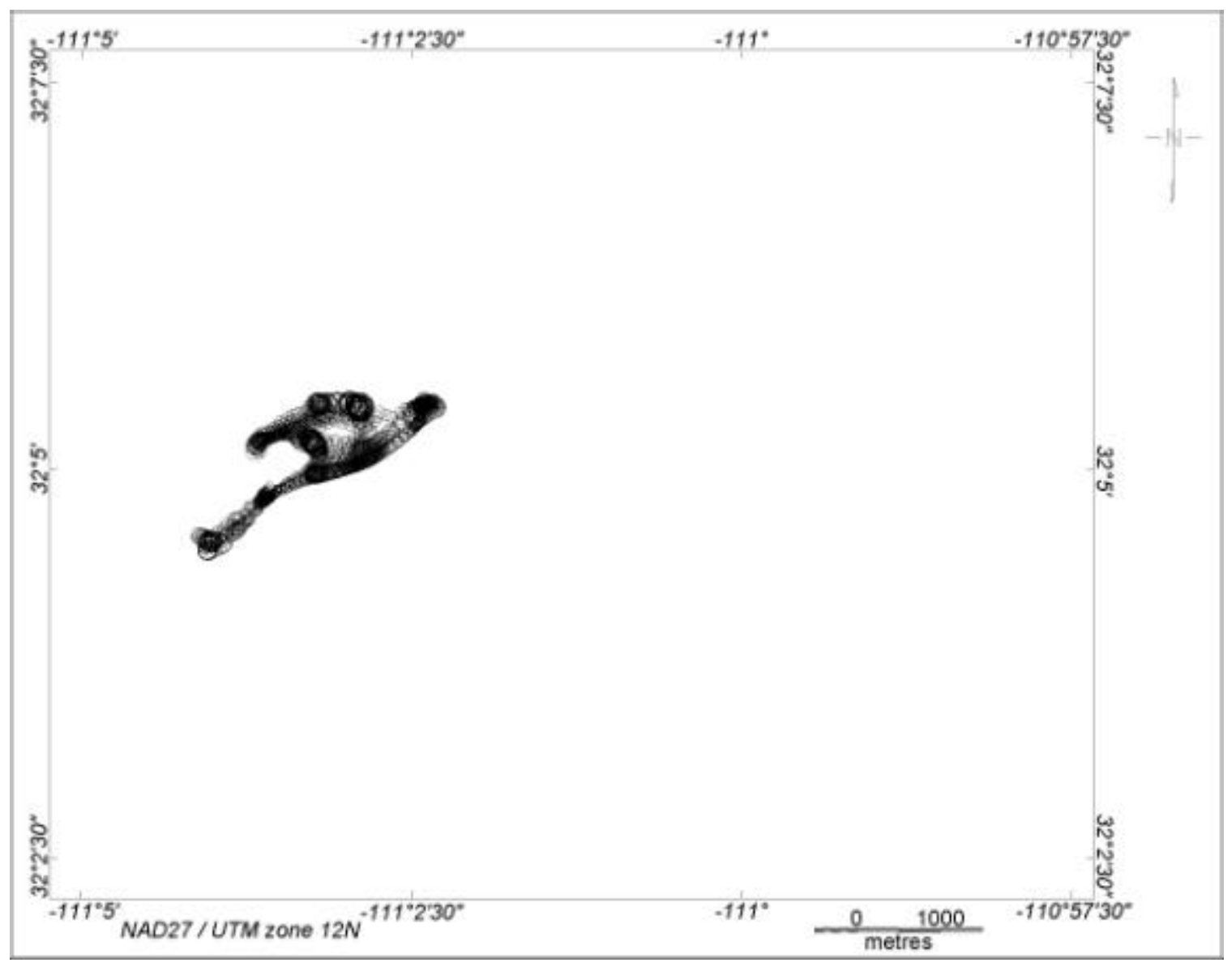

B8. Depth to source map over Black Mountain using Eulerdeconvolution, for $\mathrm{N}=\mathbf{0 . 5}$. 


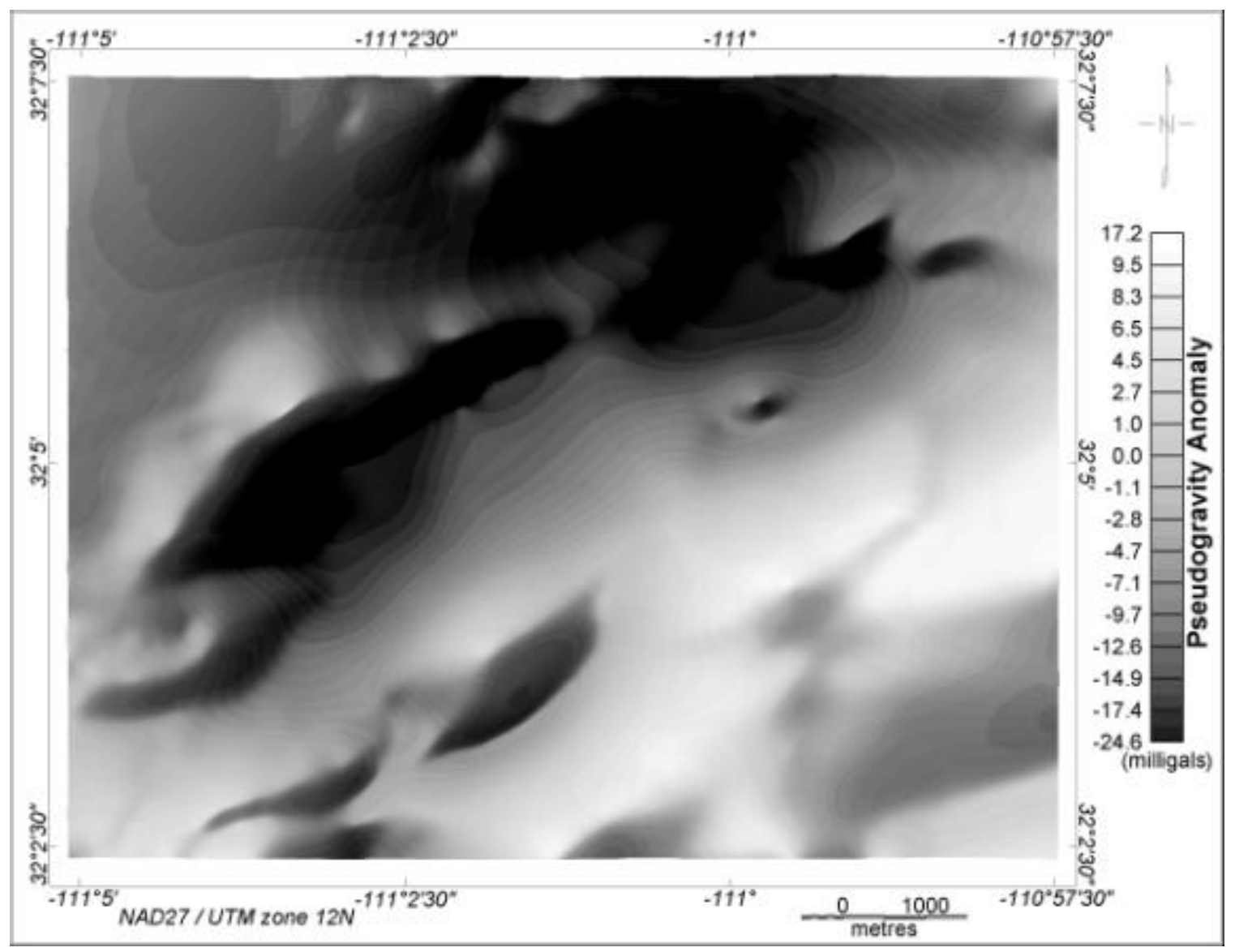

B9. Pseudo-gravity anomaly map over Black Mountain. 


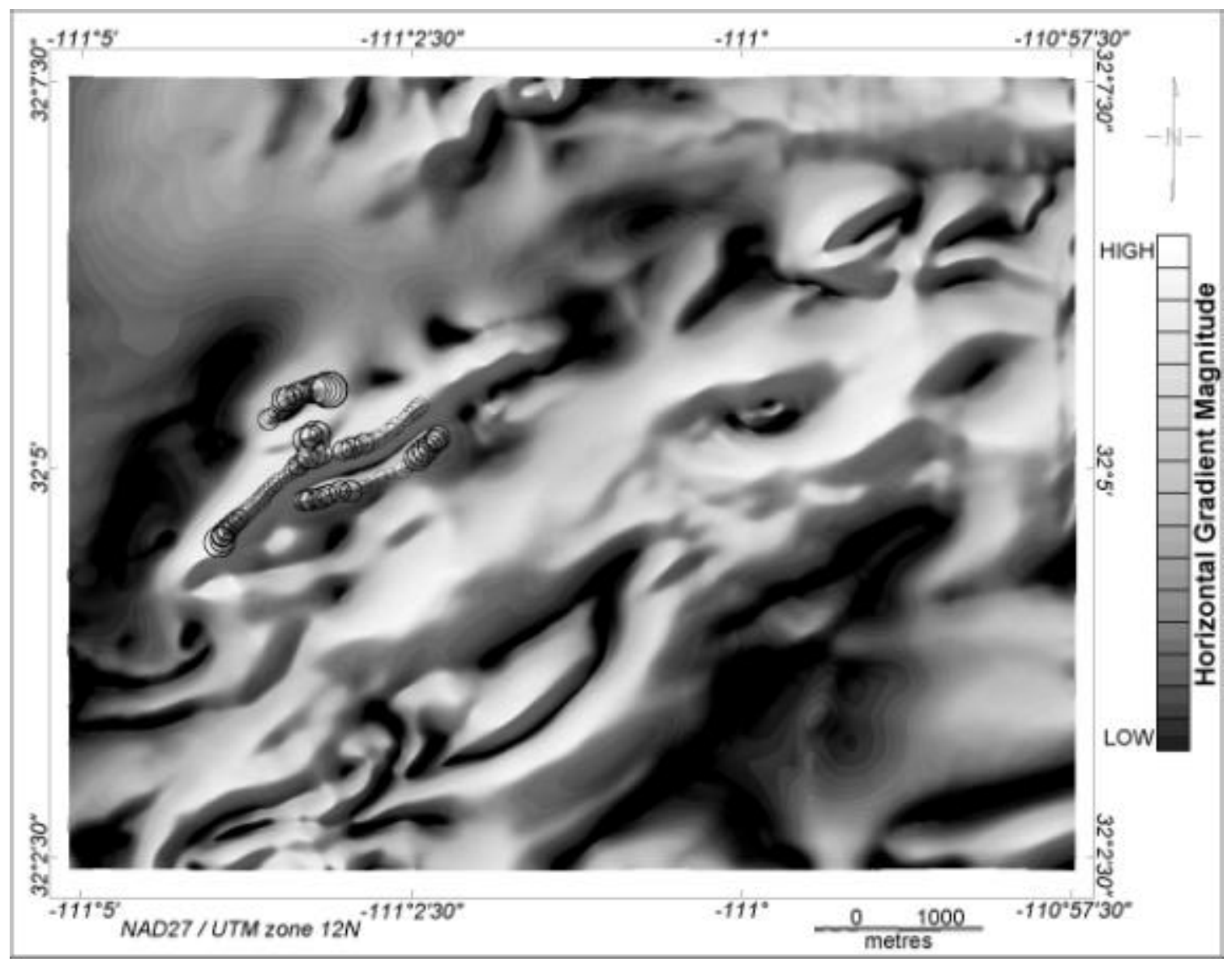

B10. Depth to source map over Black Mountain using the horizontal gradient method applied to the pseudo-gravity field. 


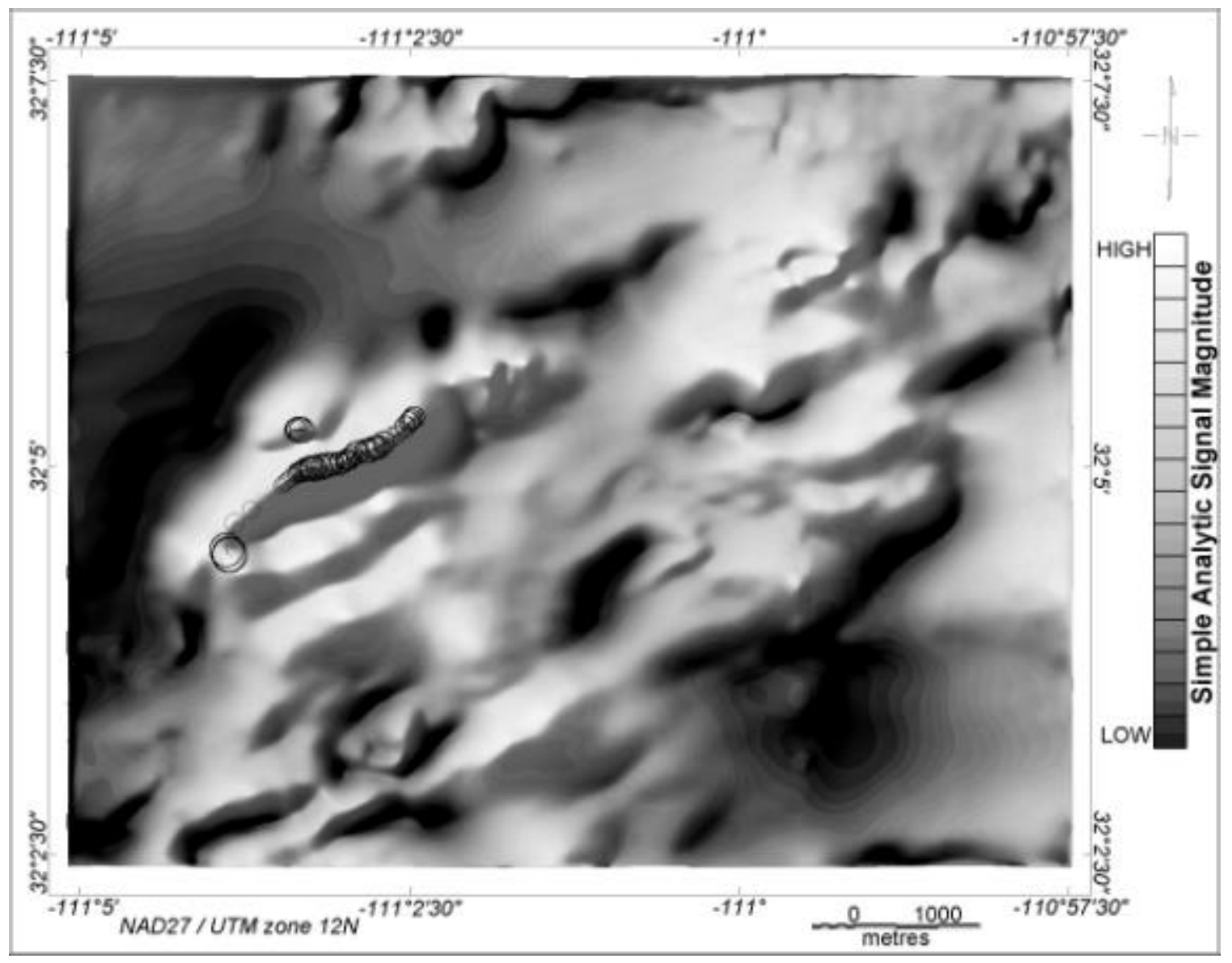

B11. Depth to source map over Black Mountain using the simple analytic signal method applied to the pseudo-gravity field. 


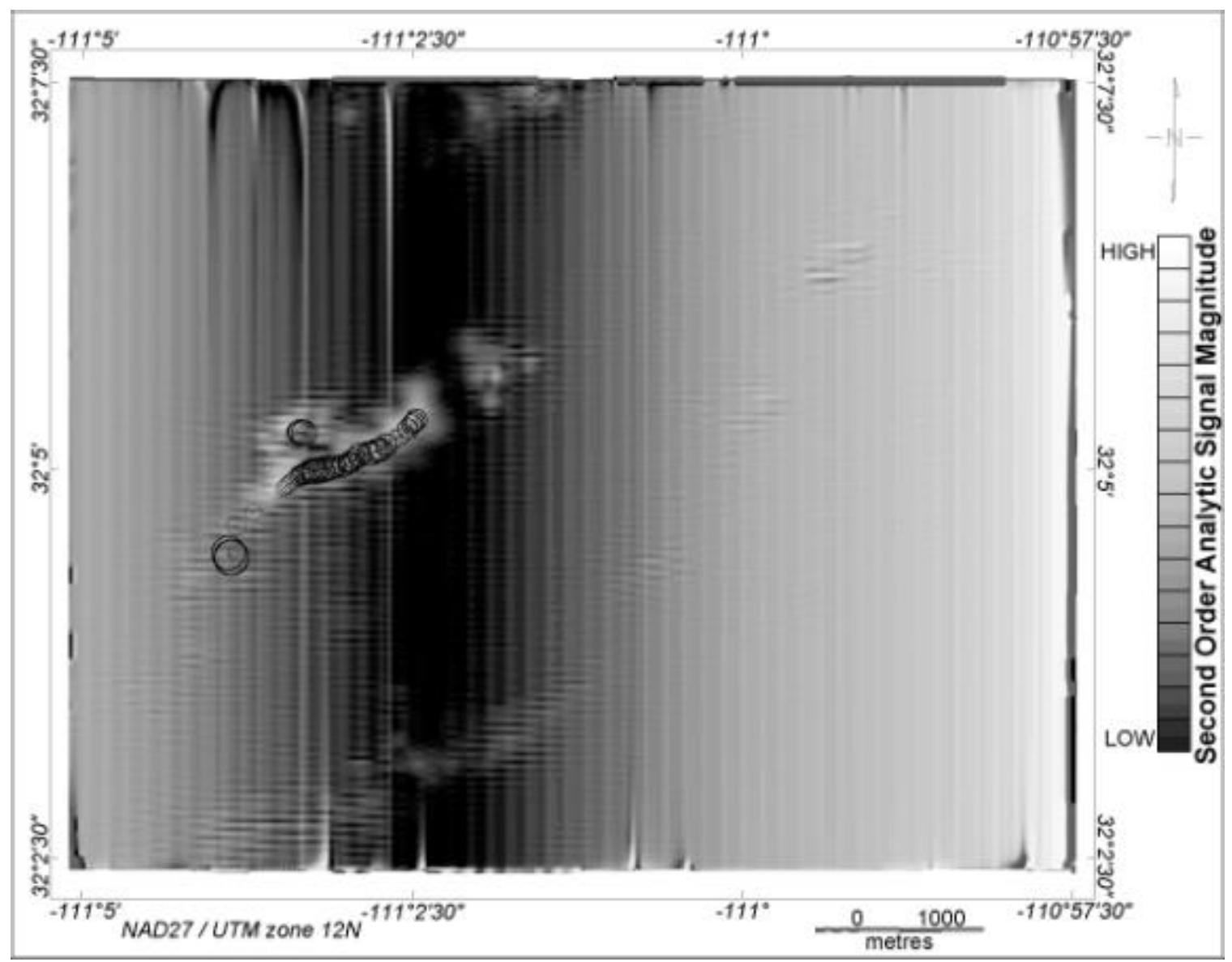

B12. Depth to source map over Black Mountain using the enhanced analytic signal method (step model) applied to the pseudo-gravity field. 


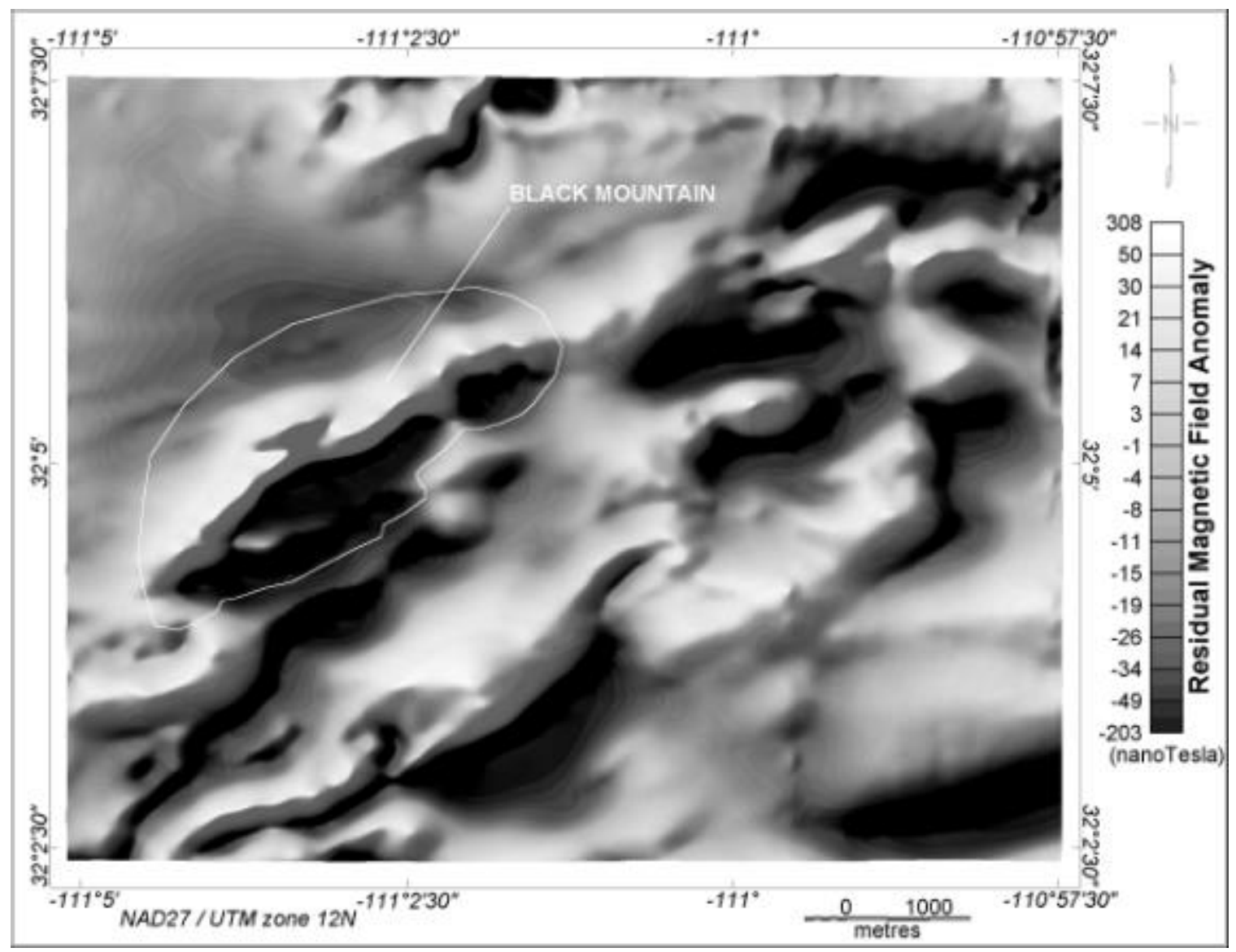

B13. High-pass filtered magnetic field anomaly map over Black Mountain. The white outline borders the topographic expression of Black Mountain. 


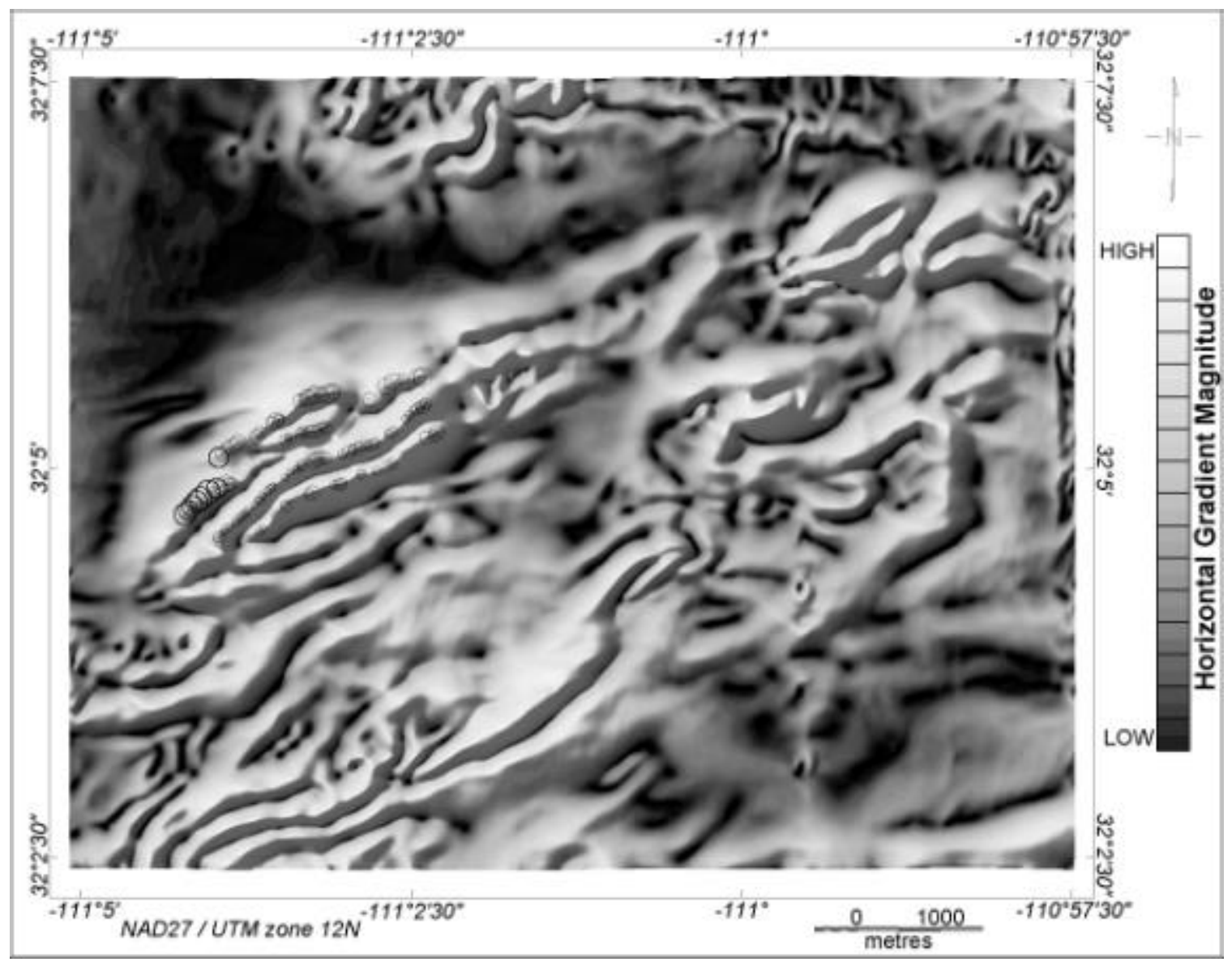

B14. Depth to source map over Black Mountain using the horizontal gradient method applied to the high-pass filtered data. 


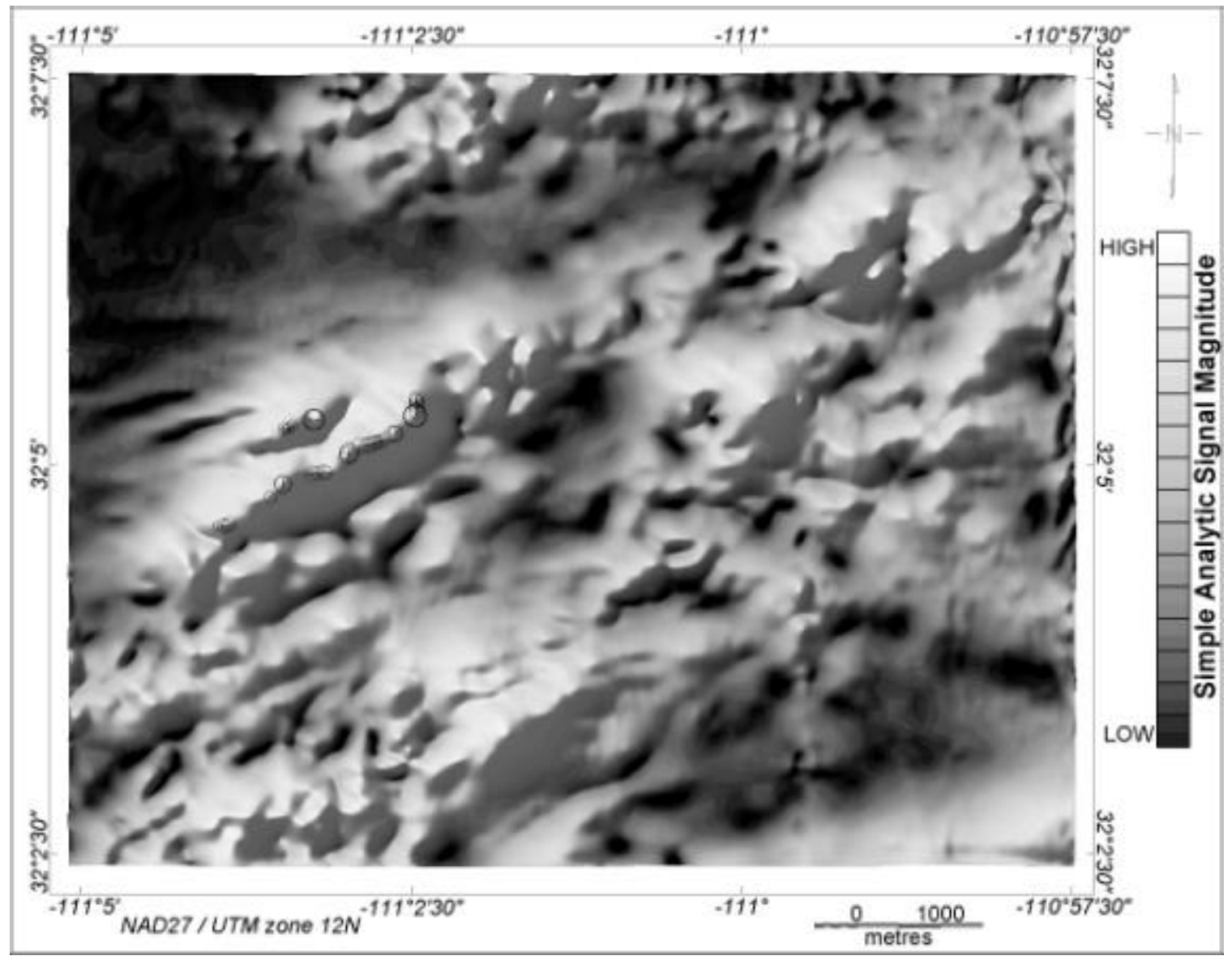

B15. Depth to source map over Black Mountain using the simple analytic signal method applied to the high-pass filtered data. 


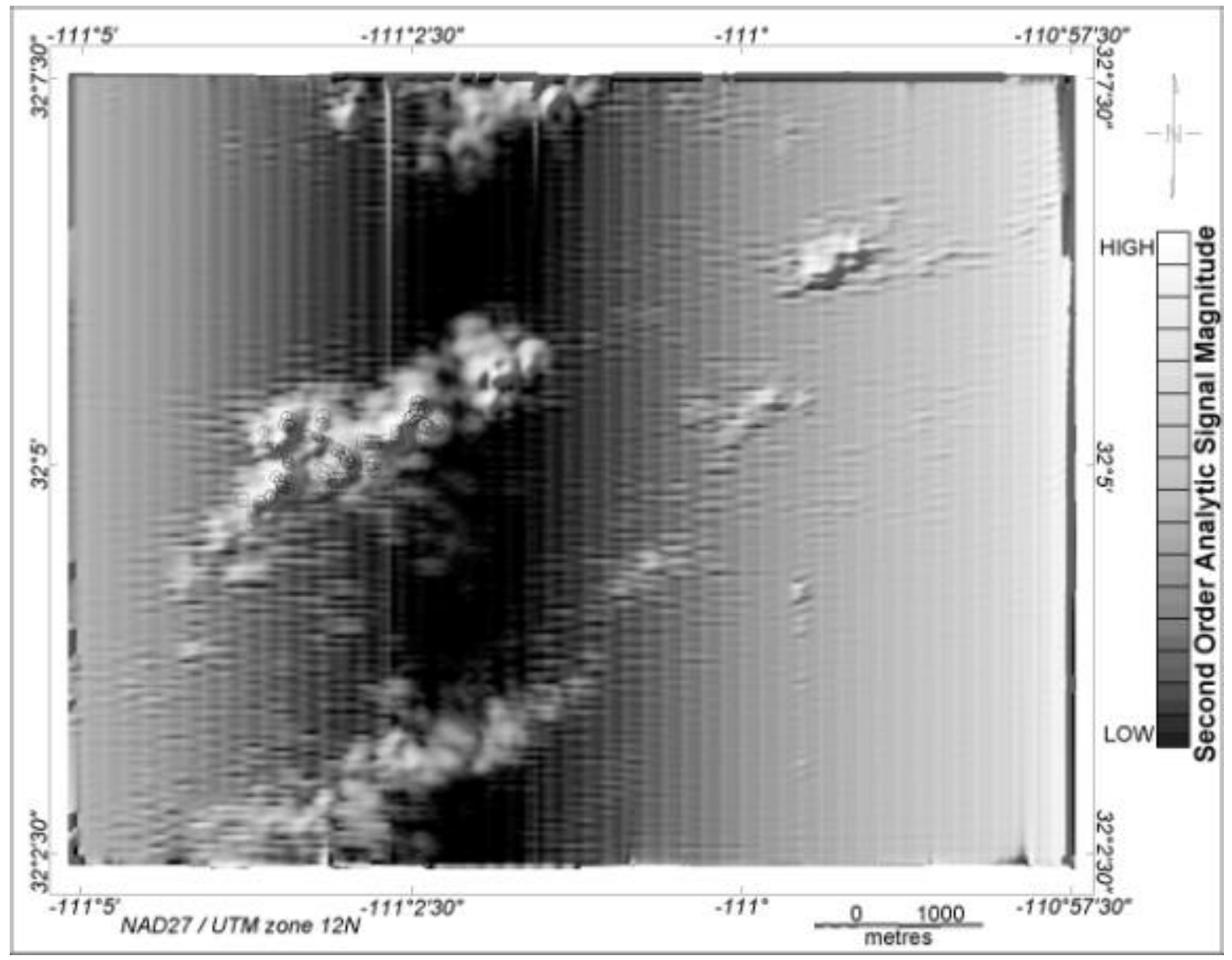

B16. Depth to source map over Black Mountain using the enhanced analytic signal method (step model) applied to the high-pass filtered data. 


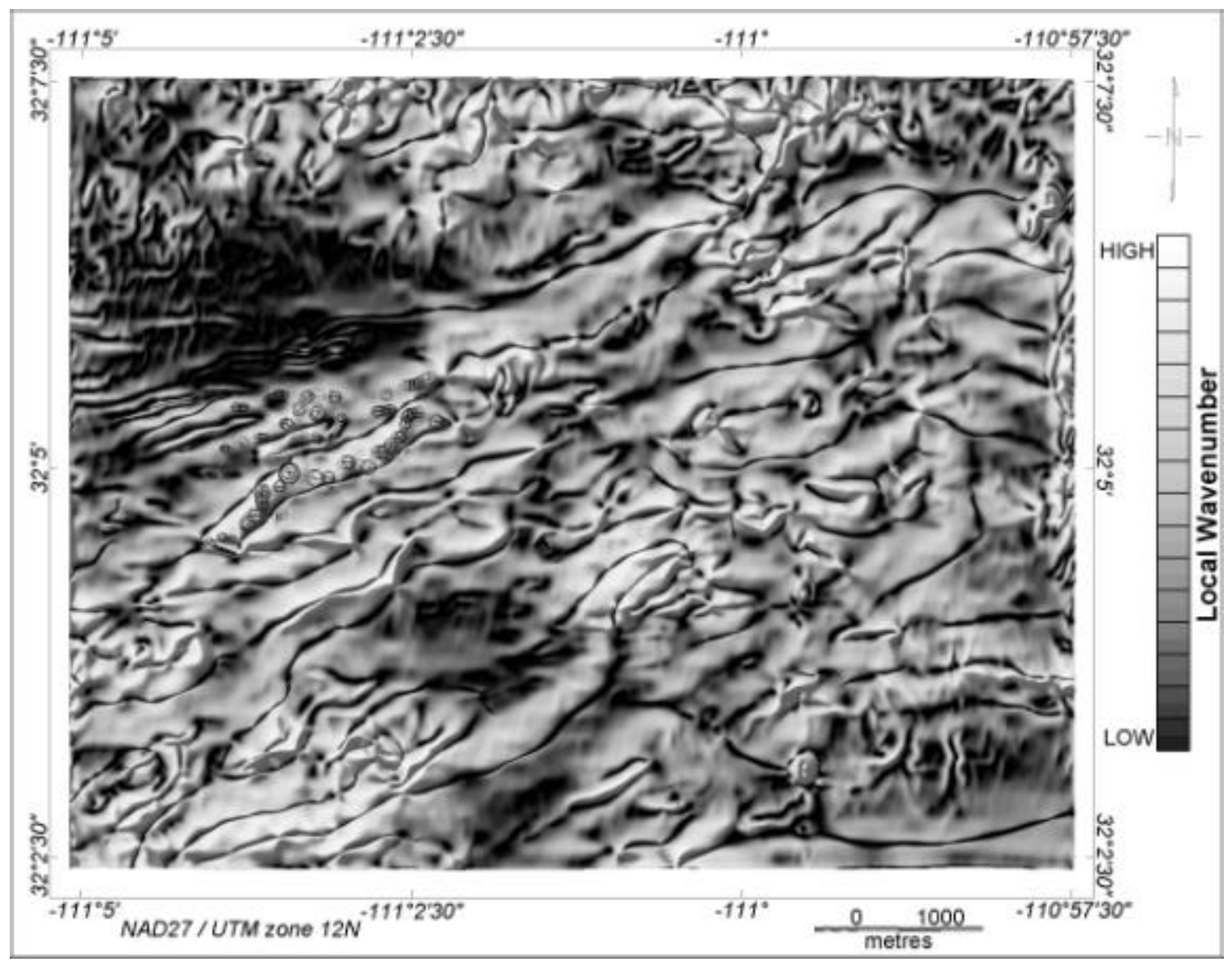

B17. Depth to source map over Black Mountain using the local wavenumber method applied to the high-pass filtered data. 


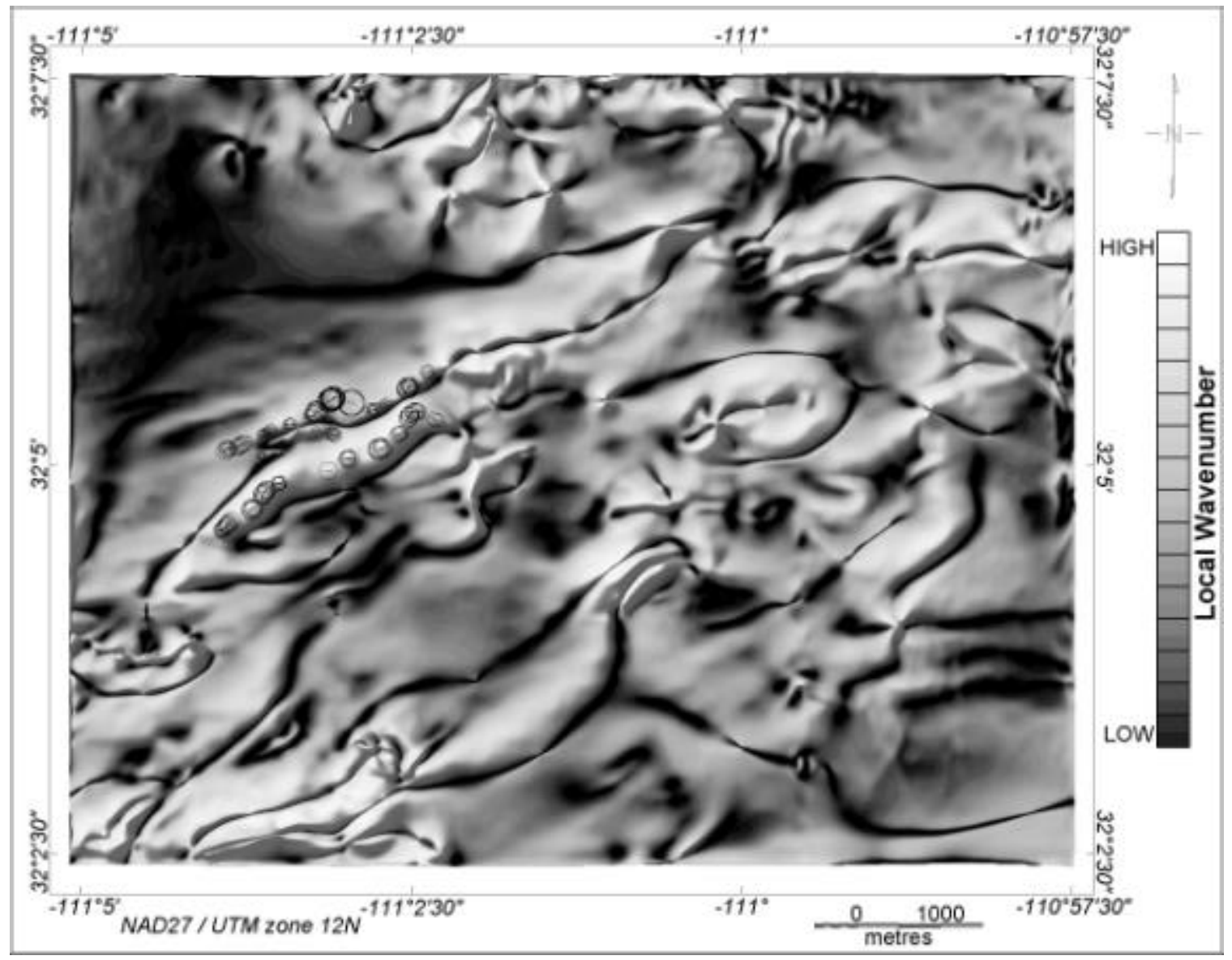

B18. Depth to source map over Black Mountain using the local wavenumber method applied to the vertical integral of the residual magnetic field. 


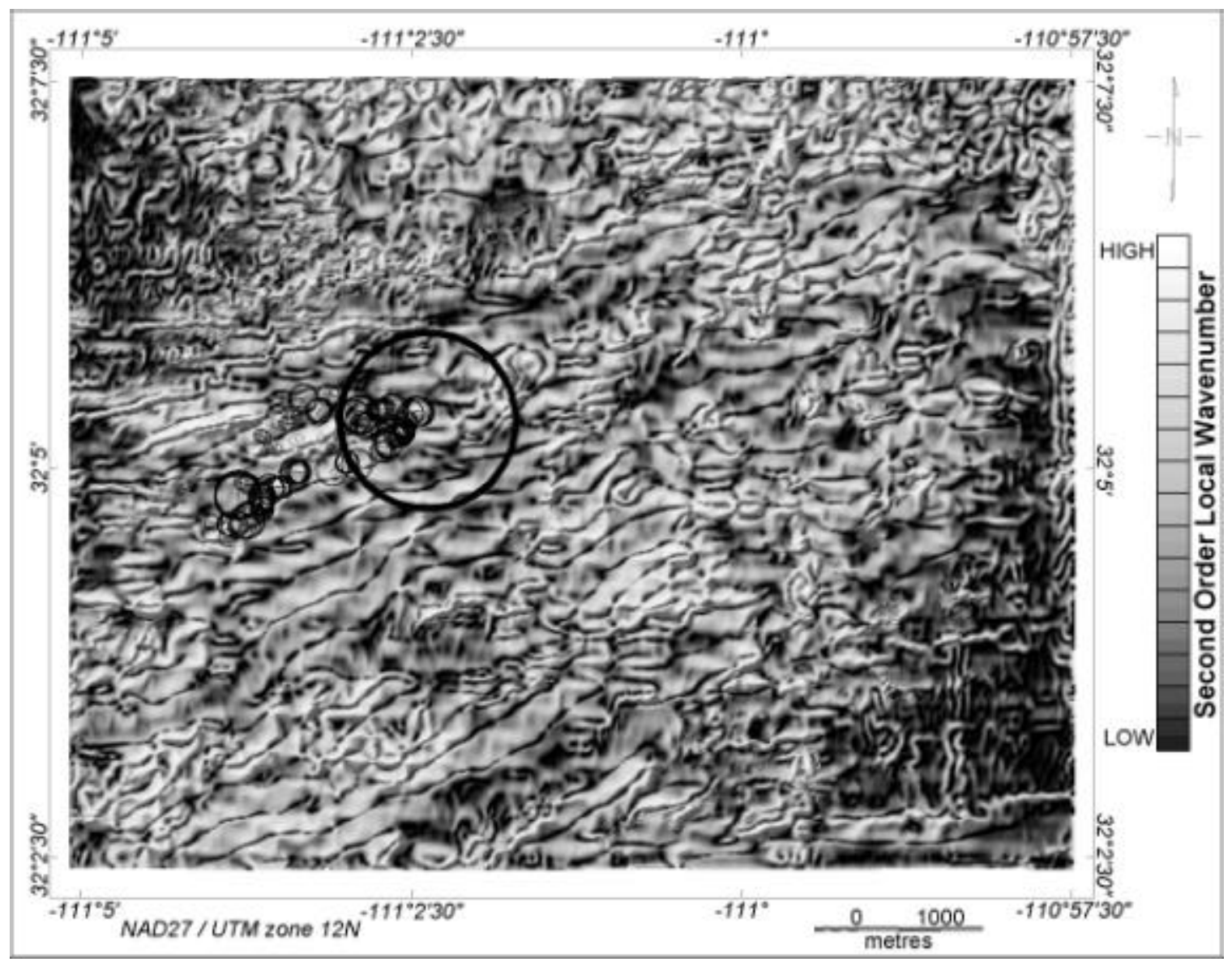

B19. Depth to source map over Black Mountain using the enhanced local wavenumber method applied to the high-pass filtered data. 


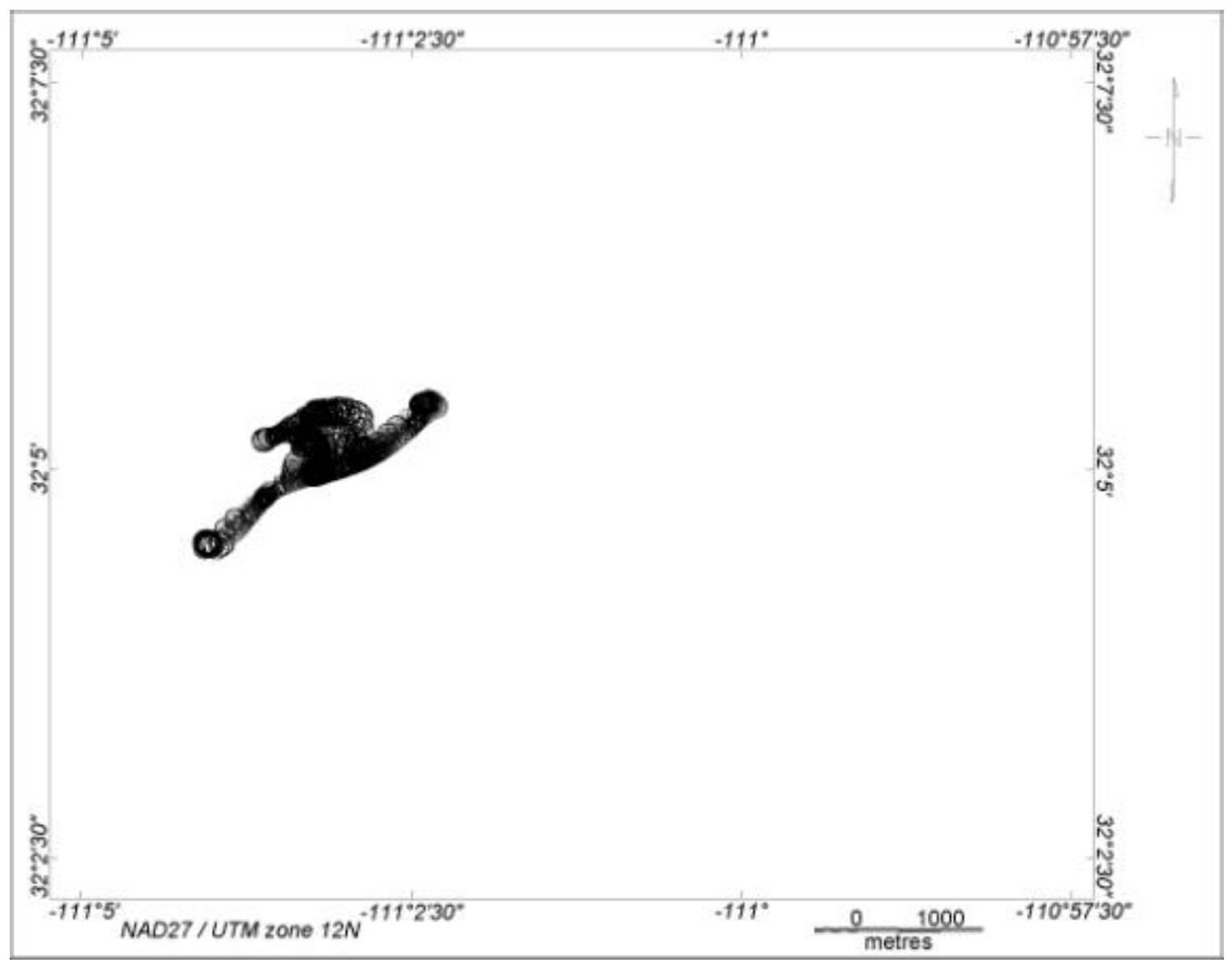

B20. Depth to source map over Black Mountain using Euler deconvolution applied to the high-pass filtered data, for $\mathrm{N}=1$. 


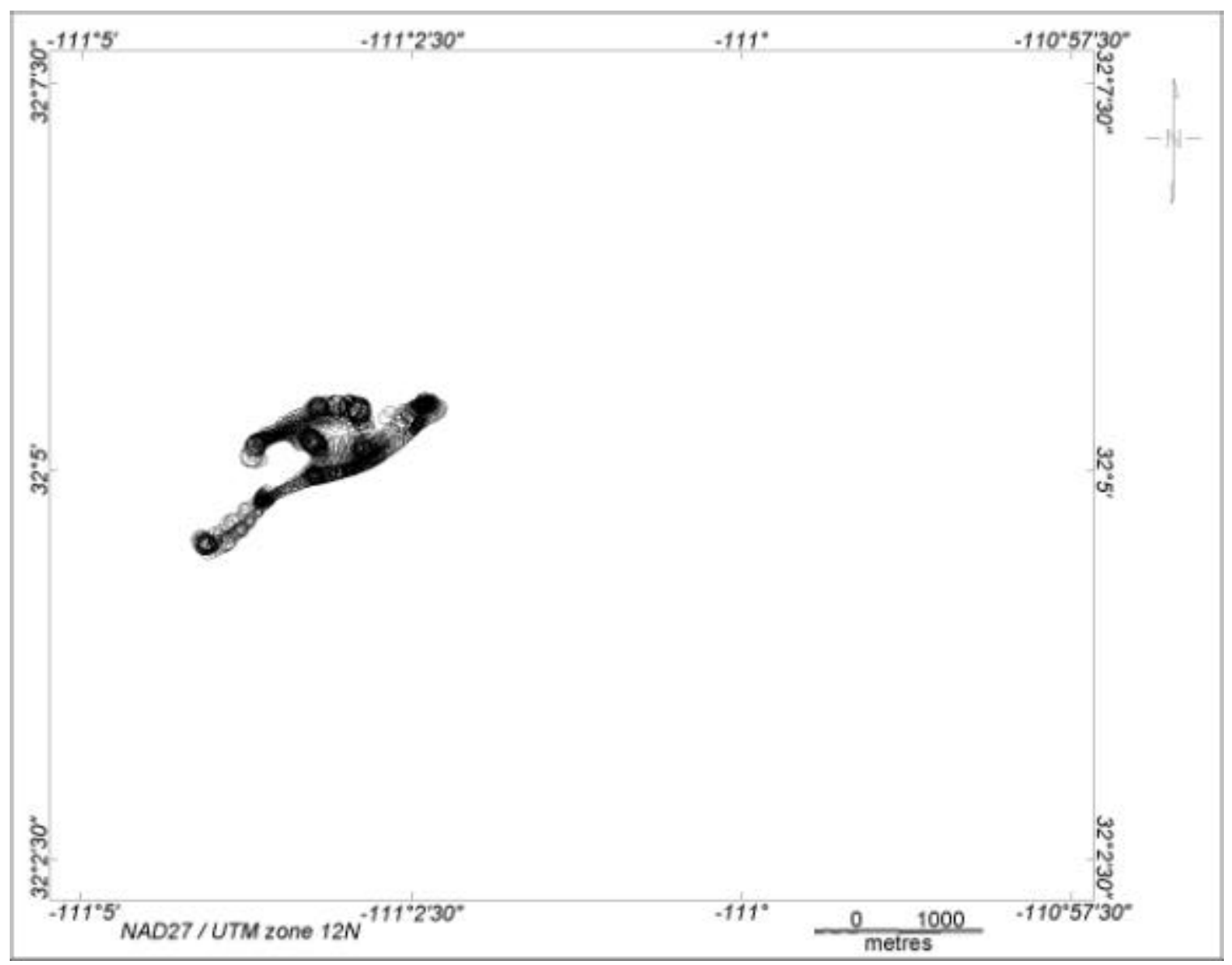

B21. Depth to source map over Black Mountain using Euler deconvolution applied to the high-pass filtered data, for $\mathrm{N}=\mathbf{0 . 5}$. 


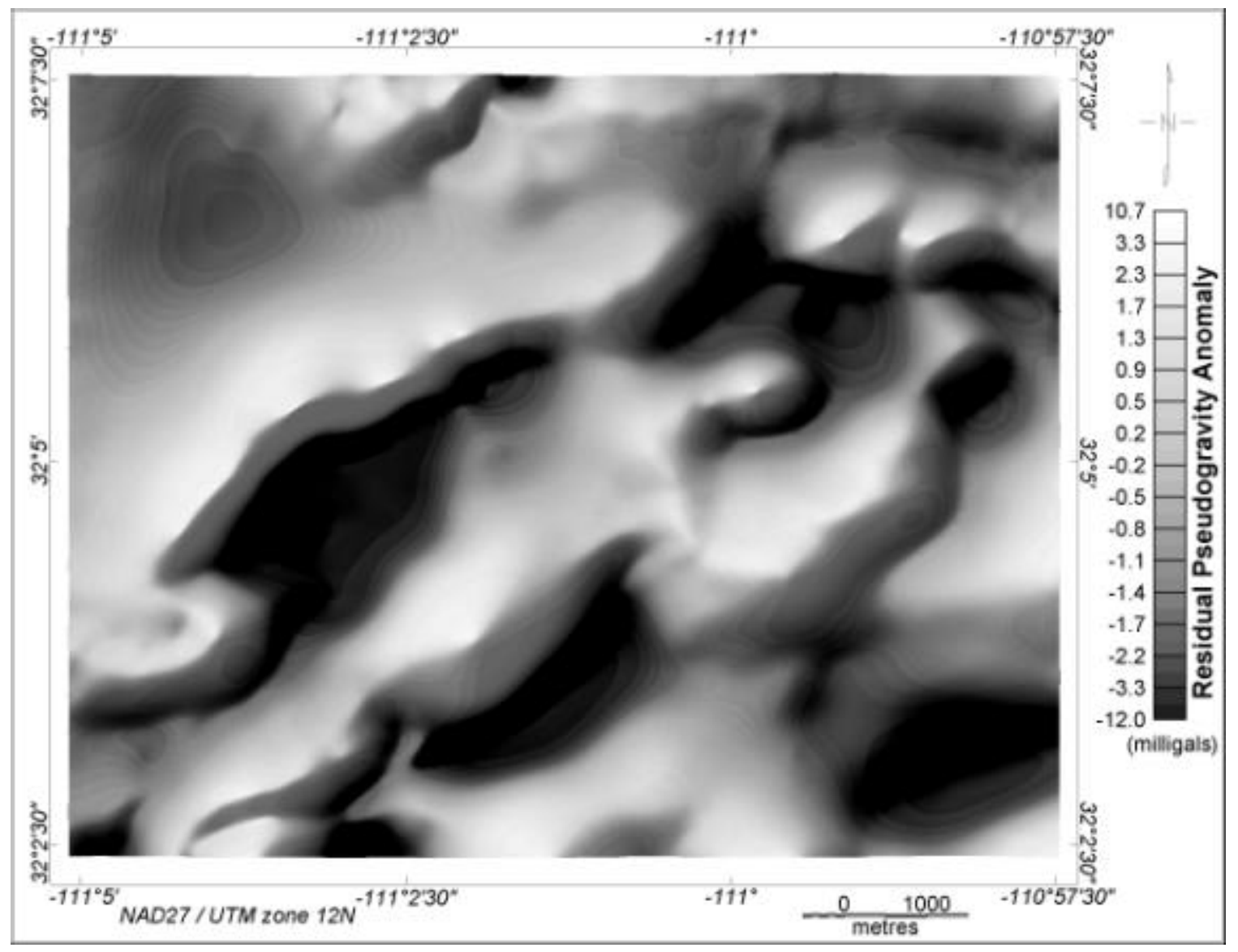

B22. High-pass filtered pseudo-gravity anomaly map over Black Mountain. 


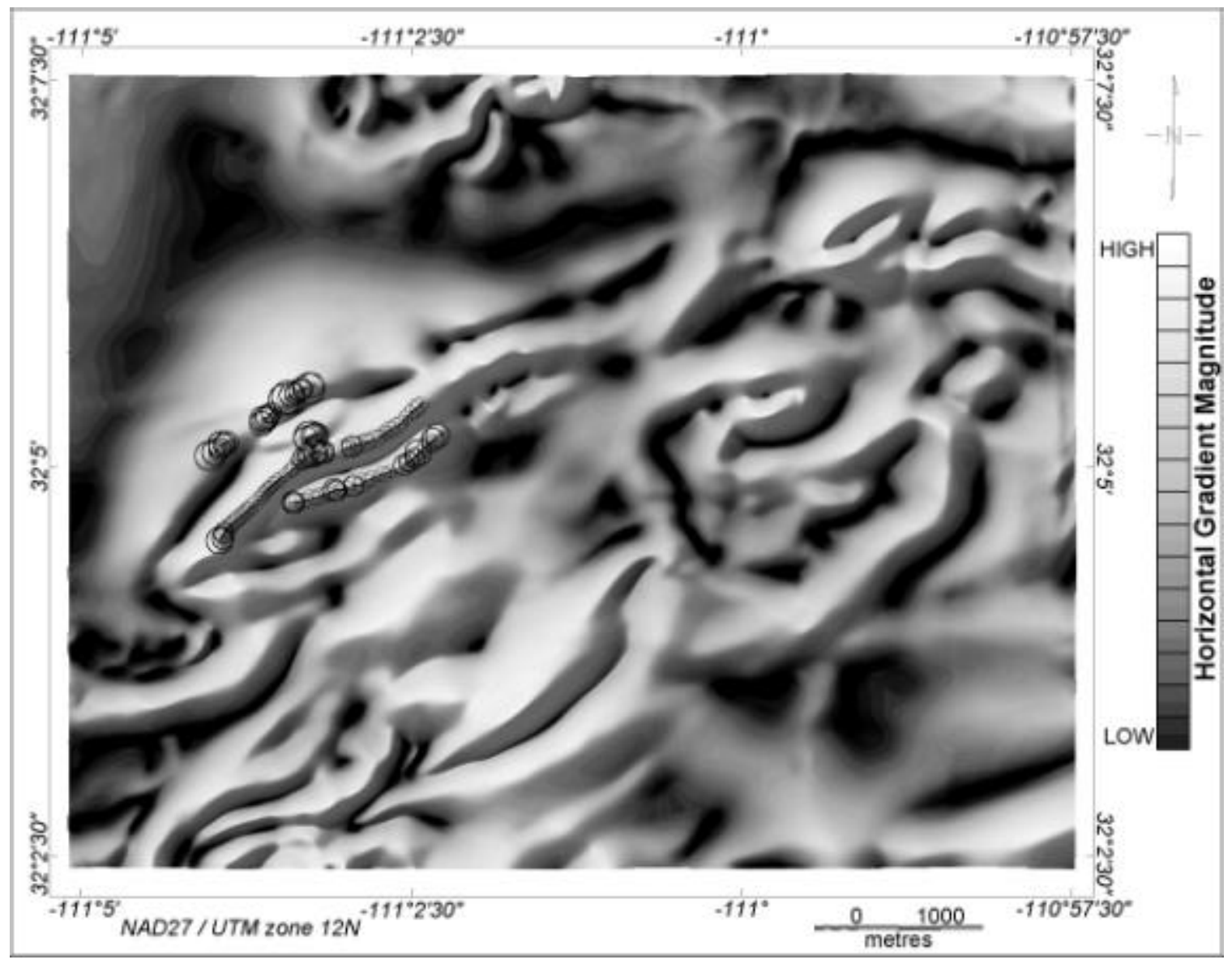

B23. Depth to source map over Black Mountain using the horizontal gradient method applied to the high-pass filtered pseudo-gravity field. 


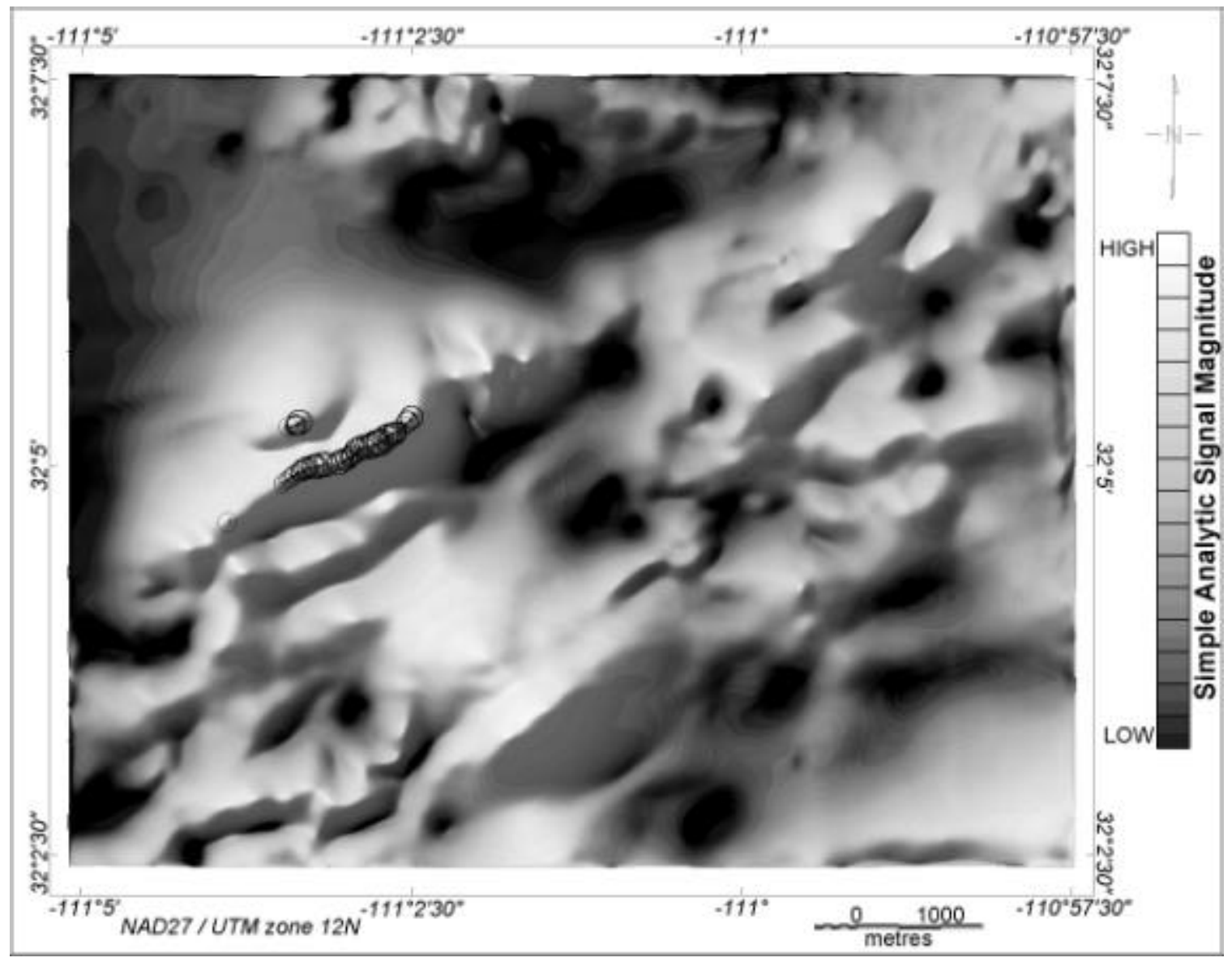

B24. Depth to source map over Black Mountain using the simple analytic signal method applied to the high-pass filtered pseudo-gravity field. 


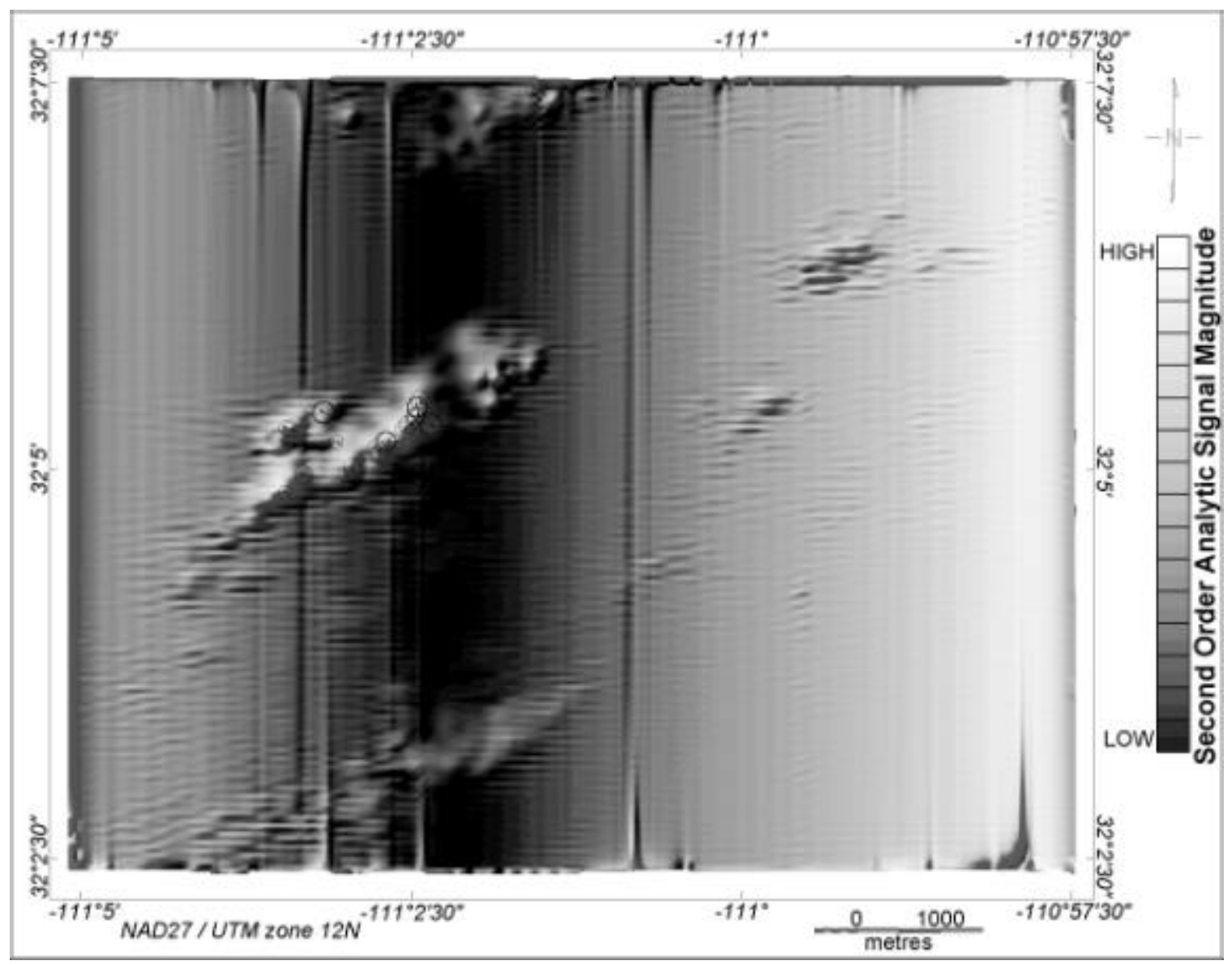

B25. Depth to source map over Black Mountain using the enhanced analytic signal method (step model) applied to the high-pass filtered pseudo-gravity field. 
APPENDIX C: Depth-to-source maps over the natural gas pipelines

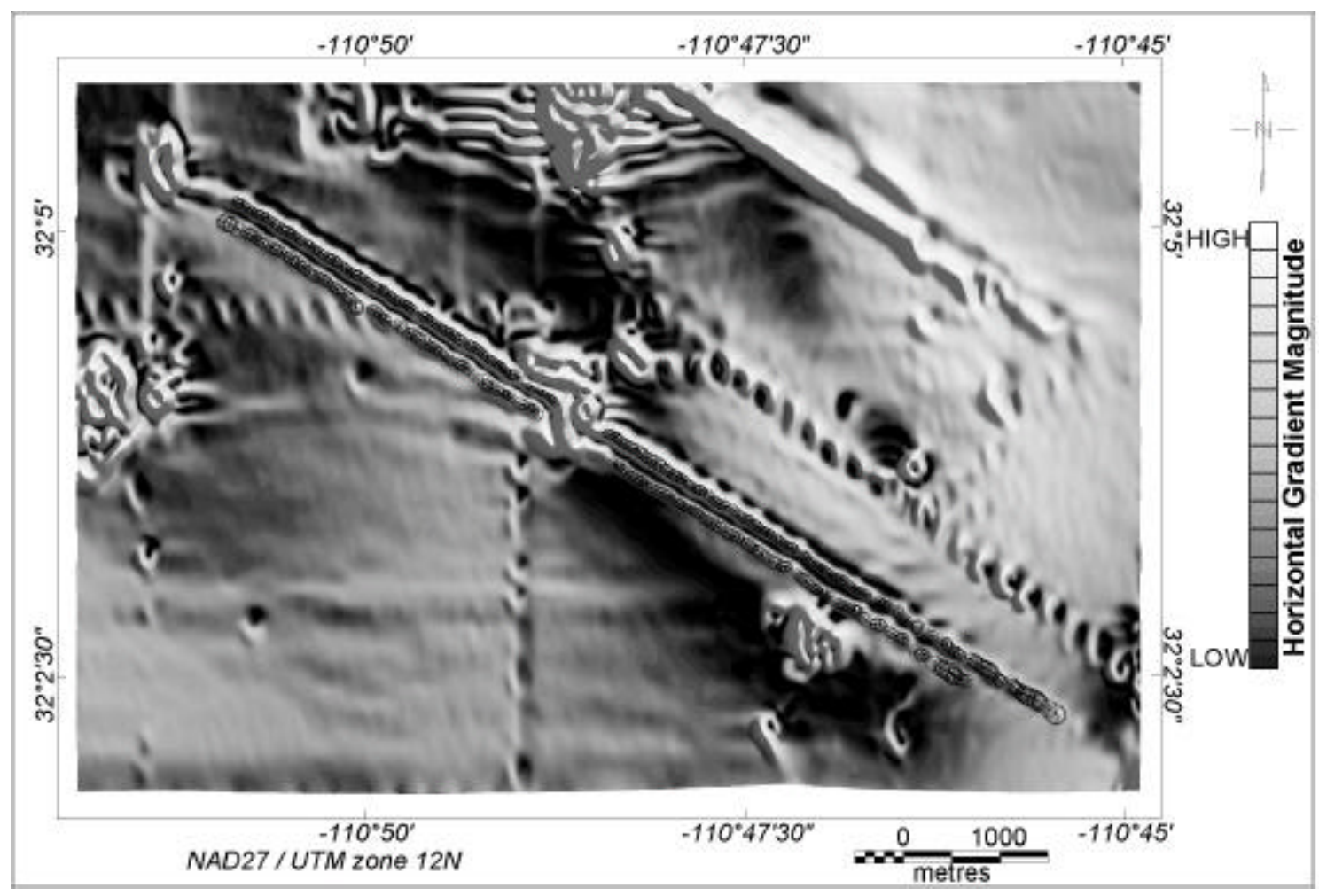

C1. Depth to source map over the pipelines using the horizontal gradient method. The circles are centered over the contacts and their sizes are relative to the depth from the airplane. 


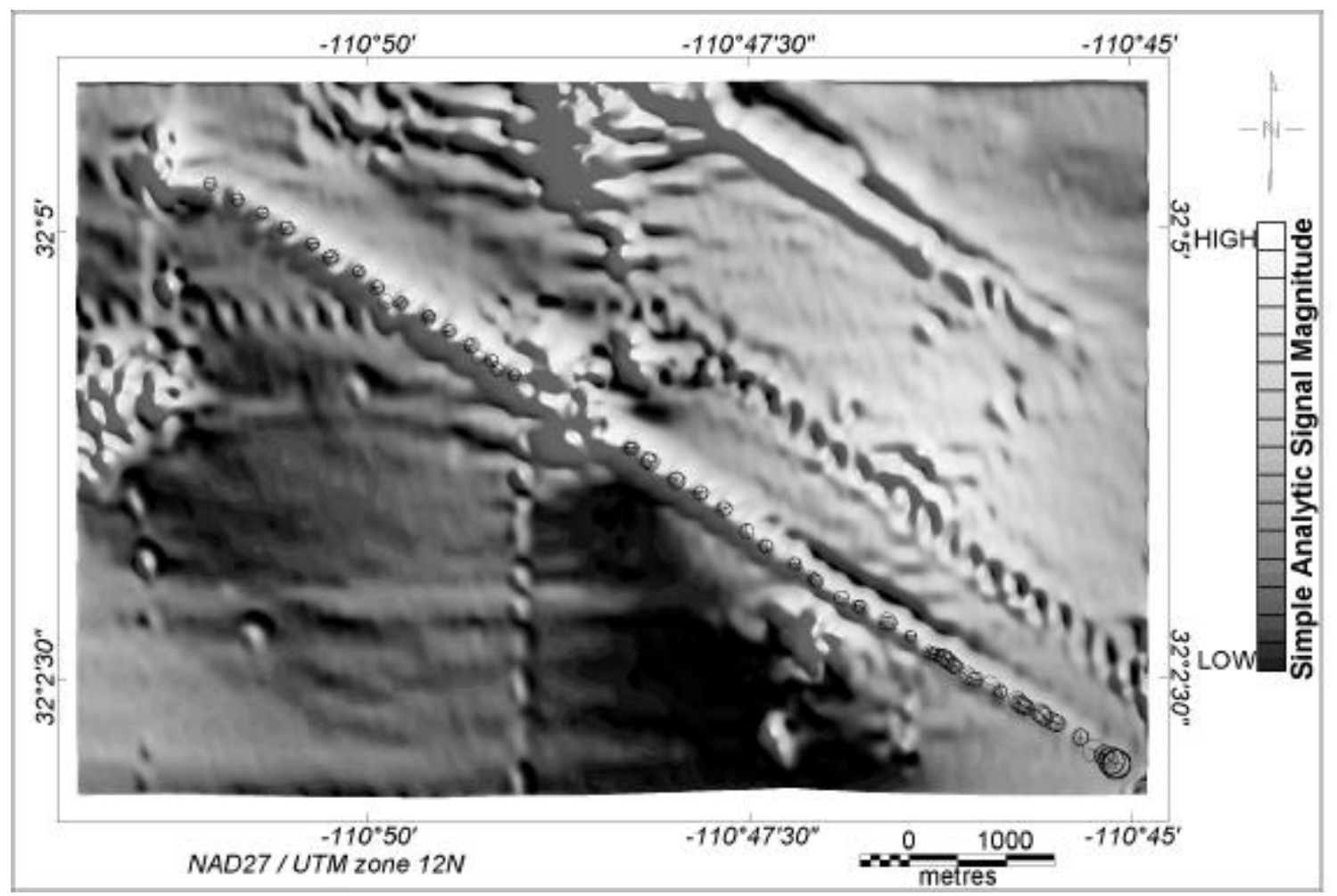

C2. Depth to source map over the pipelines using the simple analytic signal method. 


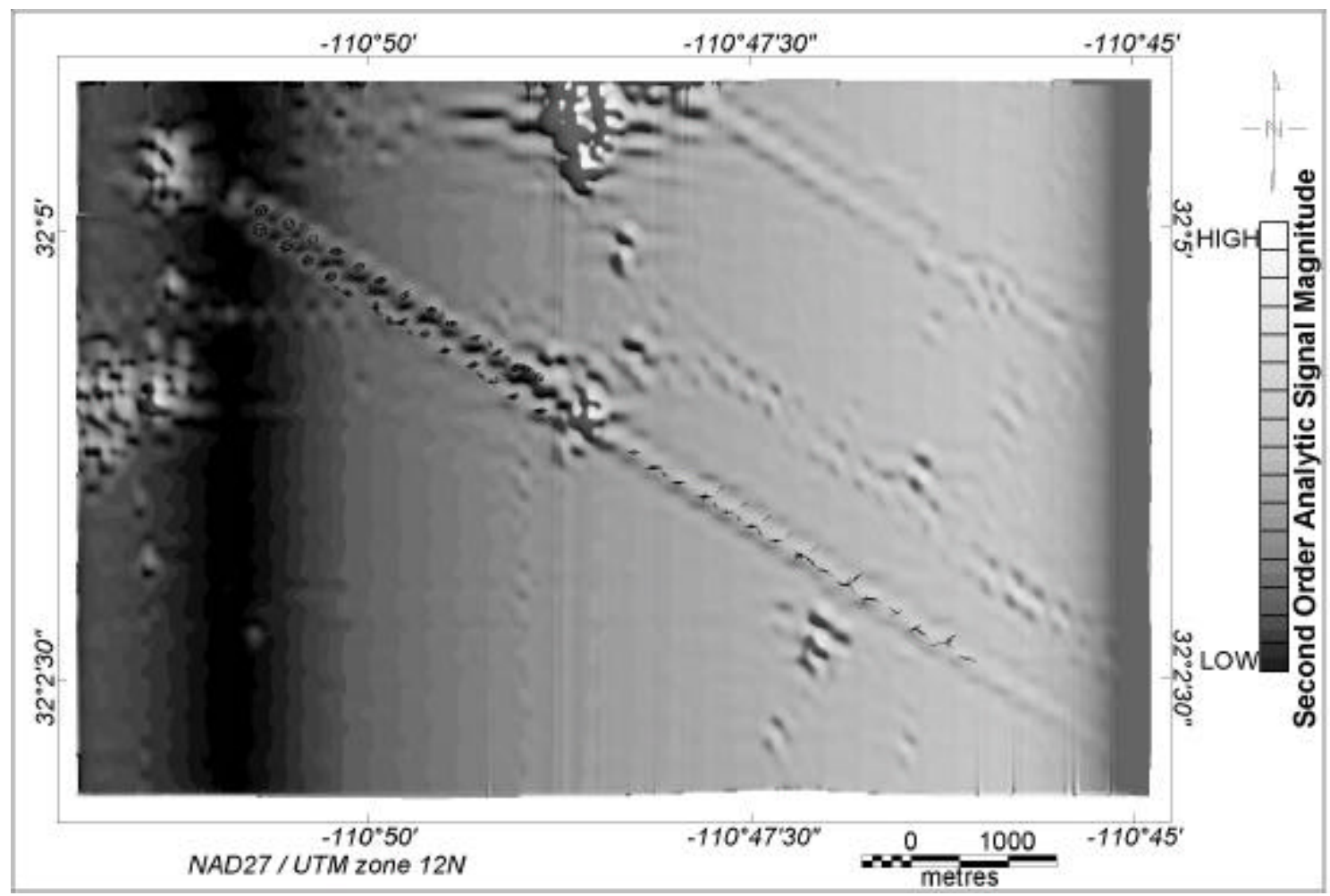

C3. Depth to source map over the pipelines using the enhanced analytic signal method (step model). 


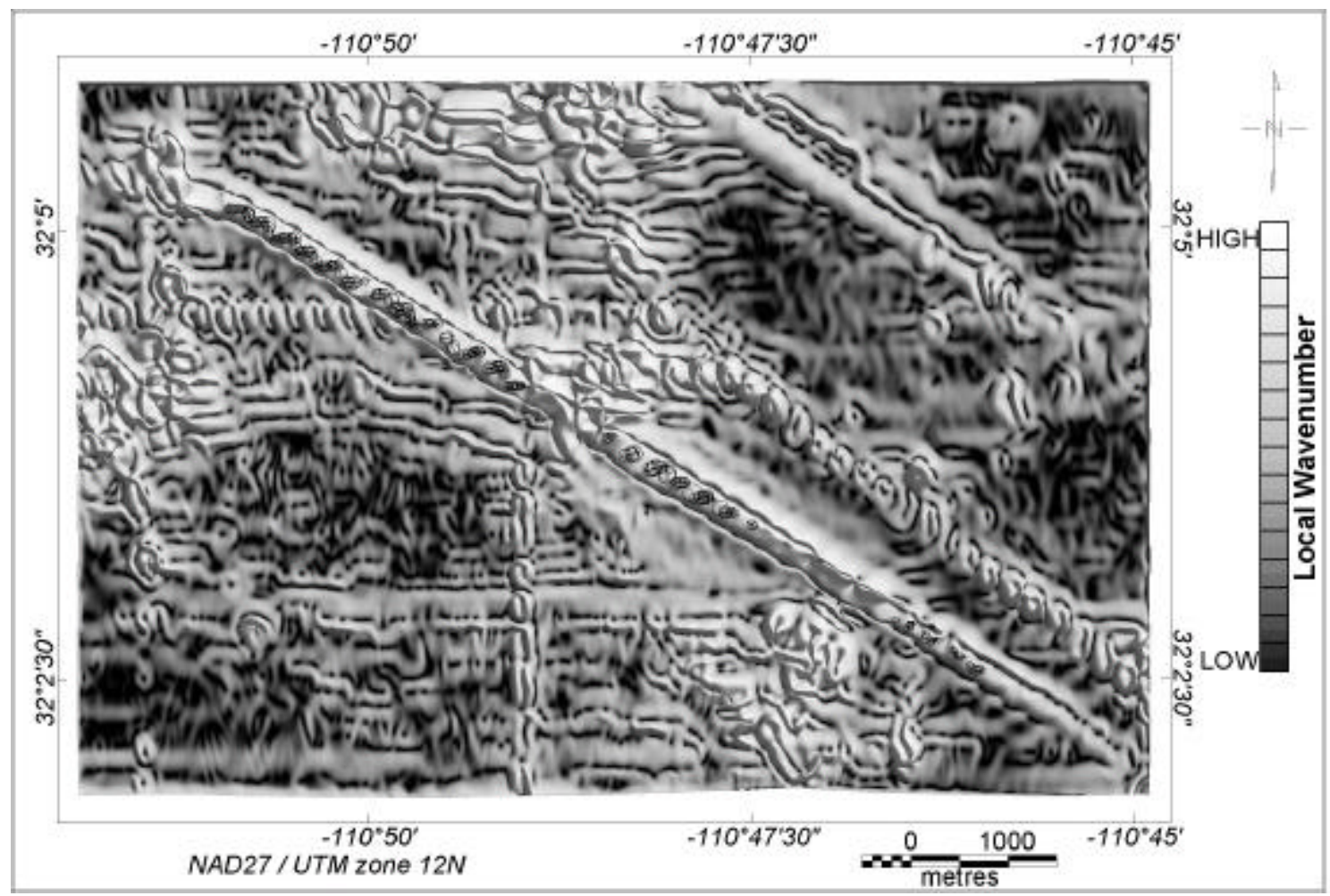

C4. Depth to source map over the pipelines using the local wavenumber method. 


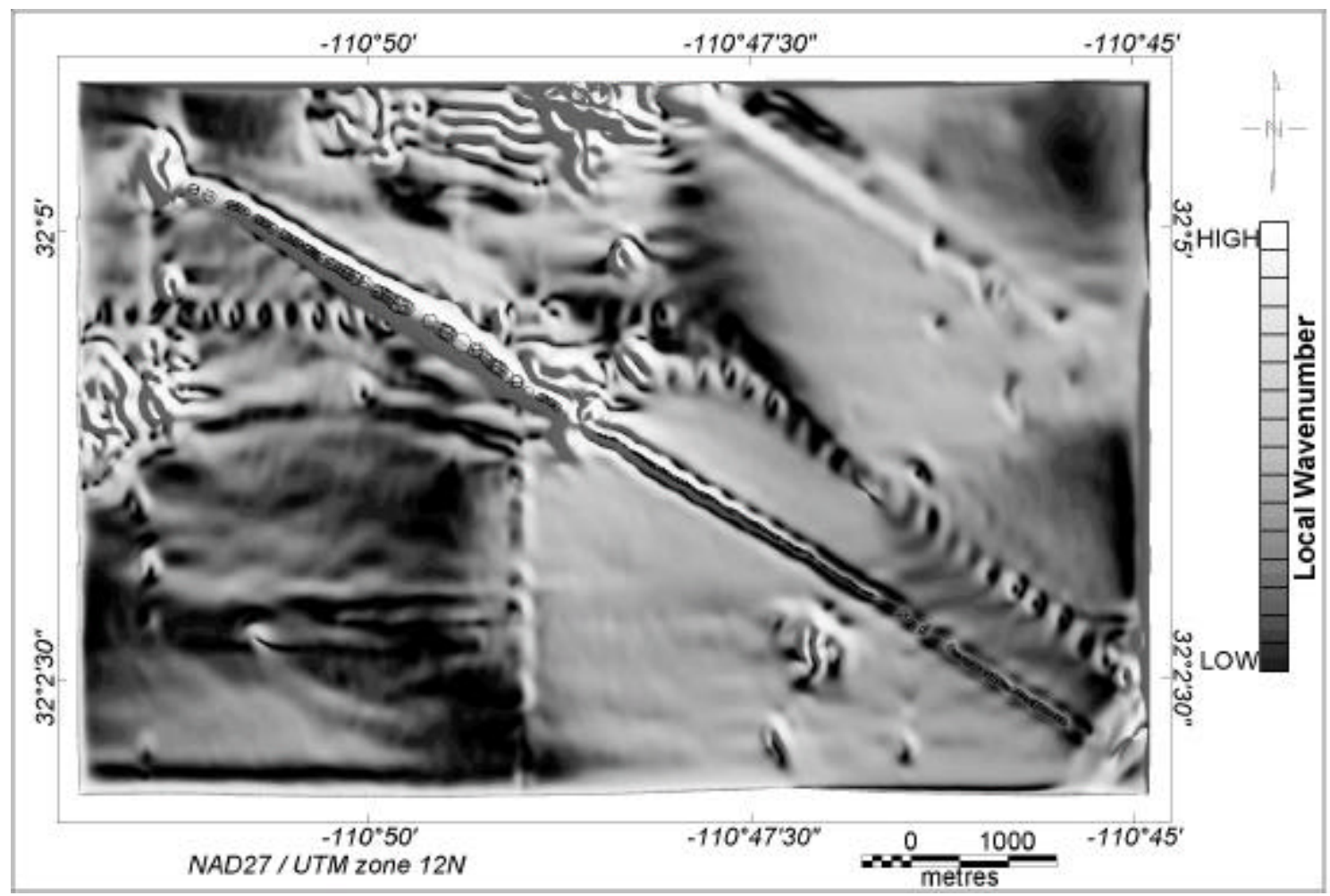

C5. Depth to source map over the pipelines using the local wavenumber method applied to the vertical integral of the total magnetic field. 


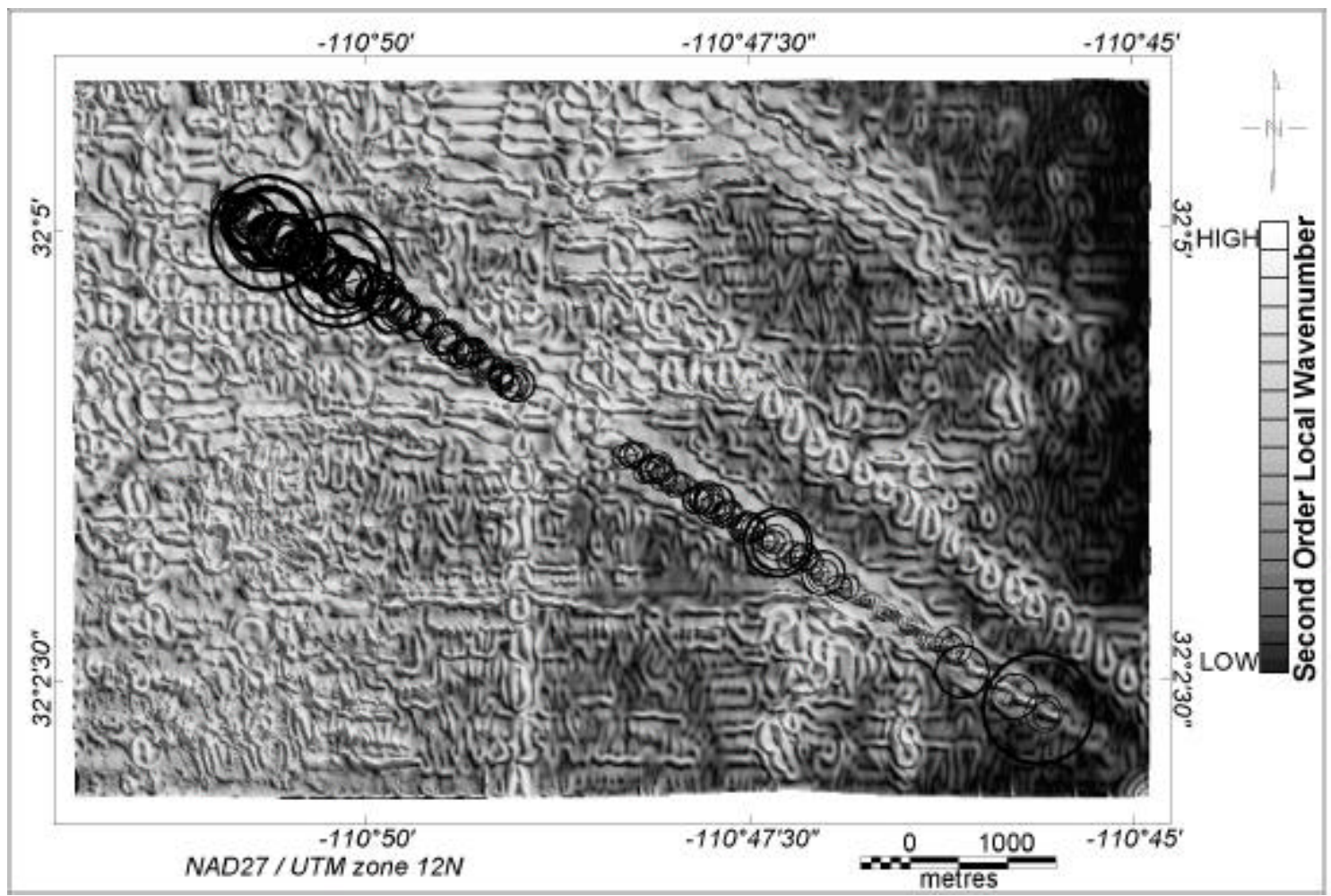

C6. Depth to source map over the pipelines using the enhanced local wavenumber method. 


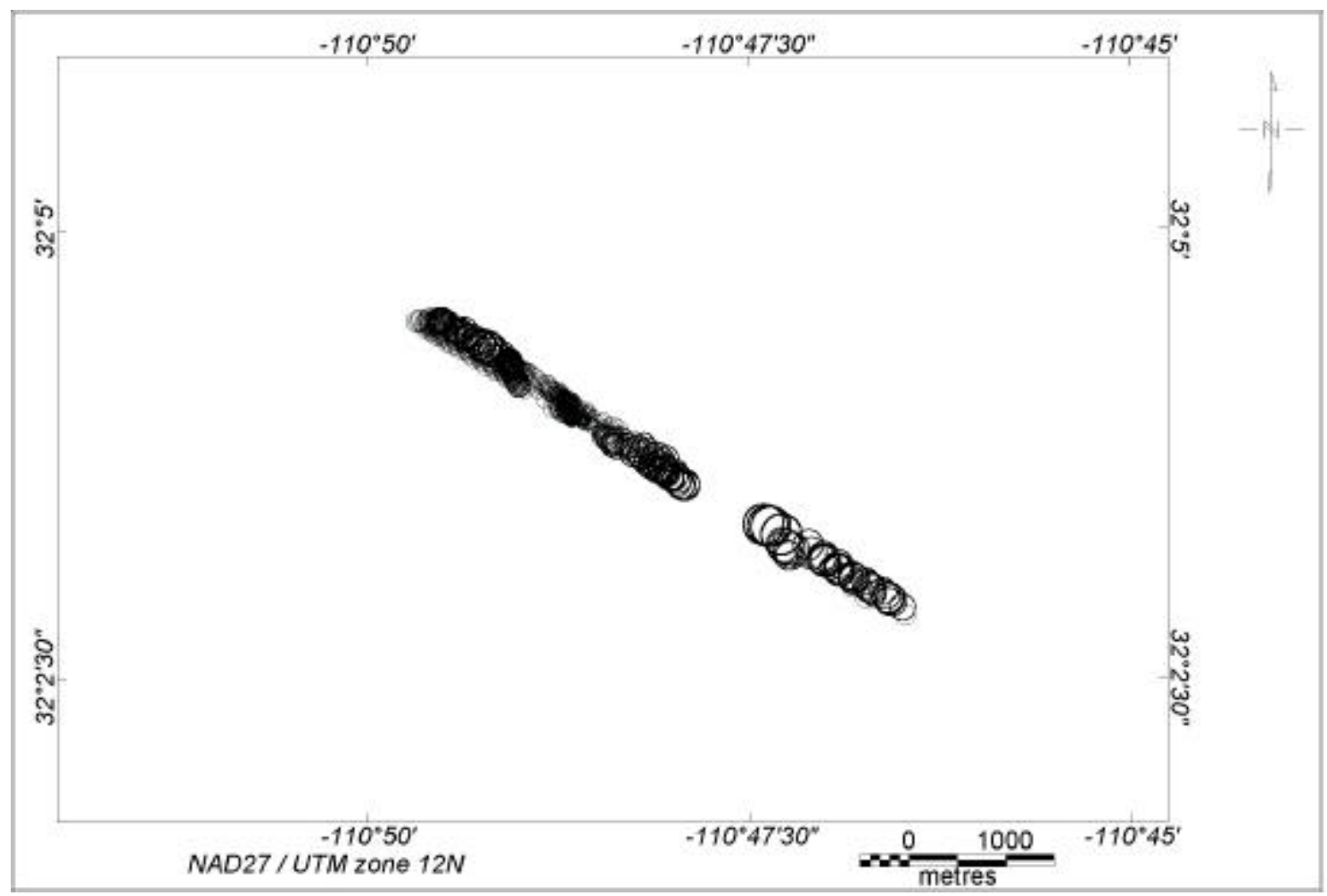

C7. Depth to source map over the pipelines using Euler deconvolution. 


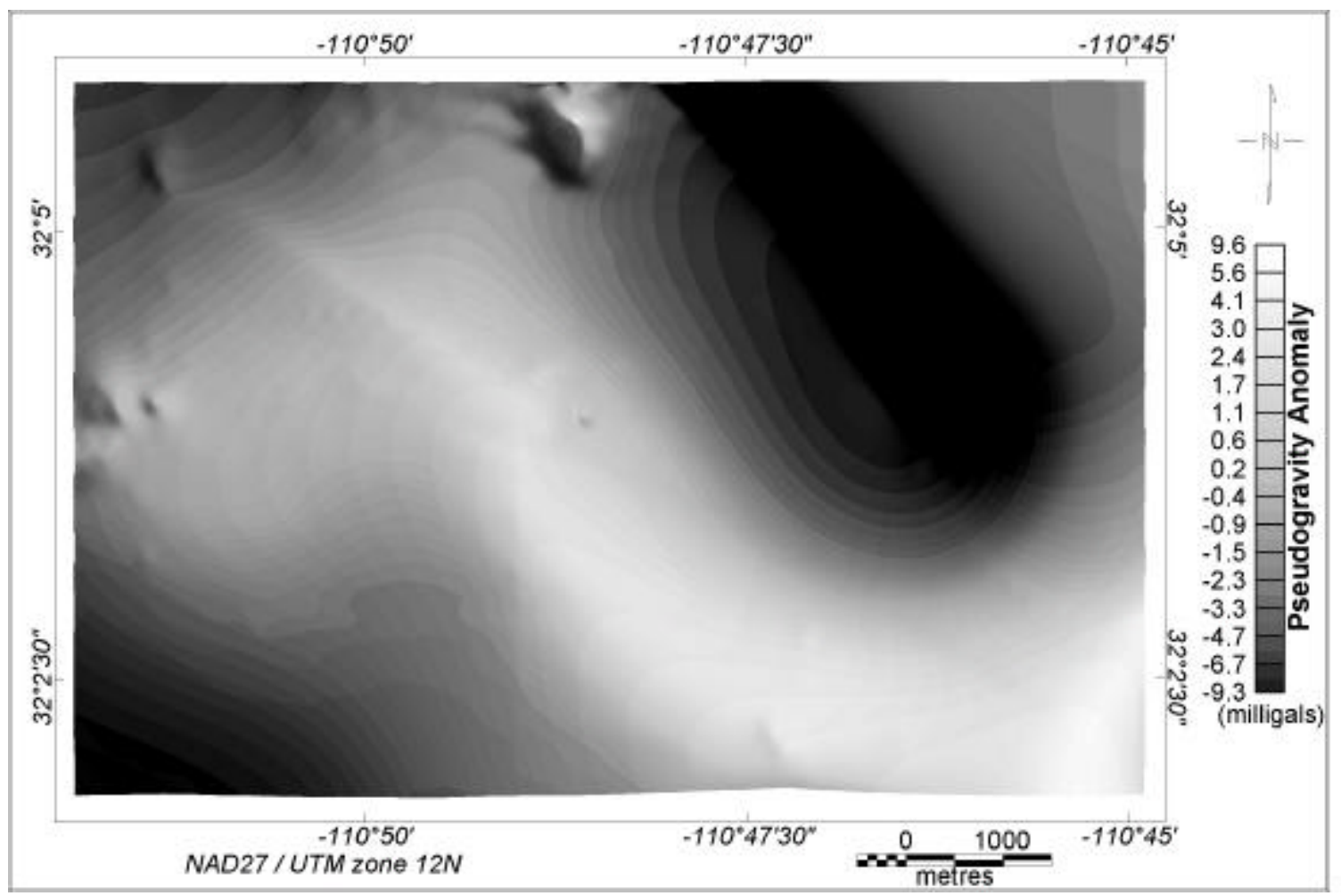

C8. Pseudo-gravity anomaly map over the natural gas pipelines. 


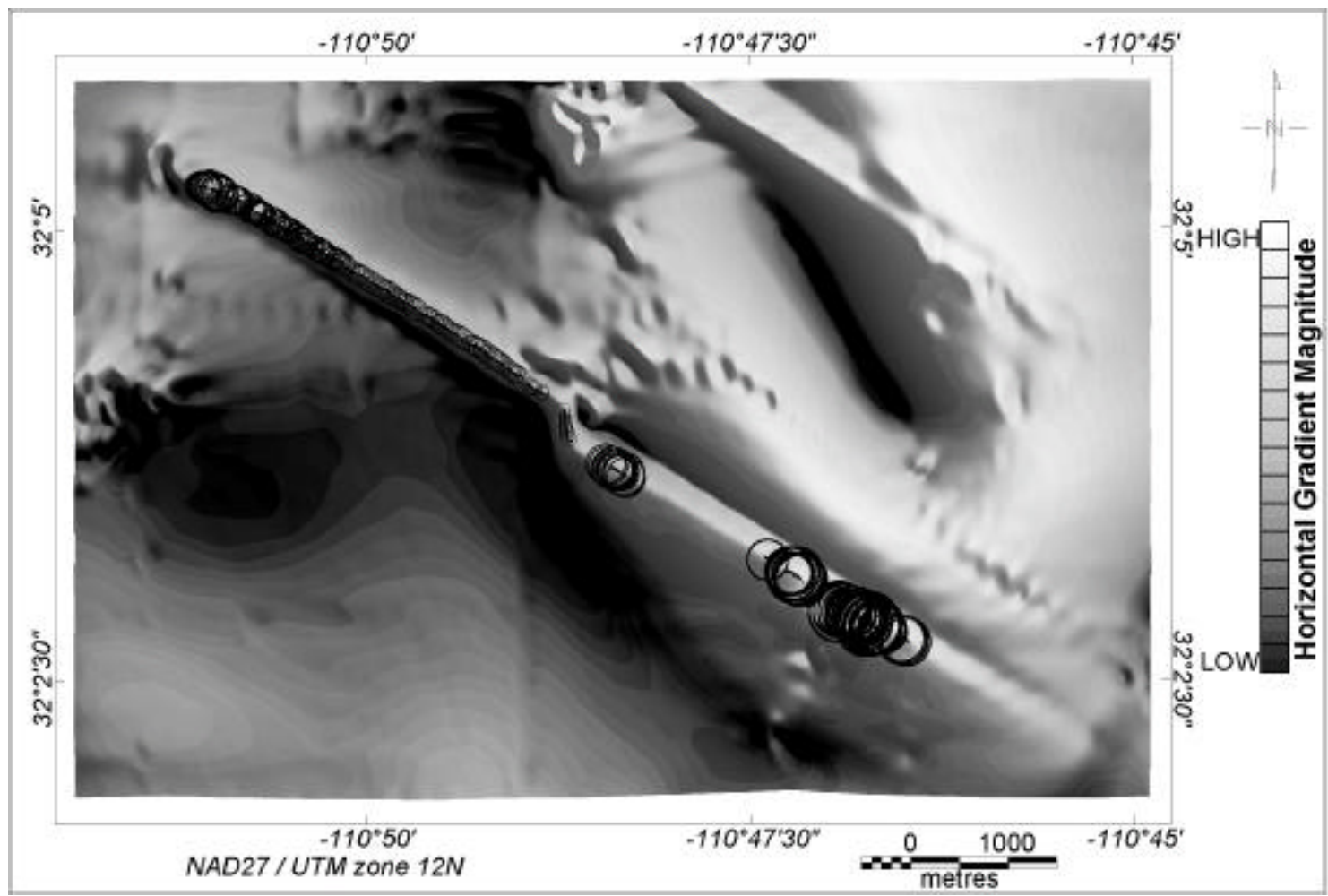

C9. Depth to source map over the pipelines using the horizontal gradient method applied to the pseudo-gravity field. 


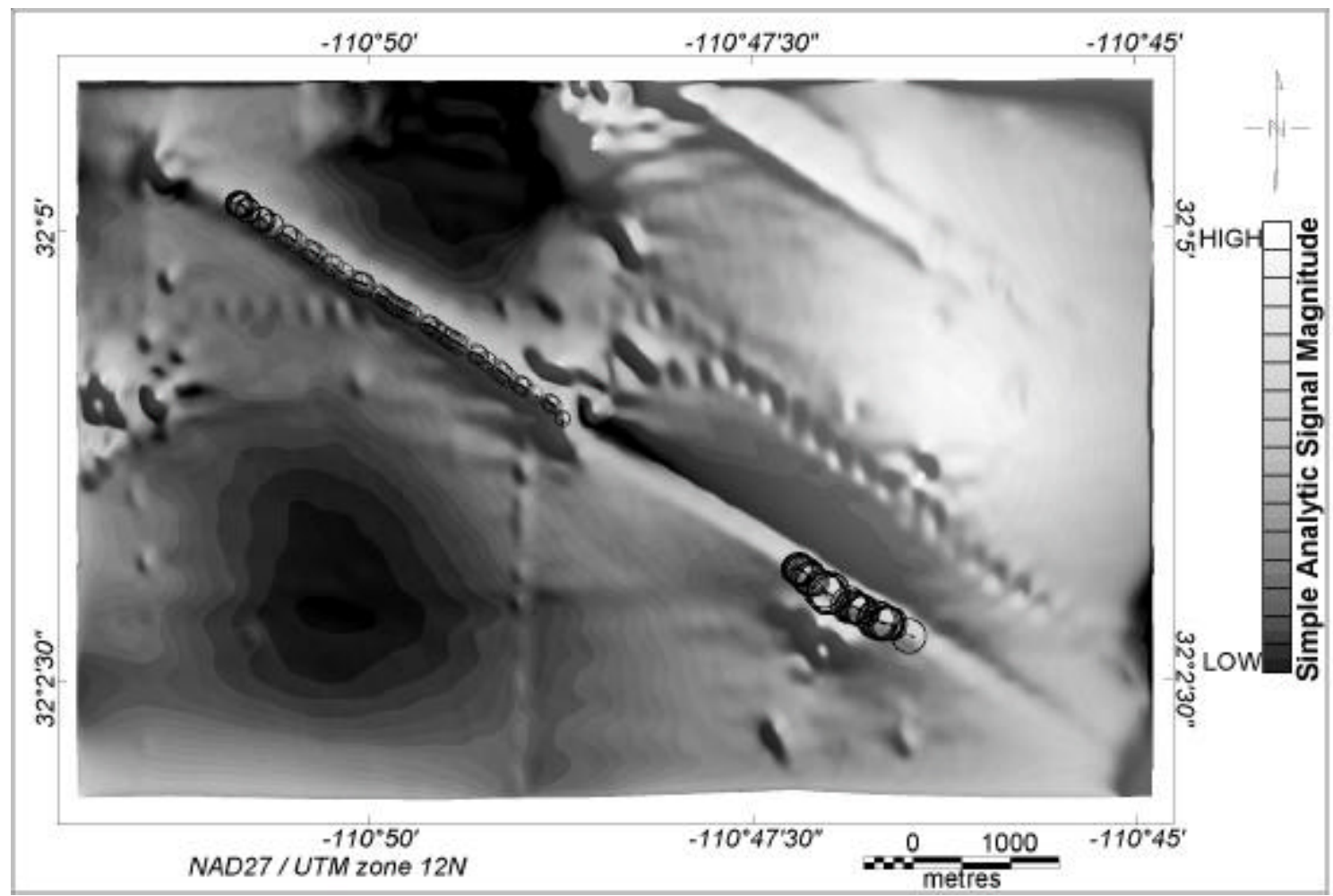

C10. Depth to source map over the pipelines using the simple analytic signal method applied to the pseudo-gravity field. 


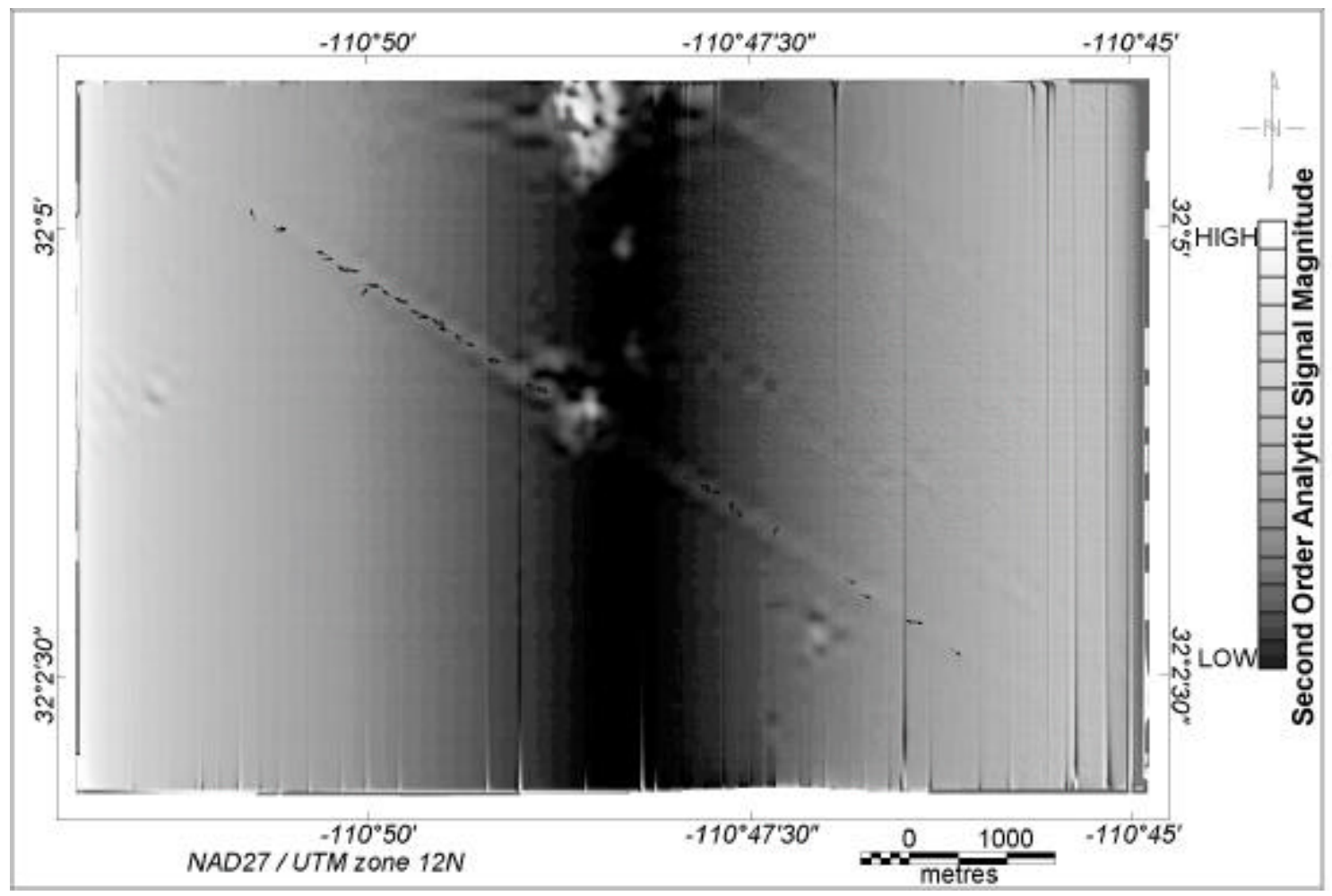

C11. Depth to source map over the pipelines using the enhanced analytic signal method (step model) applied to the pseudo-gravity field. 


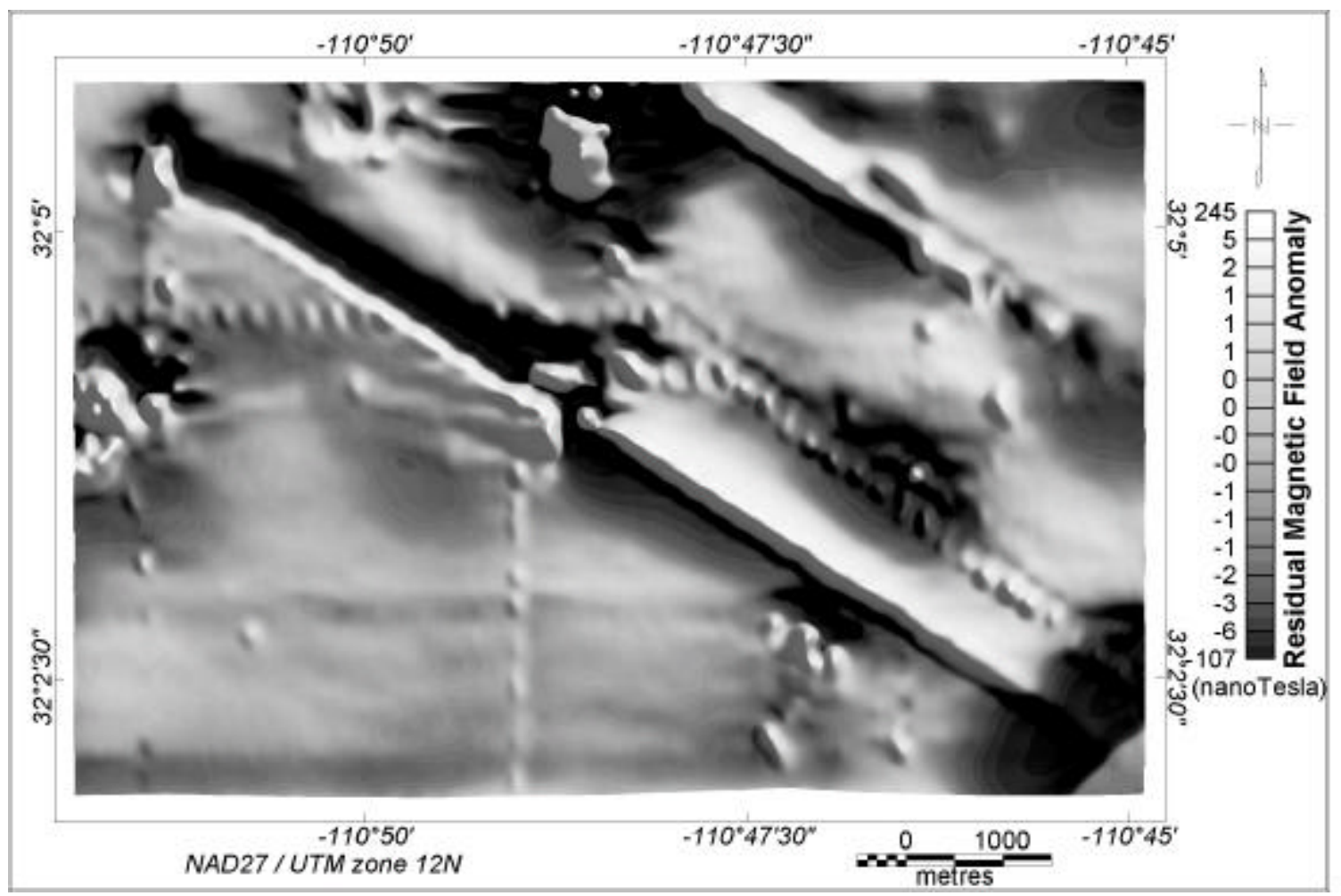

C12. High-pass filtered magnetic field anomaly map over the natural gas pipelines. 


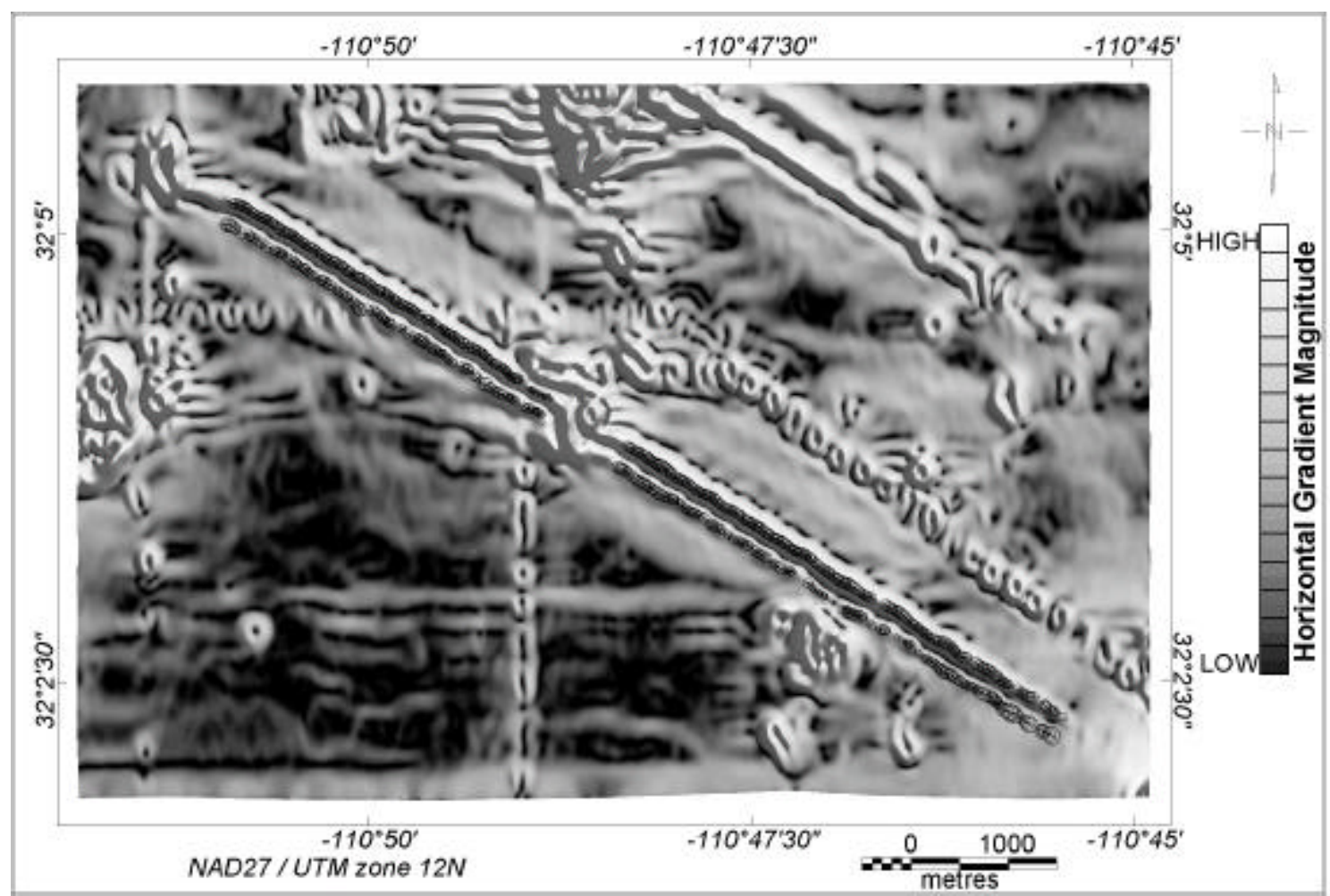

C13. Depth to source map over the pipelines using the horizontal gradient method applied to the high-pass filtered data. 


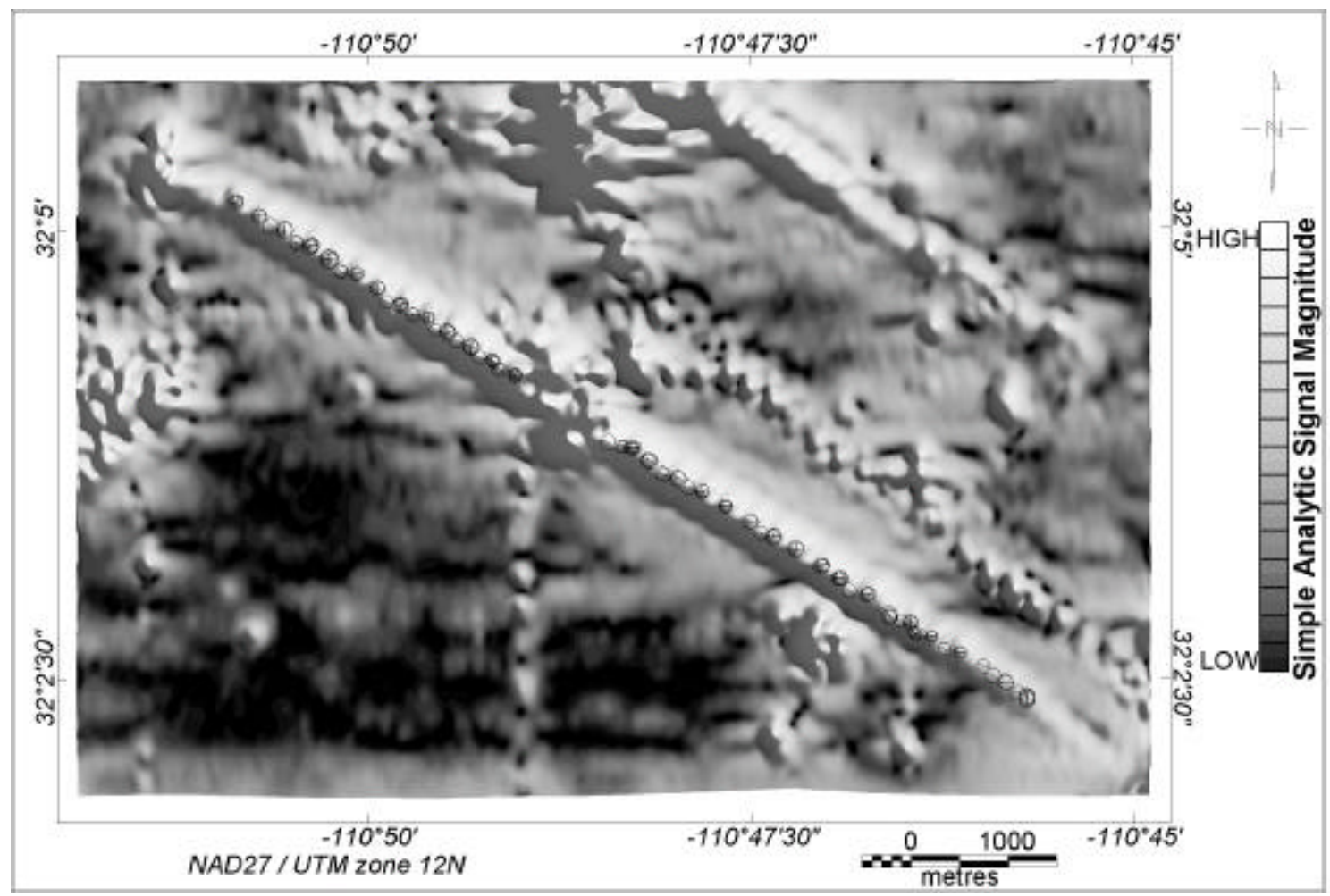

C14. Depth to source map over the pipelines using the simple analytic signal method applied to the high-pass filtered data. 


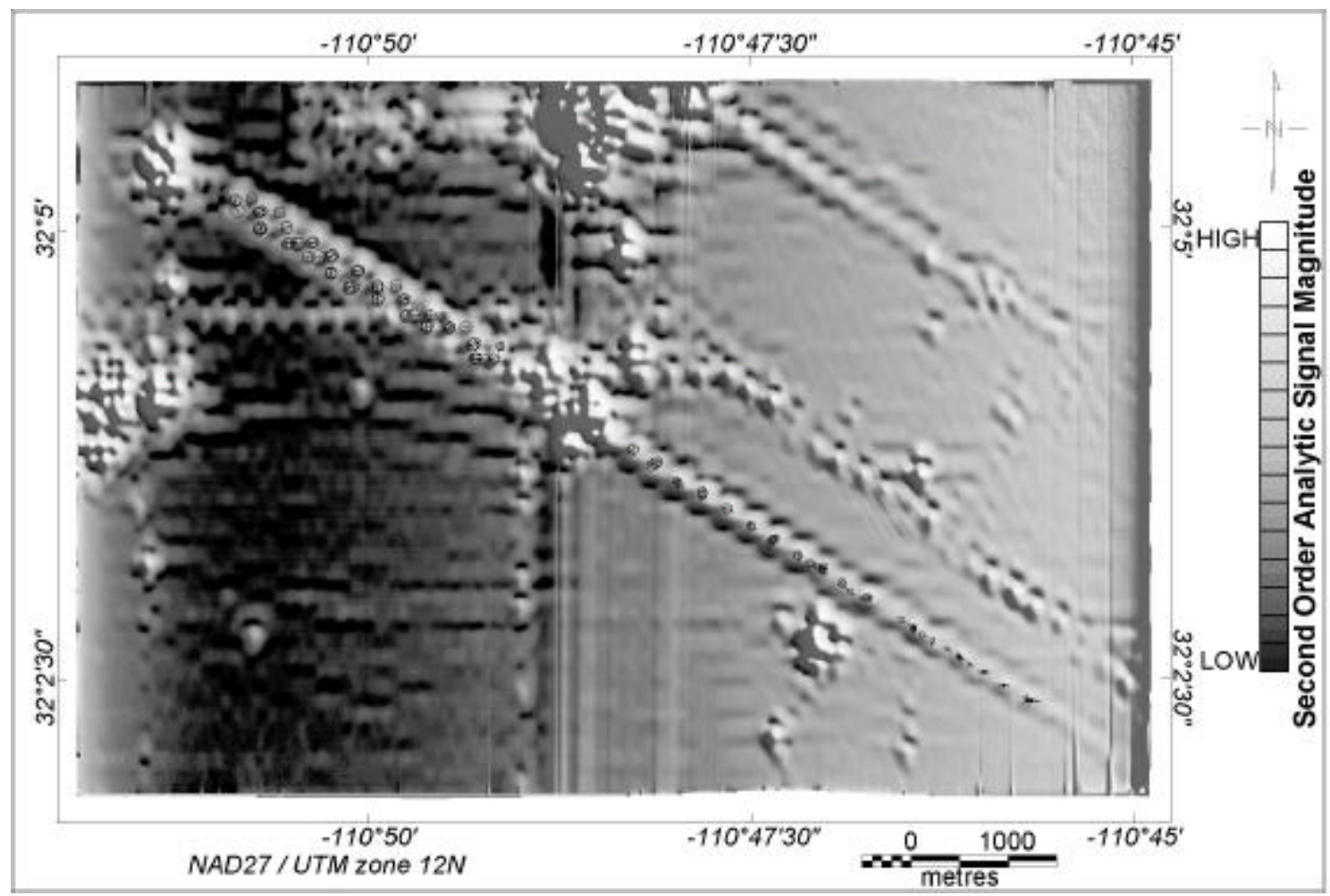

C15. Depth to source map over the pipe lines using the enhanced analytic signal method (step model) applied to the high-pass filtered data. 


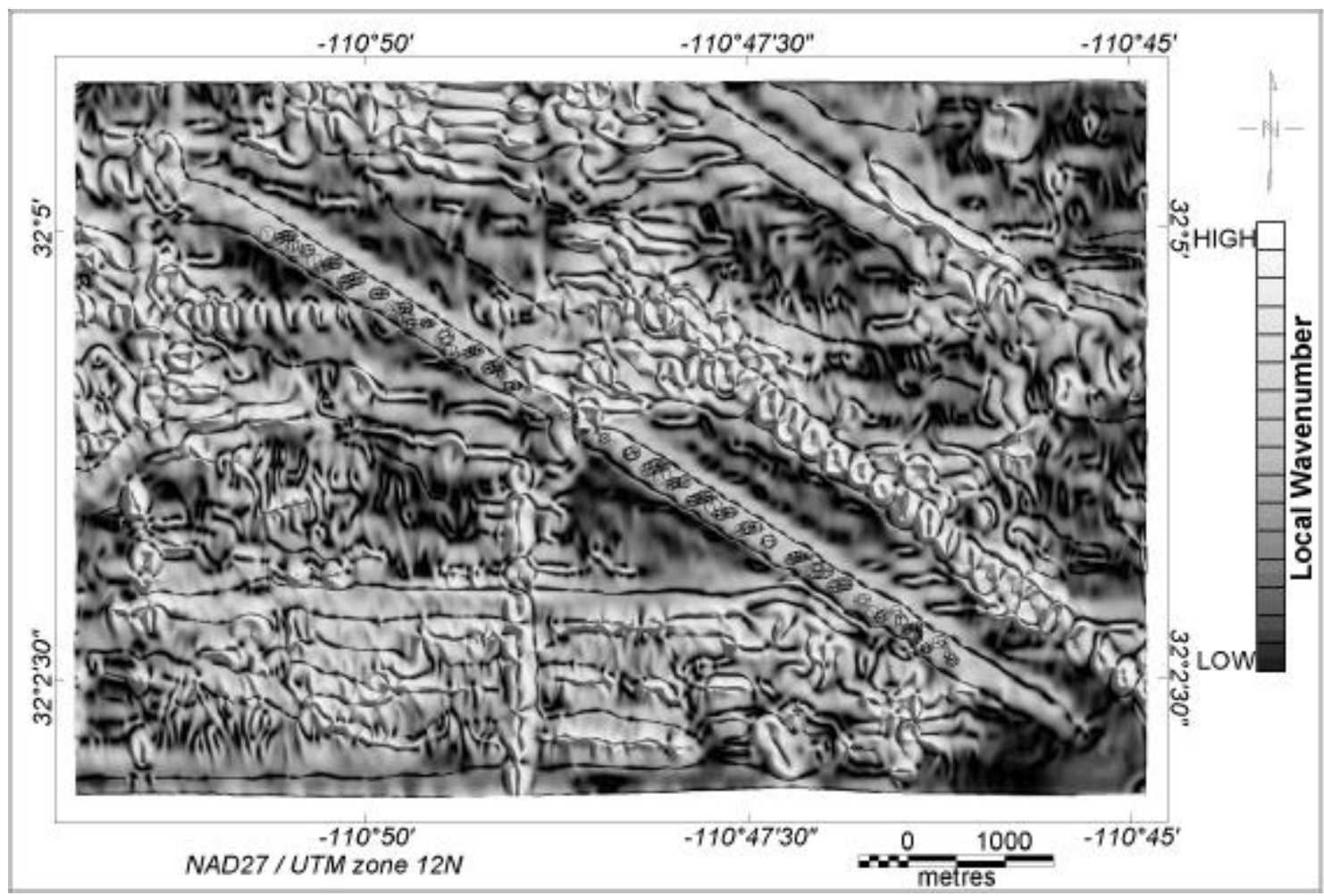

C16. Depth to source map over the pipelines using the local wavenumber method applied to the high-pass filtered data. 


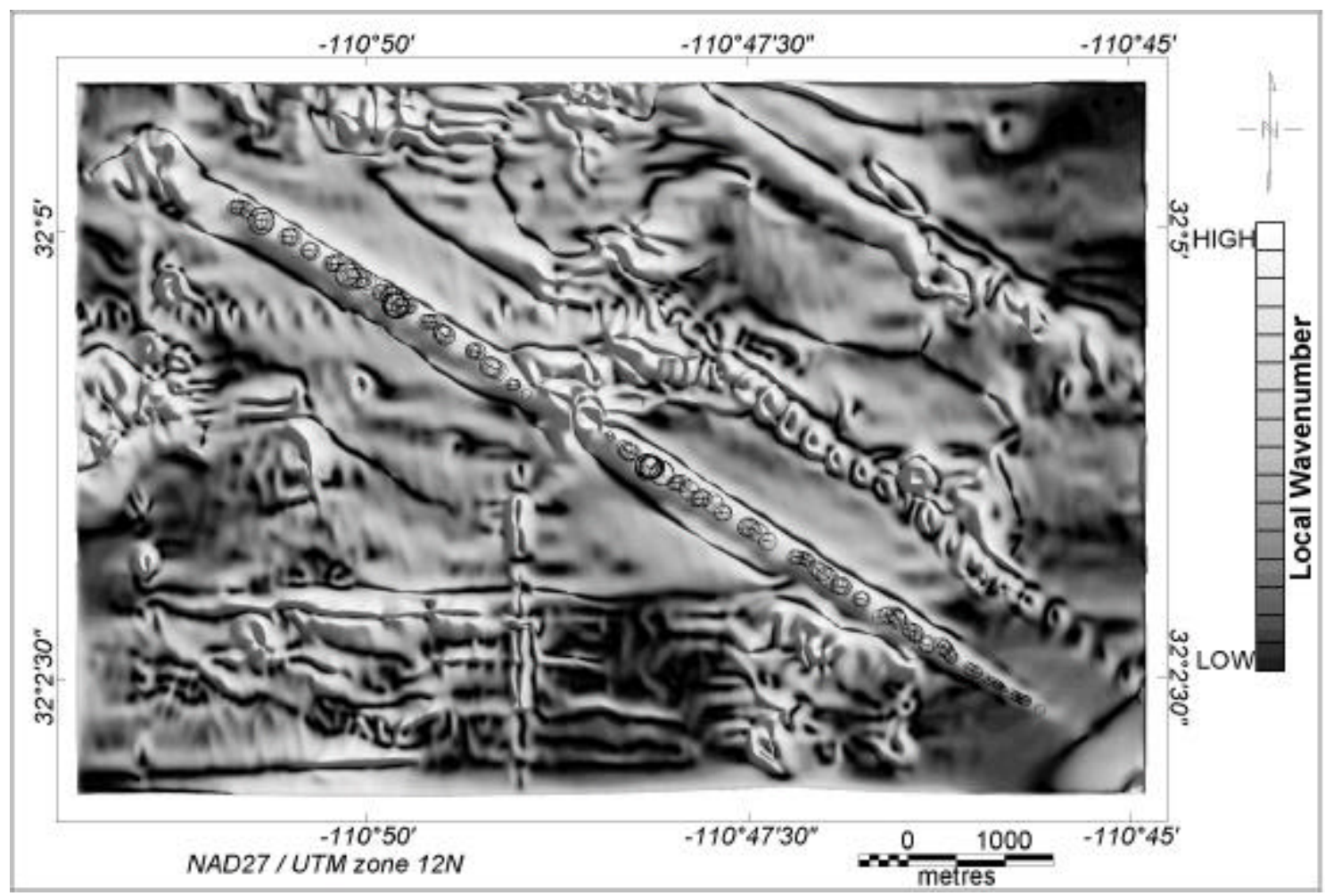

C17. Depth to source map over the pipelines using the local wavenumber method applied to the vertical integral of the residual magnetic field. 


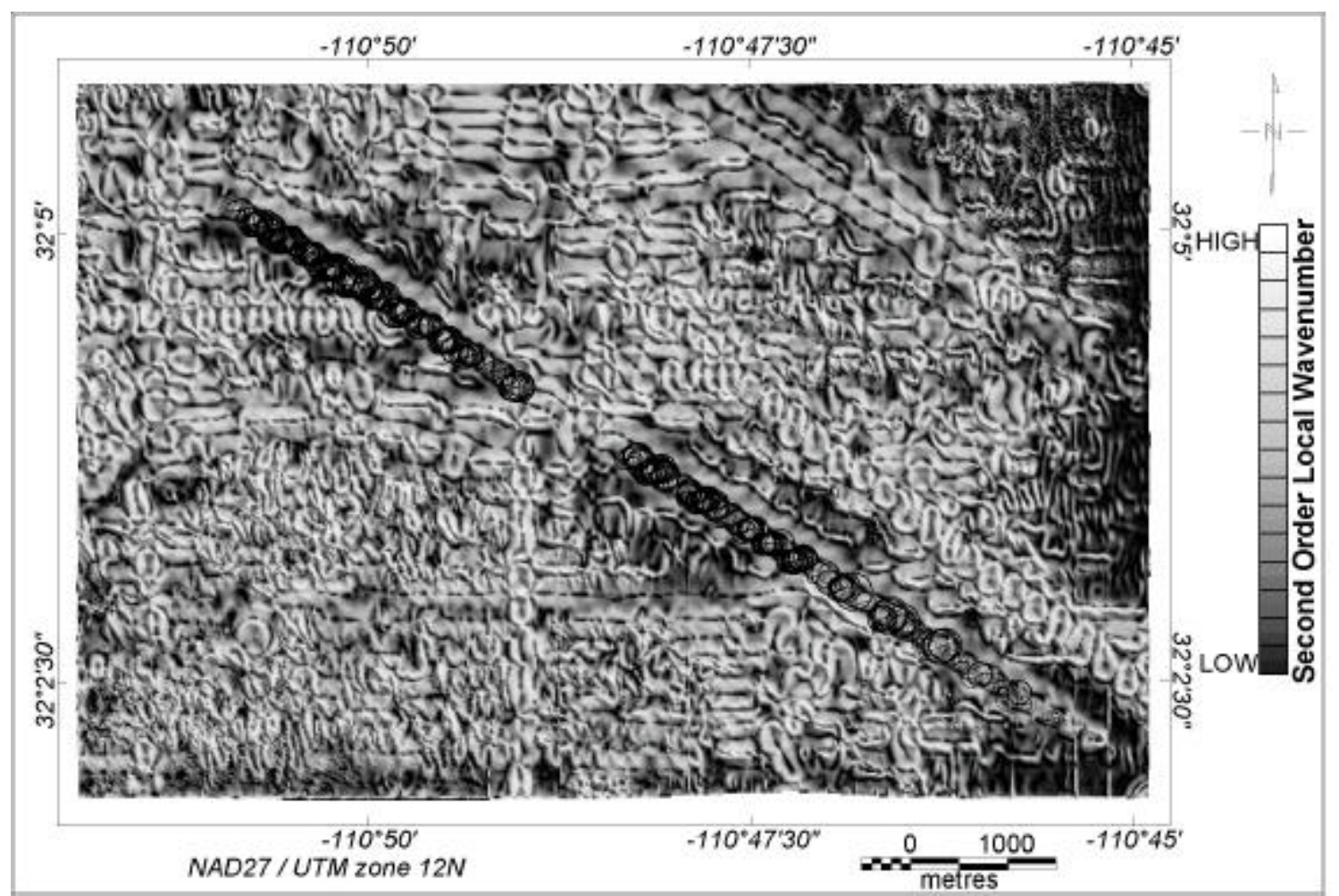

C18. Depth to source map over the pipelines using the enhanced local wavenumber method applied to the high-pass filtered data. 


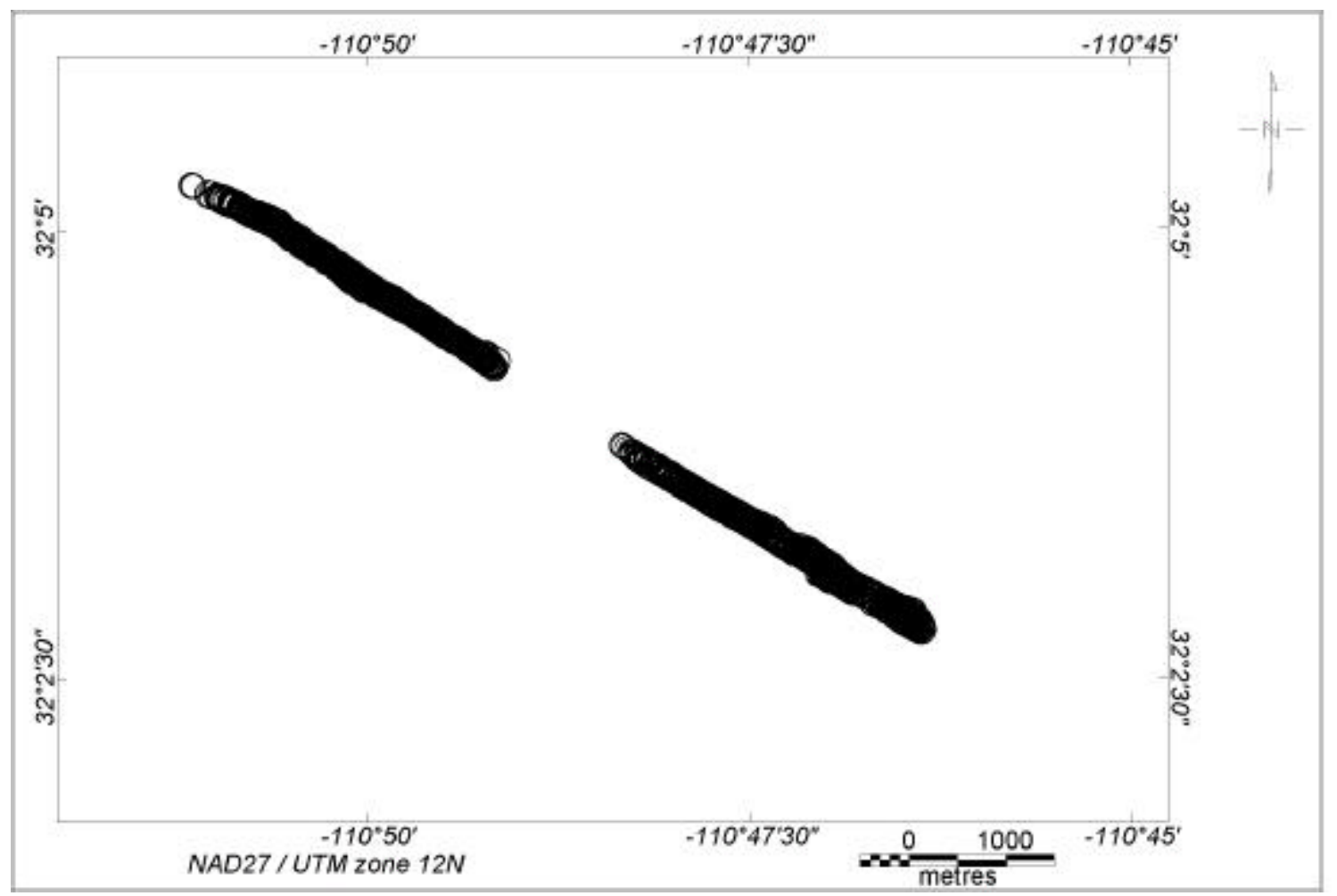

C19. Depth to source map over the pipelines using Euler deconvolution applied to the high-pass filtered data. 


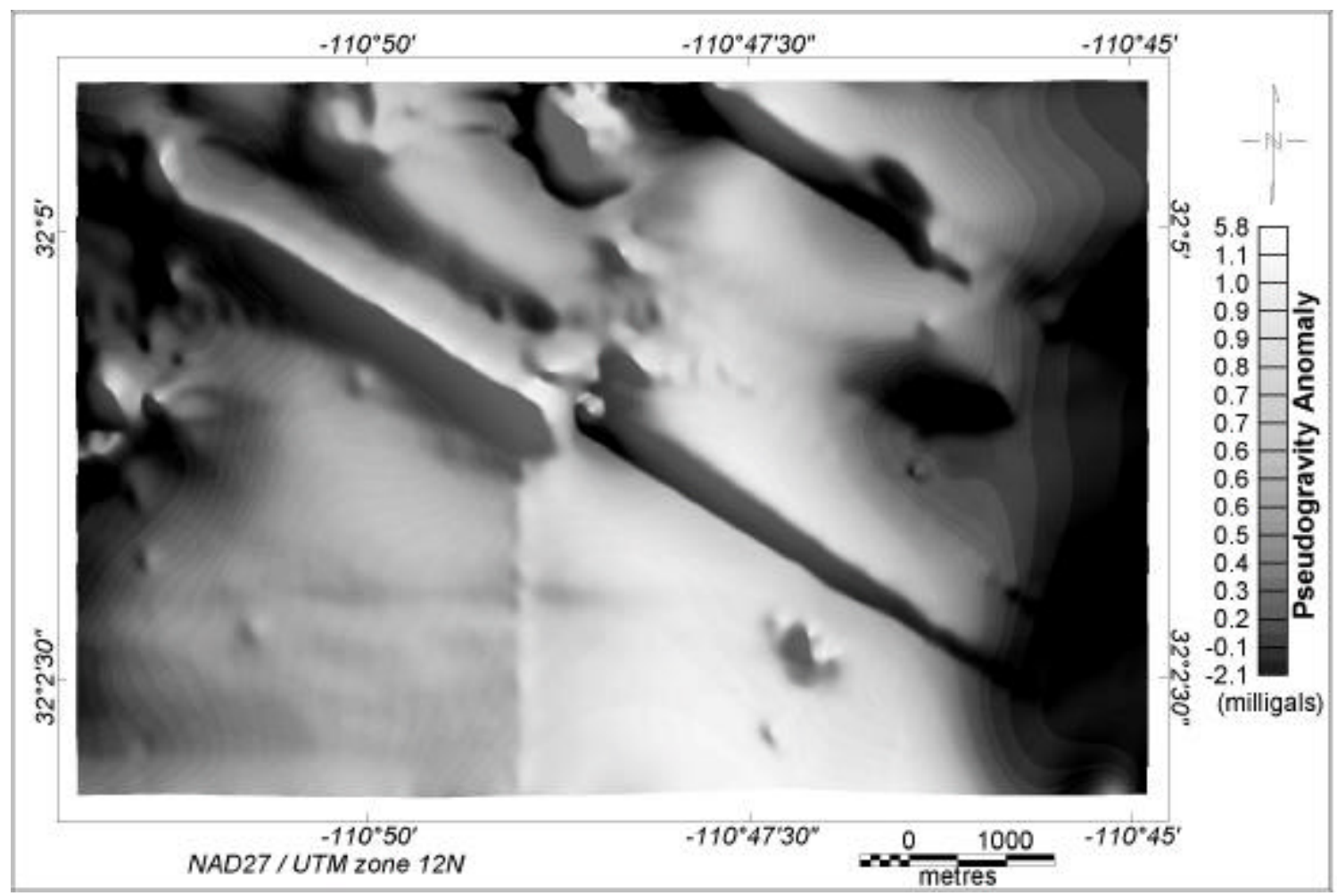

C20. High-pass filtered pseudo-gravity anomaly map over the natural gas pipelines. 


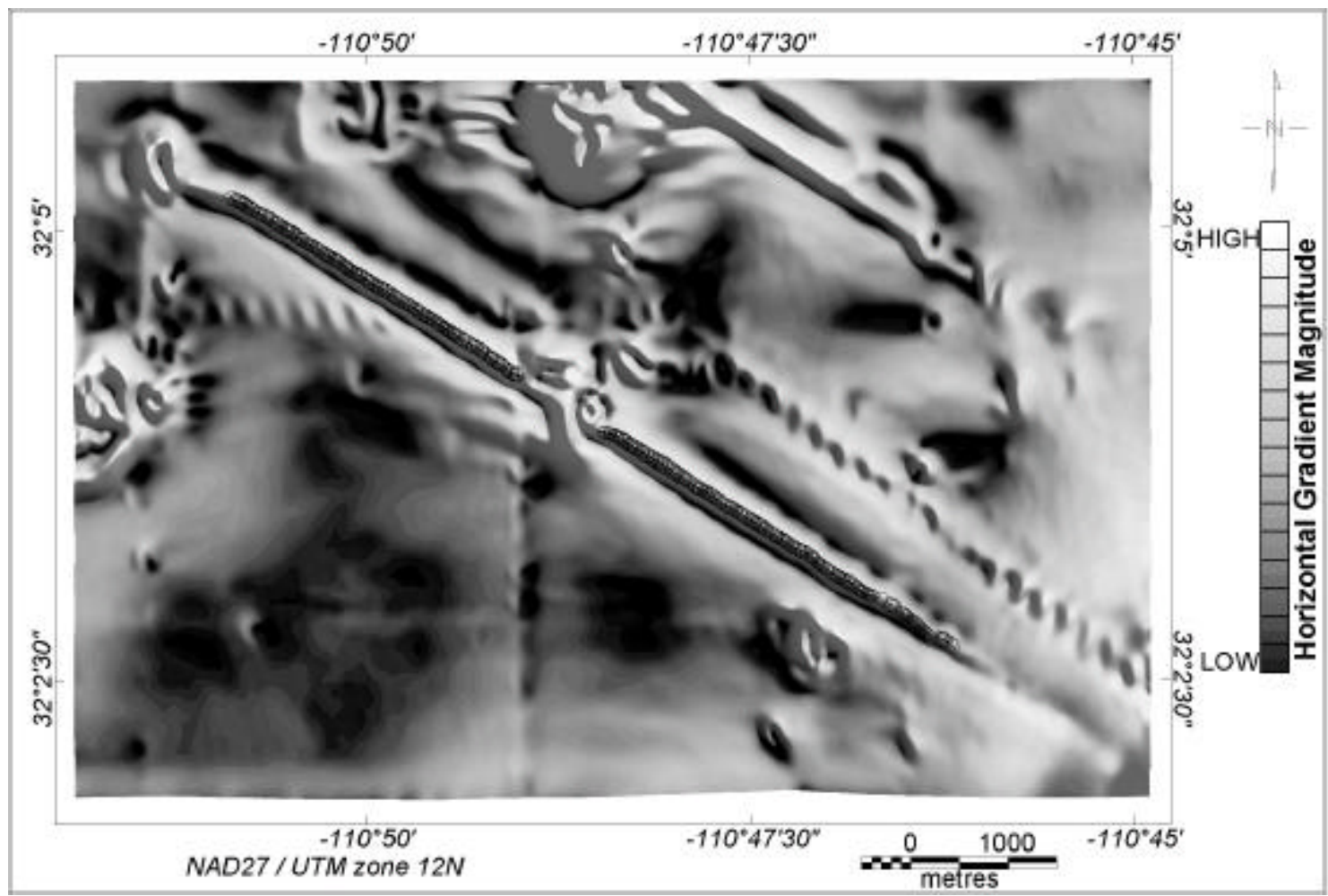

C21. Depth to source map over the pipelines using the horizontal gradient method applied to the high-pass filtered pseudo-gravity field. 


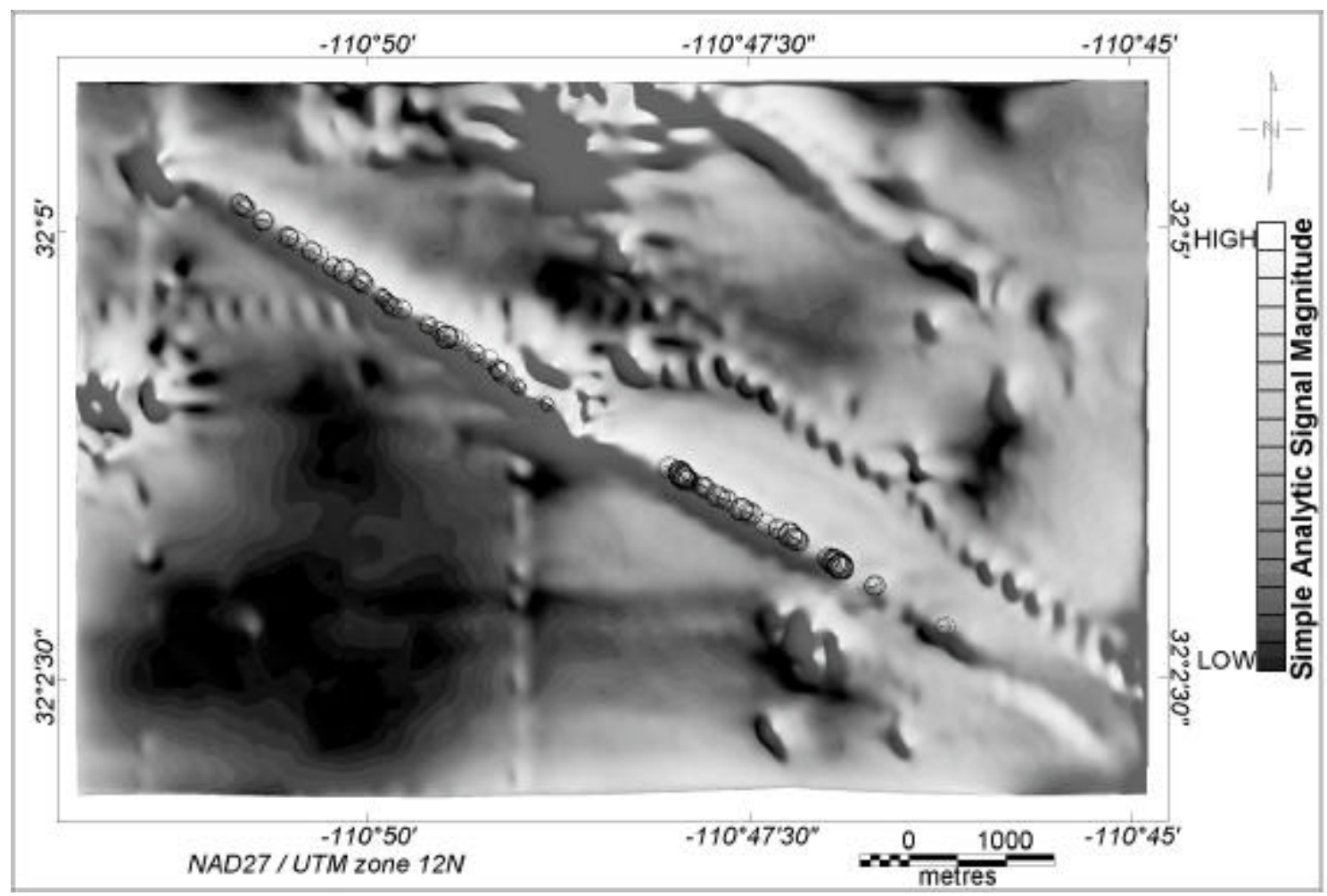

C22. Depth to source map over the pipelines using the simple analytic signal method applied to the high-pass filtered pseudo-gravity field. 


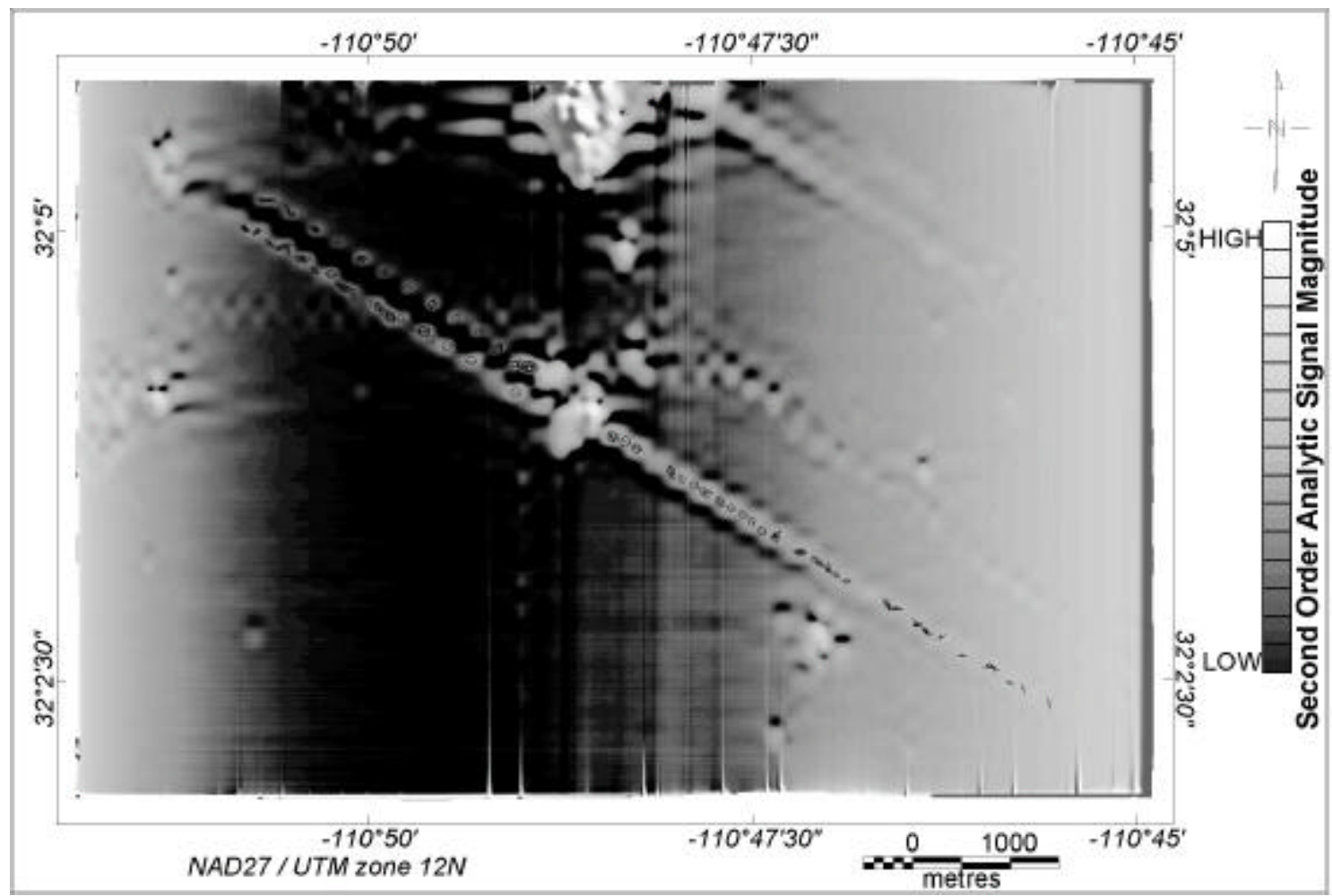

C23. Depth to source map over the pipelines using the enhanced analytic signal method (step model) applied to the high-pass filtered pseudo-gravity field. 


\section{REFERENCES}

Atchuta Rao, D., Ram Babu, H.V., and Sanker Narayan, P.V., 1981, Interpretation of magnetic anomalies due to dikes: The complex gradient method: Geophysics, Vol. 46, pp. 1572-1578.

Anderson, S.R., 1987, Cenozoic stratigraphy and geologic history of the Tucson basin, Pima County, Arizona: U.S. Geological Survey Water-Resources Investigations Report 87-4190, 20 p.

Baranov, V., 1957, A new method for interpretation of aeromagnetic maps: pseudogravimetric anomalies: Geophysics, Vol. 22, pp. 359-383.

Billings, S., and Richards, D., 2000, Quality control of gridded aeromagnetic data: Rio Tinto Exploration, Australia Region, 65p. Retrieved March, 02, 2001, from the World Wide Web: http://www.geosoft.com/.

Blakely, R.J., and Simpson, R.W., 1986, Approximating edges of source bodies from magnetic or gravity anomalies: Geophysics, Vol. 51, pp. 1494-1498.

Blatt, H., and Tracy, R.J., 1996, Petrology - Igneous, Sedimentary, and Metamorphic $2^{\text {nd }}$ ed.: W.H. Freeman and Company, 529p.

Burg, J.P., 1975, Maximum Entropy Spectral Analysis: Unpublished doctoral dissertation, Stanford University.

Cordell, L., and Grauch, V.J.S., 1985, Mapping basement magnetization zones from aeromagnetic data in the San Juan basin, New Mexico in Hinze, W.J., ed., The Utility of Regional Gravity and Magnetic Anomaly Maps: Society of Exploration Geophysicists, pp. 181-197.

Davidson, E.S., 1973, Geohydrology and water resources of the Tucson basin, Arizona: U.S. Geological Survey Water-Supply Paper 1939-E, 81p.

Desmons, B., 1998, High Sensitivity Aeromagnetic Survey, Tucson Basin, Arizona, USA: Sander Geophysics, Ltd., 26 p.

Doyle, F.J., 1997, Map conversion and the UTM grid: Photogrammetric Engineering and Remote Sensing, Vol. 63, no. 4, pp. 367-370

Drewes, H., 1980, Tectonic Map of Southeast Arizona: U.S. Geological Survey Miscellaneous Investigations Map I-1109, scale 1:25000. 
Fraser, D.C, Fuller, B.D., and Ward, S.H., 1966, Some numerical techniques for application in mining exploration: Geophysics, Vol. 31, pp. 1066-1077.

Geosoft, Inc., 1998, OASIS montaj, Data Processing and Analysis (DPA) System, version 4.3: Geosoft, Inc.

Grauch, V.J.S., and Cordell, L., 1987, Short note: Limitations of determining density or magnetic boundaries from the horizontal gradient of gravity or pseudogravity data: Geophysics, Vol. 52, pp. 118-121.

Halliday, D., Resnick, R., and Walker, J., 1993, Fundamentals of Physics $-4^{\text {th }}$ ed.: John Wiley \& Sons, Inc., 1130p.

Houser, B.B., and Gettings, M.E., 2000, Stratigraphy and tectonic history of the Tucson basin, Arizona, based on re-examination of cuttings and geophysical logs of the Exxon State (32)-1 well - a preliminary report: U.S. Geological Survey Open-File Report 00-139, 38 p.

Hsu, S.-K., Coppens, D., and Shyu, C.-T., 1998, Depth to magnetic source using the generalized analytic signal: Geophysics, Vol. 63, pp. 1947-1957.

Hsu, S.-K, Sibuet, J.-C., and Shyu, C.,-T, 1996, High-resolution detection of geologic boundaries from potential-field anomalies: An enhanced analytic signal technique: Geophysics, Vol. 61, pp. 373-386.

Kearey, P., and Brooks, M., 1991, An Introduction to Geophysical Exploration - $2^{\text {nd }}$ ed.: Blackwell Scientific Publications, 254 p.

Macomber, M.M., 1984, World geodetic system, 1984: US Defense Mapping Agency, $11 \mathrm{p}$.

Nabighian, M.N., 1972, The analytic signal of two-dimensional magnetic bodies with polygonal cross-section: its properties and use for automated anomaly interpretation: Geophysics, Vol. 37, pp. 507-517.

Nabighian, M.N., 1984, Toward a three-dimensional automatic interpretation of potential field data via generalized Hilbert transforms: Fundamental relations: Geophysics, Vol. 49, pp. 780-786.

Phillips, J.D., 1997, Potential-field geophysical software for the PC, version 2.2: U.S. Geological Survey Open-File Report 97-725, 34 p. (online at ftp://greenwood.cr.usgs.gov/pub/open-file-reports/ofr-97-0725/pfofr.htm). 
Phillips, J.D., 2001, Processing and interpretation of aeromagnetic data for the Santa Cruz basin - Patagonia Mountains area, south-central Arizona - a preliminary report: U.S. Geological Survey Open-File Report 15 p.

Reid, J.M., Allsop, J.M., Granser, A.J., and Somerton, I.W., 1990, Magnetic interpretation in three dimensions using Euler deconvolution: Geophysics, Vol. 55 , pp. 80-91.

Roest, W.R., and Pilkington, M., 1993, Identifying remanent magnetization effects in magnetic data: Geophysics, Vol. 58, pp. 653-659.

Roest, W.R., Verhoef, J., and Pilkington, M., 1992, Magnetic interpretation using the 3-D analytic signal: Geophysics, Vol. 57, pp. 116-125.

Smith, R.S., Thurston, J.B., Dai, T.-F., and MacLeod, I.N., 1998, iSPI ${ }^{\mathrm{TM}}$ - the improved source parameter imaging method: Geophysical Prospecting, pp. 141-151.

Smith, W.H.F., and Wessel, P., 1990, Gridding with continuous curvature splines in tension: Geophysics, Vol. 55, pp. 293-305.

Telford, W.M., Geldart, L.P., Sheriff, R.E., 1990, Applied Geophysics - $2^{\text {nd }}$ ed.: Cambridge University Press, 770 p.

Thompson, D.T., 1982, EULDPH: A new technique for making computer-assisted depth estimates from magnetic data: Geophysics, Vol. 47, pp. 31-37.

Thurston, J.B., and Smith, R.S., 1997, Automatic conversion of magnetic data to depth, dip, and susceptibility contrast using the SPI TM method: Geophysics, Vol. 62, pp. 807-813.

TopoZone, 2000, Maps a la carte, Inc. Retrieved March, 15, 2001, from the World Wide Web: http://www.topozone.com/. 